This is a self-archived version of an original article. This version may differ from the original in pagination and typographic details. Author(s): $\begin{aligned} & \text { Blaum, K.; Eliseev, S.; Danevich, F. A.; Tretyak, V. I.; Kovalenko, Sergey; } \\ & \text { Krivoruchenko, M. I.; Novikov, Yu. N.; Suhonen, J. }\end{aligned}$

Title: Neutrinoless double-electron capture

Year: 2020

Version: Published version

Copyright: @ 2020 American Physical Society

Rights: In Copyright

Rights url: http://rightsstatements.org/page/lnC/1.0/?language=en

Please cite the original version:

Blaum, K., Eliseev, S., Danevich, F. A., Tretyak, V. I., Kovalenko, S., Krivoruchenko, M. I., Novikov, Yu. N., \& Suhonen, J. (2020). Neutrinoless double-electron capture. Reviews of Modern Physics, 92(4), Article 045007. https://doi.org/10.1103/RevModPhys.92.045007 
REVIEWS OF MODERN PHYSICS, VOLUME 92, OCTOBER-DECEMBER 2020

\title{
Neutrinoless double-electron capture
}

\author{
K. Blaum $\odot$ and S. Eliseev \\ Max-Planck-Institut für Kernphysik, Saupfercheckweg 1, 69117 Heidelberg, Germany \\ F. A. Danevich $\odot$ and V. I. Tretyak $\odot$ \\ Institute for Nuclear Research of NASU, Prospekt Nauky 47, Kyiv 03028, Ukraine
}

Sergey Kovalenko

Departamento de Ciencias Físicas, Universidad Andres Bello, Sazié 2212, Santiago 8320000, Chile

\author{
M. I. Krivoruchenko \\ Institute for Theoretical and Experimental Physics, NRC "Kurchatov Institute", \\ B. Cheremushkinskaya 25, 117218 Moscow, Russia \\ and National Research Centre "Kurchatov Institute", Ploschad' Akademika Kurchatova 1, \\ 123182 Moscow, Russia
}

\author{
Yu. N. Novikov \\ Petersburg Nuclear Physics Institute, NRC "Kurchatov Institute", \\ Gatchina, 188300 St. Petersburg, Russia \\ and Department of Physics, St. Petersburg State University, 199034 St. Petersburg, Russia
}

\section{J. Suhonen}

Department of Physics, University of Jyvaskyla, P.O. Box 35, Jyvaskyla Fl-40014, Finland

\section{(published 16 December 2020)}

\begin{abstract}
Double-beta processes play a key role in the exploration of neutrino and weak interaction properties, and in the searches for effects beyond the standard model. During the last half century many attempts were undertaken to search for double-beta decay with emission of two electrons, especially for its neutrinoless mode $0 \nu 2 \beta^{-}$, the latter having still not been observed. Double-electron capture (2EC) was not yet in focus because of its in general lower transition probability. However, the rate of neutrinoless double-electron capture $0 \nu 2 \mathrm{EC}$ can experience a resonance enhancement by many orders of magnitude when the initial and final states are energetically degenerate. In the resonant case, the sensitivity of the $0 \nu 2 \mathrm{EC}$ process can approach the sensitivity of the $0 \nu 2 \beta^{-}$decay in the search for the Majorana mass of neutrinos, right-handed currents, and other new physics. An overview of the main experimental and theoretical results obtained during the last decade in this field is presented. The experimental part outlines search results of 2EC processes and measurements of the decay energies for possible resonant $0 \nu 2 \mathrm{EC}$ transitions. An unprecedented precision in the determination of decay energies with Penning traps has allowed one to refine the values of the degeneracy parameter for all previously known near-resonant decays and has reduced the rather large uncertainties in the estimate of the $0 \nu 2 \mathrm{EC}$ half-lives. The theoretical part contains an updated analysis of the electron shell effects and an overview of the nuclear-structure models, in which the nuclear matrix elements of the $0 \nu 2 \mathrm{EC}$ decays are calculated. One can conclude that the decay probability of $0 \nu 2 \mathrm{EC}$ can experience a significant enhancement in several nuclides.
\end{abstract}

DOI: 10.1103/RevModPhys.92.045007

\section{CONTENTS}

I. Introduction

II. Double-Electron Capture and Physics beyond the Standard Model

A. Quark-level mechanisms of $0 \nu 2 \mathrm{EC}$

B. Examples of underlying high-scale models

C. Hadronization of quark-level interactions
III. Phenomenology of Neutrinoless 2EC

A. Underlying formalism

B. Decay amplitude of the light Majorana neutrino exchange mechanism

C. Comparison of $0 \nu 2 \mathrm{EC}$ and $0 \nu 2 \beta^{-}$decay half-lives 12

IV. Electron Shell Effects

A. Interaction energy of electron holes 15

1. Electrostatic interaction 
2. Retardation correction in the Feynman gauge

3. Magnetostatic interaction in the Feynman gauge

4. Magnetostatic interaction in the Coulomb gauge

5. Gauge invariance of the interaction energy of electron holes

B. Double-electron ionization potentials in Auger spectroscopy

C. Summary

V. Nuclear Matrix Elements

A. Overview of the calculated nuclear matrix

elements in $0 \nu 2 \mathrm{EC}$

B. Overview of the calculation frameworks

1. Multiple-commutator model

2. Deformed quasiparticle random-phase approximation

3. Microscopic interacting boson model

4. Energy-density functional method

C. Decays of nuclides with the calculated nuclear matrix elements

D. Summary

VI. Status of Experimental Searches

A. Experimental studies of 2EC processes

B. Prospects for possible future experiments

C. Neutrinoless 2EC with radioactive nuclides

D. Summary

VII. Precise Determination of 2EC Decay Energies

A. Basics of high-precision Penning-trap mass spectrometry

B. Decay energies of 2EC transitions in virtually stable nuclides

C. Prospects for measurements of decay energies in radioactive nuclides

VIII. Normalized Half-Lives of Near-Resonant Nuclides

A. Decays of virtually stable nuclides

B. Decays of long-lived radionuclides

IX. Conclusions

List of Symbols and Abbreviations

Acknowledgments

References

\section{INTRODUCTION}

In 1937, Ettore Majorana found an evolution equation for a truly neutral spin-1/2 fermion (Majorana, 1937). His work was motivated by the experimental observation of a free neutron by James Chadwick in 1932. Majorana also conjectured that his equation applies to a hypothetical neutrino introduced by Wofgang Pauli to explain the continuous spectrum of electrons in the $\beta$ decay of nuclei. In the mid1950s, Reines and Cowan $(1956,1959)$ discovered a particle with neutrino properties. At the time of the establishment of the standard model (SM) of electroweak interactions, three families of neutrinos were known. While the neutron is a composite fermion consisting of quarks, neutrinos acquired the status of elementary particles with zero masses in the SM framework. The discovery of neutrino oscillations by the Super-Kamiokande Collaboration (Fukuda et al., 1998) showed that neutrinos are mixed and massive. A comprehensive description of the current state of neutrino physics was given by Bilenky (2018).

Whether neutrinos are truly neutral fermions is one of the fundamental questions in modern particle physics, astrophysics, and cosmology. The fermions described by the Dirac equation can also be electrically neutral. However, even in this case there is a conserved current in Diracs theory, which ensures a constant number of particles (minus antiparticles). Majorana fermions, like photons, do not have such a conserved current. In Dirac's theory, particles and antiparticles are independent, while Majorana fermions are their own antiparticles and, via $C P T$, it follows that they must have zero charge. Truly neutral spin- $1 / 2$ fermions are referred to as Majorana fermions, and those with a conserved current are referred to as Dirac fermions. Majorana fermions in bispinor basis are vectors of the real vector space $\mathbb{R}^{4}$. They can also be described by two-component Weyl spinors in the complex space $\mathbb{C}^{2}$. In both representations, the superposition principle for Majorana fermions holds over the field of real numbers. Majorana fermions belong to the fundamental real representation of the Poincaré group. A Dirac fermion of mass $m$ can be represented as a superposition of two Majorana fermions of masses $m$ and $-m$, respectively.

The neutron has a nonvanishing magnetic moment, so it cannot be a pure Majorana particle. On the other hand, there is no fundamental reason to claim that it is a pure Dirac particle. In theories with nonconservation of the baryon number, the mass eigenstates include a mixture of baryons and antibaryons. At the phenomenological level, the effect is modeled by adding a Majoranian mass term to the effective Lagrangian. As a result, the neutron experiences oscillations $n \leftrightarrow \bar{n}$ whereby the nuclei decay with nonconservation of the baryon number (Dover, Gal, and Richard, 1983, 1985; Krivoruchenko, 1996a, 1996b; Gal, 2000; Kopeliovich and Potashnikova, 2010; Phillips et al., 2016). Experimental limits for the period of the $n \leftrightarrow \bar{n}$ oscillations in the vacuum $\tau_{\text {vac }}>2.7 \times 10^{8} \mathrm{~s}$ (Abe et al., 2015) constrain the neutron Majorana mass to $\Delta m \sim 1 / \tau_{\mathrm{vac}}<0.8 \times 10^{-33} \mathrm{~m}$, where $m=939.57 \mathrm{MeV} / c^{2}$ is the neutron Dirac mass. Thus, under the condition of nonconservation of the baryon number, Majorana's idea on the existence of truly neutral fermions can be partially realized in relation to the neutron. In contrast, neutrinos can be pure Majorana fermions or pure Dirac fermions or a mixture of these two extreme cases. It is noteworthy that none of the variants of neutrino masses are possible within the SM. The neutrino mass problem leads us to physics beyond the SM. Alternative examples of Majorana particles include weakly interacting dark-matter candidates and Majorana zero modes in solid state systems (Elliott and Franz, 2015).

Searches for neutrinoless double-beta $\left(0 \nu 2 \beta^{-}\right)$decay, neutrinoless double-electron capture $(0 \nu 2 \mathrm{EC})$ by nuclei, and other lepton number violating (LNV) processes provide the possibility to shed light on the question of the nature of neutrinos whether they are Majorana or Dirac particles. By virtue of the black-box theorem (Schechter and Valle, 1982; Hirsch, Kovalenko, and Schmidt, 2006), observation of the $0 \nu 2 \beta$ decay would prove that neutrinos have a finite Majorana mass. The massive Majorana neutrinos lead to a violation of the conservation of the total lepton number $L$. In the quark sector of the SM, the baryon charge $B$ is a similar quantum number. Vector currents of $B$ and $L$ are classically conserved. Lefthanded fermions are coupled to the $\mathrm{SU}(2)$ electroweak gauge fields $W^{ \pm}$and $Z^{0}$ so that vector currents of $B$ and $L$, 


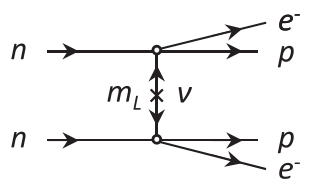

FIG. 1. Schematic representation of neutrinoless double-beta decay. Two neutrons in the nucleus experience $\beta$ decay accompanied by the exchange of a Majorana neutrino. Neutrons, protons, electrons, and neutrinos are represented by solid lines. Arrows show the flow of baryon charge of protons and neutrons and the flow of lepton charge of electrons and neutrinos. The cross denotes the Majorana neutrino mass term $m_{L}$ that causes the helicity flip of the intermediate neutrino and violates the lepton number by two units.

as 't Hooft (1976) first pointed out, are sensitive to the axial anomaly. Through electroweak instantons, this leads to nonconservation of $B$ and $L$, while the difference $B-L$ is conserved. The violating amplitude is exponentially suppressed. Another example is given by sphaleron solutions of classical field equations of the SM that preserve $B+L$ but violate $B$ and $L$ individually, which can be relevant for cosmological implications (White, 2016). The conservation of $B-L$ within the SM is not supported by any fundamental principles analogous to local gauge symmetry, so $B$ and $L$ can be broken beyond the SM explicitly. Experimental observation of the proton decay or $n \leftrightarrow \bar{n}$ oscillations could prove nonconservation of $B$, while observation of the $0 \nu 2 \beta^{-}$decay or the neutrinoless double-electron capture $(0 \nu 2 \mathrm{EC})$ could prove nonconservation of $L$ with constant $B$. Moreover, these processes are of interest for determining the absolute neutrino mass scale, the type of neutrino mass hierarchy, and the character of $C P$ violation in the lepton sector. Because of the exceptional value of LNV processes, a vast literature is devoted to physics of $0 \nu 2 \beta^{-}$decay and the underlined nuclear-structure models; see Bilenky and Petcov (1987), Suhonen (2007), Avignone, Elliott, and Engel (2008), Vergados, Ejiri, and Šimkovic (2012), Raduta (2015), Engel and Menxéndez (2017), and Ejiri, Suhonen, and Zuber (2019).

The $0 \nu 2 \beta^{-}$decay was first discussed by Furry (1939). The process is shown in Fig. 1. A nucleus with the mass number $A$ and charge $Z$ experiences $0 \nu 2 \beta^{-}$decay accompanied by the exchange of a Majorana neutrino between the nucleons

$$
(A, Z) \rightarrow(A, Z+2)^{++}+e^{-}+e^{-},
$$

where $(A, Z+2)^{++}$is the doubly ionized atom in the final state. There are many models beyond the SM that provide alternative mechanisms of the $0 \nu 2 \beta^{-}$decay, some of which are discussed in Sec. II.

In 1955 , the related $0 \nu 2 \mathrm{EC}$ process

$$
e_{b}^{-}+e_{b}^{-}+(A, Z) \rightarrow(A, Z-2)^{* *}
$$

was discussed by Winter (1955a). Here $e_{b}^{-}$are bound electrons. The nucleus and the electron shell of the neutral atom $(A, Z-2)^{* *}$ are in excited states. An example of the

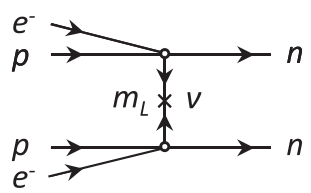

FIG. 2. Schematic representation of neutrinoless doubleelectron capture. Two protons in the nucleus each capture a bound electron from the electron shell and turn into two neutrons by the exchange of a Majorana neutrino. Notation is the same as in Fig. 1.

mechanism related to the Majorana neutrino exchange is shown in Fig. 2. Subsequent deexcitation of the nucleus occurs via gamma-ray radiation or $\beta$ decays. Deexcitation of the electron shell is associated with the emission of Auger electrons or gamma rays in a cascade formed by the filling of electron vacancies. Absent special selection rules, dipole radiation dominates in $\mathrm{x}$ rays. Since the dipole moment of electrons is much higher than that of nucleons in the nucleus, the deexcitation of the electron shell goes faster. For atoms with a low value of $Z$, the Auger-electron emission is more likely. With an increase in the atomic number, the radiation of x-ray photons becomes dominant. The deexcitation of high electron orbits is due to Auger-electron emission for all $Z$.

Estimates show that the sensitivity of the $0 \nu 2 \mathrm{EC}$ process to the Majorana neutrino mass is many orders of magnitude lower than that of the $0 \nu 2 \beta^{-}$decay. Winter pointed out that degeneracy of the energies of the parent atom $(A, Z)$ and the daughter atom $(A, Z-2)^{* *}$ gives rise to resonant enhancement of the decay. In the early 1980s, Georgi, Glashow, and Nussinov (1981) and Voloshin, Mitsel'makher, and Éramzhyan (1982) also remarked on the possible resonant enhancement of the $0 \nu 2 \mathrm{EC}$ process. The resonances in $2 \mathrm{EC}$ were considered, however, an unlikely coincidence.

To compensate for the low probability of the $0 \nu 2 \mathrm{EC}$ process by a resonance effect, it is necessary to determine the energy difference of atoms with high accuracy. The decay probability is proportional to the Breit-Wigner factor $\Gamma_{f} /\left(\Delta^{2}+\Gamma_{f}^{2} / 4\right)$, where $\Gamma_{f}$ is the electromagnetic decay width of the daughter atom and $\Delta=M_{A, Z}-M_{A, Z-2}^{* *}$ is the degeneracy parameter equal to the mass difference of the parent and the daughter atoms. The maximum increase in probability is achieved for $\Delta=0$ when the decay amplitude approaches the unitary limit. Taking $\Delta \sim 10 \mathrm{keV}$ for the typical splitting of the masses of the atoms and $\Gamma_{f} \sim 10 \mathrm{eV}$ for the typical decay width of the excited electron shell of the daughter atom, one finds a maximum enhancement of $\sim 10^{6}$. The degeneracy parameter $\Delta \lesssim \Gamma_{f}$ gives the half-life of a nuclide with respect to $0 \nu 2 \mathrm{EC}$ comparable to the half-life of nuclides with respect to $0 \nu 2 \beta^{-}$decay.

The near-resonant $0 \nu 2 \mathrm{EC}$ process was analyzed in detail by Bernabeu, De Rujula, and Jarlskog (1983). They developed a nonrelativistic formalism of the resonant $0 \nu 2 \mathrm{EC}$ in atoms and specified a dozen of nuclide pairs for which degeneracy is not excluded. The $0 \nu 2 \mathrm{EC}$ process became the subject of a detailed theoretical study by Sujkowski and Wycech (2004). A list of the near-resonant $0 \nu 2 \mathrm{EC}$ nuclide pairs was also provided by 
Karpeshin (2008). The problem acquired an experimental character: the difference between masses of the parent and daughter atoms, i.e., $Q$ values, known to an accuracy of about $10 \mathrm{keV}$, which is too far from the accuracy required to identify the unitary limit. The determination of the degeneracy parameter has acquired fundamental importance.

In the 1980s, there was no well-developed technique to measure the masses of nuclides with relative uncertainty of about $10^{-9}$ sufficient to find resonantly enhanced $0 \nu 2 \mathrm{EC}$ processes. The current state-of-the-art technique highprecision Penning-trap mass spectrometry was still in its infancy. Its triumphal advance in the field of high-precision mass measurements on radioactive nuclides began with the installation of the ISOLTRAP facility at CERN in the late 1980s (Bollen et al., 1987; Mukherjee et al., 2008; Kluge, 2013). Recent decades have been marked by a rising variety of high-precision Penning-trap facilities in Europe, the USA, and Canada (Blaum, 2006; Blaum, Dilling, and Nörtershäuser, 2013). This led to a tremendous development of Penning-trap mass-measurement techniques (Eliseev et al., 2007, 2013, 2014; George, Blaum et al., 2007; Kretzschmar, 2007, 2013; Blaum, Dilling, and Nörtershäuser, 2013) and made it possible to routinely carry out mass measurements on a broad variety of nuclides with a relative uncertainty of about $10^{-9}$. The mass of the ion is determined via the measurement of its free cyclotron frequency in a pure magnetic field, the most precisely measurable quantity in physics.

These factors motivated a new study of the near-resonant $0 \nu 2 \mathrm{EC}$ process. A relativistic formalism for calculating electron shell effects was developed and an updated realistic list of nuclide pairs for which the measurement of $Q_{2 \mathrm{EC}}$ values has high priority was compiled (Krivoruchenko et al., 2011). A significantly refreshed database of the nuclides and their excited states is now available, 30 years after the previous publication by Bernabeu, De Rujula, and Jarlskog (1983). An overview of the investigation of the resonant $0 \nu 2 \mathrm{EC}$ was given by Eliseev, Novikov, and Blaum (2012) including persistent experimental attempts to search for appropriate candidates for this extraordinary phenomenon.

The advancements of the experiments in search of the $0 \nu 2 \mathrm{EC}$ process are more modest than those searching for $0 \nu 2 \beta^{-}$decay. While the sensitivity of the $0 \nu 2 \beta^{-}$experiments approaches half-life limits $T_{1 / 2} \sim 10^{24}-10^{26} \mathrm{yr}$, which constrains the effective Majorana neutrino mass of electron neutrino to $\left|m_{\beta \beta}\right| \lesssim 0.1-0.7 \mathrm{eV}$, the results of the best $0 \nu 2 \mathrm{EC}$ experiments are still approximately $T_{1 / 2} \sim 10^{19}-10^{22}$ yr. The reasons for this difference are rather obvious: there is usually a much lower relative abundance of the isotopes of interest (typically lower than $1 \%$ ), and additionally a more complicated effect signature due to the emission of a gamma-quanta cascade (instead of a clear $0 \nu 2 \beta^{-}$peak at the decay energy). The second circumstance results in a lower detection efficiency for the most energetic peak in a $0 \nu 2 \mathrm{EC}$ energy spectrum. Furthermore, the energy of the most energetic $0 \nu 2 \mathrm{EC}$ peak is generally lower than in the $0 \nu 2 \beta^{-}$processes, yet the higher the energy of a certain process the better the suppression of the radioactive background. As a result the scale of the 2EC experiments is substantially smaller than that of the
$0 \nu 2 \beta^{-}$ones. At the same time, there is a motivation to search for the neutrinoless $\mathrm{EC} \beta^{+}$and $2 \beta^{+}$decays owing to the potential to clarify the possible contribution of the righthanded currents to the $0 \nu 2 \beta^{-}$decay rate (Hirsch et al., 1994) and the appealing possibility of the resonant $0 \nu 2 \mathrm{EC}$ processes. The complicated effect signature expected in resonant $0 \nu 2 \mathrm{EC}$ transitions becomes an advantage: the detection of several gamma quanta with well-known energies could be a strong proof of the pursued effect.

The previously mentioned aspects of the phase space, degeneracy, abundance factors, etc., play an important role in determining the half-lives of the $0 \nu \mathrm{EC} \beta^{+}$and $0 \nu 2 \mathrm{EC}$ processes. A further ingredient affecting the decay half-lives are the involved nuclear matrix elements (NMEs); see Suhonen (2012a), Maalampi and Suhonen (2013), and Ejiri, Suhonen, and Zuber (2019). These NMEs have been calculated in various nuclear-theory frameworks for a number of nuclei. In this review we use these NMEs, as well as NMEs that have been calculated just for this review, to estimate the half-lives of those $0 \nu 2 \mathrm{EC}$ transitions that are of interest due to their possibly favorable resonance conditions.

\section{DOUBLE-ELECTRON CAPTURE AND PHYSICS BEYOND THE STANDARD MODEL}

The underlying quark-level physics behind the $0 \nu 2 \mathrm{EC}$ process [see Eq. (1.2)] is basically the same as for the $0 \nu 2 \beta^{-}, 0 \nu 2 \beta^{+}$, and $0 \nu \mathrm{EC} \beta^{+}$decays. In Figs. 1 and 2 , we show the mechanism of exchange of light or heavy neutrinos with Majorana mass. The latter arise beyond the SM within the Weinberg dimension-5 effective LNV operator (Weinberg, 1979) providing conditions for the existence of the processes $0 \nu 2 \mathrm{EC}$ and $0 \nu 2 \beta^{-}$. A violation of the lepton number can also occur from quark-lepton effective Lagrangians of higher dimensions, corresponding to other possible mechanisms of the $0 \nu 2 \mathrm{EC}$ process. The neutrinoless $2 \mathrm{EC}$ can be accompanied by the emission of one or more extremely light particles other than neutrinos in $2 \nu 2 \mathrm{EC}$. A well-known example is the Majoron $J$ as the Goldstone boson of a spontaneously broken $\mathrm{U}(1)_{L}$ symmetry of the lepton number. Passing to the hadronic level one meets two possibilities of hadronization of the quark-level underlying process known from $0 \nu 2 \beta^{-}$decay: direct nucleon and pionic mechanisms. Next, we will take a closer look at the previously mentioned aspects of the $0 \nu 2 \mathrm{EC}$ process.

First, the underlying quark-level mechanisms of the neutrinoless 2EC can be classified according to the following possible exotic final states:

- No exotic particles in the final state. The reaction $0 \nu 2 \mathrm{EC}$ is shown in Eq. (1.2).

- The reaction $0 \nu 2 \mathrm{EC} n J: e_{b}^{-}+e_{b}^{-}+(A, Z) \rightarrow(A, Z-2)^{* *}+$ $n J$, with $n$ being the number of Majorons or Majoronlike exotic particles in the final state.

Both kinds of reactions can be further classified by the typical distance between particles involved in the underlying quark-lepton process, depending on the masses of the intermediate particles (Päs et al., 1999, 2001; Prezeau, Ramsey-Musolf, and Vogel, 2003; Cirigliano et al., 2017a, 2018a), as illustrated in Fig. 3. 


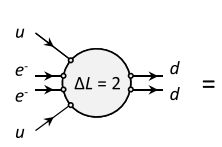

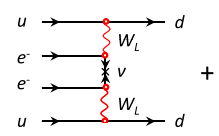

(a)

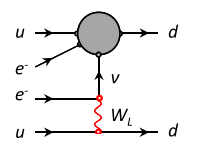

(b)

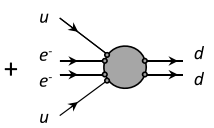

(c)
FIG. 3. A decomposition of the generic $\Delta L=2$ vertex into (a), (b) the long-range and (c) short-range quark-level contributions to $0 \nu 2$ EC. (a) The conventional Majorana neutrino mass mechanism. The blobs in (b) and (c) denote the effective $\Delta L=2$ vertices.

- Long-range mechanisms with the Weinberg $d=5$ operator providing Majorana mass to the intermediate neutrino in Fig. 3(a) and an effective $d=6$ operator in the upper vertex of Fig. 3(b).

- Short-range mechanisms with a dimension-9 effective operator in the vertex of Fig. 3(c).

The effective operators in the low-energy limit originate from diagrams with heavy exotic particles in the internal lines.

The diagrams of the $0 \nu 2 \mathrm{EC} n J$ decays are derived from those in Fig. 3 by inserting one or more scalar Majoron lines either into the blobs of effective operators or into the central neutrino line of Fig. 3(a).

\section{A. Quark-level mechanisms of $0 \nu 2 \mathrm{EC}$}

We now consider in more detail the short- and long-range mechanisms of $0 \nu 2 \mathrm{EC}$. The corresponding diagrams for the 0 $\nu 2$ EC process are shown in Fig. 3. The blobs in Figs. 3(b) and 3(c) represent the $\Delta L=2$ effective vertices beyond the SM. At the low-energy scales $\mu \sim 100 \mathrm{MeV}$ typical for the $0 \nu 2 \mathrm{EC}$ process, the blobs are essentially pointlike, having been generated by the exchange of a heavy particle with the characteristic masses $M_{H}$ much larger than the $0 \nu 2 \mathrm{EC}$ scale, i.e., $M_{H} \gg \mu$. Integrating them out one finds the effective Lagrangian terms describing the vertices at the scale $\mu \ll$ $\Lambda \sim M_{H}$ for any kind of underlying high-scale physics beyond the SM. These vertices can be written in the following form (Päs et al., 1999, 2001):

$$
\begin{gathered}
\mathcal{L}_{q l}^{(6)}=\frac{G_{F}}{\sqrt{2}}\left(-j_{\mathrm{CC}}^{\mu} J_{\mathrm{CC} \mu}+\sum_{i} C_{i}^{X}(\mu) \mathcal{O}_{i}^{(6) X}(\mu)\right), \\
\mathcal{L}_{q l}^{(9)}=\frac{G_{F}^{2}}{2 m_{p}} \sum_{i, X Y} C_{i}^{X Y}(\mu) \mathcal{O}_{i}^{(9) X Y}(\mu) .
\end{gathered}
$$

The first and second lines correspond to Figs. 3(b) and 3(c), respectively. The proton mass $m_{p}$ is introduced to match the conventional notations. The complete set of the $\Delta L=2$ operators for $d=6$ and 9 is as follows (Arbeláez et al., 2016, 2017; González, Hirsch, and Kovalenko, 2016):

$$
\begin{gathered}
\mathcal{O}_{1}^{(6) X}=4\left(\bar{d} P_{X} u\right)\left(\overline{\nu^{c}} P_{L} e\right) \\
\mathcal{O}_{2}^{(6) X}=4\left(\bar{d} \sigma^{\mu \nu} P_{X} u\right)\left(\overline{\nu^{c}} \sigma^{\mu \nu} P_{L} e\right), \\
\mathcal{O}_{3}^{(6) X}=4\left(\bar{d} \gamma_{\mu} P_{X} u\right)\left(\overline{\nu^{c}} \gamma^{\mu} P_{R} e\right),
\end{gathered}
$$

$$
\begin{gathered}
\mathcal{O}_{1}^{(9) X Y}=4\left(\bar{d} P_{X} u\right)\left(\bar{d} P_{Y} u\right) j, \\
\mathcal{O}_{2}^{(9) X Y}=4\left(\bar{d} \sigma^{\mu \nu} P_{X} u\right)\left(\bar{d} \sigma_{\mu \nu} P_{X} u\right) j, \\
\mathcal{O}_{3}^{(9) X Y}=4\left(\bar{d} \gamma^{\mu} P_{X} u\right)\left(\bar{d} \gamma_{\mu} P_{Y} u\right) j, \\
\mathcal{O}_{4}^{(9) X Y}=4\left(\bar{d} \gamma^{\mu} P_{X} u\right)\left(\bar{u} \sigma_{\mu \nu} P_{Y} d\right) j^{\nu}, \\
\mathcal{O}_{5}^{(9) X Y}=4\left(\bar{d} \gamma^{\mu} P_{X} u\right)\left(\bar{d} P_{Y} u\right) j_{\mu},
\end{gathered}
$$

where $X, Y=L, R$ and the leptonic currents are $j=\overline{e^{c}}\left(1 \pm \gamma_{5}\right) e, j_{\mu}=\overline{e^{c}} \gamma_{\mu} \gamma_{5} e$. The first term in Eq. (2.1) describes the SM low-energy four-fermion effective interaction of the charged current $(\mathrm{CC})$ :

$$
j_{\mathrm{CC}}^{\mu}=\bar{\nu} \gamma^{\mu}\left(1-\gamma_{5}\right) e, \quad J_{\mathrm{CC} \mu}=\bar{d} \gamma_{\mu}\left(1-\gamma_{5}\right) u .
$$

The $\mathrm{SU}(3)_{c} \times \mathrm{U}(1)_{e m}$ symmetric operators in Eqs. (2.3)(2.10) are written in the mass-eigenstate basis. They originate from the $\mathrm{SU}(3)_{c} \times \mathrm{SU}(2)_{W} \times \mathrm{U}(1)_{Y}$ gauge invariant operators after the electroweak symmetry breaking; see Bonnet et al. (2013), Lehman (2014), and Graesser (2017).

Figures 3(a) and 3(b) are of second order in the Lagrangian (2.1). The effect of $\Delta L=2$ is introduced in Figs. 3(a) and 3(b) by the Majorana neutrino mass term and by the $d=6$ effective operators (2.3)-(2.5), respectively. Figure 3(a) is the conventional Majorana neutrino mass mechanism with the contribution to the $0 \nu 2 \mathrm{EC}$ amplitude

$$
V_{\alpha \beta} \sim m_{\beta \beta} \equiv \sum_{i} U_{e i}^{2} m_{\nu_{i}}
$$

where $U$ is the Pontecorvo-Maki-Nakagawa-Sakata (PMNS) mixing matrix and $m_{\beta \beta}$ is the effective electron neutrino Majorana mass parameter that is well known from the analysis of $0 \nu 2 \beta^{-}$decay. The contribution of Fig. 3(b) is independent of the neutrino mass but proportional to the momentum $q$ flowing in the neutrino propagator. This is the so-called $\hat{q}$-type contribution. The following comment is in order. Figures 3(a) and 3(b) show two possible mechanisms of both $0 \nu 2 \mathrm{EC}$ and $0 \nu 2 \beta^{-}$(with the inverted final to initial states) processes. The first mechanism in Fig. 3(a) contributes to the amplitude of these processes with terms proportional to the effective Majorana mass parameter $m_{\beta \beta}$ defined in Eq. (2.12). The contribution of the second mechanism in Fig. 3(b) has no explicit dependence on $m_{\beta \beta}$. This is because the upper vertex in Fig. 3(b) breaks the lepton number into two units, as necessary for this process to proceed without need of the $\Delta L=2$ Majorana neutrino mass insertion into the neutrino line. Note that $m_{\beta \beta}=0$ is compatible with the neutrino oscillation data in the case of normal neutrino mass ordering. This result shows that the mechanism in Fig. 3(a), proportional to $m_{\beta \beta}$, can be negligible in comparison to the mechanism in Fig. 3(b). Therefore, even if $m_{\beta \beta}$ turns out to be small, both the $0 \nu 2 \beta^{-}$decay and the $0 \nu 2 \mathrm{EC}$ process can be observable due to the latter mechanism. This possibility has been studied in the literature for $0 \nu 2 \beta^{-}$decay; see Päs et al. 


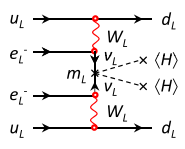

FIG. 4. The contribution of the Weinberg operator to the $0 \nu 2 \mathrm{EC}$ process in the flavor basis of the neutrino states.

(1999, 2001), Arbeláez et al. (2016, 2017), and González, Hirsch, and Kovalenko (2016).

The SM gauge invariant Weinberg dimension-5 effective operator generating the neutrino mass mechanism is given by (Weinberg, 1979)

$$
\begin{gathered}
\mathcal{L}_{W}^{(5)}=\kappa \frac{\left(\overline{L^{c}} H\right)(L H)}{\Lambda}=\frac{\kappa}{\Lambda} \overline{\nu^{c}} \nu H^{0} H^{0}+\cdots \\
\stackrel{\mathrm{SSB}}{\longrightarrow} \kappa \frac{\left\langle H^{0}\right\rangle^{2}}{\Lambda} \overline{\nu^{c}} \nu+\cdots .
\end{gathered}
$$

In Eq. (2.13) we mean $L H=L_{i} H_{j} \epsilon_{i j}$, the singlet combination of two $\mathrm{SU}(2)_{W}$ doublets $L\left(\overline{L^{c}}\right)$ and $H$. After the electroweak spontaneous symmetry breaking (SSB) with the Higgs vacuum expectation value $\langle H\rangle \neq 0$ neutrinos acquire a Majorana mass $m_{\nu}=-2 \kappa\langle H\rangle^{2} / \Lambda$, with $\kappa$ a dimensionless parameter. In the flavor basis of neutrino states the contribution of the Weinberg operator to a LNV process such as $0 \nu 2 \mathrm{EC}$ is displayed in Fig. 4. The summation of multiple insertions of the Weinberg operator into the bare neutrino propagator entails the renormalized neutrino propagator with Majorana mass $m_{\nu}$. The operator (2.13) is unique. Other operators of the effective Lagrangian are suppressed by higher powers of the unification scale $\Lambda$. The study of the neutrinoless 2EC process and $2 \beta^{-}$decays could be the most direct way of testing physics beyond the standard model. In terms of the naive dimensional counting one can expect the dominance of the Weinberg operator, with the dimension 5, over the operators of dimensions 6 and 9 in Eqs. (2.5) and (2.6). However, to set $m_{\nu}$ at $\mathrm{eV}$ scale one should provide a small coupling $\kappa \sim 10^{-11}$ for the phenomenologically interesting case of $\Lambda \sim O(1 \mathrm{TeV})$. However, the smallness of any dimensionless coupling requires explanation. Typically in this case one expects the presence of some underlying physics, for example, symmetry. The situation changes with the increase of the LNV scale up to $\Lambda \sim 10^{13-14}$, with $\kappa \sim 1$, where the contribution of the Weinberg operator to $0 \nu 2 \mathrm{EC}$ dominates. The final count depends on the concrete highscale underlying LNV model: not all operators appear in the low-energy limit and $\kappa$ is a small suppression factor allowing $\mathrm{TeV}$-scale $\Lambda$. The latter can stem from loops or the ratio of the SSB scales in multiscale models; for a recent analysis see Helo, Hirsch, and Ota (2016).

In this review the mechanism of the neutrino Majorana masses is discussed in detail, for which numerical evaluation of the neutrinoless 2EC half-lives of near-resonant nuclides with the known NMEs will be given. In the case of highdimensional operators, as well as for the $d=5$ mechanism with the unknown NMEs, normalized estimates are given that take into account the factorization of nuclear effects in the $0 \nu 2 \mathrm{EC}$ amplitude. Keeping the previous comments in mind, we also discuss mechanisms based on the operators of
Eqs. (2.5) and (2.6), leading to the contributions shown in Figs. 3(b) and 3(c).

The blobs in Figs. 3(b) and 3(c) can be opened up (ultraviolet completed) in terms of all possible types of renormalizable interactions consistent with the SM gauge invariance. These are the high-scale models, which lead to the $0 \nu 2 \mathrm{EC}$ process. A list of all the possible ultraviolet completions for $0 \nu 2 \beta^{-}$decay was given by Bonnet et al. (2013).

The Wilson coefficients $C_{i}$ in Eqs. (2.1) and (2.2) are calculable in terms of the parameters (couplings and masses) of a particular underlying model at the scale $\Lambda \sim M_{H}$, called the "matching scale." Note that some of $C_{i}(\Lambda)$ may vanish. To make contact with $0 \nu 2 \mathrm{EC}$ one needs to estimate $C_{i}$ at a scale $\mu_{0}$ close to the typical $0 \nu 2 \mathrm{EC}$-energy scale. The coefficients $C_{i}$ run from the scale $\Lambda$ down to $\mu_{0}$ due to the QCD corrections. In addition, the $d=9$ operators undergo the renormalization group equation mixing with each other, leading to the mixing of the corresponding Wilson coefficients.

The general parametrization of the $0 \nu 2 \mathrm{EC}$ amplitude derived from Fig. 3, taking into account the leading-order QCD running (González, Hirsch, and Kovalenko, 2016; Cirigliano et al., 2018a; Ayala, Cvetic, and Gonzalez, 2020; Liao, Ma, and Wang, 2020), reads

$$
\begin{aligned}
V_{\alpha \beta}= & G_{F}^{2} \cos ^{2} \theta_{C} K_{Z}\left(\sum_{i=1}^{3} \beta_{i}^{X}\left(\mu_{0}, \Lambda\right) C_{i}^{X}(\Lambda)\right. \\
& \left.+\sum_{i=1}^{5} \beta_{i}^{X Y}\left(\mu_{0}, \Lambda\right) C_{i}^{X Y}(\Lambda)\right) \mathcal{A}_{\alpha \beta} .
\end{aligned}
$$

The parameters $\beta_{i}^{X}$ and $\beta_{i}^{X Y}$ incorporate the QCD running of the Wilson coefficients and the matrix elements of the operators in Eqs. (2.3)-(2.5) combined with $j_{\mathrm{CC}}^{\mu} J_{\mathrm{CC} \mu}$ and the operators in Eqs. (2.6)-(2.10). The wave functions of the captured electrons with quantum numbers $\alpha$ and $\beta$ enter the coefficients $\mathcal{A}_{\alpha \beta}$ defined by Eqs. (2.20)-(2.23). In Eq. (2.14) the summation over the different chiralities $X, Y=L, R$ is implied. The Wilson coefficients $C_{i}(\Lambda)$ entering Eqs. (2.1) and (2.2) are linked to the matching scale $\Lambda$, where they are calculable in terms of the Lagrangian parameters of a particular high-scale underlying model. The decay amplitude (2.14) is supplemented by the overlap amplitude $K_{Z}$ of the electron shells of the initial and final atoms. In this review, we mainly discuss the light Majorana neutrino exchange mechanism of Fig. 3(a). The $0 \nu 2$ EC NMEs are currently known only for the Majorana neutrino exchange mechanisms coupled to left- and right-handed currents. Calculations of the NMEs corresponding to the other long- and short-range mechanisms of Figs. 3(b) and 3(c), respectively, for all operators (2.5) and (2.6) are still in progress.

\section{B. Examples of underlying high-scale models}

We give three examples of popular high-scale models that can underlie the $0 \nu 2 \mathrm{EC}$ process. In the low-energy limit their 
contribution is described by the effective Lagrangians (2.1) and/or (2.2).

Left-right symmetric models. - A well-known example of a high-scale model leading to $\Delta L=2$ processes, such as $0 \nu 2 \beta$ decay and $0 \nu 2 \mathrm{EC}$ and the generation of Majorana mass for neutrinos, is the left-right symmetric extension of the SM. The left-right symmetric model (LRSM) is based on the gauge group $\mathcal{G}$ spontaneously broken via the chain

$$
\begin{aligned}
\mathcal{G}=\mathrm{SU}(3)_{C} \times \mathrm{SU}(2)_{L} \times \mathrm{SU}(2)_{R} \times \mathrm{U}(1)_{B-L} \\
\Downarrow v_{R} \\
\mathrm{SU}(3)_{C} \times \mathrm{SU}(2)_{L} \times \mathrm{U}(1)_{Y} \\
\Downarrow v_{\mathrm{SM}} \\
\mathrm{SU}(3)_{C} \times \mathrm{U}(1)_{e m},
\end{aligned}
$$

where $v_{R} \equiv\left\langle\Delta_{R}\right\rangle \gg\langle\Phi\rangle \equiv v_{\mathrm{SM}}$ are the vacuum expectation values (VEVs) of a Higgs $\mathrm{SU}(2)_{R}$ triplet $\Delta_{R}$ and a Higgs bidoublet $\Phi$, respectively. The bidoublet belongs to the doublet representation of both $\mathrm{SU}(2)_{L}$ and $\mathrm{SU}(2)_{R}$. There is also a Higgs $\mathrm{SU}(2)_{L}$ triplet $\Delta_{L}$ with the $\mathrm{VEV} v_{L} \equiv\left\langle\Delta_{L}\right\rangle$. Left- and right-handed leptons and quarks belong to the doublet representations of the $\mathrm{SU}(2)_{L}$ and $\mathrm{SU}(2)_{R}$ gauge groups, respectively. The $\mathrm{SU}(3)_{C} \times \mathrm{SU}(2)_{L} \times \mathrm{SU}(2)_{R} \times$ $\mathrm{U}(1)_{B-L}$ assignments of the LRSM fields are

$$
\begin{aligned}
L_{L(R)} & =\left(\begin{array}{c}
\nu_{i} \\
\ell_{i}^{-}
\end{array}\right)_{L(R)} \sim[1,2(1), 1(2) ;-1], \\
Q_{L(R)} & =\left(\begin{array}{c}
u_{i} \\
d_{i}
\end{array}\right)_{L(R)} \sim[3,2(1), 2(1) ;-1 / 3], \\
\Delta_{L(R)} & =\left(\begin{array}{cc}
\frac{\Delta^{+}}{\sqrt{2}} & \Delta^{++} \\
\Delta^{0} & \frac{-\Delta^{+}}{\sqrt{2}}
\end{array}\right)_{L(R)} \sim[1,3(1), 1(3) ; 2], \\
\Phi & =\left(\begin{array}{ll}
\Phi_{1}^{0} & \Phi_{1}^{+} \\
\Phi_{2}^{-} & \Phi_{2}^{0}
\end{array}\right) \sim[1,2,2 ; 0],
\end{aligned}
$$

where $i=1,2,3$ is the generation index. Previously introduced VEVs are related to the VEVs of the electrically neutral components $\left\langle\Delta_{L, R}\right\rangle \equiv\left\langle\Delta_{L, R}^{0}\right\rangle,\langle\Phi\rangle^{2}=\left\langle\Phi_{1}^{0}\right\rangle^{2}+\left\langle\Phi_{2}^{0}\right\rangle^{2} \equiv v_{\mathrm{SM}}$. There are two charged gauge bosons $W_{L, R}^{ \pm}$and two neutral gauge bosons $Z_{L, R}$ with masses of the order of $M_{W_{R}}, M_{Z_{R}} \sim g_{R} v_{R}, M_{W_{L}}, M_{Z_{L}} \sim g_{L} v_{\mathrm{SM}}$. Note that in the scenario with the manifest left-right symmetry the $\mathrm{SU}(2)_{L, R}$ gauge couplings obey $g_{L}=g_{R}$. Since the bosons $W_{R}, Z_{R}$ have not been experimentally observed, the scale of the left-right symmetry breaking $v_{R}$ must be sufficiently large, above a few TeV. On the other hand, the VEV of the "left" triplet $v_{L}$ must be small since it affects the SM relation $\rho=1$, which is in good agreement with the experimental measurements setting an upper limit $v_{L} \lesssim 8 \mathrm{GeV}$. From the scalar potential of the LRSM follows $v_{L} \sim v_{S \mathrm{M}}^{2} / v_{R}$, which satisfies the previously mentioned upper limit for $v_{R} \gtrsim 10 \mathrm{TeV}$.

The spontaneous gauge symmetry breaking (2.15) generates a $6 \times 6$ neutrino seesaw-I mass matrix given in the basis $\left(\nu_{L}, \nu_{R}^{C}\right)^{T}$ by

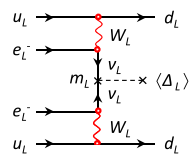

(a)

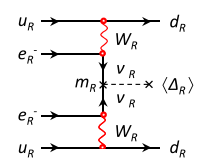

(b)

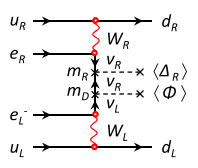

(c)

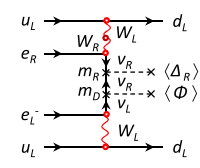

(d)

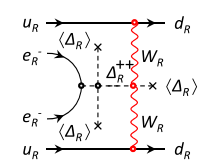

(e)
FIG. 5. Possible flavor-basis contributions to $0 \nu 2 \mathrm{EC}$ within the LRSM.

$$
M^{\nu}=\left(\begin{array}{ll}
m_{L} & m_{D} \\
m_{D}^{T} & m_{R}
\end{array}\right),
$$

with $m_{L, R} \sim y_{L, R} v_{L, R}$ and $m_{D} \sim y_{\Phi} v_{\mathrm{SM}} \quad 3 \times 3$ block matrices in the generation space. The matrix (2.17) is diagonalized to $U^{T} M^{\nu} U=\operatorname{diag}\left(m_{\nu_{i}} ; m_{N_{i}}\right)$ by an orthogonal mixing matrix

$$
U=\left(\begin{array}{cc}
U_{L} & U_{D} \\
U_{D}^{T} & U_{R}
\end{array}\right)
$$

with $U_{L, R, D} 3 \times 3$ block matrices in the generation space. The neutrino mass spectrum consists of three light $\nu_{1,2,3}$ and three heavy $N_{1,2,3}$ Majorana neutrino states with the masses $m_{\nu_{1,2,3}}$ and $m_{N_{1,2,3}}$, respectively.

The possible contributions to $0 \nu 2 \mathrm{EC}$ within the LRSM are shown in Fig. 5.

Figure 5(a) shows the conventional long-range light Majorana neutrino exchange mechanisms with the contribution shown in Eq. (2.12). Figures 5(b) and 5(e) are short-range mechanisms with two heavy right-handed bosons $W_{R}$ and heavy neutrino $\nu_{R}$ or doubly charged Higgs $\Delta^{++}$exchange. In the low-energy limit they reduce to the effective operators $\mathcal{O}_{3}^{(9) R R}$ in Eq. (2.8) depicted in Fig. 3(c). Figures 5(c) and 5(d), containing light virtual neutrinos, represent the long-range mechanism of $0 \nu 2 \mathrm{EC}$.

In the low-energy limit the upper parts of Figs. 5(c) and 5(d) with heavy particles $W_{R}$ and $\nu_{R}$ reduce to the $d=6$ effective operators $\mathcal{O}_{3}^{(6) R}$ and $\mathcal{O}_{3}^{(6) L}$, respectively. Note that these contributions to the $0 \nu 2 \mathrm{EC}$ amplitude depend not on the light neutrino mass $m_{\nu}$ but on its momentum $\mathbf{q}$ flowing in the neutrino propagator. Technically this happens because different chiralities of the lepton vertices project the $\hat{q}$ term out of the neutrino propagator $P_{L}\left(\hat{q}+m_{\nu}\right) P_{R}=P_{L} \hat{q}$. On the contrary, Fig. 5(a), with the same chiralities in both vertices, is proportional to $m_{\nu}$ due to $P_{L}\left(\hat{q}+m_{\nu}\right) P_{L}=P_{L} m_{\nu}$. This is consistent with the fact that in the latter case the source of LNV is the Majorana neutrino mass $m_{\nu}$ and in the limit $m_{\nu} \rightarrow 0$ the corresponding contribution must vanish. On the other hand, in the former case [Figs. 5(c) and 5(d)] the LVN source is the operator in the upper vertex and $m_{\nu}$ is not needed 
to allow for the $\Delta L=2$ process to proceed. These are the so-called $\hat{q}$-type contributions.

The Wilson coefficients $C_{n}^{K}$ in Eqs. (2.2) and (2.14) at the matching high-energy scale $\Lambda \sim M_{R}$ corresponding to Figs. 5(b)-5(e) are given by

Fig.5(b) $\sim y_{R}\left\langle\Delta_{R}\right\rangle \rightarrow C_{3}^{R R, N_{R}}=\sum_{i=1}^{3} U_{L e i}^{2} \frac{m_{p}}{m_{N_{i}}}\left(\frac{M_{W_{L}}}{M_{W_{R}}}\right)^{4}$,

Fig.5(c) $\sim y_{\Phi} y_{R}\langle\Phi\rangle\left\langle\Delta_{R}\right\rangle \rightarrow C_{3}^{R, \hat{q}}=\sum_{i=1}^{3} U_{L e i} U_{D, e i}\left(\frac{M_{W_{L}}}{M_{W_{R}}}\right)^{2}$,

Fig.5(d) $\zeta_{W} y_{\Phi} y_{R}\langle\Phi\rangle\left\langle\Delta_{R}\right\rangle \rightarrow C_{3}^{L, \hat{q}}=\sum_{i=1}^{3} U_{L e i} U_{D, e i} \tan \zeta_{W}$,

Fig.5(e) $\sim \lambda_{\Delta_{R}} g_{R}^{2}\left\langle\Delta_{R}\right\rangle \rightarrow C_{3}^{R R, \Delta_{R}^{++}}=\sum_{i=1}^{3} U_{R e i}^{2} \frac{m_{N_{i}} m_{p}}{m_{\Delta_{R}}^{2}}\left(\frac{M_{W_{L}}}{M_{W_{R}}}\right)^{4}$.

Here $\zeta_{W}$ is the angle of $W_{L}-W_{R}$ mixing. For convenience we show the correspondence of the flavor-basis diagrams in Fig. 5 to the particular combinations of the parameters of the LRSM Lagrangian (quartic $\Delta_{R}$ coupling $\lambda_{\Delta_{R}}$, gauge coupling $g_{R}$, and $\operatorname{VEV}\left\langle\Delta_{R}\right\rangle$ ), then give the corresponding Wilson coefficients.

Leptoquark models.-Leptoquarks (LQs) are exotic scalar or vector particles coupled to lepton-quark pairs in such a way that $\bar{L}(L Q) Q$. They appear in various high-scale contexts, for example, in grand unification, extended technicolor, compositeness, etc. For a generic LQ theory all the renormalizable interactions were specified by Buchmüller, Rückl, and Wyler (1987). Current experimental limits (Tanabashi et al., 2018) allow them to be relatively light at the TeV scale. The SM gauge symmetry allows LQs to mix with the SM Higgs. This mixing generates $\Delta L=2$ interactions with the chiral structure leading to the long-range $\hat{q}$-type contribution not suppressed by the smallness of the Majorana mass $m_{\nu}$ of the light virtual neutrino displayed in Fig. 6(a), with $S$ or $V$ being scalar or vector LQs. In the low-energy limit, the upper part of Fig. 6(a) with heavy LQs reduces to the pointlike vertex described by the operator $\mathcal{O}_{1}^{(6) X}$ in Eq. (2.3). The chirality structure of this vertex combined with the SM vertex in the bottom part of Fig. 6(a) render a $\hat{q}$-type contribution to $0 \nu 2 \mathrm{EC}$.

$R$-parity violating supersymmetric models.-The TeV-scale supersymmetric (SUSY) models offer a natural explanation of the grand unified theory-SM scale hierarchy, introducing superpartners to each SM particle, so that they form supermultiplets (chiral superfields): $(q, \tilde{q}),(l, \tilde{l}),(g, \tilde{g})$, etc. Here $\tilde{q}$ and $\tilde{l}$ are scalar squarks and sleptons, while $\tilde{g}$ is a spin- $1 / 2$ gluino. The SUSY framework requires at least two electroweak Higgs doublets $H_{U}$ and $H_{D}$. A class of SUSY models, the so-called $R$-parity violating (RPV) SUSY models, allow for LNV interactions described by a superpotential

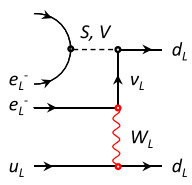

(a)

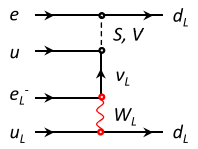

(b)

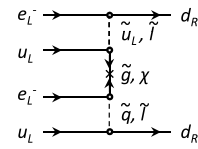

(c)
FIG. 6. Possible flavor-basis contributions to $0 \nu 2 \mathrm{EC}$ within (a),(b) leptoquark and (a),(c) RPV MSSM models. The cross in (c) denotes the Majorana mass of a gluino $\tilde{g}$ or neutralino $\chi$.

$$
W_{\mathrm{RPV}}=\lambda_{i j k}^{\prime} L_{i} Q_{j} \bar{D}_{k}+\epsilon_{i} L_{i} H_{U},
$$

where $Q, D$, and $H_{U}$ conventionally denote here the chiral superfields of the left-chiral electroweak doublet quarks, the right-chiral electroweak singlet down quark, and the up-type electroweak Higgs doublet, respectively.

The RPV SUSY models with the interactions (2.23) contribute to the long- and short-range mechanisms of the $0 \nu 2 \mathrm{EC}$ process. The corresponding diagrams are shown in Fig. 6 . Figures 6(a) and 6(b) generate the long-range $\hat{q}$-type contribution, while Fig. 6(c) with the gluino $\tilde{g}$ or neutralino $\chi$ exchange entails the short-range contribution. The source of the lepton number violation is located in the vertices of Figs. 6(a) and 6(b), while in Fig. 6(c) it is given by the Majorana mass of the neutralino $m_{\chi}$ and/or gluino $m_{\tilde{g}}$. In the low-energy limit Figs. 6(a) and 6(b) lead to the operator $\mathcal{O}_{1}^{(6) L}$, while in this limit Fig. 6(c), where all internal particles are heavy, collapses to a pointlike short-range contribution given by a linear combination of $d=9$ operators $\mathcal{O}_{1}^{(9) L L}$ and $\mathcal{O}_{2}^{(9) L L}$.

\section{Hadronization of quark-level interactions}

We now comment on the calculation of the structure coefficients $\beta_{i}^{X}, \beta_{i}^{X Y}$ in the amplitude (2.14) depending on the NMEs and nucleon structure. The Lagrangians (2.1) and (2.2) can, in principle, be applied to any LNV processes with whatever hadronic states: quarks, mesons, nucleons, and other baryons, as well as nuclei. The corresponding amplitude, such as that in Eq. (2.14), involves the hadronic matrix elements of the operators (2.6) and (2.5). The Wilson coefficients $C_{i}^{X Y}$ are calculated in terms of the parameters of the high-scale model and are independent of the low-energy scale nonperturbative hadronic dynamics. This is the celebrated property of the operator product expansion, expressing interactions of some high-scale renormalizable model in the form of Eqs. (2.1) and (2.2) below a certain scale $\mu$. In the case of $0 \nu 2 \beta^{-}$decay, $0 \nu 2 \mathrm{EC}$, and other similar nuclear processes, the corresponding NMEs of the operators (2.6) and (2.5) are calculated in the framework of the approach based on a nonrelativistic impulse approximation; for a detailed description see Doi, Kotani, and Takasugi (1985). This implies, as the first step, reformulation of the quark-level theory in terms of the nucleon degrees of freedom, which the existing nuclear-structure models operates with. This is the socalled hadronization procedure. In the absence of a firm theory of hadronization one is left to resort upon general principles and particular models. Imbedding two initial (final) quarks into two different protons (neutrons) is conceptually a more 


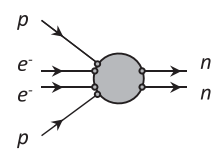

(a)

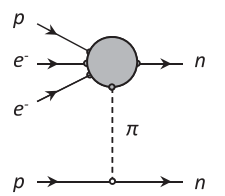

(b)

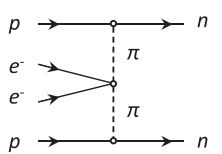

(c)
FIG. 7. Hadron-level diagrams for $0 \nu 2$ EC. (a) Conventional two-nucleon mechanism. (b) One-pion exchange mechanism. (c) Two-pion exchange mechanism.

simple option that is illustrated in Fig. 7(a). This is the conventional two-nucleon mechanism relying on the nucleon form factors as a phenomenological representation of the nucleon structure. On the other hand, putting one initial and one final quark into a charged pion while the other initial quark is put into a proton and one final quark into a neutron, as in Fig. 7(b), we deal with the one-pion mechanism. The twopion mechanism, displayed in Fig. 7(c), treats all quarks to be incorporated in two charged pions. In both cases the pions are virtual and interact with nucleons via the ordinary pseudoscalar pion-nucleon coupling $\bar{N} i \gamma_{5} \tau \pi N$. One may expect a priori dominance of the pion mechanism for the reason that it extends the region of the nucleon-nucleon interaction due to the smallness of the pion mass leading to a long-range potential. As a result, the suppression caused by the shortrange nuclear correlation can be significantly alleviated in comparison to the conventional two-nucleon mechanism. Nevertheless, one should consider all these mechanisms to contribute to the process in question with corresponding relative amplitudes. The latter is as yet unknown. In principle, it can be evaluated in particular hadronic models. These kinds of studies are still missing in the literature and consensus on the dominance of one of the two mechanisms is pending. It is a common trend to posit an analysis of one of the two hadronization scenarios. Note that for the long-range contributions described by the effective Lagrangian (2.1) the previously mentioned advantage of the pion mechanism is absent and one can, in a sense, safely resort to the conventional two-nucleon mechanism. The light Majorana exchange contribution to $0 \nu 2 \mathrm{EC}$, on which we focus in the rest of this review, is of this kind. This limitation is explained by the fact that for the moment there are no nuclear matrix elements yet calculated in the literature for mechanisms other than this one.

The procedure of hadronization is essentially the same as for $0 \nu \beta \beta$ decay and is described in the literature. For more details on this approach to hadronization see Doi, Kotani, and Takasugi (1985), Faessler, Kovalenko, and Šimkovic (1998), Faessler et al. (2008), and Graf et al. (2018).

Recently another approach has been developed, which resorts to matching the high-scale quark-level theory to chiral perturbation theory. The latter is believed to provide a lowenergy description of QCD in terms of nucleon and pion degrees of freedom. It is expected that the parameters of the low-energy effective theory can be determined from experimental measurements or from the lattice QCD. This approach leads to a different picture of hadronization and numerical results compared to the previously sketched conventional approach. Contrary to the conventional approach short-range nucleon-nucleon interactions should be introduced for theoretical self-consistency even in the case of the long-range light neutrino exchange mechanism in Fig. 3(a). For a detailed description of this approach see Prezeau, Ramsey-Musolf, and Vogel (2003), Cirigliano et al. (2017a, 2017b, 2018a, 2018b, 2019, 2020), and Graesser (2017).

To conclude, neutrinoless double-electron capture $0 \nu 2 \mathrm{EC}$, like $0 \nu \beta \beta$ decay, is a $\Delta L=2$ lepton number violating process. Moreover, on the level of a nucleon subprocess it is virtually equal to $0 \nu \beta \beta$ decay. Consequently, the underlying $\Delta L=2$ physics driving both these processes is the same. There are many formal differences in the form of the effective operators representing this physics at low-energy sub-GeV scales. We specified a complete basis of the $0 \nu 2 \mathrm{EC}$ effective operators in Eqs. (2.1)-(2.10) and exemplified high-energy scale models presently popular in the literature, which can be reduced to these operators in the low-energy limit. Akin to $0 \nu \beta \beta$ decay there are basically three types of mechanisms of $0 \nu 2 \mathrm{EC}$ shown in Fig. 3: Fig. 3(a) shows the conventional neutrino exchange mechanism with the amplitude proportional to the effective Majorana neutrino mass $m_{\beta \beta}$ defined in Eq. (2.12), and Fig. 3(b) displays a neutrino exchange mechanism independent of the Majorana neutrino mass, when the lepton number violation necessary for $0 \nu 2 \mathrm{EC}$ to proceed is gained from a $\Delta L=2$ vertex. Figures 3(a) and 3(b) are both long-range mechanics induced by the exchange of an extremely light particle, a neutrino. On the other hand, Fig. 3(c) represents a short-range mechanism induced by the exchange of heavy particles with masses much larger than the typical scale (approximately a few $\mathrm{MeV}$ ) of $0 \nu 2 \mathrm{EC}$. Despite the underlying physics of both $0 \nu 2 \mathrm{EC}$ and $0 \nu \beta \beta$ decay being the same, their NMEs are significantly different. We discuss the nuclearstructure aspects and atomic physics involved in the calculations of the $0 \nu 2 \mathrm{EC}$ NMEs in subsequent sections. Here it is worth noting that thus far only the NMEs for the Majorana neutrino exchange mechanism in Fig. 3(a) have been calculated in the literature. Similar calculations for NMEs of other mechanisms in Figs. 3(b) and 3(c) are still pending.

\section{PHENOMENOLOGY OF NEUTRINOLESS 2EC}

Figures 1 and 2 can be combined as shown in Fig. 8. In the initial state, there is an atom $(A, Z)$. The electron lines belong to the electron shells, and the proton and neutron lines belong to the initial and intermediate nuclei, respectively. As a result of neutrinoless double-electron capture, an atom $(A, Z-2)^{* *}$ is formed, generally in an excited state. In what follows, $(A, Z)^{*}$ denotes an atom with the excited electron shell and $(A, Z)^{* *}$ indicates that the nucleus is also excited. The intermediate atom can decay by emitting a photon or Auger electrons, but it can also experience $0 \nu 2 \beta^{-}$transition and evolve back to the initial state. As a result, LNV oscillations $(A, Z) \leftrightarrow(A, Z-2)^{* *}$ occur in the two-level system. These oscillations are affected by the coupling of the $(A, Z-2)^{* *}$ atom to the continuum, which eventually leads to the decay of $(A, Z)$. The Hamiltonian of the system is not Hermitian because $(A, Z-2)^{* *}$ has a finite width.

The LNV oscillations of atoms were discussed by Šimkovic and Krivoruchenko (2009), Krivoruchenko et al. (2011), and Bernabéu and Segarra (2018). The formalism of LNV 


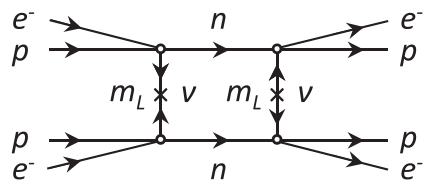

$(A, Z) \leftrightarrow(A, Z-2)^{* *} \leftrightarrow(A, Z)$

FIG. 8. Oscillations of atoms induced by $0 \nu 2 \mathrm{EC}$ and $0 \nu 2 \beta$ transitions. The notations are the same as in Figs. 1 and 2. The intermediate atom $(A, Z-2)^{* *}$ is coupled to the continuum through the emission of a photon or Auger electrons. These channels generate a finite width $\Gamma_{f}$ in Eq. (3.1), and they are also responsible for the non-Hermitian character of $H_{\mathrm{eff}}$.

oscillations allows one to find a relationship between the halflife $T_{1 / 2}$ of the initial atom $(A, Z)$, the amplitude of neutrinoless double-electron capture $V_{\alpha \beta}$, and the decay width of the intermediate atom $(A, Z-2)^{* *}$, which has an electromagnetic origin.

\section{A. Underlying formalism}

The evolution of a system of mixed states, each of which may be unstable due to the coupling with the continuum, can described by an effective non-Hermitian Hamiltonian (Weisskopf and Wigner, 1930). In the case under consideration, the Hamiltonian takes the form

$$
H_{\mathrm{eff}}=\left(\begin{array}{cc}
M_{i} & V_{\alpha \beta} \\
V_{\alpha \beta}^{*} & M_{f}-\frac{i}{2} \Gamma_{f}
\end{array}\right)
$$

where $M_{i}$ and $M_{f}$ are the masses of the initial and final atoms. The width $\Gamma_{f}$ of the final excited atom with two vacancies $\alpha$ and $\beta$ is of electromagnetic origin. The off-diagonal matrix elements are due to a violation of lepton number conservation. They can be chosen as real by changing the phase of one of the states; thus, we set $V_{\alpha \beta}=V_{\alpha \beta}^{*}$. The real and imaginary parts of the Hamiltonian do not commute.

We now find the evolution operator

$$
U(t)=\exp \left(-i H_{\mathrm{eff}} t\right)
$$

According to Sylvester's theorem, the function of a finitedimensional $n \times n$ matrix $A$ is expressed in terms of the eigenvalues $\lambda_{k}$ of the matrix $A$, which are solutions of the characteristic equation $\operatorname{det}(A-\lambda)=0$, and a polynomial of $A$ :

$$
f(-i A t)=\sum_{k} f\left(-i \lambda_{k} t\right) \prod_{l \neq k} \frac{\lambda_{l}-A}{\lambda_{l}-\lambda_{k}}
$$

where the sum runs over $1 \leq k \leq n$, the product runs over $1 \leq l \leq n, l \neq k$, and the eigenvalues are assumed to be pairwise distinct. The matrix function $f(-i A t)$ evolves with the time $t$ like the superposition of $n$ terms $f\left(-i \lambda_{k} t\right)$ with the matrix coefficients that are projection operators onto the $k$ th eigenstates of $A$.

The eigenvalues of the Hamiltonian (3.1) are equal to $\lambda_{ \pm}=M_{+} \pm \Omega$, where $M_{ \pm}=\left(M_{i} \pm M_{f}\right) / 2 \mp i \Gamma_{f} / 4$ and
$\Omega=\sqrt{V_{\alpha \beta}^{2}+M_{-}^{2}}$. The values of $\lambda_{ \pm}$are complex, so the norm of the states is not preserved in time. A series expansion around $V=0$ yields

$$
\begin{gathered}
\lambda_{+} \approx M_{i}+\Delta M-\frac{i}{2} \Gamma_{i}, \\
\lambda_{-} \approx M_{f}-\Delta M-\frac{i}{2}\left(\Gamma_{f}-\Gamma_{i}\right),
\end{gathered}
$$

with $\Delta M=\left(M_{i}-M_{f}\right) \Gamma_{i} / \Gamma_{f}, \Gamma_{i}=V_{\alpha \beta}^{2} R_{f}$, and

$$
R_{f}=\frac{\Gamma_{f}}{\left(M_{i}-M_{f}\right)^{2}+(1 / 4) \Gamma_{f}^{2}} .
$$

The initial state decays at the rate $\Gamma_{i} \ll \Gamma_{f}$. The width $\Gamma_{i}$ is maximal for complete degeneracy of the atomic masses:

$$
\Gamma_{i}^{\max }=\frac{4 V_{\alpha \beta}^{2}}{\Gamma_{f}}
$$

A simple calculation gives

$U(t)=\exp \left(-i M_{+} t\right)\left(\cos (\Omega t)-i \frac{H_{\mathrm{eff}}-M_{+}}{\Omega} \sin (\Omega t)\right)$.

The decay widths of single-hole excitations of atoms are known experimentally and were tabulated for $10 \leq Z \leq 92$ and principal quantum numbers $1 \leq n \leq 4$ by Campbell and Papp (2001). The width of a two-hole state $\alpha \beta$ is represented by the sum of the widths of the one-hole states $\Gamma_{f}=\Gamma_{\alpha}+\Gamma_{\beta}$. The deexcitation width of the daughter nucleus is much smaller and can be neglected. The values $\Gamma_{f}$ are used in estimating the decay rates $\Gamma_{i}$.

The transition amplitude from the initial to the final state for small time $t$, according to Eq. (3.8), is equal to

$$
\langle f|U(t)| i\rangle=-i V_{\alpha \beta} t+\cdots .
$$

Equation (3.9) is valid for $t \lesssim 1 /\left|M_{+}\right|$and also over a wider range $t \lesssim 1 /|\Omega|$, given that the real part of the phase can be made to vanish via redefinition of the Hamiltonian $H_{\text {eff }} \rightarrow H_{\text {eff }}-\Re\left(M_{+}\right)$. The value of $V_{\alpha \beta}$ can be evaluated by means of field-theoretical methods that allow one to find the amplitude (3.9) from first principles. The formalism described in this section reproduces the results of Bernabeu, De Rujula, and Jarlskog (1983) with respect to $0 \nu 2 \mathrm{EC}$ decay rates.

\section{B. Decay amplitude of the light Majorana neutrino exchange mechanism}

The total lepton number violation is due to the Majorana masses of the neutrinos. It is assumed that the left electron neutrino is a superposition of three left Majorana neutrinos:

$$
\nu_{e L}=\sum_{i=1}^{3} U_{e k} \chi_{k L}
$$


where $U$ is the PMNS mixing matrix. In the Majorana bispinor representation, $\chi_{k L}=(1 / 2)\left(1-\gamma_{5}\right) \chi_{k}$ and $\chi_{k}^{*}=\chi_{k}$. The vertex describing the creation and annihilation of a neutrino has the standard form

$$
\mathcal{H}(x)=\frac{G_{F} \cos \theta_{C}}{\sqrt{2}} j_{\mu}(x) J_{h}^{\mu}(x)+\text { H.c. },
$$

where $\theta_{C}$ is the Cabibbo angle. The lepton and quark charged currents are defined by Eq. (2.11). In terms of the composite fields, the hadron charged current is given by

$$
J_{h}^{\mu}(x)=\bar{n}(x) \gamma^{\mu}\left(g_{V}-g_{A} \gamma_{5}\right) p(x),
$$

where $n(x)$ and $p(x)$ are the neutron and proton field operators and $g_{V}=1$ and $g_{A}=1.27$ are the vector and axialvector coupling constants, respectively. An effective theory could also include $\Delta$ isobars, meson fields, and their vertices for decaying into lepton pairs and interacting with nucleons and each other.

As a result of the capture of electrons, the nucleus $(A, Z)$ undergoes a $0^{+} \rightarrow J^{\pi}$ transition. Conservation of total angular momentum requires that the captured electron pair be in the state $J$. In weak interactions, parity is not conserved; thus, it is not required that the parity of the electron pair be correlated with the parity of the daughter nucleus.

The wave function of a relativistic electron in a central potential has the form

$$
\Psi_{\alpha m_{\alpha}}(\mathbf{r})=\left(\begin{array}{c}
f_{\alpha}(r) \Omega_{\alpha m_{\alpha}}(\mathbf{n}) \\
i g_{\alpha}(r) \Omega_{\alpha^{\prime} m_{\alpha}}(\mathbf{n})
\end{array}\right),
$$

where $\quad \alpha=(n 2 j l), \quad \alpha^{\prime}=\left(n 2 j l^{\prime}\right), \quad$ and $\quad l^{\prime}=2 j-l$. The radial wave functions are defined in agreement with Berestetsky, Lifshitz, and Pitaevsky (1982); $\Omega_{\alpha m_{\alpha}}(\mathbf{n}) \equiv$ $\Omega_{j m_{\alpha}}^{l}(\mathbf{n}), \Omega_{\alpha^{\prime} m_{\alpha}}(\mathbf{n})=\Omega_{j m_{\alpha}}^{l^{\prime}}(\mathbf{n})$ are spherical spinors in the notation of Varshalovich, Moskalev, and Khersonskii (1988). The normalization condition for $\Psi_{\alpha m_{\alpha}}(\mathbf{r})$ is given by

$$
\int d \mathbf{r} \Psi_{\alpha m_{\alpha}}^{\dagger}(\mathbf{r}) \Psi_{\beta m_{\beta}}(\mathbf{r})=\delta_{\alpha \beta} \delta_{m_{\alpha} m_{\beta}} .
$$

If the captured electrons occupy the states $\alpha \equiv(n 2 j l)_{1}$ and $\beta \equiv(n 2 j l)_{2}$, we must take the superposition of products of their wave functions:

$$
\psi_{\alpha \beta}^{J M}\left(\mathbf{r}_{1}, \mathbf{r}_{2}\right)=\sum_{m_{\alpha} m_{\beta}} C_{j_{\alpha} m_{\alpha} j_{\beta} m_{\beta}}^{J M} \Psi_{\alpha m_{\alpha}}\left(\mathbf{r}_{1}\right) \Psi_{\beta m_{\beta}}\left(\mathbf{r}_{2}\right),
$$

where $j_{\alpha}$ and $j_{\beta}$ are the total angular momenta, $m_{\alpha}$ and $m_{\beta}$ are their projections on the direction of the $z$ axis, and $\Psi_{\alpha m_{\alpha}}(\mathbf{r})$ and $\Psi_{\beta m_{\beta}}(\mathbf{r})$ are the relativistic wave functions of the bound electrons in an electrostatic mean field of the nucleus and the surrounding electrons. The identity of the fermions implies that the wave function of two fermions is antisymmetric; thus, the final expression for the wave function takes the form

$\Psi_{\alpha \beta}^{J M}\left(\mathbf{r}_{1}, \mathbf{r}_{2}\right)=\mathcal{N}_{\alpha \beta}\left[\Psi_{\alpha \beta}^{J M}\left(\mathbf{r}_{1}, \mathbf{r}_{2}\right)-(-)^{j_{\alpha}+j_{\beta}-J} \Psi_{\beta \alpha}^{J M}\left(\mathbf{r}_{1}, \mathbf{r}_{2}\right)\right]$,

where $\mathcal{N}_{\alpha \beta}$ equals $1 / \sqrt{2}$ for $\alpha \neq \beta$ and $1 / 2$ for $\alpha=\beta$.

As a consequence of the identity $C_{j m_{1} j m_{2}}^{J M}=(-1)^{J-2 j} C_{j m_{2} j m_{1}}^{J M}$, the wave function of two electrons with equal quantum numbers $\alpha=\beta$ is symmetric under the permutation $m_{\alpha} \leftrightarrow$ $m_{\beta}$ provided that their angular momenta are combined to the total angular momentum $J=2 j \bmod (2)$. In such a case, the antisymmetrization (3.16) yields zero, which means that the states $J=2 j \bmod (2)$ are nonexistent. The antisymmetrization (3.16) of the states $J=2 j+1 \bmod (2)$ leads to a doubling of the initial wave function. To keep the norm, the additional factor $1 / \sqrt{2}$ is thus required for $\alpha=\beta$.

The derivation of the equation for $V_{\alpha \beta}$ is analogous to the corresponding derivation of the $0 \nu 2 \beta$ decay amplitude, as described by Bilenky and Petcov (1987). The specificity is that a transition from a discrete level to a quasidiscrete level is considered. Accordingly, the delta function expressing the energy conservation is replaced by a time interval that can be identified with the parameter $t$ in Eq. (3.9). We thus write

$$
\langle f|\mathcal{U}(t)| i\rangle=-i V_{\alpha \beta} t+\cdots,
$$

where $\mathcal{U}(t)$ is Dyson's $U$ matrix. The amplitude takes the form

$$
\begin{aligned}
V_{\alpha \beta}= & i K_{Z} m_{\beta \beta} \sqrt{2} \mathcal{N}_{\alpha \beta}\left(\frac{G_{F} \cos \theta_{C}}{\sqrt{2}}\right)^{2} \int \frac{d \mathbf{q}}{(2 \pi)^{3}} d \mathbf{r}_{1} d \mathbf{r}_{2} \frac{1}{\sqrt{2 J+1}} \sum_{M} \frac{e^{-i \mathbf{q} \cdot\left(\mathbf{r}_{1}-\mathbf{r}_{2}\right)}}{2 q_{0}} \\
& \times\left[T_{\mu \nu \alpha \beta}^{J M}\left(\mathbf{r}_{1}, \mathbf{r}_{2}\right) N_{J M \alpha \beta}^{\mu \nu}\left(\mathbf{r}_{1}, \mathbf{r}_{2}\right)-(-1)^{j_{\alpha}+j_{\beta}-J} T_{\mu \nu \beta \alpha}^{J M}\left(\mathbf{r}_{1}, \mathbf{r}_{2}\right) N_{J M \beta \alpha}^{\mu \nu}\left(\mathbf{r}_{1}, \mathbf{r}_{2}\right)\right],
\end{aligned}
$$

where

$$
\begin{aligned}
T_{\mu \nu \alpha \beta}^{J M}\left(\mathbf{r}_{1}, \mathbf{r}_{2}\right) & =\sum_{m_{\alpha} m_{\beta}} C_{j_{\alpha} m_{\alpha} j_{\beta} m_{\beta}}^{J M}\left[\bar{\Psi}_{\alpha m_{\alpha}}^{c}\left(\mathbf{r}_{1}\right) \gamma_{\mu}\left(1+\gamma_{5}\right) \gamma_{\nu} \Psi_{\beta m_{\beta}}\left(\mathbf{r}_{2}\right)\right], \\
N_{J M \alpha \beta}^{\mu \nu}\left(\mathbf{r}_{1}, \mathbf{r}_{2}\right) & =\sum_{n}\left[\frac{\left\langle J M\left|J_{h}^{\mu}\left(\mathbf{r}_{1}\right)\right| n\right\rangle\left\langle n\left|J_{h}^{\nu}\left(\mathbf{r}_{2}\right)\right| 00\right\rangle}{q_{0}+E_{n}-M_{i}-\varepsilon_{\beta}}+\frac{\left\langle J M\left|J_{h}^{\nu}\left(\mathbf{r}_{2}\right)\right| n\right\rangle\left\langle n\left|J_{h}^{\mu}\left(\mathbf{r}_{1}\right)\right| 00\right\rangle}{q_{0}+E_{n}-M_{i}-\varepsilon_{\alpha}}\right] .
\end{aligned}
$$

Here $q_{0} \approx|\mathbf{q}|,\langle J M|=\langle f|$ and $|00\rangle=|i\rangle$ are the states of the final and initial nuclei, respectively; $\varepsilon_{\gamma}=m-\varepsilon_{\gamma}^{*}$, where $\varepsilon_{\gamma}^{*}$ is the one-hole excitation energy of the initial atom. The sum is taken over all excitations of the intermediate atom $(A, Z-1)$. In the Majorana bispinor representation, $\Psi_{\alpha m_{\alpha}}^{c}=\Psi_{\alpha m_{\alpha}}^{*}$. The amplitude $V_{\alpha \beta}$ is a scalar under rotation. By virtue of identities 


$$
\begin{aligned}
T_{\mu \nu \alpha \beta}^{J M}\left(\mathbf{r}_{1}, \mathbf{r}_{2}\right) & =-(-1)^{j_{\alpha}+j_{\beta}-J} T_{\nu \mu \beta \alpha}^{J M}\left(\mathbf{r}_{2}, \mathbf{r}_{1}\right), \\
N_{J M \alpha \beta}^{\mu \nu}\left(\mathbf{r}_{1}, \mathbf{r}_{2}\right) & =N_{J M \beta \alpha}^{\nu \mu}\left(\mathbf{r}_{2}, \mathbf{r}_{1}\right),
\end{aligned}
$$

$V_{\alpha \beta}$ is also invariant under permutations of $\alpha$ and $\beta$. For $\alpha=\beta$ and $J=2 j_{\alpha}+1 \bmod (2)$, the second term in the square brackets of Eq. (3.18) doubles the result, whereas for $\alpha=\beta$ and $J=$ $2 j_{\alpha} \bmod (2), V_{\alpha \beta}=0$. The factor $\mathcal{N}_{\alpha \beta}$ provides the correct normalization.

In the processes associated with the electron capture, shell electrons of the parent atom appear in a superposition of the stationary states of the daughter atom. The overlap amplitude of two atoms with atomic numbers $Z$ and $Z+\Delta Z$ can be evaluated for $\Delta Z \ll Z$ in a simple nonrelativistic shell model to give (Krivoruchenko and Tyrin, 2020)

$$
K_{Z} \approx \exp \left(-\frac{3^{5 / 3} 2^{1 / 3}}{80} \frac{\Delta Z^{2}}{Z^{1 / 3}}\right)
$$

The overlap factors for ${ }^{96} \mathrm{Ru},{ }^{152} \mathrm{Gd}$, and ${ }^{190} \mathrm{Pt}$ atoms equal $K_{Z}=0.895,0.906$, and 0.912 , respectively. The result is not sensitive to the charge. Valence-shell electrons are involved in the formation of chemical bonds and give an important contribution to $K_{Z}$. We limit ourselves to estimating the core-shell electron contribution that weakly depends on the environment.

The weak charged current of a nucleus for a low-energy transfer can be written in the form

$$
J^{\mu}(0, \mathbf{r})=\sum_{a} \tau_{a}^{-}\left[g_{V} g^{\mu 0}+g_{A}\left(\sigma_{k}\right)_{a} g^{\mu k}\right] \delta\left(\mathbf{r}-\mathbf{r}_{a}\right)
$$

Equation (3.20) neglects the contribution of the exchange currents. The short-term contribution of some higherdimensional operators is dominated by the pion exchange mechanism; see Faessler et al. (2008).

The neutrino momentum enters the energy denominators of Eq. (3.18). The typical value of $q_{0}$ is of the order of the Fermi momentum $p_{F}=270 \mathrm{MeV}$. The remaining quantities in the energy denominators are of the order of the nucleon binding energy in the nucleus $\sim 8 \mathrm{MeV}$, i.e., substantially lower. The energy denominators can therefore be taken out of the square brackets such that the sum over the excited states can be performed using the completeness condition $\sum_{n}|n\rangle\langle n|=1$. This approximation is called the closure approximation. The integral over q entering Eq. (3.18) with good accuracy is inversely proportional to the distance between two nucleons. The decay amplitude can finally be written in the form [see Krivoruchenko et al. (2011)]

$V_{\alpha \beta} \approx G_{F}^{2} \cos ^{2} \theta_{C} K_{Z} m_{\beta \beta} \frac{g_{A}^{2}}{4 \pi R} \sqrt{2 J_{f}+1} M^{2 \mathrm{EC}} \mathcal{A}_{\alpha \beta}$

Here the electron and nuclear parts of the amplitude are assumed to factorize. Such an approximation is well justified in the case of $K$ capture given the approximate constancy of the electron wave functions inside the nucleus. The stillprobable capture of an electron from the $p_{1 / 2}$ state is determined by the lower dominant component of the electron wave function inside the nucleus, which is also approximately constant. The factorization is also supported by the fact of localization of nucleons involved in the decay near the nuclear surface.

The decay amplitude due to the operators of higher dimension of Figs. 3(b) and 3(c) has the form of Eq. (3.21) with the replacement

$$
\begin{aligned}
& m_{\beta \beta} \frac{g_{A}^{2}}{4 \pi R} \sqrt{2 J_{f}+1} M^{2 \mathrm{EC}} \\
& \rightarrow \sum_{i=1}^{3} \beta_{i}^{X}\left(\mu_{0}, \Lambda\right) C_{i}^{X}(\Lambda)+\sum_{i=1}^{5} \beta_{i}^{X Y}\left(\mu_{0}, \Lambda\right) C_{i}^{X Y}(\Lambda) .
\end{aligned}
$$

The value of $\mathcal{A}_{\alpha \beta}$ entering Eq. (3.21) is the product of electron wave functions, whose bispinor indices are contracted in a way depending on the type of nuclear transition and the type of operator responsible for the decay.

For the neutrino exchange mechanism, the explicit expressions for $\mathcal{A}_{\alpha \beta}$ of low- $J$ nuclear transitions $0^{+} \rightarrow 0^{ \pm}, 1^{ \pm}$in terms of the upper and lower radial components of the electron wave functions were given by Krivoruchenko et al. (2011). For $j_{\alpha}=j_{\beta}=1 / 2$ and arbitrary $n_{\alpha}$ and $n_{\beta}$, one gets

$$
\begin{gathered}
\mathcal{A}_{\alpha \beta}\left(0^{+} \rightarrow 0^{+}\right)=\left\langle F_{\alpha \beta}^{(+)}\left(r_{a}, r_{b}\right)\right\rangle \\
\mathcal{A}_{\alpha \beta}\left(0^{+} \rightarrow 0^{-}\right)=\left\langle H_{\alpha \beta}^{(+)}\left(r_{a}, r_{b}\right)\right\rangle \\
\mathcal{A}_{\alpha \beta}\left(0^{+} \rightarrow 1^{+}\right) \approx\left\langle F_{\alpha \beta}^{(-) 2}\left(r_{a}, r_{b}\right)\right\rangle^{1 / 2} \\
\mathcal{A}_{\alpha \beta}\left(0^{+} \rightarrow 1^{-}\right) \approx\left\langle\left[H_{\alpha \beta}^{(-)}\left(r_{a}, r_{b}\right)-H_{\alpha \beta}^{(-)}\left(r_{b}, r_{a}\right)\right]^{2} / 4\right\rangle^{1 / 2}
\end{gathered}
$$

The functions $F^{( \pm)}$and $H^{( \pm)}$depend on the radial variables $r_{a}$ and $r_{b}$ and quantum numbers $\alpha$ and $\beta$ of the captured electrons. For $l_{\alpha}=l_{\beta}=0$, one finds that $4 \pi F_{\alpha \beta}^{( \pm)}\left(r_{a}, r_{b}\right)=$ $\mathcal{N}_{\alpha \beta}\left[f_{\alpha}\left(r_{a}\right) f_{\beta}\left(r_{b}\right) \pm f_{\beta}\left(r_{a}\right) f_{\alpha}\left(r_{b}\right)\right] \quad$ and $4 \pi H_{\alpha \beta}^{( \pm)}\left(r_{a}, r_{b}\right)=$ $\mathcal{N}_{\alpha \beta}\left[g_{\alpha}\left(r_{a}\right) f_{\beta}\left(r_{b}\right) \pm g_{\beta}\left(r_{a}\right) f_{\alpha}\left(r_{b}\right)\right]$. Computation of electron radial wave functions $f_{\alpha}(r)$ and $g_{\alpha}(r)$ is discussed in Sec. IV. Nuclear-structure models for matrix elements $M^{2 \mathrm{EC}}$ entering Eq. (3.21) are discussed in Sec. V.

\section{Comparison of $0 \nu 2 \mathrm{EC}$ and $0 \nu 2 \beta^{-}$decay half-lives}

Here we obtain estimates for half-lives of the $0 \nu 2 \mathrm{EC}$ and $0 \nu 2 \beta^{-}$decay, starting from the expressions by Suhonen and Civitarese (1998). The inverse $0 \nu 2 \beta^{-}$half-life can be written in the form

$$
\left(T_{1 / 2}^{0 \nu 2 \beta}\right)^{-1}=\left(\frac{\left|m_{\beta \beta}\right|}{m}\right)^{2}\left|M_{2 \beta}\right|^{2} G_{2 \beta},
$$

where $M_{2 \beta}$ is the nuclear matrix element of the $0 \nu 2 \beta^{-}$decay, $m$ is the electron rest mass, $G_{2 \beta}=g_{\mathrm{A}}^{4} K_{Z}^{2} g^{(0 \nu)} r_{A}^{-2} \mathcal{I}$ is the phasespace factor, with $r_{A}=m R, R=1.2 A^{1 / 3}$ fm the nuclear radius. $K_{Z}$ describes the overlap of the electron shells of the parent and daughter atoms, including the possible ionization of the latter. In what follows, we neglect the electron shell 
effects and set $K_{Z}=1$. The factor $g^{(0 \nu)}=2.80 \times 10^{-22} \mathrm{yr}^{-1}$ includes all the fundamental constants and other numerical coefficients entering the half-life. The phase-space integral reads

$$
\mathcal{I}=\int_{1}^{\tilde{Q}+1} F_{0}\left(Z_{f}, \varepsilon_{1}\right) F_{0}\left(Z_{f}, \varepsilon_{2}\right) p_{1} p_{2} \varepsilon_{1} \varepsilon_{2} d \varepsilon_{1}
$$

where $\varepsilon_{1,2}$ are the total energies and $p_{1,2}$ are the momenta of the emitted electrons, scaled by the electron mass. Here $\tilde{Q}=Q / m$ is the normalized $Q$ value of the decay. The quantities $F_{0}\left(Z_{f}, \varepsilon\right)$ are the Fermi functions taking into account the Coulomb interaction between the emitted electrons and the final nucleus with charge number $Z_{f}$. The integral $\mathcal{I}$ can be integrated analytically by noticing that $\varepsilon_{2}=\tilde{Q}+2-\varepsilon_{1}$ and using the Primakoff-Rosen approximation $F_{0}\left(Z_{f}, \varepsilon\right)=(\varepsilon / p) F_{0}^{(\mathrm{PR})}\left(Z_{f}\right)$. This leads in a good approximation to $\mathcal{I} \approx 10 \pi^{2} \alpha^{2} Z_{f}^{2}(\tilde{Q}+1)^{5} / 3$ [see Suhonen and Civitarese (1998)] and to the corresponding phase-space factor $G_{2 \beta}=g_{\mathrm{A}}^{4} Z_{f}^{2} A^{-2 / 3}(\tilde{Q}+1)^{5} 5 \times 10^{-20} \mathrm{yr}^{-1}$. Combining with the rest of the $0 \nu 2 \beta$ observables, the inverse half-life can be written as

$$
\begin{aligned}
& \left(T_{1 / 2}^{0 \nu 2 \beta}\right)^{-1} \\
& =g_{\mathrm{A}}^{4}\left(\frac{\left|m_{\beta \beta}\right|}{m}\right)^{2}\left|M_{2 \beta}\right|^{2} Z_{f}^{2} A^{-2 / 3}(\tilde{Q}+1)^{5} 5.0 \times 10^{-20} \mathrm{yr}^{-1} .
\end{aligned}
$$

The inverse $0 \nu 2 \mathrm{EC}$ half-life is given by

$$
\left(T_{1 / 2}^{0 \nu 2 \mathrm{EC}}\right)^{-1}=\Gamma_{i} / \ln 2 \equiv G_{2 \mathrm{EC}} R_{f}
$$

where $G_{2 \mathrm{EC}}=V_{\alpha \beta}^{2} / \ln 2$ and $R_{f}$ is defined by Eq. (3.6). For $J_{f}^{\pi}=0^{+}$and $K_{Z}=1$, we find in the nonrelativistic approximation for two-electron capture from the lowest $K$ shell

$$
\begin{aligned}
& \left(T_{1 / 2}^{0 \nu 2 \mathrm{EC}}\right)^{-1} \\
& \quad=g_{\mathrm{A}}^{4}\left(\frac{\left|m_{\beta \beta}\right|}{m}\right)^{2}\left|M_{2 \mathrm{EC}}\right|^{2} Z_{i}^{6} A^{-2 / 3} \alpha^{2} m R_{f} 5.1 \times 10^{-25} \mathrm{yr}^{-1},
\end{aligned}
$$

where $M_{2 \mathrm{EC}}$ is the $0 \nu 2 \mathrm{EC}$ nuclear matrix element.

We can now find the ratio of the two processes. Adopting the simplification $Z_{f} \approx Z_{i} \equiv Z$ and assuming that $M_{2 \beta} \approx M_{2 \mathrm{EC}}$, one finds for the half-life ratio

$$
\frac{T_{1 / 2}^{0 \nu 2 \mathrm{EC}}}{T_{1 / 2}^{0 \nu 2 \beta}} \approx\left(\frac{20}{Z}\right)^{4} \frac{(\tilde{Q}+1)^{5}}{\alpha^{2} m R_{f}}
$$

Given that $\Gamma_{f} \sim \alpha^{2} m=27.2 \mathrm{eV}$, one immediately derives that the two processes have comparable half-lives for $\alpha^{2} m R_{f} \sim 1$, which is the case for $\left|M_{i}-M_{f}\right| \lesssim \Gamma_{f}$.

\section{ELECTRON SHELL EFFECTS}

The selection of atoms with near-resonant $0 \nu 2 \mathrm{EC}$ transitions requires an accurate value of the double-electron ionization potentials of the atoms and the electron wave functions in the nuclei. The electron shell models are based on the Hartree-Fock and post-Hartree-Fock methods, density functional theory, and semiempirical methods of quantum chemistry. Analytical parametrizations of the nonrelativistic wave functions of electrons in neutral atoms, obtained with the use of the Roothaan-Hartree-Fock method and covering almost the entire periodic table, were provided by Clementi and Roetti (1974), McLean and McLean (1981), Snijders, Vernooijs, and Baerends (1981), and Bunge, Barrientos, and Bunge (1993). The various feasible 0 $22 \mathrm{EC}$ decays are expected to occur in medium-heavy and heavy atoms, for which relativistic effects are important. With the advent of personal computers, physicists acquired the opportunity to use advanced software packages, such as GRASP2K (Dyall et al., 1989; Grant, 2007), DIRAC, ${ }^{1}$ CI-MBPT (Kozlov et al., 2015), and others, for applications of relativistic computational methods in modeling complex atomic systems.

Quantum electrodynamics (QED) of electrons and photons is known to be a self-consistent theory within infinite renormalizations. One could expect the existence, at least, of a similarly formally consistent theory of electrons, photons, and nuclei, regarded as elementary particles, which would be a satisfactory idealization for most practical purposes.

In quantum field theory, the relativistic bound states of two particles are described by the Bethe-Salpeter equation (Salpeter and Bethe, 1951; Hayashi and Munakata, 1952). In the nonrelativistic limit, this equation leads to the Schrödinger wave equation, but it also includes additional anomalous solutions that do not have a clear physical interpretation: First, there are bound states corresponding to excitations of the timelike component of the relative four momenta of the particles. Such states have no analogs in the nonrelativistic potential scattering theory. None of the particles observed experimentally have been identified with the anomalous solutions thus far. Second, some solutions appear with a negative norm. Third, the Bethe-Salpeter kernel, evaluated at any finite order of perturbation theory, breaks crossing symmetry and gauge invariance of QED (Nakanishi, 1969; Itzykson and Zuber, 1980). The anomalous solutions do not arise when retardation effects are neglected.

Applications of the Bethe-Salpeter equation to the hydrogen atom (Salpeter, 1952) and positronium (Itzykson and Zuber, 1980) appeared to be successful because of the nonrelativistic character of the bound-state problems and the possibility to account for the retardation effects with the help of perturbation theory in terms of the small parameter $1 / c$.

A successful attempt at generalization of the series expansion around the instantaneous approximation to multielectron

\footnotetext{
${ }^{1}$ See T. Saue, L. Visscher, H. J. Aa. Jensen, and R. Bast, with contributions from V. Bakken et al. (2018), "DIRAC, a relativistic $a b$ initio electronic structure program, release DIRAC18 (2018)" (available at https://doi.org/10.5281/zenodo.2253986; see also http://www.diracprogram.org).
} 
atoms was presented by Sucher (1980) and Broyles (1988), where the progress was achieved by using noncovariant perturbation theory in the coupling constant $\alpha$ of electrons with the transverse part of the electromagnetic vector potential and the magnitude of $Z$ diagrams describing creation and annihilation of electron-positron pairs. Such a perturbation theory appears to be well founded because the transverse components of the electromagnetic vector potential interact with spatial components of the electromagnetic current, which contain the small term $1 / c$, whereas $Z$ diagrams contribute to observables in higher orders of $1 / c$. Compared to lowest-order Coulomb photon exchange diagrams, $Z$ diagrams are suppressed by the factors $u^{\dagger} v^{\prime} \sim 1 / c$ and $v^{\prime \dagger} u \sim 1 / c$ in each of the photon vertices due to overlapping of the small and large bispinor components and the factor $1 / c^{2}$ originating from the propagator of the positron. As a result, $Z$ diagrams are of the order of $O\left(\alpha^{2} / c^{4}\right)$. The second-order correction to the energy due to the Fermi-Breit potential is of the same magnitude. The exchange of transverse photons leading to the FermiBreit potential contributes to the interaction potential of the order of $\alpha / c^{2}$, such that the magnitude of the $Z$ diagrams is suppressed. The scheme adopted by Sucher (1980) and Broyles (1988) is equivalent to neglecting the Dirac sea at the zeroth-order approximation, except for the two-body problem, in which the bound-state energy equation is the same as given by Salpeter (1952). The no-sea approximation is widely used to study nuclear matter in the Dirac-BruecknerHartree-Fock method (Anastasio et al., 1983; Brockmann and Machleidt, 1984; ter Haar and Malfliet, 1987). We remark that the negative-energy fermion states are required to ensure causality and guarantee Lorentz invariance of the $T$ product and hence of the $S$ matrix.

The self-consistent nonrelativistic expansion becomes possible because the corrections related to the finite speed of light are small. The neglect of retardation allows for the formulation of an equation for the Bethe-Salpeter wave function integrated over the relative energy of the particles (Broyles, 1988). The noncovariant wave function obtained in this manner yields the wave function of the equivalent many-body noncovariant Schrödinger equation. Gauge invariance of QED ensures the Lorentz invariance of the theory, but in the intermediate stages of the computation it is necessary to work with Lorentz-noncovariant and gaugedependent expressions.

In the Feynman gauge, the product of the two photon vertices and the photon propagator

$$
D_{\mu \nu}(k)=\frac{-g_{\mu \nu}}{k^{2}}
$$

is represented as follows:

$$
e \gamma_{1}^{\mu} D_{\mu \nu}(k) e \gamma_{2}^{\nu}=-\gamma_{1}^{0} \gamma_{2} \frac{e^{2}}{k^{2}}\left(1-\boldsymbol{\alpha}_{1} \boldsymbol{\alpha}_{2}\right),
$$

where $k=(\omega, \mathbf{k})$ is the photon momentum, $\boldsymbol{\alpha}_{\mathbf{i}}=\boldsymbol{\gamma}_{\mathbf{i}}^{0} \boldsymbol{\gamma}_{\mathbf{i}}$ are velocity operators for the particles $\mathbf{i}=1,2$, and $\gamma^{\mu}$ are the Dirac $\gamma$ matrices. The corresponding interaction potential obtained in the static limit

$$
\begin{aligned}
V_{\mathrm{CG}}(\mathbf{r}) & \equiv \int \frac{d \mathbf{k}}{(2 \pi)^{3}} \exp (i \mathbf{k} \cdot \mathbf{r}) e \gamma_{1}^{0} \gamma_{1}^{\mu} D_{\mu \nu}(\omega=0, \mathbf{k}) e \gamma_{2}^{0} \gamma_{2}^{\nu} \\
& =\frac{e^{2}}{4 \pi r}\left(1-\boldsymbol{\alpha}_{1} \boldsymbol{\alpha}_{2}\right)
\end{aligned}
$$

acquires a familiar form of electrostatic interaction energy of charges plus magnetostatic interaction energy of electric currents. The correction to the Coulomb potential entering Eq. (4.3) was first derived quantum mechanically by Gaunt (1929). The expansion of $D_{\mu \nu}(k)$ in higher powers of $\omega$ describes retardation effects, which are expected to be most pronounced for inner orbits. A typical splitting of the energy levels is $\omega \sim \alpha^{2} Z^{2} m$, and a typical momentum of electrons is $|\mathbf{k}| \sim \alpha Z m$, such that for light and medium-heavy atoms the expansion parameter $\omega^{2} / \mathbf{k}^{2} \sim(\alpha Z)^{2}$ is still sufficiently small. The potential of Eq. (4.3) is known as the Coulomb-Gaunt potential. Such a potential can be used to approximate the lowest-order interaction of electrons, although the magnetostatic energy $\sim 1 / c^{2}$ is of the same order as the retardation corrections to the Coulomb potential.

In the Coulomb gauge, the photon propagator $D_{\mu \nu}(k)$ takes the form

$$
\begin{aligned}
D_{00}(k) & =\frac{1}{\mathbf{k}^{2}}, \\
D_{i j}(k) & =\frac{\delta_{i j}-k_{i} k_{j} / \mathbf{k}^{2}}{k^{2}}, \\
D_{i 0}(k) & =D_{0 j}(k)=0,
\end{aligned}
$$

where $i, j=1,2,3$. The Coulomb gauge breaks Lorentz covariance but appears natural in the problem of quantization of the electromagnetic field, since it allows for explicitly solving the constraint equations. The photon propagator appears split in two pieces, the first of which corresponds to the instantaneous interaction; the second describes the static terms $\sim 1 / c^{2}$ plus retardation effects $\sim 1 / c^{4}$. The potential of a zeroth-order approximation contains contributions from the timelike components and the spacelike component of the propagator in the limit of $\omega=0$. The product of the two photon vertices and the propagator (4.4) is represented by

$$
e \gamma_{1}^{\mu} D_{\mu \nu}(k) e \gamma_{2}^{\nu}=\gamma_{1}^{0} \gamma_{2}^{0}\left[\frac{e^{2}}{\mathbf{k}^{2}}+\frac{e^{2}}{k^{2}}\left(\boldsymbol{\alpha}_{1} \boldsymbol{\alpha}_{2}-\frac{\alpha_{1} \mathbf{k} \alpha_{2} \cdot \mathbf{k}}{\mathbf{k}^{2}}\right)\right] .
$$

The interaction potential corresponding to the static limit of Eq. (4.5) becomes

$$
V_{\mathrm{CB}}(\mathbf{r})=\frac{e^{2}}{4 \pi r}\left(1-\frac{\boldsymbol{\alpha}_{\mathbf{1}} \boldsymbol{\alpha}_{2}+\alpha_{1} \mathbf{n} \alpha_{\mathbf{2}} \cdot \mathbf{n}}{2}\right),
$$

where $\mathbf{n}=\mathbf{r} /|\mathbf{r}|$. Equation (4.6) can be recognized as the sum of the classical Coulomb and Darwin potentials (Darwin, 1920), which demonstrates the essentially classical origin of $V_{\mathrm{CB}}$. The no-sea approximation is thus sufficient to ensure the correct expression for $V_{\mathrm{CB}}$. The potential $V_{\mathrm{CB}}$ given by Eq. (4.6) was first derived quantum mechanically by Breit (1929); it is known as the Coulomb-Breit potential. Starting 
with $V_{\mathrm{CB}}$ is a natural choice, since the retardation effects in this case are of the order of $1 / c^{4}$.

The techniques of Sucher (1980) and Broyles (1988) can be considered as the starting point for developing a systematic $1 / c$ expansion around the instantaneous approximation in the bound-state problem for light and medium-heavy atoms in analogy with positronium and the hydrogen atom. Heavy atoms for which the expansion parameter $1 / c$ is not small are somewhat beyond the scope of perturbational treatment. Since theoretical estimates of accuracy are difficult, it is required to compare model predictions with empirical data wherever possible.

The relativistic approach is based on the Dirac-Coulomb Hamiltonian

$$
H=\sum_{i}\left(\alpha \mathbf{p}_{i}+\beta m-\frac{\alpha Z}{r_{i}}\right)+\sum_{i<j} V\left(\mathbf{r}_{i}-\mathbf{r}_{j}\right)
$$

where the sum runs over electrons $r_{i}=\left|\mathbf{r}_{i}\right|$. The potential $V(\mathbf{r})$ is, as a rule, taken to be the Coulomb-Breit potential, which already accounts for relativistic effects $\sim 1 / c^{2}$ at the lowest order of the perturbation expansion. Neglecting the Dirac sea could require the projection of the potential onto positive energy states. The electron wave function is constructed as a Slater determinant of one-electron orbitals. Solutions to the eigenvalue problem are sought using the Dirac-Hartree-Fock approximation.

In the nonrelativistic Coulomb problem, physical quantities are determined by a single parameter, which is the Bohr radius $a_{B}=1 / \alpha m=5.2 \times 10^{4} \mathrm{fm}$. In the relativistic problem, the Bohr radius acts as a scale, which determines the normalization of electron wave functions and the integral characteristics, such as the interaction energies of holes in the electron shell. In addition to the Bohr radius, there are other scales of the relativistic problem. The electron Compton wavelength $\lambda_{e}=$ $1 / m$ determines the distance from the nucleus at which the electron should be considered in a relativistic manner. On a scale smaller than $1 / m$, the nonrelativistic wave function is markedly different than the upper component of the Dirac wave function; thus, the effects associated with the finite size of the nucleus must be calculated on the basis of the relativistic Dirac equation. The third scale in the hierarchy is the distance $\alpha Z / m=2.8 Z$ fm at which the Coulomb potential becomes greater than the electron mass. The smallest (fourth) scale is the nuclear radius $R=1.2 A^{1 / 3} \mathrm{fm}$. The size of the ${ }^{238} \mathrm{U}$ nucleus is approximately 35 times smaller than $\alpha Z / m=260 \mathrm{fm}$, approximately 50 times smaller than the Compton wavelength $1 / m=390 \mathrm{fm}$, and approximately 75 times smaller than $1 / \alpha m Z=580 \mathrm{fm}$. The depth of the potential extending from 0 to $\alpha Z / m$ is too small to produce bound states of electrons in the negative continuum.

According to the Thomas-Fermi model, the majority of the shell electrons are at a distance $1 / \alpha m Z^{1 / 3}$ from the nucleus, and the total binding energy of the electrons scales as $20.8 Z^{7 / 3} \mathrm{eV}$. The potential energy of the interaction of electrons with each other is $1 / 7$ of the energy of the interaction of electrons with the nucleus. The numerical smallness of the electron-electron interaction shows that the
Coulomb wave functions of electrons can be used as a first approximation to the self-consistent mean-field solutions.

The single-electron ionization potentials (SEIPs) of innermost orbits, which are of specific interest to the $0 \nu 2 \mathrm{EC}$ problem, increase quadratically with $Z$ from $13.6 \mathrm{eV}$ in the hydrogen atom up to $115.6 \mathrm{keV}$ in the uranium atom. The radii of the outer orbits and hence the size of the atoms do not depend on $Z$. The SEIPs of outermost orbits are of the order of a few $\mathrm{eV}$ for all $Z$. The greatest overlap with the nucleus is achieved for electrons of the innermost orbits. In the $(A, Z) \rightarrow$ $(A, Z-2)$ transitions associated with the $0 \nu 2 \mathrm{EC}$ decays, we are interested in the electron wave functions inside the parent nucleus $(A, Z)$, whereas the energy balance is provided by the energy of the excited electron shells of the daughter nucleus $(A, Z-2)$. The SEIPs of all orbitals across the entire periodic table were given by Larkins (1977), where experimental data on the binding energy of electron subshells and data obtained from Hartree-Fock atomic calculations were combined within a general semiempirical method.

The double-electron ionization potentials (DEIPs) are additive to first approximation. A more accurate estimate of DEIPs takes into account the interaction energy of electron holes, relaxation energy, and other specific effects. In the innermost orbitals, the Coulomb interaction energy of two holes is of the order of $\alpha Z / a_{B} \sim \alpha^{2} Z m$. This energy grows linearly with $Z$ and reaches a value of $\sim 1 \mathrm{keV}$ in heavy atoms. The relaxation energy for a medium-heavy atom of ${ }^{101} \mathrm{Ru}$ reaches a value of $400 \mathrm{eV}$ (Niskanen et al., 2011). The twohole excitation energy of the daughter atoms differs from the corresponding DEIP by the sum of the energies of two outermost occupied orbits, approximately $10 \mathrm{eV}$.

The required accuracy of two-hole excitation energies is dictated by the typical width of vacancies of electron shells, which is approximately $10 \mathrm{eV}$. This accuracy is required to specify the $0 \nu 2 \mathrm{EC}$ transitions in the unitary limit. The best achieved accuracy in the $Q$-value measurements with Penning traps is of the order of $10 \mathrm{eV}$ for heavy systems, and furthermore the DEIP calculations for heavy atoms are successful to within several tens of $\mathrm{eV}$. To realistically calculate the excitation energies and the short-distance components of electron wave functions, we use the GRASP2K software package (Dyall et al., 1989; Grant, 2007), which is well founded theoretically and successful in the description of a wide range of atomic physics data.

\section{A. Interaction energy of electron holes}

The average electron velocity $v / c \sim \alpha Z / n$ increases with the nuclear charge and becomes large in heavy atoms. In a uranium atom, an electron on the $K$ shell, localized at an average distance $a_{B} / Z \sim 600 \mathrm{fm}$ from the nucleus, moves at a speed of $v \sim 0.7 c$. A fully relativistic description is thus required to construct accurate electron wave functions inside the nucleus.

The wave function of a relativistic electron in a central potential is defined in Eq. (3.13). We consider transitions between nuclei with good quantum numbers. In what follows, $J_{i}$ and $J_{f}$ are the total angular momenta of the parent and daughter nuclei and $j_{i}$ and $j_{f}$ are the total angular momenta of 
electron shells in the initial and final states, respectively. The daughter nucleus $(A, Z-2)$ inherits the electron shell of the parent nucleus $(A, Z)$ with two electron holes formed by the electron capture and possible excitations of spectator electrons into vacant orbits. The total angular momentum of the captured electrons $J$ is in the interval $\max \left(\left|J_{f}-J_{i}\right|\right.$, $\left.\left|j_{f}-j_{i}\right|\right) \leq J \leq \min \left(J_{f}+J_{i}, j_{f}+j_{i}\right)$.

Let $\mathbf{J}_{i}^{\text {tot }}=\mathbf{J}_{i}+\mathbf{j}_{i}$ and $\mathbf{J}_{f}^{\text {tot }}=\mathbf{J}_{f}+\mathbf{j}_{f}$ be the total angular momenta of atoms in the initial and final states, respectively. The reaction involves the nucleus and two electrons, whereas $Z-2$ electrons are spectators that can be excited due to the nuclear recoil or the nonorthogonality of the initial- and finalstate electron wave functions. Total angular-momentum conservation implies that $\mathbf{J}_{f}^{\text {tot }}=\mathbf{J}_{i}^{\text {tot }}$ as well as $\mathbf{J}_{f}=\mathbf{J}_{i}+\mathbf{J}$ and $\mathbf{j}_{f}+\mathbf{J}=\mathbf{j}_{i}$.

The atomic-state wave function with definite $j_{i}$ is a superposition of configuration states, which are antisymmetric products of the one-electron orbitals (3.13). For $J_{i}^{\text {tot }} \neq 0$, the atomic-state wave function is further superimposed with the wave function of the nucleus. The atomic states are split into levels with typical energy separation of fractions of electron volts. Transitions between these levels produce radiation in the short-wavelength and midwavelength infrared ranges. Such effects lie beyond the energy scale in which we are interested $10 \mathrm{eV}$. Since at room temperature atoms are in their ground states, each time we select the lowest-energy eigenstate. In the $0 \nu 2 \mathrm{EC}$ decays, the spin of the initial nucleus is zero, in which case the configuration space reduces and the calculations simplify.

The capture from the $s_{1 / 2}$ and $p_{1 / 2}$ orbits occurs with the dominant probability, which restricts the admissible values of $J^{\pi}$ to $0^{ \pm}, 1^{ \pm}$. The higher orbits, relevant to the daughter nuclei with $J_{f} \geq 2$, thus may be disregarded. The spin $J$ is the suitable quantum number for the classification of transitions.

After capturing the pair, the atomic-state wave function is still a superposition of configuration states, onto which the states with various $j_{f}$ are further superimposed. The typical level splitting is a fraction of an electron volt, whereas the radiation width of the excited electron shell is about $10 \mathrm{eV}$. This is the case for overlapping resonances. The influence of the coherent overlap on the $0 \nu 2 \mathrm{EC}$ decay has not been discussed thus far. We sum all the contributions decoherently.

The two-electron wave function has the total angular momentum $J$, projection $M$, and a definite parity. This can be arranged by weighting the product of wave functions of one-electron orbitals (3.13) with the Clebsch-Gordan coefficients, as done in Eq. (3.15). The Pauli principle says that the wave function must be antisymmetric under an exchange of two electrons. The normalized antisymmetric two-electron wave function takes the form shown in Eq. (3.16). The interaction energy of electron holes in the static approximation can be found from

$$
\epsilon=\int d \mathbf{r}_{1} d \mathbf{r}_{2} \Psi_{\beta \delta}^{J M^{\dagger}}\left(\mathbf{r}_{1}, \mathbf{r}_{2}\right) V\left(\mathbf{r}_{1}-\mathbf{r}_{2}\right) \Psi_{\beta \delta}^{J M}\left(\mathbf{r}_{1}, \mathbf{r}_{2}\right),
$$

where $V(\mathbf{r})$ is the Coulomb-Gaunt potential (4.3) or the Coulomb-Breit potential (4.6).
The interaction energy (4.8) is given by the matrix element of the two-particle operator. In such cases, the angular variables are explicitly integrated out and the problem is reduced to the calculation of a two-dimensional integral in the radial variables; see Grant (2007). We present results of this reduction needed to demonstrate the independence of the interaction energy from the gauge.

\section{Electrostatic interaction}

The interaction energy in the static approximation splits into the sum of the electrostatic and magnetostatic energies $\epsilon=\epsilon_{E}+\epsilon_{M}$. The Coulomb part $\epsilon_{E}$ does not depend on the gauge condition. Equation (4.8) can be written in the form

$$
\begin{aligned}
\epsilon_{E} & =\int d \mathbf{r}_{1} d \mathbf{r}_{2} \Psi_{\beta \delta}^{J M \dagger}\left(\mathbf{r}_{1}, \mathbf{r}_{2}\right) \frac{\alpha}{r} \Psi_{\beta \delta}^{J M}\left(\mathbf{r}_{1}, \mathbf{r}_{2}\right) \\
& =2 \mathcal{N}_{\beta \delta}^{2}\left[\mathcal{K}_{\beta \delta \beta \delta}^{J M}-(-)^{j_{\beta}+j_{\delta}-J} \mathcal{K}_{\beta \delta \delta \beta}^{J M}\right]
\end{aligned}
$$

where $4 \pi \alpha=e^{2}, r=\left|\mathbf{r}_{1}-\mathbf{r}_{2}\right|$, and

$$
\mathcal{K}_{\alpha \gamma \beta \delta}^{J M}=\sum_{m_{\alpha} m_{\gamma} m_{\beta} m_{\delta}} C_{j_{\alpha} m_{\alpha} j_{\gamma} m_{\gamma}}^{J M} C_{j_{\beta} m_{\beta} j_{\delta} m_{\delta}}^{J M} \mathcal{K}_{\beta m_{\beta} \delta m_{\delta}}^{\alpha m_{\alpha} \gamma m_{\gamma}},
$$

with

$$
\mathcal{K}_{\beta m_{\beta} \delta m_{\delta}}^{\alpha m_{\gamma} \gamma m_{\gamma}}=\int d \mathbf{r}_{1} d \mathbf{r}_{2} \Psi_{\alpha m_{\alpha}}^{\dagger}\left(\mathbf{r}_{1}\right) \Psi_{\beta m_{\beta}}\left(\mathbf{r}_{1}\right) \frac{\alpha}{r} \Psi_{\gamma m_{\gamma}}^{\dagger}\left(\mathbf{r}_{2}\right) \Psi_{\delta m_{\delta}}\left(\mathbf{r}_{2}\right) .
$$

The Hermitian product of spherical spinors weighted with a spherical harmonic and integrated over angles can be represented as

$$
\int d \Omega_{\mathbf{n}} \Omega_{\alpha m_{\alpha}}^{\dagger}(\mathbf{n}) \Omega_{\beta m_{\beta}}(\mathbf{n}) Y_{l m}(\mathbf{n})=C_{j_{\beta} m_{\beta} l m}^{j_{\alpha} m_{\alpha}} \mathcal{A}_{\alpha \beta}^{l},
$$

where $\left|j_{\alpha}-j_{\beta}\right| \leq l \leq j_{\alpha}+j_{\beta}$,

$\mathcal{A}_{\alpha \beta}^{l}=(-)^{1 / 2+j_{\beta}+l_{\alpha}+l} \sqrt{\frac{[l]\left[l_{\beta}\right]\left[j_{\beta}\right]}{4 \pi}} C_{l_{\beta} 0 l 0}^{l_{\alpha} 0}\left\{\begin{array}{lll}1 / 2 & l_{\beta} & j_{\beta} \\ l & j_{\alpha} & l_{\alpha}\end{array}\right\}$,

and $[x]=2 x+1$. By introducing the unit matrix $(\boldsymbol{\sigma n})^{2}=1$ between the spherical spinors of Eq. (4.11) and taking into account the identity $\boldsymbol{\sigma n} \Omega_{\alpha m_{\alpha}}(\mathbf{n})=-\Omega_{\alpha^{\prime} m_{\alpha}}(\mathbf{n})$, one obtains $\mathcal{A}_{\alpha \beta}^{l}=\mathcal{A}_{\alpha^{\prime} \beta^{\prime}}^{l}$. The angular integral of the Hermitian product of the electron wave functions and a spherical harmonic leads to

$\int d \Omega_{\mathbf{n}} \Psi_{\alpha m_{\alpha}}^{\dagger}(\mathbf{n}) \Psi_{\beta m_{\beta}}(\mathbf{n}) Y_{l m}(\mathbf{n})=C_{j_{\beta} m_{\beta} l m}^{j_{\alpha} m_{\alpha}} \mathcal{F}_{\alpha \beta}^{l}(r)$,

where $\mathcal{F}_{\alpha \beta}^{l}(r)$ is defined by

$$
\mathcal{F}_{\alpha \beta}^{l}(r)=\left[f_{\alpha}(r) f_{\beta}(r)+g_{\alpha}(r) g_{\beta}(r)\right] \mathcal{A}_{\alpha \beta}^{l} .
$$

The electrostatic interaction integral takes the form (Krivoruchenko et al., 2011) 
$\mathcal{K}_{\alpha \gamma \beta \delta}^{J M}=\alpha \sum_{l} \frac{4 \pi}{2 l+1} \mathcal{C}_{\alpha \gamma \beta \delta}^{J l} \int r_{1}^{2} d r_{1} r_{2}^{2} d r_{2} \frac{r_{<}^{l}}{r_{>}^{l+1}} \mathcal{F}_{\alpha \beta}^{l}\left(r_{1}\right) \mathcal{F}_{\gamma \delta}^{l}\left(r_{2}\right)$,

where $r_{i}=\left|\mathbf{r}_{i}\right|, r_{<}$is the lesser and $r_{>}$is the greater of $r_{1}$ and $r_{2}$, and

$$
\mathcal{C}_{\alpha \gamma \beta \delta}^{J l}=(-1)^{j_{\gamma}+j_{\beta}+J} \sqrt{\left[j_{\alpha}\right]\left[j_{\gamma}\right]}\left\{\begin{array}{ccc}
l & j_{\delta} & j_{\gamma} \\
J & j_{\alpha} & j_{\beta}
\end{array}\right\} .
$$

The interaction energy is invariant under rotations; thus, $\mathcal{K}_{\alpha \gamma \beta \delta}^{J M}$ does not depend on the spin projection $M$.

\section{Retardation correction in the Feynman gauge}

The timelike component of the free photon propagator in the Feynman gauge, expanded in powers of the small parameter $\omega^{2} / \mathbf{k}^{2} \sim(\alpha Z)^{2} \sim 1 / c^{2}$, takes the form

$$
D_{00}(\omega, \mathbf{k})=\frac{1}{\mathbf{k}^{2}}+\frac{\omega^{2}}{\mathbf{k}^{4}}+\cdots
$$

The second term provides the lowest-order retardation correction to the Coulomb interaction energy of electrons

$\Delta \epsilon_{E}=-\alpha \int d \mathbf{r}_{1} d \mathbf{r}_{2} \Psi_{\beta \delta}^{J M^{\dagger}}\left(\mathbf{r}_{1}, \mathbf{r}_{2}\right) \frac{\omega^{2} r}{2} \Psi_{\beta \delta}^{J M}\left(\mathbf{r}_{1}, \mathbf{r}_{2}\right)$,

where $\omega$ is the energy of virtual photon and $-r / 2$ is the Fourier transform of $4 \pi / \mathbf{k}^{4}$, obtained using the analytical continuation in $z$ of

$$
\int \frac{d \mathbf{k}}{(2 \pi)^{3}} \frac{4 \pi}{|\mathbf{k}|^{z}} e^{i \mathbf{k} \cdot \mathbf{r}}=\frac{r^{z-3} \Gamma((3-z) / 2)}{2^{z-2} \sqrt{\pi} \Gamma(z / 2)} .
$$

$\Delta \epsilon_{E}$ is of the same order as the magnetostatic interaction energy.

The angular variables can be integrated out, as in the case of the instantaneous Coulomb interaction. We write Eq. (4.18) in the form

$$
\Delta \epsilon_{E}=2 \mathcal{N}_{\beta \delta}^{2}\left[\Delta \mathcal{K}_{\beta \delta \beta \delta}^{J M}-(-)^{j_{\beta}+j_{\delta}-J} \Delta \mathcal{K}_{\beta \delta \delta \beta}^{J M}\right]
$$

where

$$
\begin{aligned}
\Delta \mathcal{K}_{\alpha \gamma \beta \delta}^{J M}= & \frac{\alpha}{2} \sum_{l} \frac{4 \pi}{2 l+1} \mathcal{C}_{\alpha \gamma \beta \delta}^{J l}\left(\epsilon_{\alpha}^{*}-\epsilon_{\beta}^{*}\right)\left(\epsilon_{\gamma}^{*}-\epsilon_{\delta}^{*}\right) \\
& \times \int r_{1}^{2} d r_{1} r_{2}^{2} d r_{2} \frac{r_{<}^{l}}{r_{>}^{l+1}}\left(\frac{r_{<}^{2}}{2 l+3}-\frac{r_{>}^{2}}{2 l-1}\right) \\
& \times \mathcal{F}_{\alpha \beta}^{l}\left(r_{1}\right) \mathcal{F}_{\gamma \delta}^{l}\left(r_{2}\right) .
\end{aligned}
$$

One can observe that only the exchange interaction $\alpha=\delta \neq$ $\gamma=\beta$ contributes to $\Delta \mathcal{K}_{\alpha \gamma \beta \delta}^{J M} \neq 0$, whereas $\Delta \mathcal{K}_{\alpha \gamma \beta \delta}^{J M}=0$ for the direct interaction $\alpha=\beta \neq \gamma=\delta$ and $\Delta \mathcal{K}_{\alpha \gamma \beta \delta}^{J M}=0$ if the electrons occupy the same shell $\alpha=\beta=\gamma=\delta$. The retardation corrections of higher orders can be calculated in a similar manner. In the Coulomb gauge, the retardation corrections to the electrostatic interaction energy vanish.

\section{Magnetostatic interaction in the Feynman gauge}

The magnetostatic part of the interaction energy (4.3) can be represented in a form similar to Eq. (4.9):

$$
\begin{aligned}
\epsilon_{M} & =-\alpha \int d \mathbf{r}_{1} d \mathbf{r}_{2} \Psi_{\beta \delta}^{J M^{\dagger}}\left(\mathbf{r}_{1}, \mathbf{r}_{2}\right) \frac{\alpha_{1} \alpha_{2}}{r} \Psi_{\beta \delta}^{J M}\left(\mathbf{r}_{1}, \mathbf{r}_{2}\right) \\
& =2 \mathcal{N}_{\beta \delta}^{2}\left[\mathcal{M}_{\beta \delta \beta \delta}^{J M}-(-)^{j_{\beta}+j_{\delta}-J} \mathcal{M}_{\beta \delta \delta \beta}^{J M}\right],
\end{aligned}
$$

where

$$
\mathcal{M}_{\alpha \gamma \beta \delta}^{J M}=\sum_{m_{\alpha} m_{\gamma} m_{\beta} m_{\delta}} C_{j_{\alpha} m_{\alpha} j_{\gamma} m_{\gamma}}^{J M} C_{j_{\beta} m_{\beta} j_{\delta} m_{\delta}}^{J M} \mathcal{M}_{\beta m_{\beta} \delta m_{\delta}}^{\alpha m_{\alpha} \gamma m_{\gamma}}
$$

with

$$
\begin{aligned}
\mathcal{M}_{\beta m_{\beta} \delta m_{\delta}}^{\alpha m_{\alpha} \gamma m_{\gamma}}= & -\int d \mathbf{r}_{1} d \mathbf{r}_{2} \Psi_{\alpha m_{\alpha}}^{\dagger}\left(\mathbf{r}_{1}\right) \alpha \Psi_{\beta m_{\beta}}\left(\mathbf{r}_{1}\right) \frac{\alpha}{r} \Psi_{\gamma m_{\gamma}}^{\dagger}\left(\mathbf{r}_{2}\right) \\
& \times \alpha \Psi_{\delta m_{\delta}}\left(\mathbf{r}_{2}\right) .
\end{aligned}
$$

The angular integrals are calculated with the use of

$\int d \Omega_{\mathbf{n}} \Omega_{\alpha m_{\alpha}}^{\dagger}(\mathbf{n}) \sigma \Omega_{\beta m_{\beta}}(\mathbf{n}) Y_{l m}(\mathbf{n})=\mathbf{e}^{\mu} \sum_{j} C_{j_{\beta} m_{\beta} j \kappa}^{j_{\alpha} m_{\alpha}} C_{1 \mu l m}^{j \kappa} \mathcal{B}_{\alpha \beta}^{j l}$,

where $\mathbf{e}^{\mu}$ are basis vectors of the cyclic coordinate system (Varshalovich, Moskalev, and Khersonskii, 1988), the sum runs over $j=l, l \pm 1$, for $\kappa=m_{\alpha}-m_{\beta}$ and $\mu=m_{\alpha}-$ $m_{\beta}-m$, and

$\mathcal{B}_{\alpha \beta}^{j l}=(-)^{j_{\alpha}-j_{\beta}+l_{\alpha}+l_{\beta}} \sqrt{\frac{3[j]\left[j_{\beta}\right][l]\left[l_{\beta}\right]}{2 \pi}} C_{l_{\beta} 0 l 0}^{l_{\alpha} 0}\left\{\begin{array}{lll}l_{\alpha} & j_{\alpha} & 1 / 2 \\ l_{\beta} & j_{\beta} & 1 / 2 \\ l & j & 1\end{array}\right\}$.

The transition current projection on a spherical harmonic can be found to be

$$
\begin{aligned}
& \int d \Omega_{\mathbf{n}} \Psi_{\alpha m_{\alpha}}^{\dagger}(\mathbf{r}) \alpha \Psi_{\beta m_{\beta}}(\mathbf{r}) Y_{l m}(\mathbf{n}) \\
&=-i \mathbf{e}^{\mu} \sum_{j} C_{j_{\beta} m_{\beta} j \kappa}^{j_{j} m_{\alpha}} C_{1 \mu l m}^{j \kappa} \mathcal{G}_{\alpha \beta}^{j l}(r),
\end{aligned}
$$

where

$$
\mathcal{G}_{\alpha \beta}^{j l}(r)=g_{\alpha}(r) f_{\beta}(r) \mathcal{B}_{\alpha^{\prime} \beta}^{j l}-f_{\alpha}(r) g_{\beta}(r) \mathcal{B}_{\alpha \beta^{\prime}}^{j l} .
$$

The sum over $j$ runs within the limits $\left|j_{\alpha}-j_{\beta}\right| \leq j \leq j_{\alpha}+j_{\beta}$, whereas $l$ are constrained by $|j-1| \leq l \leq j+1$.

The interaction integral over the radial variables takes the form 


$$
\mathcal{M}_{\alpha \gamma \beta \delta}^{J M}=-e^{2} \sum_{j l} \frac{4 \pi}{2 l+1}(-1)^{j-l} \mathcal{C}_{\alpha \gamma \beta \delta}^{J j} \int r_{1}^{2} d r_{1} r_{2}^{2} d r_{2} \frac{r_{<}^{l}}{r_{>}^{l+1}} \mathcal{G}_{\alpha \beta}^{j l}\left(r_{1}\right) \mathcal{G}_{\gamma \delta}^{j l}\left(r_{2}\right) .
$$

\section{Magnetostatic interaction in the Coulomb gauge}

In the Coulomb-Breit potential, the magnetostatic interaction energy is given by

$\epsilon_{M}^{\prime}=-\alpha \int d \mathbf{r}_{1} d \mathbf{r}_{2} \Psi_{\beta \delta}^{J M \dagger}\left(\mathbf{r}_{1}, \mathbf{r}_{2}\right) \frac{\alpha_{1} \alpha_{2}+\left(\alpha_{1} \mathbf{n}\right)\left(\alpha_{2} \mathbf{n}\right)}{2 r} \Psi_{\beta \delta}^{J M}\left(\mathbf{r}_{1}, \mathbf{r}_{2}\right)$

Using identity $n^{i} n^{j} / r=\delta^{i j} / r-\nabla^{i} \nabla^{j} r$, we integrate the derivative term by parts. The result can be written in the form $\epsilon_{M}^{\prime}=\epsilon_{M}+\Delta \epsilon_{M}$, with $\epsilon_{M}$ given by Eq. (4.21) and

$$
\Delta \epsilon_{M}=2 \mathcal{N}_{\beta \delta}^{2}\left[\Delta \mathcal{M}_{\beta \delta \beta \delta}^{J M}-(-1)^{j_{\beta}+j_{\delta}-J} \Delta \mathcal{M}_{\beta \delta \delta \beta}^{J M}\right]
$$

The variance of the magnetostatic interaction energy is determined by

$$
\Delta \mathcal{M}_{\alpha \gamma \beta \delta}^{J M}=\sum_{m_{\alpha} m_{\gamma} m_{\beta} m_{\delta}} C_{j_{\alpha} m_{\alpha} j_{\gamma} m_{\gamma}}^{J M} C_{j_{\beta} m_{\beta} j_{\delta} m_{\delta}}^{J M} \Delta \mathcal{M}_{\beta m_{\beta} \delta m_{\delta}}^{\alpha m_{\alpha} \gamma m_{\gamma}}
$$

with

$$
\begin{aligned}
& \Delta \mathcal{M}_{\beta m_{\beta} \delta m_{\delta}}^{\alpha m_{\alpha} \gamma m_{\gamma}} \\
& =-\frac{\alpha}{2} \int d \mathbf{r}_{1} d \mathbf{r}_{2}\left[\nabla \Psi_{\alpha m_{\alpha}}^{\dagger}\left(\mathbf{r}_{1}\right) \alpha \Psi_{\beta m_{\beta}}\left(\mathbf{r}_{1}\right)\right] r\left[\nabla \Psi_{\gamma m_{\gamma}}^{\dagger}\left(\mathbf{r}_{2}\right) \alpha \Psi_{\delta m_{\delta}}\left(\mathbf{r}_{2}\right)\right]
\end{aligned}
$$

The divergence of the transition current projected onto a spherical harmonic can be found to be

$$
\int d \Omega_{\mathbf{n}}\left[\nabla \Psi_{\alpha m_{\alpha}}^{\dagger}(\mathbf{r}) \alpha \Psi_{\beta m_{\beta}}(\mathbf{r})\right] Y_{l m}(\mathbf{n})=-i C_{j_{\beta} m_{\beta} l m}^{j_{\alpha} m_{\alpha}} \mathcal{H}_{\alpha \beta}^{l}(r),
$$

where

$$
\begin{aligned}
\mathcal{H}_{\alpha \beta}^{l}(r)= & {\left[-\left(\frac{d}{d r}+\frac{2-\kappa_{\alpha}+\kappa_{\beta}}{r}\right) g_{\alpha}(r) f_{\beta}(r)\right.} \\
& \left.+\left(\frac{d}{d r}+\frac{2+\kappa_{\alpha}-\kappa_{\beta}}{r}\right) f_{\alpha}(r) g_{\beta}(r)\right] \mathcal{A}_{\alpha \beta}^{l} .
\end{aligned}
$$

The variance of the magnetostatic interaction energy becomes

$$
\Delta \mathcal{M}_{\alpha \gamma \beta \delta}^{J M}=\frac{\alpha}{2} \sum_{l} \frac{4 \pi}{2 l+1} \mathcal{C}_{\alpha \gamma \beta \delta}^{J l} \times \int r_{1}^{2} d r_{1} r_{2}^{2} d r_{2} \frac{r_{<}^{l}}{r_{>}^{l+1}}\left(\frac{r_{<}^{2}}{2 l+3}-\frac{r_{>}^{2}}{2 l-1}\right) \mathcal{H}_{\alpha \beta}^{l}\left(r_{1}\right) \mathcal{H}_{\gamma \delta}^{l}\left(r_{2}\right)
$$

\section{Gauge invariance of the interaction energy of electron holes}

The wave function $\Psi_{\alpha m_{\alpha}}(\mathbf{r})$ is assumed to satisfy the Dirac equation in a mean-field potential $U(\mathbf{r})$ created by the nucleus and surrounding electrons. The divergence of the transition current between states with energies $\epsilon_{\alpha}^{*}$ and $\epsilon_{\beta}^{*}$ equals

$$
\nabla \Psi_{\alpha m_{\alpha}}^{\dagger} \alpha \Psi_{\beta m_{\beta}}=-i\left(\epsilon_{\alpha}^{*}-\epsilon_{\beta}^{*}\right) \Psi_{\alpha m_{\alpha}}^{\dagger} \Psi_{\beta m_{\beta}}
$$

We substitute this expression into Eq. (4.31). A comparison with Eq. (4.13) gives

$$
\mathcal{H}_{\alpha \beta}^{l}(r)=\left(\epsilon_{\alpha}^{*}-\epsilon_{\beta}^{*}\right) \mathcal{F}_{\alpha \beta}^{l}(r)
$$

and there is a similar relationship for $\mathcal{H}_{\gamma \delta}^{l}(r)$. The contribution of the direct interaction to $\Delta \epsilon_{M}$ vanishes such that $\Delta \epsilon_{M}=0$ for electrons of the same shell $\alpha=\beta=\gamma=\delta$, whereas the exchange interaction for $\alpha=\delta \neq \gamma=\beta$ contributes to $\Delta \epsilon_{M} \neq 0$.

The function $\mathcal{F}_{\alpha \beta}^{l}(r)$ entering Eq. (4.34) appeared earlier in the interaction energy integrals (4.15) and (4.20). As a consistency check, we observe that $\Delta \epsilon_{M}$ is equal to the lowestorder retardation correction $\Delta \epsilon_{E}$ to the Coulomb potential in the Feynman gauge. We thus conclude that the two-electron interaction energy on the order of $1 / c^{2}$ does not depend on the gauge condition. The gauge independence of the interaction energy of two electrons is thus demonstrated without assuming a specific type of mean-field potential $U(\mathbf{r})$. In positronium, the calculation of bound-state energies is performed on the order of $O\left(\alpha^{3}\right)$ using an $O\left(\alpha^{2}\right)$ approximation for the Bethe-Salpeter kernel, which is sufficient for gauge invariance on the order of $O\left(\alpha^{3}\right)$.

For noble gas atoms $\mathrm{Ne}, \mathrm{Ar}, \mathrm{Kr}, \mathrm{Xe}$, and $\mathrm{Rn}$, the difference between magnetostatic interaction energies in the Feynman and Coulomb gauges equals $0.01,0.10,1.39,5.76$, and $26.96 \mathrm{eV}$ for SEIP and 0.02, 0.25, 3.04, 12.16, and $55.72 \mathrm{eV}$ for DEIP, respectively (Niskanen et al., 2011). The variance does not exceed $60 \mathrm{eV}$. The origin of this variance can be attributed to the retardation part of the Coulomb interaction energy in the Feynman gauge. One can expect the atomic structure models to consistently determine the energy conditions for the $0 \nu 2 \mathrm{EC}$ decays with an accuracy of several tens of $\mathrm{eV}$ or better.

The proof of gauge invariance of QED of electrons and photons is based on the Ward-Green-Fradkin-Takahashi identity (Ward, 1950; Green, 1953; Fradkin, 1955; Takahashi, 1957). The diagrams without self-energy insertions into 
electron lines are known to be gauge invariant on shell;see Bjorken and Drell (1965) and Bogoliubov and Shirkov (1980). The electron self-energy part, or the mass operator $\Sigma$, depends on the gauge, which can be demonstrated explicitly in a one-loop calculation (Itzykson and Zuber, 1980) and to all orders of perturbation theory (Johnson and Zumino, 1959). The off-shell Green's functions depend on the gauge. A complete proof of the gauge invariance of the physical cross sections in QED of electrons and photons was first given by Bialynicki-Birula (1970). The equivalence of covariant Lorenz gauge and noncovariant Coulomb gauge also implies the invariance of QED with respect to Lorentz transformations. There is currently no proof of the gauge invariance of QED of multielectron atoms in higher orders of the $1 / c^{2}$ expansion. The difficulties are caused by the existence of bound states as asymptotic states of the theory. Proofs by Bjorken and Drell (1965), Bialynicki-Birula (1970), and Bogoliubov and Shirkov (1980) do not apply to diagrams whose fermion lines belong to wave functions of bound states. We believe that the uncertainties inherent in the exitation energies of multielectron atoms are entirely related to complexity in modeling the atomic systems. In the previously discussed shell model, the Feynman and Coulomb gauges provide identical results up to the order of $\alpha^{2} \mathrm{Ry}$.

\section{B. Double-electron ionization potentials in Auger spectroscopy}

To determine the energy released in the $0 \nu 2 \mathrm{EC}$ process, it is necessary to know the energy of the excited electron shell of the neutral daughter atom with two core-level vacancies and two extra valence electrons inherited from the electron shell of the parent atom. The binding energy of valence electrons usually does not exceed several $\mathrm{eV}$, which is lower than the required accuracy of $10 \mathrm{eV}$; thus, these two electrons are of no interest. To estimate the excitation energy, if this simplifies the task, they can be removed from the shell. The resulting atoms with a charge of +2 can be created in the laboratory by irradiating the substance composed of these atoms by electrons or $\mathrm{x}$ rays. Among the knocked-out electrons, one can observe electrons that arise from the so-called Auger process, schematically shown in Fig. 9. The narrow structures in the energy distribution of the knocked-out electrons correspond to transitions between the atomic levels. The study of these structures allows for the estimation of the excitation energy of the electron shell with two vacancies relevant for the $0 \nu 2 \mathrm{EC}$ decays.

When the surface of the substance is bombarded with photons or electrons with energy sufficient for ionization of one of the inner shells of the atom, a primary vacancy occurs $(\gamma)$, as shown in the left panel of Fig. 9. This vacancy is filled in a short time by an electron from a higher orbit, e.g., $L_{1}$, as illustrated in the right panel in Fig. 9. During the transition to a lower orbit, the electron interacts with the neighboring electrons via the Coulomb force and transmits to one of them energy sufficient for its knocking out to the continuum state. The resulting atom has two secondary vacancies $\alpha$ and $\beta$ plus one ejected Auger electron. Let $\epsilon_{\gamma}^{*}$ be the binding energy of the first knocked-out electron (photoelectron). The energy of the shell with one vacancy equals $\epsilon_{\gamma}^{*}$. If $\epsilon_{\alpha}^{*}$ and $\epsilon_{\beta}^{*}$ are the energies
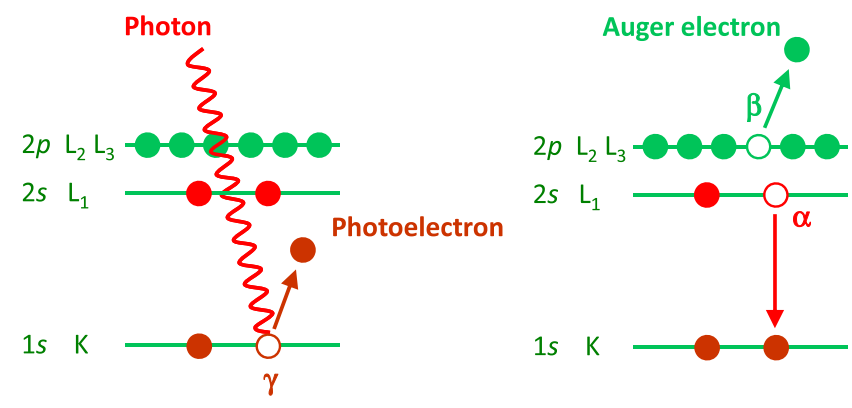

FIG. 9. Schematic representation of Auger-electron knockout by x-ray photons $\left(q^{2}=0\right)$ or by virtual photons $\left(q^{2} \neq 0\right)$ emitted by electrons irradiating the surface of a sample. In the first stage, the $K$ shell electron is excited to a continuum state (left panel). The resulting vacancy $\gamma$ is filled with an electron from the $L_{1}$ shell $(\alpha)$, which is accompanied by the radiation of a virtual photon to knock out an electron $\beta$ from the $L_{2}$ or $L_{3}$ shell (right panel). The excitation energy $\varepsilon_{\gamma}^{*}$ goes to the formation of the vacancies $\alpha$ and $\beta$ and the kinetic energy $\varepsilon_{\text {kin }}^{\mathrm{A}}$ of the Auger electron. By measuring the energy of the knocked-out electrons, it is possible to determine the peaks at energy values of the corresponding transitions.

of single vacancies, the energy of the shell with two vacancies is the sum of single excitation energies $\epsilon_{\alpha}^{*}+\epsilon_{\beta}^{*}$ plus the Coulomb interaction of holes and relativistic and relaxation effects, which we denote by $\Delta \epsilon_{\alpha \beta}^{*}$. The kinetic energy of the photoelectron equals

$$
\epsilon_{\mathrm{kin}}=\omega-\epsilon_{\gamma}^{*}-\phi
$$

where $\omega$ is the photon energy and $\phi$ is the work function. In solid-phase systems $\phi$ is equal to a few eV, and in vapor-phase systems $\phi=0$. The energy of the Auger electron is also determined by the conservation of energy

$$
\epsilon_{\mathrm{kin}}^{\mathrm{A}}=\epsilon_{\gamma}^{*}-\epsilon_{\alpha \beta}^{*}-\phi
$$

where

$$
\epsilon_{\alpha \beta}^{*}=\epsilon_{\alpha}^{*}+\epsilon_{\beta}^{*}+\Delta \epsilon_{\alpha \beta}^{*} .
$$

These equations show that the photoelectron energy spectrum contains information about the single-hole excitation energies, whereas the Auger-electron energy spectrum allows for the measurement of the two-hole excitation energies of electron shells. These energies occur in the energy balance of the $0 \nu 2 \mathrm{EC}$ transitions. We consider the experimental values of excitation energies $\epsilon_{\alpha \beta}^{*}$ to estimate the probability of $0 \nu 2 \mathrm{EC}$ decays as the preferable choice. As can be seen also from Fig. 9, not all combinations of vacancies are available for the measurement. Auger electrons are associated with vacancies $n_{\alpha} \geq 2$ and $n_{\beta} \geq 2$. When experimental data are not available, we perform calculations using the GRASP2K package.

The main question is whether or not it is possible to calculate the two-hole excitation energy of atoms with an accuracy of $10 \mathrm{eV}$, which is attainable experimentally in 
TABLE I. Total binding energies of neutral noble gas atoms in eV. Column 2 shows our calculations using the GRASP2K package. The last three columns show the results of Lu et al. (1971), Desclaux (1973), and Huang et al. (1976).

\begin{tabular}{lrrrr}
\hline \hline Element & GRASP2K & Lu et al. (1971) & Desclaux (1973) & Huang et al. (1976) \\
\hline $\mathrm{Ne}$ & 3501.1 & 3472.0 & 3501.4 & 3500.8 \\
$\mathrm{Ar}$ & 14380.1 & 14072.6 & 14382.3 & 14382.1 \\
$\mathrm{Kr}$ & 75823.8 & 75739.0 & 75845.8 & 75851.7 \\
$\mathrm{Xe}$ & 202379.5 & 202402.7 & 202465.3 & 202498.4 \\
$\mathrm{Rn}$ & 640906.9 & 641899.1 & 641348.1 & 641591.6 \\
\hline \hline
\end{tabular}

Penning-trap mass spectrometry and is typical of the natural widths of two-hole excitations.

To estimate the magnitude of uncertainty, we first consider the total binding energy of inert gas atoms. Table I summarizes the results of our calculations performed using the GRASP2K software package. These results are compared to those from Lu et al. (1971), Desclaux (1973), and Huang et al. (1976). For light atoms, the variance is negligible. For the mediumheavy nucleus $\mathrm{Xe}$, the variance does not exceed $120 \mathrm{eV}$. For $\mathrm{Rn}$, the variance does not exceed $1 \mathrm{keV}$; the case of heavy atoms should be treated with caution.

An estimate of the uncertainties in the energy of single-hole excitations can be obtained from Table II, where the results of the GRASP2K package are compared with the data of Larkins (1977), obtained within the framework of a general semiempirical method that takes into account experimental data on the binding energy of electron subshells and results of HartreeFock atomic calculations. For heavy atoms, the mismatch is basically of the order of $10 \mathrm{eV}$ or less, and it is always less than $20 \mathrm{eV}$. The claimed accuracy of Larkins's data is a few eV for light atoms and $10 \mathrm{eV}$ for heavy atoms.

The energies of two-hole excitations of the inert gas atoms of $\mathrm{Ne}$ and $\mathrm{Ar}$ are collected in Table III; these data can be further supplemented by the results of calculating the energy of two-hole excitations of $\mathrm{Kr}, \mathrm{Xe}$, and $\mathrm{Rn}$. The results for $2 \leq n_{\alpha} \leq 3$ and $2 \leq n_{\beta} \leq 3$ are compared to the semiempirical values extracted from the energy spectrum of Auger electrons (Larkins, 1977). The second column of these tables lists the total angular momentum $J$ of the two holes; the sums of the energies of the single-particle excitations and the energy of two-hole excitation, determined on the basis of Auger spectroscopy data, are then presented. Column 5 reports two-hole excitation energy according to our calculations using the GRASP2K package. The last column contains quantum numbers of the pair $(n, 2 j, l)$. We remark that $n$ and $l$ are integers, $j$ are half integers, and $2 j$ are odd. Mixing occurs if some states of the pair arise in two or more combinations. The mixing matrix is presented in column 6. For mixed states, column 5 lists the energy eigenvalues. For example, for the Ar atom, the vacancies $|(210)(211)\rangle \equiv\left|2 s_{1 / 2} 2 p_{1 / 2}\right\rangle$ and $|(210)(231)\rangle \equiv\left|2 s_{1 / 2} 2 p_{3 / 2}\right\rangle$ with $J=1$ are mixed, whereby the eigenstates have the form

$|J=1 ; 631.2 \mathrm{eV}\rangle=0.612|(210)(211)\rangle+0.791|(210)(231)\rangle$,
$|J=1 ; 606.8 \mathrm{eV}\rangle=0.791|(210)(211)\rangle-0.612|(210)(231)\rangle$.

The energy of these states are 631.2 and $606.8 \mathrm{eV}$, respectively. Larkins (1977) did not take into account such mixing. In the previous example, the difference between the energies of the holes with and without the mixing is small. In the case of Xe, the deviation from the semiempirical values of Larkins (1977) does not exceed $10 \mathrm{eV}$, whereas for $\mathrm{Rn}$ the deviation does not exceed $40 \mathrm{eV}$. The deviation is negligible for light atoms.

The energies of the electron shells of noble gas atoms with double- $K$ holes were calculated by Niskanen et al. (2011). We obtain good agreement for $\mathrm{Ne}, \mathrm{Ar}, \mathrm{Kr}$, and $\mathrm{Xe}$, but the results are noticeably different for Rn. Niskanen et al. (2011) gave

TABLE II. Single-electron ionization potentials for the noble gas series from Ne to Rn in eV. The second and sixth columns list the hole quantum numbers: $n$ is the principal quantum number, $j$ is the total angular momentum, and $l$ is the orbital momentum. Columns 3 and 7 present results of our calculations using the GRASP2K package. Columns 4 and 8 list the results of Larkins (1977).

\begin{tabular}{|c|c|c|c|c|c|c|c|}
\hline Element & $n 2 j l$ & GRASP2K & Larkins (1977) & Element & $n 2 j l$ & GRASP2K & Larkins (1977) \\
\hline $\mathrm{Ne}$ & 110 & 869.3 & 870.1 & & 310 & 1151.4 & 1148.7 \\
\hline \multirow[t]{4}{*}{$\mathrm{Ar}$} & 110 & 3205.8 & 3206.0 & & 311 & 1005.5 & 1002.1 \\
\hline & 210 & 327.0 & 326.3 & & 331 & 943.3 & 940.6 \\
\hline & 211 & 250.3 & 250.7 & & 410 & 222.6 & 213.3 \\
\hline & 231 & 248.2 & 248.6 & & 411 & 169.2 & 145.5 \\
\hline \multirow[t]{7}{*}{$\mathrm{Kr}$} & 110 & 14325.9 & 14325.6 & & 431 & 156.8 & 145.5 \\
\hline & 210 & 1930.7 & 1921.0 & $\mathrm{Rn}$ & 110 & 98390.5 & 98397.0 \\
\hline & 211 & 1732.3 & 1727.2 & & 210 & 18061.8 & 18048.0 \\
\hline & 231 & 1679.3 & 1674.9 & & 211 & 17335.6 & 17328.0 \\
\hline & 310 & 295.9 & 292.1 & & 231 & 14614.2 & 14610.0 \\
\hline & 311 & 225.1 & 221.8 & & 310 & 4489.7 & 4473.0 \\
\hline & 331 & 217.0 & 214.5 & & 311 & 4164.4 & 4150.0 \\
\hline \multirow[t]{4}{*}{$\mathrm{Xe}$} & 110 & 34562.4 & 34564.4 & & 331 & 3542.8 & 3529.0 \\
\hline & 210 & 5458.8 & 5452.8 & & 410 & 1104.9 & 1090.0 \\
\hline & 211 & 5107.5 & 5103.7 & & 411 & 961.3 & 944.0 \\
\hline & 231 & 4786.9 & 4782.2 & & 431 & 803.9 & 790.0 \\
\hline
\end{tabular}


TABLE III. Double-electron ionization potentials for the noble gas atoms Ne and Ar in eV. Column 2 lists the total angular momentum of the pair. Column 3 presents the sum of excitation energies of the single-hole states. The values of $\epsilon_{\alpha}^{*}$ and $\epsilon_{\beta}^{*}$ are from Larkins (1977). Column 4 reports the double-electron ionization potentials extracted from the Auger-electron spectroscopy data from Larkins (1977). The Auger transitions allow for the determination of the excitation energies of two-hole states with $n_{\alpha}, n_{\beta} \geq 2$. Column 5 presents the results of our calculations using the GRASP2K package. The principal quantum number $n$, the total angular momentum $j$, and the orbital momentum $l$ of electron holes $\alpha$ and $\beta$ are reported in the last columns. Column 6 presents the mixing matrix of the two-hole states, which gives the energy eigenstates with definite $J$. The energy levels in columns 3 and 4 neglect mixing and are ordered in the coincidence with column 5, i.e., for a unit-mixing matrix.

\begin{tabular}{|c|c|c|c|c|c|c|}
\hline Element & $J$ & $\epsilon_{\alpha}^{*}+\epsilon_{\beta}^{*}$ & $\epsilon_{\alpha \beta}^{\mathrm{A}}$ & $\epsilon_{\alpha \beta}^{*}$ & $U(\cdot \mid \alpha \beta)$ & $(n 2 j l)_{\alpha}(n 2 j l)_{\beta}$ \\
\hline $\mathrm{Ne}$ & 0 & 1740.2 & & 1862.1 & 1.000 & 110110 \\
\hline \multirow[t]{17}{*}{$\mathrm{Ar}$} & 0 & 6412.0 & & 6653.5 & 1.000 & 110110 \\
\hline & 0 & 3532.3 & & 3588.7 & 1.000 & 110210 \\
\hline & 1 & 3532.3 & & 3579.8 & 1.000 & 110210 \\
\hline & 0 & 3456.7 & & 3513.3 & 1.000 & 110211 \\
\hline & 1 & 3456.7 & & 3522.0 & 0.6690 .743 & 110211 \\
\hline & & 3454.6 & & 3511.9 & $0.743-0.669$ & 110231 \\
\hline & 2 & 3454.6 & & 3510.1 & 1.000 & 110231 \\
\hline & 0 & 652.6 & 695.4 & 695.0 & 1.000 & 210210 \\
\hline & 0 & 577.0 & 606.7 & 607.6 & 1.000 & 210211 \\
\hline & 1 & 577.0 & 630.0 & 631.2 & 0.6120 .791 & 210211 \\
\hline & & 574.9 & 605.9 & 606.8 & $0.791-0.612$ & 210231 \\
\hline & 2 & 574.9 & 604.6 & 605.3 & 1.000 & 210231 \\
\hline & 0 & 501.4 & 553.8 & 557.0 & 0.6690 .743 & 211211 \\
\hline & & 497.2 & 539.3 & 538.5 & $0.743-0.669$ & 231231 \\
\hline & 1 & 499.3 & 538.9 & 538.0 & 1.000 & 211231 \\
\hline & 2 & 499.3 & 545.8 & 545.1 & $0.881-0.474$ & 211231 \\
\hline & & 497.2 & 537.4 & 536.3 & 0.4740 .881 & 231231 \\
\hline
\end{tabular}

$\epsilon_{K K}^{*}=198912.6 \mathrm{eV}$, whereas our calculations yield the value of $198568.3 \mathrm{eV}$, which is $344 \mathrm{eV}$ less. Niskanen et al. (2011) did not take into account QED corrections due to the electron self-energy and the vacuum polarization. If these effects are neglected in our calculations, then a value of $198977.8 \mathrm{eV}$ is obtained; this value differs by only $65 \mathrm{eV}$ from the result of Niskanen et al. (2011). The uncertainty in two-hole excitation energies of heavy atoms is thus found to be about $60 \mathrm{eV}$, which is higher than the $0 \nu 2 \mathrm{EC}$ target of $10 \mathrm{eV}$.

The $0 \nu 2$ EC half-lives are estimated in Secs. V and VIII by neglecting the mixing. The two-hole excitation energies of atoms with arbitrary $Z$ are determined using the data for $\mathrm{Ne}$ and $\mathrm{Ar}$ (Table III) and also $\mathrm{Kr}, \mathrm{Xe}$, and $\mathrm{Rn}$ by means of interpolation $\epsilon_{\alpha \beta}^{*}=a Z^{b}$ between the neighboring noble gas atoms with the same vacancies.

\section{Summary}

The probability of capture of orbital electrons by the nucleus depends on the value of the electron wave functions inside the nucleus. The values of upper and lower components of the Dirac electron wave functions of neutral atoms inside the nuclei were tabulated by Band and Trzhaskovskaya (1986), who accounted for the electron screening using the relativistic Dirac-Fock-Slater and Dirac-Fock potentials. The $n s_{1 / 2}$ waves are dominant. The $n p_{1 / 2}$ waves can be found to be enhanced compared to the $n p_{3 / 2}$ waves. The nonrelativistic solutions of the $n p_{3 / 2}$ waves are close to the relativistic ones; see also Grant (2007), Fig. 1.2. The electron capture from the $n p_{3 / 2}$ orbits is therefore suppressed. The results of Band and Trzhaskovskaya (1986) are in excellent agreement with the results provided by the GRASP $2 \mathrm{~K}$ software package.
The resonant enhancement of the $0 \nu 2 \mathrm{EC}$ decays occurs when the excitation energies of the parent and intermediate daughter atoms are degenerate with an accuracy of about $10 \mathrm{eV}$. This scale characterizes the typical excitation width of the atomic shells. Accuracy of about $10 \mathrm{eV}$ is achievable on Penning traps when measuring mass difference of ionized atoms. To identify the resonant $0 \nu 2 \mathrm{EC}$ with the same high accuracy, information about double-hole excitations of electron shells is required.

The two-hole interactions provide a dominant contribution to the energy of excited electron shells, but not the only contribution. Open vacancies in the occupation numbers affect the energy of all atomic levels. The GRASP2K package calculates the structure of electron shells based on the Green's function method of QED, which offers a simple and clear description of various approximations.

Accuracy of up to $10 \mathrm{eV}$ is readily achievable when determining theoretically single-hole excitation energies. We demonstrated independence on the gauge of single-hole excitation energies of the order of $\alpha^{2} \mathrm{Ry}$. One of the challenges of the atomic theory is the proof of gauge invariance of QED of multielectron atoms in all orders of perturbation theory. Double-hole excitation energies can be determined theoretically with an accuracy of $60 \mathrm{eV}$ or better. The upper bound of the possible error is still higher than that required to identify the resonant $0 \nu 2 \mathrm{EC}$ unambiguously. In calculations with atomic shell structure, the uncertainties are associated with complexity of the bound-state problem for multielectron atoms. In many cases, the resonant parameter of $0 \nu 2 \mathrm{EC}$ can be extracted from the experimental data on Auger spectroscopy. When normalization to the experimental values is not possible, quantum chemistry codes such as GRASP $2 \mathrm{~K}$ can be used to get the missing information. 


\section{NUCLEAR MATRIX ELEMENTS}

In this section we describe how nuclear structure affects the half-life of the $0 \nu 2 \mathrm{EC}$ process and review the available calculations of the related NMEs. We also add new NME calculations to complement the list of the evaluated $0 \nu 2 \mathrm{EC}$ cases.

\section{A. Overview of the calculated nuclear matrix elements in $0 \nu 2 \mathrm{EC}$}

Nuclear structure is heavily involved in the decay amplitude (3.21) through the appropriate NMEs (Suhonen, 2012a). These NMEs have been computed in various theory frameworks as described next. A representative list of the calculations is displayed in Table IV. In the table the available estimated lowest and highest limits for the near-resonant $0 \nu 2 \mathrm{EC}$ half-lives (last two columns) are listed. In the evaluation of the half-lives Eq. (8.1) was adopted and the NMEs $M^{0 \nu}$ from column 4 of Table IV were used, in addition to the degeneracy parameters taken from Tables VI and VII. The theory frameworks used to derive these NMEs are also given (column 5), along with the corresponding reference (column 6). The $Q_{2 \mathrm{EC}}$-value measurements for the evaluation of the degeneracy parameters were performed using modern Penning-trap techniques; see Sec. VI.

In practically all the listed cases the decay rates are suppressed by the rather sizable magnitude of the ratio $\left(\Delta / \Gamma_{\alpha \beta}\right)^{2}$, where $\Gamma_{\alpha \beta} \sim 10 \mathrm{eV}$ is the typical deexcitation width of the excited electron shells with the electron vacancies $\alpha$ and $\beta$. Decays to $0^{+}$states are favored over the decays to $2^{+}$ or $1^{-}, 2^{-}, 3^{-}$, etc., states due to the involved nuclear wave functions and/or higher-order transitions. A further suppression stems from nuclear deformation. This suppression is

TABLE IV. The "sQRPA" and "dQRPA" entries in column 5 denote the spherical pnQRPA and deformed QRPA outlined at the beginning of Sec. V.B.1 and in Sec. V.B.2, respectively. Furthermore, the symbol "IBM-2" denotes the microscopic interacting boson model of Sec. V.B.3 and the symbol "EDF" denotes the energy-density functional method of Sec. V.B.4. In the case of the multiple-commutator model (MCM of Sec. V.B.1) we have chosen to quote the results obtained by the use of the UCOM short-range correlations (UCOM s.r.c.), which are the most realistic ones (Kortelainen et al., 2007). The UCOM s.r.c. have also been used in the sQRPA, dQRPA, and EDF calculations. The "qp estimate" in the fifth column denotes the procedure outlined in Sec. V.C. The last two columns give the minimum and maximum half-lives (in years) calculated using Eq. (8.1) with $K_{Z}$ of Eq. (3.19), $m_{\beta \beta}=100 \mathrm{meV}$, and $g_{A}=1.27$. The excitation energies are given in keV.

\begin{tabular}{|c|c|c|c|c|c|c|c|}
\hline Transition & $J_{f}^{\pi}$ & $M_{A, Z-2}^{*}-M_{A, Z-2}$ & $M^{0 \nu}$ & Model & Reference & $T_{1 / 2}^{\min }$ & $T_{1 / 2}^{\max }$ \\
\hline${ }_{34}^{74} \mathrm{Se} \rightarrow{ }_{32}^{74} \mathrm{Ge}^{* *}$ & $2_{2}^{+}$ & $1204.205 \pm 0.007$ & $3.22 \times 10^{-4}$ & $\mathrm{MCM}$ & Kolhinen et al. (2010) & $1.2 \times 10^{46}$ & $1.2 \times 10^{46}$ \\
\hline${ }_{44}^{96} \mathrm{Ru} \rightarrow{ }_{42}^{96} \mathrm{Mo}^{* *}$ & $\left(0^{+}\right)$ & $2712.68 \pm 0.10$ & 5.57 & $\mathrm{MCM}$ & Suhonen (2012b) & $2.2 \times 10^{32}$ & $6.8 \times 10^{32}$ \\
\hline${ }_{48}^{106} \mathrm{Cd} \rightarrow{ }_{44}^{106} \mathrm{Pd}^{* *}$ & $\left(0^{+}\right)$ & $2717.59 \pm 0.21$ & $3.38-3.48$ & $\mathrm{MCM}$ & Suhonen (2011) & $5.3 \times 10^{31}$ & $7.0 \times 10^{31}$ \\
\hline${ }_{50}^{112} \mathrm{Sn} \rightarrow{ }_{48}^{112} \mathrm{Cd}^{* *}$ & $\left(0^{+}\right)$ & $2988 \pm 8$ & 4.76 & Estimate & Rahaman et al. (2009) & $3.5 \times 10^{35}$ & $3.8 \times 10^{35}$ \\
\hline \multirow[t]{2}{*}{${ }_{54}^{124} \mathrm{Xe} \rightarrow{ }_{52}^{124} \mathrm{Te}^{* *}$} & $0_{4}^{+}$ & $2153.29 \pm 0.03$ & $1.11-1.30$ & MCM & Suhonen (2013) & $7.3 \times 10^{35}$ & $7.3 \times 10^{35}$ \\
\hline & & & 0.297 & IBM-2 & $\begin{array}{c}\text { Kotila, Barea, and } \\
\text { Iachello (2014) }\end{array}$ & $1.2 \times 10^{37}$ & $1.2 \times 10^{37}$ \\
\hline${ }_{58}^{136} \mathrm{Ce} \rightarrow{ }_{56}^{136} \mathrm{Ba}^{* *}$ & $0_{4}^{+}$ & $2315.32 \pm 0.07$ & 0.68 & $\mathrm{MCM}$ & Kolhinen et al. (2011) & $3.3 \times 10^{32}$ & $4.3 \times 10^{32}$ \\
\hline \multirow[t]{4}{*}{${ }_{64}^{152} \mathrm{Gd} \rightarrow{ }_{62}^{152} \mathrm{Sm}^{*}$} & $0_{1}^{+}$ & 0 & $7.21-7.59$ & sQRPA & $\begin{array}{l}\text { Šimkovic, Krivoruchenko, } \\
\text { and Faessler (2011) }\end{array}$ & $6.8 \times 10^{27}$ & $6.8 \times 10^{28}$ \\
\hline & & & $2.67-3.23$ & dQRPA & Fang et al. (2012) & $4.3 \times 10^{28}$ & $4.3 \times 10^{29}$ \\
\hline & & & 2.445 & IBM-2 & $\begin{array}{c}\text { Kotila, Barea, and } \\
\text { Iachello (2014) }\end{array}$ & $6.0 \times 10^{28}$ & $6.0 \times 10^{29}$ \\
\hline & & & $0.89-1.07$ & EDF & $\begin{array}{l}\text { Rodríguez and Martínez- } \\
\text { Pinedo (2010) }\end{array}$ & $3.8 \times 10^{29}$ & $3.8 \times 10^{30}$ \\
\hline \multirow[t]{6}{*}{${ }_{66}^{156} \mathrm{Dy} \rightarrow{ }_{64}^{156} \mathrm{Gd}^{* *}$} & $0_{1}^{+}$ & 0 & 3.175 & IBM-2 & $\begin{array}{c}\text { Kotila, Barea, and } \\
\text { Iachello (2014) }\end{array}$ & $7.3 \times 10^{34}$ & $7.3 \times 10^{34}$ \\
\hline & $0_{2}^{+}$ & $1049.487 \pm 0.002$ & 1.749 & IBM-2 & $\begin{array}{c}\text { Kotila, Barea, and } \\
\text { Iachello (2014) }\end{array}$ & $4.8 \times 10^{34}$ & $4.8 \times 10^{34}$ \\
\hline & $0_{3}^{+}$ & $1168.186 \pm 0.007$ & 0.466 & IBM-2 & $\begin{array}{c}\text { Kotila, Barea, and } \\
\text { Iachello (2014) }\end{array}$ & $5.0 \times 10^{35}$ & $5.0 \times 10^{35}$ \\
\hline & $0_{4}^{+}$ & $1715.192 \pm 0.005$ & 0.311 & IBM-2 & $\begin{array}{c}\text { Kotila, Barea, and } \\
\text { Iachello (2014) }\end{array}$ & $7.5 \times 10^{34}$ & $7.5 \times 10^{34}$ \\
\hline & $0_{5}^{+}$ & $1851.239 \pm 0.007$ & 0.346 & IBM-2 & $\begin{array}{c}\text { Kotila, Barea, and } \\
\text { Iachello (2014) }\end{array}$ & $4.8 \times 10^{33}$ & $4.8 \times 10^{33}$ \\
\hline & $0_{6}^{+}$ & $1988.5 \pm 0.2$ & 0.3 & Estimate & This work & $1.1 \times 10^{28}$ & $9.5 \times 10^{32}$ \\
\hline \multirow[t]{2}{*}{${ }_{68}^{164} \mathrm{Er} \rightarrow{ }_{66}^{164} \mathrm{Dy}^{* *}$} & $0_{1}^{+}$ & 0 & $5.94-6.12$ & sQRPA & $\begin{array}{l}\text { Šimkovic, Krivoruchenko, } \\
\text { and Faessler (2011) }\end{array}$ & $3.3 \times 10^{31}$ & $3.5 \times 10^{31}$ \\
\hline & & & $2.27-2.64$ & dQRPA & Fang et al. (2012) & $2.0 \times 10^{32}$ & $2.1 \times 10^{32}$ \\
\hline
\end{tabular}




\begin{tabular}{|c|c|c|c|c|c|c|c|}
\hline Transition & $J_{f}^{\pi}$ & $M_{A, Z-2}^{*}-M_{A, Z-2}$ & $M^{0 \nu}$ & Model & Reference & $T_{1 / 2}^{\min }$ & $T_{1 / 2}^{\max }$ \\
\hline \multirow{6}{*}{${ }_{74}^{180} \mathrm{~W} \rightarrow{ }_{72}^{180} \mathrm{Hf}^{* *}$} & \multirow{6}{*}{$0_{1}^{+}$} & \multirow{6}{*}{0} & 3.952 & IBM-2 & $\begin{array}{c}\text { Kotila, Barea, and } \\
\text { Iachello (2014) }\end{array}$ & $7.8 \times 10^{31}$ & $8.0 \times 10^{31}$ \\
\hline & & & $0.50-0.64$ & EDF & $\begin{array}{l}\text { Rodríguez and Martínez- } \\
\text { Pinedo (2010) }\end{array}$ & $3.8 \times 10^{33}$ & $4.0 \times 10^{33}$ \\
\hline & & & $5.56-5.79$ & sQRPA & $\begin{array}{l}\text { Šimkovic, Krivoruchenko, } \\
\text { and Faessler (2011) }\end{array}$ & $1.4 \times 10^{29}$ & $1.8 \times 10^{29}$ \\
\hline & & & $1.79-2.05$ & dQRPA & Fang et al. (2012) & $1.2 \times 10^{30}$ & $1.6 \times 10^{30}$ \\
\hline & & & 4.672 & IBM-2 & $\begin{array}{l}\text { Kotila, Barea, and } \\
\text { Iachello (2014) }\end{array}$ & $2.1 \times 10^{29}$ & $2.8 \times 10^{29}$ \\
\hline & & & $0.38-0.58$ & EDF & $\begin{array}{l}\text { Rodríguez and Martínez- } \\
\text { Pinedo (2010) }\end{array}$ & $2.0 \times 10^{31}$ & $2.5 \times 10^{31}$ \\
\hline \multirow[t]{4}{*}{${ }_{76}^{184} \mathrm{Os} \rightarrow{ }_{74}^{184} \mathrm{~W}^{* *}$} & $0_{2}^{+}$ & $1002.48 \pm 0.04$ & 0.631 & $\mathrm{EDF}$ & Smorra et al. (2012) & $6.8 \times 10^{33}$ & $6.8 \times 10^{33}$ \\
\hline & $(0)_{3}^{+}$ & $1322.152 \pm 0.022$ & 0.504 & $\mathrm{EDF}$ & Smorra et al. (2012) & $6.0 \times 10^{30}$ & $1.2 \times 10^{31}$ \\
\hline & $2_{5}^{+}$ & $1431.02 \pm 0.05$ & 0.14 & $\mathrm{MCM}$ & This work & $4.5 \times 10^{32}$ & $4.5 \times 10^{37}$ \\
\hline & $(0)_{5}^{+}$ & $1713.47 \pm 0.10$ & 0.163 & EDF & Smorra et al. (2012) & $1.7 \times 10^{35}$ & $1.7 \times 10^{35}$ \\
\hline \multirow{2}{*}{${ }_{76}^{190} \mathrm{Pt} \rightarrow{ }_{74}^{190} \mathrm{Os}^{* *}$} & $1^{+}$ & $1326.9 \pm 0.5$ & 1.1 & $\mathrm{MCM}$ & This work & $3.3 \times 10^{26}$ & $1.6 \times 10^{30}$ \\
\hline & $\left(0_{3}\right)^{+}$ & $1382.4 \pm 0.2$ & 4.7 & $\mathrm{MCM}$ & This work & $1.0 \times 10^{30}$ & $6.5 \times 10^{30}$ \\
\hline \multirow{2}{*}{${ }_{64}^{148} \mathrm{Gd} \rightarrow{ }_{62}^{148} \mathrm{Sm}^{* *}$} & $\left(0^{+}\right)$ & $3004 \pm 3$ & 0.071 & qp estimate & This work & $5.3 \times 10^{28}$ & $1.5 \times 10^{35}$ \\
\hline & $\left(1^{+}\right)$ & & 0.031 & qp estimate & This work & $8.3 \times 10^{28}$ & $2.5 \times 10^{35}$ \\
\hline \multirow{4}{*}{$\begin{array}{l}{ }_{64}^{150} \mathrm{Gd} \rightarrow{ }_{62}^{150} \mathrm{Sm}^{* *} \\
{ }_{66}^{154} \mathrm{Dy} \rightarrow{ }_{64}^{154} \mathrm{Gd}^{* *}\end{array}$} & $0^{+}$ & $1255.400 \pm 0.022$ & 0.066 & qp estimate & This work & $4.0 \times 10^{29}$ & $1.6 \times 10^{36}$ \\
\hline & $\left(0^{+}\right)$ & 3153.1 & 0.068 & qp estimate & This work & $6.8 \times 10^{34}$ & $2.8 \times 10^{35}$ \\
\hline & $\left(0^{+}\right)$ & $3154.8 \pm 0.4$ & 0.068 & qp estimate & This work & $6.3 \times 10^{34}$ & $2.8 \times 10^{35}$ \\
\hline & $\left(1^{+}\right)$ & $3264.42 \pm 0.21$ & 0.030 & qp estimate & This work & $7.5 \times 10^{28}$ & $3.8 \times 10^{35}$ \\
\hline \multirow[t]{5}{*}{${ }_{80}^{194} \mathrm{Hg} \rightarrow{ }_{78}^{194} \mathrm{Pt}^{* *}$} & $\left(0^{+}\right)$ & $2450 \pm 5$ & 0.017 & qp estimate & This work & $2.5 \times 10^{29}$ & $1.2 \times 10^{36}$ \\
\hline & $\left(1^{+}\right)$ & & 0.004 & qp estimate & This work & $1.4 \times 10^{30}$ & $6.3 \times 10^{36}$ \\
\hline & $\left(0^{+}\right)$ & $2472 \pm 5$ & 0.017 & qp estimate & This work & $2.5 \times 10^{29}$ & $1.2 \times 10^{36}$ \\
\hline & $\left(1^{+}\right)$ & & 0.004 & qp estimate & This work & $1.4 \times 10^{30}$ & $2.8 \times 10^{36}$ \\
\hline & $\left(1^{+}\right)$ & $2500 \pm 10$ & 0.004 & qp estimate & This work & $1.4 \times 10^{30}$ & $6.5 \times 10^{36}$ \\
\hline \multirow{2}{*}{${ }_{82}^{202} \mathrm{~Pb} \rightarrow{ }_{78}^{202} \mathrm{Hg}^{* *}$} & $0_{2}^{+}$ & $1411.37 \pm 0.12$ & 0.011 & qp estimate & This work & $1.7 \times 10^{30}$ & $1.6 \times 10^{37}$ \\
\hline & $\left(1^{+}\right)$ & $1347.92 \pm 0.07$ & 0.003 & qp estimate & This work & $4.0 \times 10^{33}$ & $4.8 \times 10^{36}$ \\
\hline
\end{tabular}

typically a few tens of percent (Ejiri, Suhonen, and Zuber, 2019) but can be even stronger, factor of 2 to 3, for large deformations (Delion and Suhonen, 2017). The radial wave functions of electrons in low-lying atomic states on the surface of medium-heavy nuclei are from Band and Trzhaskovskaya (1986). In relativistic theory, the electron capture from the $n s_{1 / 2}$ states is dominant, the amplitude of electron capture from the $n p_{1 / 2}$ states is suppressed by about an order of magnitude, and the amplitude of electron capture from the $j \geq 3 / 2$ state appears to be suppressed by several orders of magnitude (Kolhinen et al., 2010; Krivoruchenko et al., 2011).

There are some favorable values of degeneracy parameters listed in Table VII, as for the transitions ${ }^{106} \mathrm{Cd} \rightarrow$ ${ }^{106} \mathrm{Pd}\left[(2,3)^{-}\right]$and ${ }^{156} \mathrm{Dy} \rightarrow{ }^{156} \mathrm{Gd}\left(1^{-}, 2^{+}\right)$, but the associated nuclear matrix elements have not yet been evaluated. At the moment the most favorable case with a half-life estimate of $\gtrsim 5 \times 10^{28} \mathrm{yr}$ is the case ${ }^{152} \mathrm{Gd} \rightarrow{ }^{152} \mathrm{Sm}\left(0_{\mathrm{gs}}^{+}\right)$, which corresponds to a decay transition to the ground state.

All the presently identified favorable $0 \nu 2 \mathrm{EC}$ cases are in the regions of relatively strong nuclear deformation so that a proper handling of this degree of freedom poses a challenge to the nuclear-theory frameworks.

\section{B. Overview of the calculation frameworks}

In the analyses of the near-resonant $0 \nu 2 \mathrm{EC}$ decay transitions the adopted many-body frameworks include the quasiparticle random-phase approximation (QRPA) and its higher-QRPA versions: multiple-commutator model (MCM), the deformed QRPA, the microscopic interacting boson model (IBM-2), and the energy-density functional (EDF) method. The MCM and deformed QRPA frameworks compute the $0 \nu 2 \mathrm{EC}$-decay NME explicitly, including the contributions from the virtual states of the intermediate nucleus. The other two models, IBM-2 and EDF, resort to the closure approximation where the sum over the intermediate states, with the appropriate energy denominator, has been suppressed by assuming an average excitation energy in the denominator and then using the closure over the complete set of intermediate virtual states. All these models are briefly described later. 
The NME for the near-resonant $0 \nu 2 \mathrm{EC}$ decay to the $0^{+}$final states is written as a linear combination of the Gamow-Teller (GT), Fermi (F), and tensor (T) NMEs as

$$
M^{2 \mathrm{EC}}=M_{\mathrm{GT}}^{2 \mathrm{EC}}-\left(\frac{g_{\mathrm{V}}}{g_{\mathrm{A}}}\right)^{2} M_{\mathrm{F}}^{2 \mathrm{EC}}+M_{\mathrm{T}}^{2 \mathrm{EC}} .
$$

In the MCM and deformed QRPA frameworks the transitions through the virtual states of the intermediate nucleus are treated explicitly. Then the double Fermi, Gamow-Teller, and tensor nuclear matrix elements can be written as

$$
\begin{array}{r}
M_{\mathrm{F}}^{2 \mathrm{EC}}=\sum_{k}\left(J_{f}^{+}\left\|\sum_{m n} h_{\mathrm{F}}\left(r_{m n}, E_{k}\right)\right\| 0_{i}^{+}\right), \\
M_{\mathrm{GT}}^{2 \mathrm{EC}}=\sum_{k}\left(J_{f}^{+}\left\|\sum_{m n} h_{\mathrm{GT}}\left(r_{m n}, E_{k}\right)\left(\boldsymbol{\sigma}_{m} \cdot \boldsymbol{\sigma}_{n}\right)\right\| 0_{i}^{+}\right), \\
M_{\mathrm{T}}^{2 \mathrm{EC}}=\sum_{k}\left(J_{f}^{+}\left\|\sum_{m n} h_{\mathrm{T}}\left(r_{m n}, E_{k}\right) S_{m n}^{\mathrm{T}}\right\| 0_{i}^{+}\right),
\end{array}
$$

where the tensor operator reads

$$
S_{m n}^{\mathrm{T}}=3\left[\left(\boldsymbol{\sigma}_{m} \cdot \hat{\mathbf{r}}_{m n}\right)\left(\boldsymbol{\sigma}_{n} \cdot \hat{\mathbf{r}}_{m n}\right)\right]-\boldsymbol{\sigma}_{m} \cdot \boldsymbol{\sigma}_{n} .
$$

The summations over $k$ in Eqs. (5.2)-(5.4) run over all the states of the intermediate odd-odd nucleus, $r_{m n}=\left|\mathbf{r}_{m}-\mathbf{r}_{n}\right|$ is the relative distance between the two decaying protons, labeled $m$ and $n$, and $\hat{\mathbf{r}}_{m n}=\left(\mathbf{r}_{m}-\mathbf{r}_{n}\right) / r_{m n}$. The neutrino potentials $h_{K}\left(r_{m n}, E_{k}\right), K=\mathrm{F}, \mathrm{GT}$, T were given by Suhonen (2012a). The ground state of the initial even-even nucleus is denoted by $0_{i}^{+}$and the positive-parity final state in the daughter even-even nucleus is denoted by $J_{f}^{+}$. In the closure approximation the intermediate energies $E_{k}$ in Eqs. (5.2)-(5.4) are replaced by a single energy $E$ and the summation over $k$ is replaced by a unit operator.

In general, for the near-resonant $0 \nu 2 \mathrm{EC}$ decay to the final $J_{f}^{+}=0^{+}, 1^{+}, 2^{+}$states the NMEs can be written in the QRPA framework in the form

$$
\begin{aligned}
M_{K}^{2 \mathrm{EC}}\left(0_{i}^{+} \rightarrow J_{f}^{+}\right)= & (-1)^{J_{f}} \sum_{J^{\pi}, k_{1}, k_{2}} \sum_{J_{1}, J^{\prime}, J^{\prime \prime}} \sum_{p p^{\prime} n n^{\prime}} \sqrt{\frac{\left[J^{\prime}\right]\left[J^{\prime \prime}\right]\left[J_{1}\right]}{\left[J_{f}\right]}}\left\{\begin{array}{ccc}
j_{n} & j_{p} & J \\
j_{n^{\prime}} & j_{p^{\prime}} & J_{1} \\
J^{\prime \prime} & J^{\prime} & J_{f}
\end{array}\right\}\left(n n^{\prime}: J^{\prime \prime}\left\|\mathcal{O}_{K}^{\left(J_{f}\right)}\right\| p p^{\prime}: J^{\prime}\right) \\
& \times\left(J_{f}^{+}\left\|\left[c_{n^{\prime}}^{\dagger}, \tilde{c}_{p^{\prime}}\right]_{J_{1}}\right\| J_{k_{1}}^{\pi}\right)\left\langle J_{k_{1}}^{\pi} \mid J_{k_{2}}^{\pi}\right\rangle\left(J_{k_{2}}^{\pi}\left\|\left[c_{n}^{\dagger} \tilde{c}_{p}\right]_{J}\right\| 0_{i}^{+}\right),
\end{aligned}
$$

where $[x]=2 x+1$ and $k_{1}$ and $k_{2}$ label the different QRPA solutions for a given multipole $J^{\pi}$, stemming from the parent and daughter nuclei of the near-resonant 0 2 EC decay. The operators $\mathcal{O}_{K}^{\left(0_{f}\right)}$ for the $0^{+}$final states in the reduced two-particle matrix element denote the Fermi $(K=\mathrm{F})$, Gamow-Teller $(K=\mathrm{GT})$, and tensor $(K=\mathrm{T})$ parts of the double-beta operator, given in Eqs. (5.2)-(5.4). In all discussed theory frameworks the two-particle matrix element also contains the appropriate short-range correlations, higher-order nucleonic weak currents, and nucleon form factors, as given by Šimkovic et al. (1999). The last line of Eq. (5.6) contains the one-body transition densities between the initial $\left(0_{i}^{+}\right)$ and final $\left(0_{f}^{+}\right)$ground states and the intermediate states $J_{k}^{\pi}$, and they can be obtained in the QRPA framework as discussed later. The term between the one-body transition densities is the overlap between the two sets of intermediate states emerging from the two QRPA calculations based on the parent and daughter even-even ground states and its expression for the spherical nuclei was given by Suhonen (2012a) and for deformed nuclei by Šimkovic, Pacearescu, and Faessler (2004).

Here we note that typically only the $J_{f}^{+}=0^{+}$final states have been considered in the near-resonant $0 \nu 2 \mathrm{EC}$-decay calculations, as given by Eqs. (5.2)-(5.4) and the $J_{f}=0$ special case of Eq. (5.6). The simplest procedure to reach the positive-parity $J_{f}^{+}=1^{+}, 2^{+}$states is to use a generalized GT-type operator

$$
\begin{aligned}
& M_{\mathrm{GT}}^{2 \mathrm{EC}}\left(J_{f}^{+}=1^{+}, 2^{+}\right) \\
& \quad=\sum_{k}\left(J_{f}^{+}\left\|\sum_{m n} h_{\mathrm{GT}}\left(r_{m n}, E_{k}\right)\left[\boldsymbol{\sigma}_{m} \boldsymbol{\sigma}_{n}\right]_{J_{f}}\right\| 0_{i}^{+}\right),
\end{aligned}
$$

together with Eq. (5.6). In Sec. V.C we compute this NME for several cases of interest in an approximate way, avoiding the vast complications involved in the use of detailed nuclear wave functions for high-excited states in heavy daughter nuclei of the near-resonant $0 \nu 2 \mathrm{EC}$ processes. Furthermore, to reach the negative-parity states $J_{f}^{-}=0^{-}, 1^{-}, 2^{-}$one would need more complex nuclear transition operators, and these have not yet been thoroughly examined (Vergados, 2011). In this review we then skip the estimation of the order of magnitude of the related NMEs.

Here we remark that Cirigliano et al. (2018b, 2019) found that in addition to the long-range NME (5.1) there is a notable contribution from a short-range operator affecting the Fermi part of the NME. According to preliminary studies in these works for extremely light nuclei the value of the NME of the neutrinoless double-beta decay could be changed considerably by the inclusion of the new short-range term. It remains to be seen how strong the effect is for the medium-heavy and heavy nuclei that actually double beta decay.

\section{Multiple-commutator model}

The nuclear states of odd-odd nuclei can be described within the spherical proton-neutron quasiparticle random-phase 
approximation (pnQRPA) framework. The solution of the pnQRPA equations can be written as [see Suhonen (2007)]

$$
\begin{aligned}
|\omega M\rangle & =q^{\dagger}(\omega, M)|\mathrm{QRPA}\rangle \\
& =\sum_{p n}\left(X_{p n}^{\omega}\left[a_{p}^{\dagger} a_{n}^{\dagger}\right]_{J M}-Y_{p n}^{\omega}\left[a_{p}^{\dagger} a_{n}^{\dagger}\right]_{J M}^{\dagger}\right)|\mathrm{QRPA}\rangle,
\end{aligned}
$$

where the shorthand $\omega=J_{k}^{\pi}$ for the $k$ th intermediate state of spin parity $J^{\pi}$ is used. Here $|\mathrm{QRPA}\rangle$ is the QRPA ground state and the operator $a_{p}^{\dagger}$ creates a proton quasiparticle on the singleparticle orbital $p=\left(n_{p}, l_{p}, j_{p}\right)$, where $n$ is the principal, $l$ is the orbital angular momentum, and $j$ is the total angularmomentum quantum number. The operator $a_{p}$ is the corresponding annihilation operator and a similar definition applies for the neutrons $n$. The single-particle orbitals are obtained by solving the Schrödinger equation for a spherical Woods-Saxon mean-field potential (Suhonen, 2007). By using this wave function one can obtain the transition densities

$$
\begin{aligned}
& \left(0_{\mathrm{gS}}^{+}\left\|\left[c_{n^{\prime}}^{\dagger} \tilde{c}_{p^{\prime}}\right]_{J}\right\| J_{k_{1}}^{\pi}\right)=\sqrt{[J]}\left[v_{n^{\prime}} u_{p^{\prime}} X_{p^{\prime} n^{\prime}}^{J^{\pi} k_{1}}+u_{n^{\prime}} v_{p^{\prime}} Y_{p^{\prime} n^{\prime}}^{J^{\pi} k_{1}}\right], \\
& \left(J_{k_{2}}^{\pi}\left\|\left[c_{n}^{\dagger} \tilde{c}_{p}\right]_{J}\right\| 0_{i}^{+}\right)=\sqrt{[J]}\left[\tilde{u}_{n} \tilde{v}_{p} \tilde{X}_{p n}^{J^{\pi} k_{2}}+\tilde{v}_{n} \tilde{u}_{p} \tilde{Y}_{p n}^{J^{\pi} k_{2}}\right],
\end{aligned}
$$

where $v(\tilde{v})$ and $u(\tilde{u})$ correspond to the BCS occupation and vacancy amplitudes of the final (initial) even-even nucleus. The amplitudes $X$ and $Y(\tilde{X}$ and $\tilde{Y})$ come from the pnQRPA calculation starting with the final (initial) nucleus of the doublebeta decay. Here the initial and final states of the near-resonant $0 \nu 2 \mathrm{EC}$ decay are assumed to be the ground states of the eveneven mother and daughter nuclei.

The $n$th excited $\omega_{f}=I_{n}^{\pi}$ state, where $I$ is the angular momentum of the state, in the even-even daughter nucleus is described in the QRPA formalism, and the corresponding wave function can be presented as [see Suhonen (2007)]

$$
\begin{aligned}
\left|I_{n}^{\pi} M_{I}\right\rangle & =Q^{\dagger}\left(I_{n}^{\pi}, M_{I}\right)|\mathrm{QRPA}\rangle \\
& =\sum_{a \leq b}\left[X_{a b}^{\omega_{f}} A_{a b}^{\dagger}\left(I M_{I}\right)-Y_{a b}^{\omega_{f}} \tilde{A}_{a b}\left(I M_{I}\right)\right]|\mathrm{QRPA}\rangle,
\end{aligned}
$$

where the normalized two-quasiparticle operators are defined as

$$
\begin{aligned}
A_{a b}^{\dagger}\left(I M_{I}\right) & =\mathcal{N}_{a b}(I)\left[a_{a}^{\dagger} a_{b}^{\dagger}\right]_{I M_{I}}, \\
\mathcal{N}_{a b}(I) & =\frac{\sqrt{1+\delta_{a b}(-1)^{I}}}{1+\delta_{a b}}
\end{aligned}
$$

for any state of angular momentum $I$ in the even-even nucleus. We denote here $\tilde{A}_{a b}\left(I M_{I}\right) \equiv(-1)^{I+M_{I}} A_{a b}\left(I,-M_{I}\right)$. Here $a$ and $b$ denote the quantum numbers of a single-particle orbital in a spherical nuclear mean field, including the number of nodes $n$ (principal quantum number) and the orbital $(l)$ and total $(j)$ angular momenta. We note that the summation over $a \leq b$ guarantees that there is no double counting of twoquasiparticle configurations and that this along with the normalized operators (5.12) guarantees that the wave function will be properly normalized with the normalization condition (Suhonen, 2007)

$$
\sum_{a \leq b}\left(\left|X_{a b}^{\omega_{f}}\right|^{2}-\left|Y_{a b}^{\omega_{f}}\right|^{2}\right)=1
$$

The creation operator $Q^{\dagger}\left(I_{n}^{\pi}, M_{I}\right)$ of Eq. (5.11) is usually called the creation operator for a QRPA phonon.

For calculational convenience it is preferable to go from the restricted sum of Eq. (5.11) to a nonrestricted (free) one by introducing the correspondence

$$
\begin{aligned}
\left|I_{n}^{\pi} M_{I}\right\rangle & =\bar{Q}^{\dagger}\left(I_{n}^{\pi}, M_{I}\right)|\mathrm{QRPA}\rangle \\
& =\sum_{a b}\left[\bar{X}_{a b}^{\omega_{f}} \bar{A}_{a b}^{\dagger}\left(I M_{I}\right)-\bar{Y}_{a b}^{\omega_{f}} \tilde{\bar{A}}_{a b}\left(I M_{I}\right)\right]|\mathrm{QRPA}\rangle,
\end{aligned}
$$

where the barred two-quasiparticle operators are those in Eq. (5.12) without the normalizer $\mathcal{N}_{a b}(I)$. Then the normalization condition becomes

$$
\sum_{a b}\left(\left|\bar{X}_{a b}^{\omega_{f}}\right|^{2}-\left|\bar{Y}_{a b}^{\omega_{f}}\right|^{2}\right)=\frac{1}{2}
$$

At the same time the two kinds of $X$ and $Y$ amplitudes are related by

$\bar{X}_{a b}^{\omega_{f}}=\frac{\sqrt{1+\delta_{a b}}}{2} X_{a b}^{\omega_{f}}, \quad \bar{Y}_{a b}^{\omega_{f}}=\frac{\sqrt{1+\delta_{a b}}}{2} Y_{a b}^{\omega_{f}}, \quad a \leq b$,

for any $\omega_{f}=I_{n}^{\pi}$. The barred amplitudes are symmetrized ones and possess convenient symmetry relations to generate amplitudes with $a>b$ :

$\bar{X}_{b a}^{\omega_{f}}=(-1)^{j_{a}+j_{b}+J+1} \bar{X}_{a b}^{\omega_{f}}, \quad \bar{Y}_{b a}^{\omega_{f}}=(-1)^{j_{a}+j_{b}+J+1} \bar{Y}_{a b}^{\omega_{f}}$.

In the MCM, originally introduced by Suhonen (1993), the one-body transition densities corresponding to a transition from the intermediate $|\omega M\rangle$ state (5.8) of the odd-odd nucleus to the final one-phonon state (5.15) of the even-even daughter nucleus are calculated by first writing the transition densities as ground-state-averaged multiple commutators and then applying the quasiboson approximation (Suhonen, 2007) by replacing the QRPA vacuum with the BCS vacuum when taking the ground-state average. The averaged multiple commutators then become

$$
\begin{aligned}
& \left\langle\omega_{f} M_{I}\left|\beta_{L \mu}^{+}(n p)\right| \omega M\right\rangle \\
& \quad \approx\left\langle\mathrm{BCS}\left|\left[\left[\bar{Q}\left(\omega_{f}, M_{I}\right), \beta_{L \mu}^{+}(n p)\right], q^{\dagger}(\omega, M)\right]\right| \mathrm{BCS}\right\rangle,
\end{aligned}
$$

where $|\mathrm{BCS}\rangle$ is the BCS ground state and we denote the $\beta^{+}$ type of EC operator by

$$
\beta_{L \mu}^{+}(n p) \equiv\left[c_{n}^{\dagger} \tilde{c}_{p}\right]_{L \mu},
$$

with $c_{n}^{\dagger}$ creating a neutron on orbital $n$ and $\tilde{c}_{p}$ annihilating a proton on orbital $p$. Using the Wigner-Eckart theorem (Suhonen, 2007) one can convert Eq. (5.19) to the reduced transition density 


$$
\begin{aligned}
\left(\omega_{f}\left\|\left[c_{n}^{\dagger} \tilde{c}_{p}\right]_{L}\right\| \omega\right)= & 2 \sqrt{[I][L][J]}(-1)^{I+L} \times \sum_{n^{\prime}}(-1)^{j_{p}+j_{n^{\prime}}}\left(\bar{X}_{n^{\prime} n}^{\omega_{f}} X_{n^{\prime} p}^{\omega} u_{n} u_{p}-\bar{Y}_{n^{\prime} n}^{\omega_{f}} Y_{n^{\prime} p}^{\omega} v_{n} v_{p}\right)\left\{\begin{array}{ccc}
J & L & I \\
j_{n} & j_{n^{\prime}} & j_{p}
\end{array}\right\} \\
& +2 \sqrt{[I][L][J]}(-1)^{I+J} \times \sum_{p^{\prime}}(-1)^{j_{n}+j_{p}}\left(-\bar{X}_{p^{\prime} p}^{\omega_{f}} X_{n p^{\prime}}^{\omega} v_{n} v_{p}+\bar{Y}_{p^{\prime} p}^{\omega_{f}} Y_{n p^{\prime}}^{\omega} u_{n} u_{p}\right)\left\{\begin{array}{lll}
J & L & I \\
j_{p} & j_{p^{\prime}} & j_{n}
\end{array}\right\},
\end{aligned}
$$

where the $v$ and $u$ factors are the occupation and vacancy amplitudes of the BCS ground state (Suhonen, 2007). The transition density (5.21) can be used to compute the connection of the near-resonant $0 \nu 2 \mathrm{EC}$ intermediate states to the final resonant excited state $\omega_{f}$ of Eq. (5.15).

The MCM method has close connection with the bosonexpansion method described by Raduta, Faessler, and Stoica (1991), Raduta et al. (1991), and Raduta and Suhonen (1996).

\section{Deformed quasiparticle random-phase approximation}

The near-resonant $0 \nu 2 \mathrm{EC}$ NMEs for axially symmetric well-deformed nuclei can be calculated in the adiabatic BohrMottelson approximation in the intrinsic coordinate system of a rotating nucleus. The nuclear excitations are characterized by the parity $\pi$ and the quantum number $K$ that is associated with the projection of the total angular momentum $J$ of the nucleus onto the intrinsic symmetry axis. Then the $k$ th intrinsic state of projection parity $K^{\pi},\left|K^{\pi}, k\right\rangle$, can be generated by the deformed QRPA approach (Fang et al., 2011) in a way analogous to Eq. (5.8):

$$
\begin{aligned}
\left|K^{\pi} k\right\rangle & =q^{\dagger}\left(K^{\pi}, k\right)\left|0_{\mathrm{gs}}^{+}\right\rangle \\
& =\sum_{p n}\left(X_{p n, K}^{k} a_{p}^{\dagger} a_{\bar{n}}^{\dagger}-Y_{p n, K}^{k} a_{\bar{p}} a_{n}\right)\left|0_{\mathrm{gs}}^{+}\right\rangle,
\end{aligned}
$$

where for the quasiparticle operators $a_{\bar{p}}\left(a_{\bar{n}}\right) \bar{p}(\bar{n})$ denotes the time-reversed proton (neutron) orbital. The quasiparticle pairs in Eq. (5.22) obey the selection rules $\Omega_{p}-\Omega_{n}=K$ and $\pi_{p} \pi_{n}=\pi$, where the involved parities are those of the singleparticle orbitals and $\Omega$ denotes the projection of the total single-particle angular momentum $j$ on the intrinsic symmetry axis. The state $\left|0_{\mathrm{gs}}^{+}\right\rangle$denotes the vacuum of the deformed QRPA. The single-particle states are obtained by solving the Schrödinger equation for a deformed axially symmetric Woods-Saxon mean-field potential (Yousef et al., 2009). In the deformed QRPA approach the deformed calculation is transformed to a spherical QRPA framework by first decomposing the deformed Woods-Saxon wave functions into deformed harmonic-oscillator (HO) wave functions. These, in turn, are decomposed into spherical $\mathrm{HO}$ wave functions (Yousef et al., 2009). This also enables the use of realistic oneboson-exchange nucleon-nucleon potentials in the many-body calculations (Yousef et al., 2009).

The one-body transition densities (5.9) and (5.10) of the spherical QRPA are now replaced by the corresponding transition densities of the deformed QRPA

$$
\left\langle 0_{\mathrm{gs}}^{+}\left|c_{n^{\prime}}^{\dagger} \tilde{c}_{p^{\prime}}\right| K^{\pi} k_{1}\right\rangle=v_{n^{\prime}} u_{p^{\prime}} X_{p^{\prime} n^{\prime}, K^{\pi}}^{k_{1}}+u_{n^{\prime}} v_{p^{\prime}} Y_{p^{\prime} n^{\prime}, K^{\pi}}^{k_{1}},
$$

$$
\left\langle K^{\pi} k_{2}\left|c_{n}^{\dagger} \tilde{c}_{p}\right| 0_{i}^{+}\right\rangle=\tilde{u}_{n} \tilde{v}_{p} \tilde{X}_{p n, K^{\pi}}^{k_{2}}+\tilde{v}_{n} \tilde{u}_{p} \tilde{Y}_{p n, K^{\pi}}^{k_{2}} .
$$

These transition densities are the ones used to compute the near-resonant $0 \nu 2 \mathrm{EC}$ NMEs of the decays of ${ }^{152} \mathrm{Gd},{ }^{164} \mathrm{Er}$, and ${ }^{180} \mathrm{~W}$ in Fang et al. (2012).

\section{Microscopic interacting boson model}

The interacting boson model (IBM) is a theory framework based on $s$ and $d$ bosons that correspond to collective nucleon pairs coupled to angular momenta and parities $0^{+}$and $2^{+}$, respectively. An extension of the IBM is the microscopic IBM (IBM-2) where the protons and neutrons form separate proton and neutron bosons. The IBM-2 is in a way a phenomenological version of the nuclear shell model, containing the seniority aspect and the restriction to one magic shell in terms of the single-particle model space. The Hamiltonian and the transition operators are constructed from the $s$ and $d$ bosons as lowest-order boson expansions with coupling coefficients to be determined by fits to experimental data on low-lying energy levels and E2 $\gamma$ transitions associated with the $s$ and $d$ bosons, but the fitting does not use the spin or isovector data available from the $\beta$ decays. One can also relate the bosons to the underlying fermion model space through a mapping procedure (Otsuka, Arima, and Iachello, 1978; Otsuka, 1996).

The microscopic IBM can be extended to include highermultipole bosons, like $g$ bosons, as well. Further extension concerns the description of odd- $A$ nuclei using the microscopic interacting boson-fermion model (IBFM-2) (Iachello and Van Isacker, 1991). The IBM concept can also be used to describe odd-odd nuclei by using the interacting bosonfermion-fermion model (IBFFM) and its proton-neutron variant, the proton-neutron IBFFM (IBFFM-2) (Brant and Paar, 1988). Here problems arise from the interactions between the bosons and the one or two extra fermions in the Hamiltonian, and from the transition operators containing a large number of phenomenological parameters to be determined in some meaningful way. While IBM-2 has often been used to calculate the $0 \nu 2 \beta$ properties of nuclei, the IBFFM-2 has not. IBM-2 calculations have to be done using the closure approximation since it does not contain the spinisospin degree of freedom needed to access the intermediate odd-odd nucleus of the $0 \nu 2 \beta$ decay, particularly in the context of the near-resonant $0 \nu 2 \mathrm{EC}$ decays.

\section{Energy-density functional method}

The EDF method is a mean-field-based method that uses closure approximation to compute the near-resonant $0 \nu 2 \mathrm{EC}$ 
NMEs, and thus is well suited for $0 \nu 2 \mathrm{EC}$ transitions between two ground states, as in the cases of the near-resonant $0 \nu 2 \mathrm{EC}$ decays of ${ }^{152} \mathrm{Gd},{ }^{164} \mathrm{Er}$, and ${ }^{180} \mathrm{~W}$ treated by Rodríguez and Martínez-Pinedo (2012). In this theory framework (Rodríguez and Martínez-Pinedo, 2010) density functionals based on the Gogny D1S functional (Berger, Girod, and Gogny, 1984) and D1M functional (Goriely et al., 2009) in large singleparticle bases (11 major oscillator shells) are used. Both the particle-number and angular-momentum projections are performed before the variation for the mother and daughter nuclei, and configuration mixing is taken into account using the generator coordinate method (Ring and Schuck, 1980). Hence, in the EDF the initial and final ground states can be written as

$$
\left|0_{\mathrm{gs}}^{+}\right\rangle=\sum_{\beta_{2}} g_{\beta_{2}} P^{J=0} P^{N} P^{Z}\left|\Phi_{\beta_{2}}\right\rangle,
$$

where $P^{N}\left(P^{Z}\right)$ is the projection operator for a given neutron (proton) number and $P^{J=0}$ is the projection operator for zero total angular momentum. The intrinsic axially symmetric Hartree-Fock-Bogoliubov wave functions $\left|\Phi_{\beta_{2}}\right\rangle$ are solutions to the variation equations after particle-number-projection constrained to a given value of the axial quadrupole deformation $\beta_{2}$. The shape-mixing coefficients $g_{\beta_{2}}$ are found by solving the Hill-Wheeler-Griffin equation (Ring and Schuck, 1980).

\section{Decays of nuclides with the calculated nuclear matrix elements}

The order of magnitude of the NME (5.7) can be estimated by constructing a generic single-quasiparticle-type NME (qp-NME) describing the conversion of a proton pair to a neutron pair at the nuclear proton and neutron Fermi surfaces. This NME picks the essential features of the transition since most action is concentrated at the Fermi surfaces. The detailed quasiparticle properties at the Fermi surfaces can be obtained from a BCS calculation using the Woods-Saxon mean-field single-particle energies (Bohr and Mottelson, 1969). In this simple estimation the collective effects are not taken into account. These collective effects can be important for $0 \nu 2 \mathrm{EC}$ transitions to the lowest-lying $0^{+}$or $2^{+}$states. However, for the $J_{f}=0^{+}, 2^{+}$states at energies satisfying the resonance condition of the nearresonant $0 \nu 2 \mathrm{EC}$ decay the collective effects are not as important. In fact, at these energies the many-body wave functions can vary strongly from one state to the next, sometimes causing coherent enhancements or incoherent cancellations. A qp-NME is a kind of average between these two extremes and thus suitable for the role of a generic NME in this case.

A plausible simplification of the NME (5.6) is to consider the conversion of an angular-momentum-zero-coupled proton pair to an angular-momentum- $J_{f}$-coupled neutron pair at the nuclear Fermi surface. The zero-coupled proton pairs are the most important contributors to the NMEs of the ordinary $0 \nu 2 \beta$ decay (Hyvärinen and Suhonen, 2015), so this is a good simplifying approximation. Considering the $1^{+}$type of intermediate states the typical ones and taking $J_{1}=1$ for simplicity leads to the following simplified expression for the NME (5.6):

$$
\begin{aligned}
M_{K}^{2 \mathrm{EC}}\left(0_{i}^{+} \rightarrow J_{f}^{+}\right) \approx & \sqrt{\frac{3}{\left[J_{f}\right]\left[j_{p}\right]}}(-1)^{j_{p}+j_{n}+1}\left\{\begin{array}{ccc}
1 & j_{n} & j_{p} \\
j_{n} & 1 & J_{f}
\end{array}\right\} \\
& \times\left(n n: J_{f}\left\|h\left(r_{12}\right)\left[\boldsymbol{\sigma}_{1} \boldsymbol{\sigma}_{2}\right]_{J_{f}}\right\| p p: 0\right) \\
& \times\left(J_{f}^{+}\left\|\left[c_{n}^{\dagger} \tilde{c}_{p}\right]_{1}\right\| 1^{+}\right)\left(1^{+}\left\|\left[c_{n}^{\dagger} \tilde{c}_{p}\right]_{1}\right\| 0_{i}^{+}\right),
\end{aligned}
$$

where the neutrino potential can be simplified to a Coulombtype potential

$$
h\left(r_{12}\right)=\frac{2 R_{A}}{\pi} \frac{1}{r_{12}}
$$

by taking only the leading contribution (Hyvärinen and Suhonen, 2015) to the potential and approximating the difference of the intermediate energy and the average of the parent and daughter masses as zero, which is a rather good approximation for the ground state of the intermediate nucleus. Here $R_{A}=1.2 A^{1 / 3} \mathrm{fm}$ is the nuclear radius for the nucleus of mass $A$. To proceed further one has to convert the two-body NME to the center of mass and relative coordinates for the computation of the associated radial integral of the simplified neutrino potential (5.27). This can be achieved by the use of the Moshinsky brackets $M_{\lambda}$, first introduced by Moshinsky (1959); see Suhonen (2012a) for more details.

Implementing the Moshinsky brackets and working out the angular-momentum algebra result in a rather simple compact expression for the two-body NME in Eq. (5.26):

$$
\begin{aligned}
\left(n n: J_{f}\left\|h\left(r_{12}\right)\left[\boldsymbol{\sigma}_{1} \boldsymbol{\sigma}_{2}\right]_{J_{f}}\right\| p p: 0\right)= & 6 \sqrt{\left[J_{f}\right]\left[j_{p}\right]}\left[j_{n}\right] \sum_{S=0,1} G_{J_{f}}^{p n}(S) \\
& \times \sum_{n n^{\prime} l N L} M_{S}\left(n^{\prime} l N L ; n_{n} l_{n} n_{n} l_{n}\right) \\
& \times M_{S}\left(\mathrm{nlNL} ; n_{p} l_{p} n_{p} l_{p}\right) I_{n^{\prime} n l}
\end{aligned}
$$

where $n_{n}$ and $l_{n}$ are the principal and orbital angularmomentum quantum numbers for the orbital occupied by the final neutrons and $n_{p}$ and $l_{p}$ are the corresponding quantum numbers for the initial protons. The quantities $M_{S}\left(n^{\prime} l N L ; n_{n} l_{n} n_{n} l_{n}\right)=\left\langle n^{\prime} l, N L, S \mid n_{n} l_{n}, n_{n} l_{n}, S\right\rangle$ are the Moshinsky brackets and the sum over the quantum numbers $n$ and $n^{\prime}, l$ refers to a sum over the principal and orbital angular-momentum quantum numbers of the relative motion, and $N$ and $L$ symbolize the principal and orbital angularmomentum quantum numbers associated with the center-ofmass coordinate. The sum over $S$ denotes a sum over the possible total spins. The geometric factor can be simplified to 


$$
\begin{aligned}
G_{J_{f}}^{p n}(S)= & {[S] \sum_{S^{\prime}}\left[S^{\prime}\right](-1)^{S^{\prime}+J_{f}+l_{p}+j_{p}+1 / 2}\left\{\begin{array}{lll}
l_{p} & l_{p} & S \\
\frac{1}{2} & \frac{1}{2} & j_{p}
\end{array}\right\} } \\
& \times\left\{\begin{array}{ccc}
l_{n} & \frac{1}{2} & j_{n} \\
l_{n} & \frac{1}{2} & j_{n} \\
S & S^{\prime} & J_{f}
\end{array}\right\}\left\{\begin{array}{ccc}
\frac{1}{2} & \frac{1}{2} & 1 \\
\frac{1}{2} & \frac{1}{2} & 1 \\
S^{\prime} & S & J_{f}
\end{array}\right\},
\end{aligned}
$$

and the Coulomb-type integral reads

$$
I_{n^{\prime} n l}=\int_{0}^{\infty} g_{n^{\prime} l}(r) h(r) g_{n l}(r) r^{2} d r,
$$

where $g_{n l}(r)$ are the radial functions of the three-dimensional harmonic oscillator and $h(r)$ is the simplified neutrino potential (5.27).

The one-body transition densities involved in Eq. (5.26) can be obtained from Eqs. (5.10) and (5.21). In the quasiparticle approximation the $Y$ amplitudes vanish and for the involved quasiparticle transitions the $X$ factors are set to unity. Then one finds that

$$
\begin{aligned}
& \left(J_{f}^{+}\left\|\left[c_{n}^{\dagger} \tilde{c}_{p}\right]_{1}\right\| 1^{+}\right)\left(1^{+}\left\|\left[c_{n}^{\dagger} \tilde{c}_{p}\right]_{1}\right\| 0_{i}^{+}\right) \\
& \quad \approx 3 \sqrt{6\left[J_{f}\right]}(-1)^{J_{f}+1}\left\{\begin{array}{ccc}
1 & 1 & J_{f} \\
\frac{9}{2} & \frac{9}{2} & \frac{11}{2}
\end{array}\right\} u_{n} u_{p} \tilde{u}_{n} \tilde{v}_{p},
\end{aligned}
$$

where the occupation and vacancy amplitudes are obtained from BCS calculations in the involved nuclei. The qp-NMEs calculated by using the simplified formalism of Eqs. (5.26)-(5.29) are displayed in Table IV. In the $A=$ 148-154 region the proton-to-neutron single-quasiparticle transition is $\pi 0 h_{11 / 2} \rightarrow \nu 0 h_{9 / 2}$ and for $A=194,202$ the transition is $\pi 0 h_{11 / 2} \rightarrow \nu 0 i_{13 / 2}$.

\section{Summary}

To conclude, Table IV shows that the magnitudes of the computed $0 v 2 \mathrm{EC}$ NMEs for different theory frameworks can vary quite strongly for nuclei with $A \geq 152$. These nuclei are deformed and thus rather challenging from the nuclearstructure point of view. For these nuclei it is preferable to apply a nuclear-theory framework that naturally contains the deformation degree of freedom, namely, the IBM-2, dQRPA, and EDF frameworks. However, as seen in Table IV, the computed NMEs show that there are significant differences between the results obtained in the different computational formalisms. The reason for these differences is not obvious and is already well recognized in the case of the $0 \nu 2 \beta^{-}$NMEs, as shown in a NME compilation by Engel and Menxéndez (2017). Since none of these theory frameworks can systematically access the uncertainties of the calculations, it is hard to make a judicious choice between the different NMEs in terms of reliability. This conclusion is valid for both the $0 \nu 2 \beta^{-}$and $0 \nu 2 \mathrm{EC}$ decay processes. Only further studies and comparisons between these theory frameworks could shed light on this situation and lead to consistent values of the NMEs for deformed heavy nuclei.

Another conspicuous feature of Table IV is that the nuclear shell model (NSM), standardly used to compute the $0 \nu 2 \beta^{-}$
NMEs, does not contribute to the calculations of the $0 \nu 2 \mathrm{EC}$ NMEs. The reason for this is twofold: on the one hand, for the nuclei ${ }^{152} \mathrm{Gd},{ }^{156} \mathrm{Dy},{ }^{164} \mathrm{Er}$, and ${ }^{180} \mathrm{~W}$ the $2 \nu \mathrm{EC}$ transition is ground state to ground state and thus accessible, in principle, to the NSM. These nuclei are heavy highly deformed nuclei and the NSM simply does not have the necessary singleparticle valence space to treat these decays. On the other hand, for the lighter, nearly spherical nuclei, the NSM is easier to install in terms of single-particle spaces, but the fact that the resonant states in the daughter nuclei are highly excited excludes a reasonable description of the corresponding wave functions by the NSM.

\section{STATUS OF EXPERIMENTAL SEARCHES}

\section{A. Experimental studies of 2EC processes}

The efforts of experimentalists have mainly concentrated on the search for neutrinoless double-beta decay with emission of two electrons $\left(2 \beta^{-}\right)$where limits on the half-lives of $T_{1 / 2}>$ $10^{24}-10^{26} \mathrm{yr}$ were obtained. ${ }^{2}$ The most sensitive $0 \nu 2 \beta^{-}$ experiments provide limits on the effective Majorana mass of the electron neutrino of approximately $\left|m_{\beta \beta}\right|<0.1-0.7 \mathrm{eV}$. The uncertainties in $m_{\beta \beta}$ are related to the uncertainties inherent in the nuclear-structure model calculations of NMEs and the in-medium modifications of the axial-vector coupling $g_{A}$.

The sensitivity of the experiments in search for double-betaplus processes such as $2 \mathrm{EC}$, electron capture with positron emission $\left(\mathrm{EC} \beta^{+}\right)$, and double-positron emission $\left(2 \beta^{+}\right)$is substantially lower [see Tretyak and Zdesenko (1995) and Tretyak and Zdesenko (2002) and the references in Table $\mathrm{V}^{3}$ ]. The most sensitive experiments give limits on double-betaplus processes of approximately $\lim T_{1 / 2} \sim 10^{21}-10^{22} \mathrm{yr}$ (for ${ }^{36} \mathrm{Ar},{ }^{40} \mathrm{Ca},{ }^{58} \mathrm{Ni},{ }^{64} \mathrm{Zn},{ }^{78} \mathrm{Kr},{ }^{96} \mathrm{Ru},{ }^{106} \mathrm{Cd},{ }^{112} \mathrm{Sn},{ }^{120} \mathrm{Te},{ }^{124} \mathrm{Xe}$, ${ }^{126} \mathrm{Xe},{ }^{130} \mathrm{Ba}$, and $\left.{ }^{132} \mathrm{Ba}\right)$. We note that until recently there were only indications, even for the allowed two-neutrino doubleelectron capture. However, the XENON Collaboration (2019) claimed to have observed two-neutrino double-electron capture in ${ }^{124} \mathrm{Xe}$ with a half-life of $(1.8 \pm 0.5) \times 10^{22} \mathrm{yr}$. The result of the XENON1t data analysis in the region of interest for $2 \nu 2 \mathrm{EC}$ in ${ }^{124} \mathrm{Xe}$ is shown in Fig. 10. Doublebeta decay of ${ }^{130} \mathrm{Ba}$ was measured using the geochemical method; the half-life is $T_{1 / 2}=(2.2 \pm 0.5) \times 10^{21} \mathrm{yr}$ as obtained by Meshik et al. (2001), and $T_{1 / 2}=(6.0 \pm 1.1) \times 10^{20} \mathrm{yr}$ as obtained by Pujol et al. (2009). An indication

\footnotetext{
${ }^{2}$ See Tretyak and Zdesenko (1995, 2002), Elliott (2012), Giuliani and Poves (2012), Saakyan (2013), Cremonesi and Pavan (2014), Bilenky and Giunti (2015), Gómez-Cadenas and Martín-Albo (2015), Päs and Rodejohann (2015), Sarazin (2015), Dell'Oro et al. (2016), Vergados, Ejiri, and Šimkovic (2016), Barabash (2018), and Dolinski, Poon, and Rodejohann (2019), and the recent experimental results of Arnold et al. (2015), Gando et al. (2016), Agostini et al. (2018), Albert et al. (2018), Alduino et al. (2018a), and Azzolini et al. (2018).

${ }^{3}$ The energies $E^{*}$ and the values of $J^{\pi}$ of the excited nuclide levels in Table $\mathrm{V}$ were taken from the database of Brookhaven National Laboratory (http://www.nndc.bnl.gov/ensdf/).
} 
TABLE V. Experimental half-life limits of neutrinoless 2EC for transitions to the ground state (denoted as "g.s.") or to the excited level of the daughter nuclide with possible resonant enhancement. The mass differences between the mother and the daughter atoms $Q=M_{A, Z}-M_{A, Z-2}$ are from Wang et al. (2017); $\imath$ is the isotopic abundance of the nuclide of interest in the natural isotopic compositions of the elements (Meija et al., 2016). To check the resonance enhancement condition, the degeneracy parameter $\Delta=Q-E^{*}-\epsilon_{\alpha \beta}^{*}$ is shown, where $E^{*}=M_{A, Z-2}^{*}-$ $M_{A, Z-2}$ is the excitation energy of the daughter nuclide and $\epsilon_{\alpha \beta}^{*}=M_{A, Z-2}^{* *}-M_{A, Z-2}^{*}$ is the excitation energy of the atomic shell with the electron vacancies $\alpha$ and $\beta$ in the $K, L, M$, or $N$ orbits. The experimental limits of the ${ }^{54} \mathrm{Fe} \rightarrow{ }^{54} \mathrm{Cr}$ decay are at $68 \%$ confidence level (C.L.), and in other cases at $90 \%$ C.L. The deexcitation width of the electron shell of the daughter nuclides $\Gamma_{f}=\Gamma_{\alpha}+\Gamma_{\beta}$ [see Campbell and Papp (2001)] is shown in column 6 (orbits are indicated in the brackets). The resonance parameter $R_{f}=\Gamma_{f} /\left(\Delta^{2}+\Gamma_{f}^{2} / 4\right)$ normalized on the value for the $0 \nu 2 \mathrm{EC}$ decay ${ }^{54} \mathrm{Fe} \rightarrow{ }^{54} \mathrm{Cr}$ (g.s. to g.s.) is given in column 7 .

\begin{tabular}{|c|c|c|c|c|c|c|}
\hline $\begin{array}{l}\text { Transition } \\
Q(\mathrm{keV}) \\
l(\%) \\
\end{array}$ & $\begin{array}{l}\text { Decay channel, } \\
\text { level of daughter } \\
\text { nuclei }(\mathrm{keV})\end{array}$ & $\Delta(\mathrm{keV})$ & $\begin{array}{c}\text { Expt. } \\
\text { limit (yr) }\end{array}$ & $\begin{array}{l}\text { Experimental } \\
\text { technique } \\
\text { (reference) }\end{array}$ & $\Gamma_{f}(\mathrm{eV})$ & $R_{f}$ \\
\hline $\begin{array}{l}{ }^{36} \mathrm{Ar} \rightarrow{ }^{36} \mathrm{~S} \\
432.59(19) \\
0.3336(210)\end{array}$ & $K L, 0^{+}$g.s. & $427.65(19)$ & $\geq 3.6 \times 10^{21}$ & $\begin{array}{l}\text { HPGe } \gamma \text { spectrometry } \\
\quad \text { (Agostini } \text { et al., 2016) }\end{array}$ & $1.04(K K)$ & 1.2 \\
\hline $\begin{array}{l}{ }^{40} \mathrm{Ca} \rightarrow{ }^{40} \mathrm{Ar} \\
193.51(2) \\
96.941(156)\end{array}$ & $2 \mathrm{EC}, 0^{+}$g.s. & $187.10(2)$ & $\geq 1.4 \times 10^{22}$ & $\begin{array}{r}\mathrm{CaWO}_{4} \text { scint. bolometer } \\
\text { (Angloher et al., 2016) }\end{array}$ & $1.32(K K)$ & 8 \\
\hline $\begin{array}{r}{ }^{50} \mathrm{Cr} \rightarrow{ }^{50} \mathrm{Ti} \\
1169.6(5) \\
4.345(13)\end{array}$ & & $1159.7(5)$ & & & $1.78(K K)$ & 0.3 \\
\hline \multirow[t]{3}{*}{$\begin{array}{l}{ }^{54} \mathrm{Fe} \rightarrow{ }^{54} \mathrm{Cr} \\
680.3(4) \\
5.845(105)\end{array}$} & $K K, 0^{+}$g.s. & $668.3(4)$ & $\geq 4.4 \times 10^{20}$ & $\begin{array}{l}\text { HPGe } \gamma \text { spectrometry } \\
\text { (Bikit et al., 1998) }\end{array}$ & $2.04(K K)$ & 1 \\
\hline & $K L, 0^{+}$g.s. & & $\geq 4.1 \times 10^{20}$ & $\begin{array}{l}\text { HPGe } \gamma \text { spectrometry } \\
\text { (Bikit et al., 1998) }\end{array}$ & & \\
\hline & $L L, 0^{+}$g.s. & & $\geq 5.0 \times 10^{20}$ & $\begin{array}{l}\text { HPGe } \gamma \text { spectrometry } \\
\text { (Bikit et al., 1998) }\end{array}$ & & \\
\hline \multirow[t]{2}{*}{$\begin{array}{l}{ }^{58} \mathrm{Ni} \rightarrow{ }^{58} \mathrm{Fe} \\
1926.4(3) \\
\quad 68.0769(190)\end{array}$} & $K L, 0^{+}$g.s. & & $\geq 4.1 \times 10^{22}$ & $\begin{array}{l}\text { HPGe } \gamma \text { spectrometry } \\
\text { (Rukhadze et al., } \\
\text { 2020) }\end{array}$ & & \\
\hline & $K K, 2^{+} 1674.731(6)$ & $237.4(3)$ & & & $2.38(K K)$ & 9 \\
\hline $\begin{array}{r}{ }^{64} \mathrm{Zn} \rightarrow{ }^{64} \mathrm{Ni} \\
1094.9(7) \\
49.17(75)\end{array}$ & $2 \mathrm{EC}, 0^{+}$g.s. & $1075.6(7)$ & $\geq 3.2 \times 10^{20}$ & $\begin{array}{l}\mathrm{ZnWO}_{4} \text { scintillator } \\
\quad \text { (Belli, Bernabei et al., } \\
\text { 2011b) }\end{array}$ & & \\
\hline \multirow{9}{*}{$\begin{array}{l}{ }^{74} \mathrm{Se} \rightarrow{ }^{74} \mathrm{Ge} \\
1209.24(1) \\
0.86(3)\end{array}$} & $K K, 0^{+}$g.s. & & $\geq 4.8 \times 10^{19}$ & $\begin{array}{l}\text { HPGe } \gamma \text { spectrometry } \\
\quad \text { (Barabash } \text { et al., 2020) }\end{array}$ & & \\
\hline & $K L, 0^{+}$g.s. & & $\geq 3.5 \times 10^{19}$ & $\begin{array}{l}\text { HPGe } \gamma \text { spectrometry } \\
\quad \text { (Barabash } \text { et al., 2020) }\end{array}$ & & \\
\hline & & & $\geq 9.6 \times 10^{18}$ & $\begin{array}{l}\text { HPGe } \gamma \text { spectrometry } \\
\text { (Lehnert, Wester et al., } \\
\text { 2016) }\end{array}$ & & \\
\hline & $L L, 0^{+}$g.s. & & $\geq 6.5 \times 10^{19}$ & $\begin{array}{l}\text { HPGe } \gamma \text { spectrometry } \\
\quad(\text { Barabash } \text { et al., 2020) }\end{array}$ & & \\
\hline & & & $\geq 5.8 \times 10^{18}$ & $\begin{array}{l}\text { HPGe } \gamma \text { spectrometry } \\
\text { (Lehnert, Wester et al., } \\
\text { 2016) }\end{array}$ & & \\
\hline & $\begin{array}{l}L L, 2^{+} \\
1204.205(7)\end{array}$ & $(2.21-2.60) \pm 0.01$ & $\geq 1.1 \times 10^{19}$ & $\begin{array}{l}\text { HPGe } \gamma \text { spectrometry } \\
\quad(\text { Barabash } \text { et al., 2020) }\end{array}$ & $7.6\left(L_{1} L_{1}\right)$ & $3.4 \times 10^{5}$ \\
\hline & & & $\geq 4.3 \times 10^{19 \mathrm{a}}$ & $\begin{array}{l}\text { HPGe } \gamma \text { spectrometry } \\
\quad(\text { Frekers } \text { et al., 2011) }\end{array}$ & & \\
\hline & & & $\geq 1.5 \times 10^{19 \mathrm{~b}}$ & $\begin{array}{l}\text { HPGe } \gamma \text { spectrometry } \\
\text { (Ješkovský et al., } \\
\text { 2015) }\end{array}$ & & \\
\hline & & & $\geq 7.0 \times 10^{18}$ & $\begin{array}{l}\text { HPGe } \gamma \text { spectrometry } \\
\text { (Lehnert, Wester et al., } \\
\text { 2016) }\end{array}$ & & \\
\hline${ }^{78} \mathrm{Kr} \rightarrow{ }^{78} \mathrm{Se}$ & $K K, 0^{+}$g.s. & & $\geq 5.5 \times 10^{21}$ & Proportional counter & & \\
\hline
\end{tabular}


TABLE V. (Continued)

\begin{tabular}{|c|c|c|c|c|c|c|}
\hline $\begin{array}{l}\text { Transition } \\
Q(\mathrm{keV}) \\
\imath(\%) \\
\end{array}$ & $\begin{array}{l}\text { Decay channel, } \\
\text { level of daughter } \\
\text { nuclei }(\mathrm{keV})\end{array}$ & $\Delta(\mathrm{keV})$ & $\begin{array}{c}\text { Expt. } \\
\text { limit (yr) }\end{array}$ & $\begin{array}{l}\text { Experimental } \\
\text { technique } \\
\text { (reference) }\end{array}$ & $\Gamma_{f}(\mathrm{eV})$ & $R_{f}$ \\
\hline $\begin{array}{l}2847.67(26) \\
0.355(3)\end{array}$ & $L L,\left(2^{+}\right) 2838.49(7)$ & $(5.88-6.32) \pm 0.26$ & $\geq 5.4 \times 10^{21}$ & $\begin{array}{l}\text { filled with enriched } \\
{ }^{78} \mathrm{Kr}(99.8 \%) \\
\text { (Gavrilyuk et al., } \\
\text { 2013) }\end{array}$ & $7.6\left(L_{1} L_{1}\right)$ & $4.8 \times 10^{4}$ \\
\hline \multirow{4}{*}{$\begin{array}{l}{ }^{84} \mathrm{Sr} \rightarrow{ }^{84} \mathrm{Kr} \\
1789.8(12) \\
0.56(2)\end{array}$} & $K K, 0^{+}$g.s. & & $\geq 6.0 \times 10^{16}$ & $\begin{array}{l}\text { HPGe } \gamma \text { spectrometry } \\
\quad(\text { Belli et al., 2012a) }\end{array}$ & & \\
\hline & $K L, 0^{+}$g.s. & & $\geq 1.9 \times 10^{16}$ & $\begin{array}{l}\text { HPGe } \gamma \text { spectrometry } \\
\quad(\text { Belli et al., 2012a) }\end{array}$ & & \\
\hline & $L L, 0^{+}$g.s. & & $\geq 5.9 \times 10^{16}$ & $\begin{array}{l}\text { HPGe } \gamma \text { spectrometry } \\
\quad(\text { Belli et al., 2012a) }\end{array}$ & & \\
\hline & $K K, 2^{+} 881.615(3)$ & $879.5(12)$ & & & $5.4(K K)$ & 1.5 \\
\hline \multirow[t]{3}{*}{$\begin{array}{l}{ }^{92} \mathrm{Mo} \rightarrow{ }^{92} \mathrm{Zr} \\
1650.45(19) \\
14.649(106)\end{array}$} & $K K, 0^{+}$g.s. & & $\geq 6.8 \times 10^{19}$ & $\begin{array}{l}\mathrm{CaMoO}_{4} \text { scintillator in } \\
\text { coincidence } \\
\text { with HPGe detector } \\
\text { (Kang et al., 2013) }\end{array}$ & & \\
\hline & $K K, 0^{+} 1382.77(7)$ & $231.68(19)$ & & & $7.66(K K)$ & 31 \\
\hline & $K K, 4^{+} 1495.46(5)$ & $118.99(19)$ & & & $7.66(K K)$ & 118 \\
\hline \multirow{6}{*}{$\begin{array}{l}{ }^{96} \mathrm{Ru} \rightarrow{ }^{96} \mathrm{Mo} \\
2714.50(12) \\
5.54(14)\end{array}$} & $K K 0^{+}$g.s. & & $\geq 1.0 \times 10^{21}$ & $\begin{array}{l}\text { HPGe } \gamma \text { spectrometry } \\
\quad(\text { Belli et al., 2013b) }\end{array}$ & & \\
\hline & $K L 0^{+}$g.s. & & $\geq 2.3 \times 10^{20}$ & $\begin{array}{l}\text { HPGe } \gamma \text { spectrometry } \\
\quad(\text { Belli et al., 2013b) }\end{array}$ & & \\
\hline & $L L 0^{+}$g.s. & & $\geq 2.3 \times 10^{20}$ & $\begin{array}{l}\text { HPGe } \gamma \text { spectrometry } \\
\quad(\text { Belli } \text { et al., 2013b) }\end{array}$ & & \\
\hline & $K L, 2^{+} 2700.21(6)$ & $-(8.23-8.58) \pm 0.13$ & $\geq 2.0 \times 10^{20}$ & $\begin{array}{l}\text { HPGe } \gamma \text { spectrometry } \\
\quad(\text { Belli et al., 2013b) }\end{array}$ & $8.32\left(K L_{1}\right)$ & $2.5 \times 10^{4}$ \\
\hline & $L L, 2^{+} 2700.21(6)$ & $(8.56-9.25) \pm 0.13$ & & & $7.6\left(L_{1} L_{1}\right)$ & $2.3 \times 10^{4}$ \\
\hline & $L L, 2712.68(10)$ & $-(3.22-3.91) \pm 0.16$ & $\geq 3.6 \times 10^{20}$ & $\begin{array}{l}\text { HPGe } \gamma \text { spectrometry } \\
\quad(\text { Belli et al., 2013b) }\end{array}$ & $5.63 L_{1} L_{2}$ & $9.0 \times 10^{4}$ \\
\hline $\begin{array}{l}{ }^{102} \mathrm{Pd} \rightarrow{ }^{102} \mathrm{Ru} \\
1203.3(4) \\
1.02(1)\end{array}$ & $K K, 2^{+} 1103.047(13)$ & $56.0(4)$ & & & $10.7(K K)$ & 738 \\
\hline \multirow[t]{8}{*}{$\begin{array}{l}{ }^{106} \mathrm{Cd} \rightarrow{ }^{106} \mathrm{Pd} \\
2775.39(10) \\
1.245(22)\end{array}$} & $K K, 0^{+}$g.s. & & $\geq 1.0 \times 10^{21}$ & $\begin{array}{l}\text { Enr. }{ }^{106} \mathrm{CdWO}_{4} \\
\quad \text { scintillator }(\text { Belli } \\
\quad \text { et al., 2012b) }\end{array}$ & & \\
\hline & $K L, 0^{+}$g.s. & & $\geq 1.3 \times 10^{21}$ & $\begin{array}{l}\text { Enr. }{ }^{106} \mathrm{CdWO}_{4} \\
\quad \text { scintillator }{ }^{\mathrm{c}}(\text { Belli } \\
\quad \text { et al., 2020) }\end{array}$ & & \\
\hline & $L L, 0^{+}$g.s. & & $\geq 1.0 \times 10^{21}$ & $\begin{array}{l}\text { Enr. }{ }^{106} \mathrm{CdWO}_{4} \\
\quad \text { scintillator (Belli } \\
\quad \text { et al., 2012b) }\end{array}$ & & \\
\hline & $K K, 0^{+} 2624.40(5)$ & $102.29 \pm 0.11$ & & & $12.5(K K)$ & 260 \\
\hline & $K K,(1)^{+} 2705.30(8)$ & $21.39 \pm 0.13$ & & & $12.5(K K)$ & $5.9 \times 10^{3}$ \\
\hline & $K K, 2717.59(21)$ & $9.10 \pm 0.23$ & $\geq 2.9 \times 10^{21}$ & $\begin{array}{l}\text { Enr. }{ }^{106} \mathrm{CdWO}_{4} \\
\quad \text { scintillator }{ }^{\mathrm{c}}(\text { Belli } \\
\quad \text { et al., 2020) }\end{array}$ & $12.5(K K)$ & $3.3 \times 10^{4}$ \\
\hline & $K L, 4^{+} 2741.0(5)$ & $(6.4-6.9) \pm 0.5$ & $\geq 9.5 \times 10^{20}$ & $\begin{array}{l}\text { Enr. }{ }^{106} \mathrm{CdWO}_{4} \\
\quad \text { scintillator (Belli } \\
\quad \text { et al., 2012b) }\end{array}$ & $10.2\left(K L_{1}\right)$ & $5.3 \times 10^{4}$ \\
\hline & & & $\geq 1.7 \times 10^{20}$ & $\begin{array}{l}\text { HPGe } \gamma \text { spectrometry of } \\
\text { enriched }{ }^{106} \mathrm{Cd} \\
\quad \text { (Rukhadze et al., } \\
\text { 2011b) }\end{array}$ & & \\
\hline
\end{tabular}


TABLE V. (Continued)

\begin{tabular}{|c|c|c|c|c|c|c|}
\hline $\begin{array}{l}\text { Transition } \\
Q(\mathrm{keV}) \\
l(\%) \\
\end{array}$ & $\begin{array}{l}\text { Decay channel, } \\
\text { level of daughter } \\
\text { nuclei }(\mathrm{keV})\end{array}$ & $\Delta(\mathrm{keV})$ & $\begin{array}{c}\text { Expt. } \\
\text { limit (yr) }\end{array}$ & $\begin{array}{l}\text { Experimental } \\
\text { technique } \\
\text { (reference) }\end{array}$ & $\Gamma_{f}(\mathrm{eV})$ & $R_{f}$ \\
\hline & $K L, 2,3^{-} 2748.2(4)$ & $-(0.8-0.3) \pm 0.4$ & $\geq 1.4 \times 10^{21}$ & $\begin{array}{l}\text { Enr. }{ }^{106} \mathrm{CdWO}_{4} \\
\quad \text { scintillator }{ }^{\mathrm{c}}(\text { Belli } \\
\quad \text { et al., 2020) }\end{array}$ & $8.3\left(K L_{3}\right)$ & $1.6 \times 10^{7}$ \\
\hline $\begin{array}{l}{ }^{108} \mathrm{Cd} \rightarrow{ }^{108} \mathrm{Pd} \\
271.8(8) \\
0.888(11)\end{array}$ & $2 \mathrm{EC}, 0^{+}$g.s. & 223.1(8) & $\geq 1.0 \times 10^{18}$ & $\begin{array}{l}\mathrm{CdWO}_{4} \text { scintillator } \\
\quad(\text { Belli et al., 2008b) }\end{array}$ & $12.5(K K)$ & 55 \\
\hline \multirow{4}{*}{$\begin{array}{l}{ }^{112} \mathrm{Sn} \rightarrow{ }^{112} \mathrm{Cd} \\
1919.80(16) \\
0.97(1)\end{array}$} & $K K, 0^{+} 1870.96(5)$ & $-4.62 \pm 0.17$ & $\geq 1.3 \times 10^{21}$ & $\begin{array}{l}\text { HPGe } \gamma \text { spectrometry } \\
\text { of enriched }\end{array}$ & $14.6(K K)$ & $1.5 \times 10^{5}$ \\
\hline & $K K, 0^{+}$g.s. & & $\geq 1.1 \times 10^{21}$ & $\begin{array}{l}{ }_{212}^{11} \text { Sn (Barabash et al., } \\
\text { 2011) }\end{array}$ & & \\
\hline & $K L, 0^{+}$g.s. & & $\geq 8.2 \times 10^{20}$ & & & \\
\hline & $L L, 0^{+}$g.s. & & $\geq 6.4 \times 10^{20}$ & & & \\
\hline \multirow[t]{4}{*}{$\begin{array}{l}{ }^{120} \mathrm{Te} \rightarrow{ }^{120} \mathrm{Sn} \\
1730(3) \\
0.09(1)\end{array}$} & $K K, 0^{+}$g.s. & & $\geq 6.0 \times 10^{17}$ & $\begin{array}{l}\text { HPGe } \gamma \text { spectrometry } \\
\text { (Barabash, Hubert, } \\
\text { Hubert, and Umatov, } \\
\text { 2007) }\end{array}$ & & \\
\hline & $K L, 0^{+}$g.s. & & $\geq 3.9 \times 10^{17}$ & $\begin{array}{l}\text { HPGe } \gamma \text { spectrometry } \\
\text { (Barabash, Hubert, } \\
\text { Hubert, and Umatov, } \\
\text { 2007) }\end{array}$ & & \\
\hline & $L L, 0^{+}$g.s. & & $\geq 2.9 \times 10^{17}$ & $\begin{array}{l}\text { HPGe } \gamma \text { spectrometry } \\
\text { (Barabash, Hubert, } \\
\text { Hubert, and Umatov, } \\
\text { 2007) }\end{array}$ & & \\
\hline & $K K, 2^{+} 1171.265(15)$ & $500 \pm 3$ & & & $17.1(K K)$ & 15 \\
\hline${ }^{124} \mathrm{Xe} \rightarrow{ }^{124} \mathrm{Te}$ & $K K, 0^{+} / 4^{+} 2790.41(9)$ & $10.3 \pm 2.2$ & & & $19.8(K K)$ & $4.1 \times 10^{4}$ \\
\hline $2863.9(22)$ & $K K, 2^{+} 2808.66(8)$ & $-8.0 \pm 2.2$ & & & $19.8(K K)$ & $6.8 \times 10^{4}$ \\
\hline $0.095(5)$ & $\begin{array}{c}K L_{1}, 2^{+} 2817.48(11) \\
L_{1} L_{1}, 2853.2(6)\end{array}$ & $\begin{array}{c}10.1 \pm 2.2 \\
1.2 \pm 2.3\end{array}$ & & & $\begin{array}{l}12.1\left(K L_{1}\right) \\
4.4\left(L_{1} L_{1}\right)\end{array}$ & $\begin{array}{l}2.6 \times 10^{4} \\
6.4 \times 10^{5}\end{array}$ \\
\hline \multirow{2}{*}{$\begin{array}{l}{ }^{126} \mathrm{Xe} \rightarrow{ }^{126} \mathrm{Te} \\
918(4) \\
0.089(3)\end{array}$} & $2 \mathrm{EC}, 0^{+}$g.s. & $854(4)$ & & & $19.8(K K)$ & 6 \\
\hline & $K K, 2^{+} 666.352(10)$ & $188(4)$ & & & $19.8(K K)$ & 122 \\
\hline \multirow{6}{*}{$\begin{array}{l}{ }^{130} \mathrm{Ba} \rightarrow{ }^{130} \mathrm{Xe} \\
2618.9(26) \\
0.11(1)\end{array}$} & $2 \mathrm{EC}, 0^{+}$g.s. & & $\geq 2.8 \times 10^{21}$ & $\begin{array}{l}\text { Geochemical } \\
\quad(\text { Meshik } \text { et al., 2001) }\end{array}$ & & \\
\hline & & & $\geq 7.4 \times 10^{20}$ & $\begin{array}{l}\text { Geochemical } \\
\quad \text { (Pujol et al., 2009) }^{\mathrm{d}}\end{array}$ & & \\
\hline & $K K, 0^{+} 2017.06(16)$ & $532.7 \pm 2.6$ & & & $23.0(K K)$ & 18 \\
\hline & $K K, 1,22502.207(25)$ & $47.6 \pm 2.6$ & & & $23.0(K K)$ & $2.2 \times 10^{3}$ \\
\hline & $K K, 2544.43(8)$ & $5.3 \pm 2.6$ & & & $23.0(K K)$ & $1.5 \times 10^{5}$ \\
\hline & $L_{1} L_{2}, 2608.426(19)$ & $-0.1 \pm 2.6$ & & & $5.1\left(L_{1} L_{2}\right)$ & $1.6 \times 10^{8}$ \\
\hline \multirow{2}{*}{$\begin{array}{l}{ }^{132} \mathrm{Ba} \rightarrow{ }^{132} \mathrm{Xe} \\
843.9(11) \\
0.10(1)\end{array}$} & $2 \mathrm{EC}, 0^{+}$g.s. & $774.8(11)$ & $\geq 2.5 \times 10^{21}$ & $\begin{array}{l}\text { Geochemical } \\
\quad(\text { Meshik et al., 2001) }\end{array}$ & $23.0(K K)$ & 8 \\
\hline & $K K, 2^{+} 667.715(2)$ & $107.1 \pm 1.1$ & & & $23.0(K K)$ & 436 \\
\hline
\end{tabular}


TABLE V. (Continued)

\begin{tabular}{|c|c|c|c|c|c|c|}
\hline $\begin{array}{l}\text { Transition } \\
Q(\mathrm{keV}) \\
\imath(\%) \\
\end{array}$ & $\begin{array}{l}\text { Decay channel, } \\
\text { level of daughter } \\
\text { nuclei }(\mathrm{keV})\end{array}$ & $\Delta(\mathrm{keV})$ & $\begin{array}{c}\text { Expt. } \\
\text { limit (yr) }\end{array}$ & $\begin{array}{c}\text { Experimental } \\
\text { technique } \\
\text { (reference) }\end{array}$ & $\Gamma_{f}(\mathrm{eV})$ & $R_{f}$ \\
\hline \multirow{5}{*}{$\begin{array}{l}{ }^{136} \mathrm{Ce} \rightarrow{ }^{136} \mathrm{Ba} \\
2378.55(27) \\
0.186(2)\end{array}$} & $K K, 0^{+}$g.s. & & $\geq 2.1 \times 10^{18}$ & $\begin{array}{l}\text { HPGe } \gamma \text { spectrometry } \\
\quad(\text { Belli et al., 2017) }\end{array}$ & & \\
\hline & $K L, 0^{+}$g.s. & & $\geq 3.4 \times 10^{18}$ & $\begin{array}{l}\text { HPGe } \gamma \text { spectrometry } \\
\quad \text { (Belli et al., 2017) }\end{array}$ & & \\
\hline & $L L, 0^{+}$g.s. & & $\geq 8.4 \times 10^{18}$ & $\begin{array}{l}\text { HPGe } \gamma \text { spectrometry } \\
\quad \text { (Belli et al., 2017) }\end{array}$ & & \\
\hline & $K K, 0^{+} 2141.38(3)$ & $162.29 \pm 0.27$ & $\geq 4.2 \times 10^{18}$ & $\begin{array}{l}\text { HPGe } \gamma \text { spectrometry } \\
\quad \text { (Belli et al., 2017) }\end{array}$ & $26.4(K K)$ & 218 \\
\hline & $2 \mathrm{EC}, 0^{+} 2315.26(7)$ & $-11.59 \pm 0.28$ & $\geq 2.5 \times 10^{18}$ & $\begin{array}{l}\text { HPGe } \gamma \text { spectrometry } \\
\quad \text { (Belli et al., 2017) }\end{array}$ & $26.4(K K)$ & $4.3 \times 10^{4}$ \\
\hline \multirow{3}{*}{$\begin{array}{l}{ }^{138} \mathrm{Ce} \rightarrow{ }^{138} \mathrm{Ba} \\
691(5) \\
0.251(2)\end{array}$} & $K K, 0^{+}$g.s. & $616(5)$ & $\geq 5.5 \times 10^{17}$ & $\begin{array}{l}\text { HPGe } \gamma \text { spectrometry } \\
\quad(\text { Belli et al., 2014) }\end{array}$ & $26.4(K K)$ & 15 \\
\hline & $K L, 0^{+}$g.s. & & $\geq 8.3 \times 10^{17}$ & $\begin{array}{l}\text { HPGe } \gamma \text { spectrometry } \\
\quad(\text { Belli et al., 2017) }\end{array}$ & & \\
\hline & $L L, 0^{+}$g.s. & & $\geq 4.2 \times 10^{18}$ & $\begin{array}{l}\text { HPGe } \gamma \text { spectrometry } \\
\quad \text { (Belli et al., 2017) }\end{array}$ & & \\
\hline \multirow{5}{*}{$\begin{array}{l}{ }^{144} \mathrm{Sm} \rightarrow{ }^{144} \mathrm{Nd} \\
1782.4(8) \\
3.08(4)\end{array}$} & $K K, 0^{+}$g.s. & & $\geq 4.4 \times 10^{19}$ & $\begin{array}{l}\text { HPGe } \gamma \text { spectrometry } \\
\quad(\text { Belli et al., 2019a) }\end{array}$ & & \\
\hline & $K L, 0^{+}$g.s. & & $\geq 1.7 \times 10^{19}$ & $\begin{array}{l}\text { HPGe } \gamma \text { spectrometry } \\
\quad(\text { Belli et al., 2019a) }\end{array}$ & & \\
\hline & $L L, 0^{+}$g.s. & & $\geq 1.4 \times 10^{19}$ & $\begin{array}{l}\text { HPGe } \gamma \text { spectrometry } \\
\quad(\text { Belli et al., 2019a) }\end{array}$ & & \\
\hline & $K K, 4^{+} 1314.669(13)$ & $380.6 \pm 0.8$ & & & $34.8(K K)$ & 52 \\
\hline & $K K, 2^{+} 1560.920(13)$ & $134.3 \pm 0.8$ & & & $34.8(K K)$ & 419 \\
\hline $\begin{array}{l}{ }^{152} \mathrm{Gd} \rightarrow{ }^{152} \mathrm{Sm} \\
55.69(18) \\
0.20(3)\end{array}$ & $\begin{array}{l}\text { All transitions } \\
K L, 0^{+} \text {g.s. }\end{array}$ & $(1.1-2.1) \pm 0.2$ & $\begin{array}{l}\geq 6.0 \times 10^{8} \\
\geq 6.0 \times 10^{8}\end{array}$ & $\begin{array}{l}\text { Analysis of average } \\
\text { parent-daughter } \\
\text { abundances } \\
\text { (Nozzoli, 2018) }\end{array}$ & $23.1\left(K L_{1}\right)$ & $4.1 \times 10^{6}$ \\
\hline \multirow{7}{*}{$\begin{array}{l}{ }^{156} \mathrm{Dy} \rightarrow{ }^{156} \mathrm{Gd} \\
2005.95(10) \\
0.056(3)\end{array}$} & $K K, 0^{+}$g.s. & & $\geq 2.2 \times 10^{16}$ & $\begin{array}{l}\text { HPGe } \gamma \text { spectrometry } \\
\quad(\text { Belli et al., 2011a) }\end{array}$ & & \\
\hline & $K L, 0^{+}$g.s. & & $\geq 1.7 \times 10^{16}$ & $\begin{array}{l}\text { HPGe } \gamma \text { spectrometry } \\
\quad \text { (Belli et al., 2011a) }\end{array}$ & & \\
\hline & $L L, 0^{+}$g.s. & & $\geq 1.7 \times 10^{16}$ & $\begin{array}{l}\text { HPGe } \gamma \text { spectrometry } \\
\quad \text { (Belli et al., 2011a) }\end{array}$ & & \\
\hline & $K K, 2^{+} 1914.835(5)$ & $-9.33 \pm 0.10$ & $\geq 1.1 \times 10^{16}$ & $\begin{array}{l}\text { HPGe } \gamma \text { spectrometry } \\
\quad(\text { Belli et al., 2011a) }\end{array}$ & $44.8(K K)$ & $1.1 \times 10^{5}$ \\
\hline & $K L_{1}, 1^{-} 1946.344(10)$ & $0.99 \pm 0.10$ & $\geq 1.0 \times 10^{18}$ & $\begin{array}{l}\text { HPGe } \gamma \text { sp. of enr. } \\
{ }^{156} \text { Dy (Finch and } \\
\text { Tornow, 2015) }\end{array}$ & $26.2\left(K L_{1}\right)$ & $5.8 \times 10^{6}$ \\
\hline & $K L_{1}, 0^{-} 1952.400(6)$ & $-5.06 \pm 0.10$ & $\geq 2.2 \times 10^{17}$ & $\begin{array}{l}\text { HPGe } \gamma \text { sp. of enr. } \\
{ }^{156} \text { Dy (Finch and } \\
\text { Tornow, 2015) }\end{array}$ & $26.2\left(K L_{1}\right)$ & $2.2 \times 10^{5}$ \\
\hline & $L L_{1}, 0^{+} 1988.5(2)$ & $0.7 \pm 0.22$ & $\geq 9.5 \times 10^{17}$ & $\begin{array}{l}\text { HPGe } \gamma \text { sp. of enr. } \\
{ }^{156} \text { Dy (Finch and } \\
\text { Tornow, 2015) }\end{array}$ & $7.6\left(L_{1} L_{1}\right)$ & $3.4 \times 10^{6}$ \\
\hline
\end{tabular}


TABLE V. (Continued)

\begin{tabular}{|c|c|c|c|c|c|c|}
\hline $\begin{array}{l}\text { Transition } \\
Q(\mathrm{keV}) \\
\imath(\%) \\
\end{array}$ & $\begin{array}{l}\text { Decay channel, } \\
\text { level of daughter } \\
\text { nuclei }(\mathrm{keV})\end{array}$ & $\Delta(\mathrm{keV})$ & $\begin{array}{c}\text { Expt. } \\
\text { limit (yr) }\end{array}$ & $\begin{array}{c}\text { Experimental } \\
\text { technique } \\
\text { (reference) }\end{array}$ & $\Gamma_{f}(\mathrm{eV})$ & $R_{f}$ \\
\hline & $L L_{3}, 2^{+} 2003.749(5)$ & $-12.28 \pm 0.10$ & $\geq 6.7 \times 10^{16}$ & $\begin{array}{l}\text { HPGe } \gamma \text { sp. of enr. } \\
\text { 156 Dy (Finch and } \\
\text { Tornow, 2015) }\end{array}$ & $7.4\left(L_{3} L_{3}\right)$ & $1.1 \times 10^{4}$ \\
\hline \multirow{3}{*}{$\begin{array}{l}{ }^{158} \mathrm{Dy} \rightarrow{ }^{158} \mathrm{Gd} \\
282.2(24) \\
0.095(3)\end{array}$} & $K K, 0^{+}$g.s. & $181.7(24)$ & $\geq 4.2 \times 10^{16}$ & $\begin{array}{l}\text { HPGe } \gamma \text { spectrometry } \\
\quad(\text { Belli et al., 2011a) }\end{array}$ & $44.8(K K)$ & 295 \\
\hline & $K K, 2^{+} 79.5143(15)$ & $102.2 \pm 2.4$ & $\geq 2.6 \times 10^{14}$ & $\begin{array}{l}\text { HPGe } \gamma \text { spectrometry } \\
\quad(\text { Belli et al., 2011a) }\end{array}$ & $44.8(K K)$ & 932 \\
\hline & $L_{1} L_{1}, 4^{+} 261.4580(16)$ & $4.0 \pm 2.4$ & $\geq 3.2 \times 10^{16}$ & $\begin{array}{l}\text { HPGe } \gamma \text { spectrometry } \\
\quad(\text { Belli et al., 2011a) }\end{array}$ & $7.6\left(L_{1} L_{1}\right)$ & $1.0 \times 10^{5}$ \\
\hline \multirow{6}{*}{$\begin{array}{l}{ }^{162} \mathrm{Er} \rightarrow{ }^{162} \mathrm{Dy} \\
1846.96(30) \\
0.139(5)\end{array}$} & $K K, 0^{+}$g.s. & & $\geq 1.0 \times 10^{18}$ & $\begin{array}{r}\text { HPGe } \gamma \text { spectrometry } \\
\text { (Belli et al., 2018) }\end{array}$ & & \\
\hline & $K L, 0^{+}$g.s. & & $\geq 9.6 \times 10^{17}$ & $\begin{array}{r}\text { HPGe } \gamma \text { spectrometry } \\
\text { (Belli et al., 2018) }\end{array}$ & & \\
\hline & $L L, 0^{+}$g.s. & & $\geq 1.3 \times 10^{18}$ & $\begin{array}{r}\text { HPGe } \gamma \text { spectrometry } \\
\text { (Belli et al., 2018) }\end{array}$ & & \\
\hline & $K K, 3^{-} 1738.999(4)$ & $0.4 \pm 0.3$ & $\geq 5.5 \times 10^{8}$ & $\begin{array}{l}\text { Analysis of average } \\
\text { parent-daughter } \\
\text { abundances } \\
\text { (Nozzoli, 2018) }\end{array}$ & $50.6(K K)$ & $7.4 \times 10^{7}$ \\
\hline & $K L, 2^{+} 1782.68(9)$ & $(1.4-2.7) \pm 0.3$ & $\geq 5.0 \times 10^{17}$ & $\begin{array}{r}\text { HPGe } \gamma \text { spectrometry } \\
\text { (Belli et al., 2018) }\end{array}$ & $29.6\left(K L_{1}\right)$ & $3.1 \times 10^{6}$ \\
\hline & $L L, 4^{-} 1826.753(4)$ & $(2.1-4.6) \pm 0.3$ & $\geq 5.5 \times 10^{8}$ & $\begin{array}{l}\text { Analysis of average } \\
\text { parent-daughter } \\
\text { abundances } \\
\text { (Nozzoli, 2018) }\end{array}$ & $8.6\left(L_{1} L_{1}\right)$ & $4.2 \times 10^{5}$ \\
\hline \multirow[t]{2}{*}{$\begin{array}{c}{ }^{164} \mathrm{Er} \rightarrow{ }^{164} \mathrm{Dy} \\
25.08(11) \\
1.601(3)\end{array}$} & All transitions & & $\geq 1.0 \times 10^{9}$ & $\begin{array}{l}\text { Analysis of average } \\
\text { parent-daughter } \\
\text { abundances }\end{array}$ & & \\
\hline & $L_{1} L_{1}, 0^{+}$g.s. & $6.99 \pm 0.11$ & & $\left(\right.$ Nozzoli, 2018) ${ }^{\mathrm{f}}$ & $8.6\left(L_{1} L_{1}\right)$ & $3.8 \times 10^{4}$ \\
\hline \multirow{5}{*}{$\begin{array}{c}{ }^{168} \mathrm{Yb} \rightarrow{ }^{168} \mathrm{Er} \\
1409.27(25) \\
0.123(3)\end{array}$} & $K K, 0^{+}$g.s. & & $\geq 7.3 \times 10^{17}$ & $\begin{array}{l}\text { HPGe } \gamma \text { spectrometry } \\
\quad(\text { Belli et al., 2019b) }\end{array}$ & & \\
\hline & $K L, 0^{+}$g.s. & & $\geq 6.9 \times 10^{17}$ & $\begin{array}{l}\text { HPGe } \gamma \text { spectrometry } \\
\quad(\text { Belli et al., 2019b) }\end{array}$ & & \\
\hline & $L L, 0^{+}$g.s. & & $\geq 1.2 \times 10^{18}$ & $\begin{array}{l}\text { HPGe } \gamma \text { spectrometry } \\
\quad(\text { Belli et al., 2019b) }\end{array}$ & & \\
\hline & $2 \mathrm{EC}, 0^{+} 1217.169(14)$ & $77.13 \pm 0.25$ & $\geq 1.5 \times 10^{18}$ & $\begin{array}{l}\text { HPGe } \gamma \text { spectrometry } \\
\quad(\text { Belli et al., 2019b) }\end{array}$ & $57.0(K K)$ & $2.1 \times 10^{3}$ \\
\hline & $\begin{array}{l}M_{1} M_{1}(2)^{-} \\
1403.7357(23)\end{array}$ & $1.12 \pm 0.25$ & $\geq 1.9 \times 10^{18}$ & $\begin{array}{l}\text { HPGe } \gamma \text { spectrometry } \\
\quad(\text { Belli et al., 2019b) }\end{array}$ & $27.2\left(M_{1} M_{1}\right)$ & $4.7 \times 10^{6}$ \\
\hline \multirow{4}{*}{$\begin{array}{c}{ }^{174} \mathrm{Hf} \rightarrow{ }^{174} \mathrm{Yb} \\
1100.0(23) \\
0.16(12)\end{array}$} & $K K, 0^{+}$g.s. & & $\geq 5.8 \times 10^{17}$ & $\begin{array}{l}\text { HPGe } \gamma \text { spectrometry } \\
\quad \text { (Danevich } \text { et al., 2020) }\end{array}$ & $64.0(K K)$ & 15 \\
\hline & $K L, 0^{+}$g.s. & & $\geq 1.9 \times 10^{18}$ & $\begin{array}{l}\text { HPGe } \gamma \text { spectrometry } \\
\quad(\text { Danevich } \text { et al., 2020) }\end{array}$ & & \\
\hline & $L L, 0^{+}$g.s. & & $\geq 7.8 \times 10^{17}$ & $\begin{array}{l}\text { HPGe } \gamma \text { spectrometry } \\
\quad(\text { Danevich } \text { et al., 2020) }\end{array}$ & & \\
\hline & $K K, 2^{+} 76.471(1)$ & $900.9 \pm 2.3$ & $\geq 7.1 \times 10^{17}$ & $\begin{array}{l}\text { HPGe } \gamma \text { spectrometry } \\
\text { (Danevich et al., 2020) }\end{array}$ & $64.0(K K)$ & 17 \\
\hline
\end{tabular}


TABLE V. (Continued)

\begin{tabular}{|c|c|c|c|c|c|c|}
\hline $\begin{array}{l}\text { Transition } \\
Q(\mathrm{keV}) \\
\iota(\%) \\
\end{array}$ & $\begin{array}{l}\text { Decay channel, } \\
\text { level of daughter } \\
\text { nuclei }(\mathrm{keV})\end{array}$ & $\Delta(\mathrm{keV})$ & $\begin{array}{c}\text { Expt. } \\
\text { limit (yr) }\end{array}$ & $\begin{array}{l}\text { Experimental } \\
\text { technique } \\
\text { (reference) }\end{array}$ & $\Gamma_{f}(\mathrm{eV})$ & $R_{f}$ \\
\hline & $K L, 2^{+} 76.471(1)$ & & $\geq 6.2 \times 10^{17}$ & $\begin{array}{l}\text { HPGe } \gamma \text { spectrometry } \\
\quad(\text { Danevich } \text { et al. }, 2020)\end{array}$ & & \\
\hline & $L L, 2^{+} 76.471(1)$ & & $\geq 7.2 \times 10^{17}$ & $\begin{array}{l}\text { HPGe } \gamma \text { spectrometry } \\
\quad(\text { Danevich } \text { et al., 2020) }\end{array}$ & & \\
\hline \multirow{3}{*}{$\begin{array}{l}{ }^{180} \mathrm{~W} \rightarrow{ }^{180} \mathrm{Hf} \\
143.23(28) \\
0.12(1)\end{array}$} & $K K, 0^{+}$g.s. & $12.53 \pm 0.28$ & $\geq 9.4 \times 10^{18}$ & $\begin{array}{r}\mathrm{CaWO}_{4} \text { scint. bolometer } \\
\text { (Angloher } \text { et al., 2016) }\end{array}$ & $71.8(K K)$ & $9.9 \times 10^{4}$ \\
\hline & & & $\geq 1.3 \times 10^{18}$ & $\begin{array}{l}\mathrm{ZnWO}_{4} \text { scintillator } \\
\quad \text { (Belli, Bernabei et al., } \\
\text { 2011b) }\end{array}$ & & \\
\hline & $L_{1} L_{1}, 2^{+} 93.3240(20)$ & $27.36 \pm 0.28$ & & & $11.4\left(L_{1} L_{1}\right)$ & $3.3 \times 10^{3}$ \\
\hline \multirow{7}{*}{$\begin{array}{l}{ }^{184} \mathrm{Os} \rightarrow{ }^{184} \mathrm{~W} \\
1452.8(7) \\
0.02(2)\end{array}$} & $K K, 0^{+}$g.s. & & $\geq 2.0 \times 10^{17}$ & $\begin{array}{l}\text { HPGe } \gamma \text { spectrometry } \\
\quad \text { (Belli et al., 2013a) }\end{array}$ & & \\
\hline & $K L, 0^{+}$g.s. & & $\geq 1.3 \times 10^{17}$ & $\begin{array}{l}\text { HPGe } \gamma \text { spectrometry } \\
\quad(\text { Belli et al., 2013a) }\end{array}$ & & \\
\hline & $L L, 0^{+}$g.s. & & $\geq 1.4 \times 10^{17}$ & $\begin{array}{l}\text { HPGe } \gamma \text { spectrometry } \\
\quad(\text { Belli et al., 2013a) }\end{array}$ & & \\
\hline & $2 \mathrm{EC}, 0^{+} 1002.49(4)$ & $311.3 \pm 0.7$ & $\geq 3.5 \times 10^{17}$ & $\begin{array}{l}\text { HPGe } \gamma \text { spectrometry } \\
\text { (Belli et al., 2013a) }\end{array}$ & $80.2(K K)$ & 180 \\
\hline & $K K,(0)^{+} 1322.152(22)$ & $-8.4 \pm 0.7$ & $\geq 2.8 \times 10^{16}$ & $\begin{array}{l}\text { HPGe } \gamma \text { spectrometry } \\
\quad(\text { Belli et al., 2013a) }\end{array}$ & $80.2(K K)$ & $2.5 \times 10^{5}$ \\
\hline & $K L, 2^{+} 1386.296(13)$ & $-(13.2-15.1) \pm 0.7$ & $\geq 6.7 \times 10^{16}$ & $\begin{array}{l}\text { HPGe } \gamma \text { spectrometry } \\
\quad \text { (Belli et al., 2013a) }\end{array}$ & $46.4\left(K L_{1}\right)$ & $4.4 \times 10^{4}$ \\
\hline & $L L, 2^{+} 1431.02(5)$ & $(-2.4-+1.4) \pm 0.7$ & $\geq 8.2 \times 10^{16}$ & $\begin{array}{l}\text { HPGe } \gamma \text { spectrometry } \\
\quad \text { (Belli et al., 2013a) }\end{array}$ & $9.6\left(L_{2} L_{3}\right)$ & $2.4 \times 10^{9}$ \\
\hline \multirow{6}{*}{$\begin{array}{l}{ }^{190} \mathrm{Pt} \rightarrow{ }^{190} \mathrm{Os} \\
1401.3(4) \\
0.012(2)\end{array}$} & $K K, 0^{+}$g.s. & & $\geq 5.7 \times 10^{15}$ & $\begin{array}{l}\text { HPGe } \gamma \text { spectrometry } \\
\text { (Belli et al., 2011b) }\end{array}$ & & \\
\hline & $K L, 0^{+}$g.s. & & $\geq 1.7 \times 10^{16}$ & $\begin{array}{l}\text { HPGe } \gamma \text { spectrometry } \\
\quad(\text { Belli et al., 2011b) }\end{array}$ & & \\
\hline & $L L, 0^{+}$g.s. & & $\geq 3.1 \times 10^{16}$ & $\begin{array}{l}\text { HPGe } \gamma \text { spectrometry } \\
\quad(\text { Belli et al., 2011b) }\end{array}$ & & \\
\hline & $K N, 1,21326.9(5)$ & $(-0.1-+0.3) \pm 0.6$ & & & $52.3\left(K N_{1}\right)$ & $7.0 \times 10^{8}$ \\
\hline & $L M,(0,1,2)^{+} 1382.4(2)$ & $(2.9-6.1) \pm 0.5$ & $\geq 2.9 \times 10^{16}$ & $\begin{array}{l}\text { HPGe } \gamma \text { spectrometry } \\
\quad(\text { Belli et al., 2011b) }\end{array}$ & $21.9\left(L_{1} M_{1}\right)$ & $5.7 \times 10^{5}$ \\
\hline & $L_{3} M_{3}, 3^{-} 1387.00(2)$ & $1.0 \pm 0.4$ & & & $12.6\left(L_{3} M_{3}\right)$ & $2.9 \times 10^{6}$ \\
\hline $\begin{array}{l}{ }^{196} \mathrm{Hg} \rightarrow{ }^{196} \mathrm{Pt} \\
818.6(30)\end{array}$ & $K K, 0^{+}$g.s. & $661.8 \pm 3.0$ & $\geq 2.5 \times 10^{18}$ & $\begin{array}{l}\mathrm{Ge}(\mathrm{Li}) \gamma \text { spectrometry } \\
\quad(\text { Bukhner } \text { et al. }, 1990)\end{array}$ & $99(K K)$ & 49 \\
\hline $0.15(1)$ & $K L, 2^{+} 688.693(5)$ & $37.6 \pm 3.0$ & & & $58.3\left(K L_{1}\right)$ & $8.9 \times 10^{3}$ \\
\hline
\end{tabular}

${ }^{a}$ The result $4.3 \times 10^{19} \mathrm{yr}$ of Frekers et al. (2011) for ${ }^{74}$ Se was corrected to $1.4 \times 10^{18}$ yr by Ješkovský et al. (2015) and reestimated as $3.9 \times 10^{16} \mathrm{yr}$ by Lehnert, Wester et al. (2016). See those works for details.

${ }^{\mathrm{b}}$ The result $1.5 \times 10^{19} \mathrm{yr}$ of Ješkovský et al. (2015) for ${ }^{74} \mathrm{Se}$ was reestimated as $6.3 \times 10^{17}$ yr by Lehnert, Wester et al. (2016).

${ }^{c}$ In coincidence with $\mathrm{CdWO}_{4}$ counters.

${ }^{\mathrm{d}}$ The limit is valid for all possible $2 \beta$-decay modes in ${ }^{130} \mathrm{Ba}$.

${ }^{\mathrm{e}}$ The limit is valid for all possible 2EC-decay modes in ${ }^{132} \mathrm{Ba}$.

${ }^{\mathrm{f}}$ The limit is valid for all possible 2EC-decay modes in ${ }^{164} \mathrm{Er}$. 


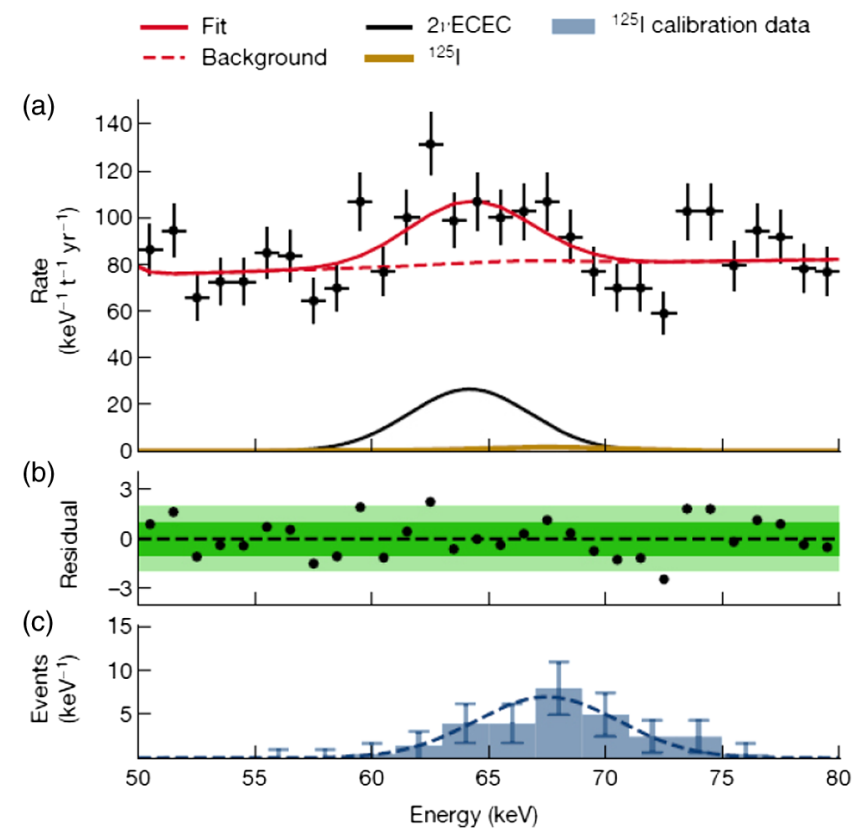

FIG. 10. Result of the XENON1t data analysis in the region of interest for $2 \nu 2 \mathrm{EC}$ in ${ }^{124} \mathrm{Xe}$. (a) $2 \nu 2 \mathrm{EC}$ decay peak with area 126 events, obtained using the fit of the experimental data. (b) Residuals for the experimental data best fit. (c) Histogram of the ${ }^{125} \mathrm{I}$ peak produced by neutron activation after the detector calibrations with an external ${ }^{241} \mathrm{AmBe}$ neutron source. The peak area in (a) corresponds to a half-life of ${ }^{124} \mathrm{Xe}$ of $1.8 \times 10^{22} \mathrm{yr}$. From XENON Collaboration, 2019.

of the $2 \nu 2 \mathrm{EC}$ in ${ }^{78} \mathrm{Kr}$ with the half-life of $T_{1 / 2}=\left[9.2_{-2.6}^{+5.5}(\right.$ stat $) \pm$ 1.3 (syst) $] \times 10^{21}$ yr was obtained by Gavrilyuk et al. (2013). The indications of barium 2EC decay should be confirmed in direct counting experiments, while the ${ }^{78} \mathrm{Kr}$ and ${ }^{124} \mathrm{Xe}$ results also need to be confirmed with higher statistics in independent experiments.

In Table $\mathrm{V}$, the experimental limits on neutrinoless 2EC half-lives are presented. The data are given for the transitions for which the probability is expected to be greatest: either to the ground state or to the excited levels of the daughter nuclide with possible resonant enhancement of the decay rate. The deexcitation width of the electron shell of the daughter nuclides $\Gamma_{f}=\Gamma_{\alpha}+\Gamma_{\beta}$ and the resonance parameter $R_{f}=\Gamma_{f} /\left(\Delta^{2}+\Gamma_{f}^{2} / 4\right)$ (normalized on the $R_{f}$ value for ${ }^{54} \mathrm{Fe}$ ) are also included in the table to indicate the status of the transitions as potential resonances.

The experiments to investigate double-beta-plus processes can be divided into three groups depending on the experimental technique: geochemical investigations [the only examples are the searches for $2 \beta$ decay of barium (Meshik et al., 2001; Pujol et al., 2009; Meshik and Pravdivtseva, 2017)], $\gamma$ spectrometry (the search for $\gamma$ quanta expected in the decay processes when using $\gamma$ spectrometers, mainly HPGe detectors), and the calorimetric approach ( $2 \beta$ active nuclei are incorporated into a detector). For instance, investigations of ${ }^{106} \mathrm{Cd}$ were realized by using the last two methods: the search for $\gamma$ quanta from enriched ${ }^{106} \mathrm{Cd}$ samples with HPGe (Barabash et al., 1996; Rukhadze et al., 2011a, 2011b) or $\mathrm{NaI}(\mathrm{Tl})$ detectors (Belli, Bernabei et al., 1999)
( $\gamma$ spectrometry), and with the help of a CdTe cryogenic bolometer (Ito et al., 1997), CdZnTe diodes (Dawson et al., 2009b), and a cadmium tungstate crystal scintillator enriched in ${ }^{106} \mathrm{Cd}\left({ }^{106} \mathrm{CdWO}_{4}\right)$ (Belli et al., 2012b) (calorimetric approach). Figure 11 presents a $\gamma$ spectrometry approach used in the TGV-2 experiment.

The proportional chamber filled with enriched ${ }^{78} \mathrm{Kr}$ used in the experiment by Gavrilyuk et al. (2013) is an example of the calorimetric approach; see Fig. 12, left panel. CdZnTe diodes were applied by the COBRA Collaboration to investigate double-beta processes in $\mathrm{Zn}, \mathrm{Cd}$, and Te isotopes; see Fig. 12, right panel. Another case are scintillation calorimetric experiments, as, for instance, the experiment to search for $2 \beta$ decay of ${ }^{106} \mathrm{Cd}$ with the help of the ${ }^{106} \mathrm{CdWO}_{4}$ crystal scintillator (Belli et al., 2012b). The next stages of the experiment with the ${ }^{106} \mathrm{CdWO}_{4}$ scintillation detector operated in coincidence with four HPGe $\gamma$ detectors (Belli et al., 2016) and with two $\mathrm{CdWO}_{4}$ counters (Belli et al., 2020) represent a combination of the last two approaches.

An advantage of the $\gamma$ spectrometry method is the high-energy resolution of HPGe detectors (approximately a few $\mathrm{keV}$ ). However, the typical detection efficiency of HPGe $\gamma$ detectors is rather low: on the level of fractions of a percent to several percent depending on the decay mode and the setup configuration. ${ }^{4}$ The detection efficiency of the calorimetric method is much higher. Depending on the decay mode, energy threshold, detector volume, and composition, the detection efficiency can approach $100 \% .^{5}$ In addition, the calorimetric method allows one to distinguish between different modes and channels of $2 \beta$ processes. Examples of Monte Carlo simulated responses of the ${ }^{106} \mathrm{CdWO}_{4}$ detector to different channels and modes of $2 \beta$ decay of ${ }^{106} \mathrm{Cd}$ are presented in Fig. 13.

However, the $\gamma$ spectrometry approach can clearly distinguish between the $2 \nu$ and $0 \nu$ modes of the $2 \mathrm{EC}$ decay too. The $2 \nu 2 \mathrm{EC}$ decay to the ground state of the daughter nucleus gives a cascade of $x$ rays and Auger electrons, with the individual energies scaling up to the maximal energy of the $K$ x rays. A combination of $\mathrm{X}$-ray peaks is expected in the decay [as shown in Fig. 14 (top panel) for ${ }^{190} \mathrm{Pt}$ ]; $\mathrm{x}$ rays from other atomic shells $(L, M, \ldots)$ can be emitted in the $2 \nu 2 \mathrm{EC}$ decay too. However, detection of such small energies by HPGe $\gamma$ spectrometry is problematic given that even in the case of one of the heaviest 2EC nuclides ${ }^{190} \mathrm{Pt}$ the energy of $L \times$ rays varies from 9 to $13 \mathrm{keV}$.

In the process of $0 \nu 2 \mathrm{EC}$ decay, in addition to $\mathrm{x}$ rays, it is expected that one or more inner-bremsstrahlung photons are emitted, carrying off the total decay energy, which in the $2 \nu$ process is taken by the neutrinos (Rosen and Primakoff, 1965). The energy of the $\gamma$ quanta is expected to be equal to $E_{\gamma}=Q-\epsilon_{\alpha \beta}^{*}$, where $\epsilon_{\alpha \beta}^{*}$ is the excitation energy of the atomic shell with two vacancies $\alpha$ and $\beta$ of the

${ }^{4}$ See Barabash, Hubert, Hubert, and Umatov (2007), Barabash et al. (2011), Belli et al. (2011a, 2011b, 2013a, 2013b), and Rukhadze et al. (2011a).

${ }^{5}$ See Kiel, Münstermann, and Zuber (2003), Andreotti et al. (2011), Belli, Bernabei et al. (2011b), Belli et al. (2012b), Gavrilyuk et al. (2013), Mei et al. (2014), and Angloher et al. (2016). 

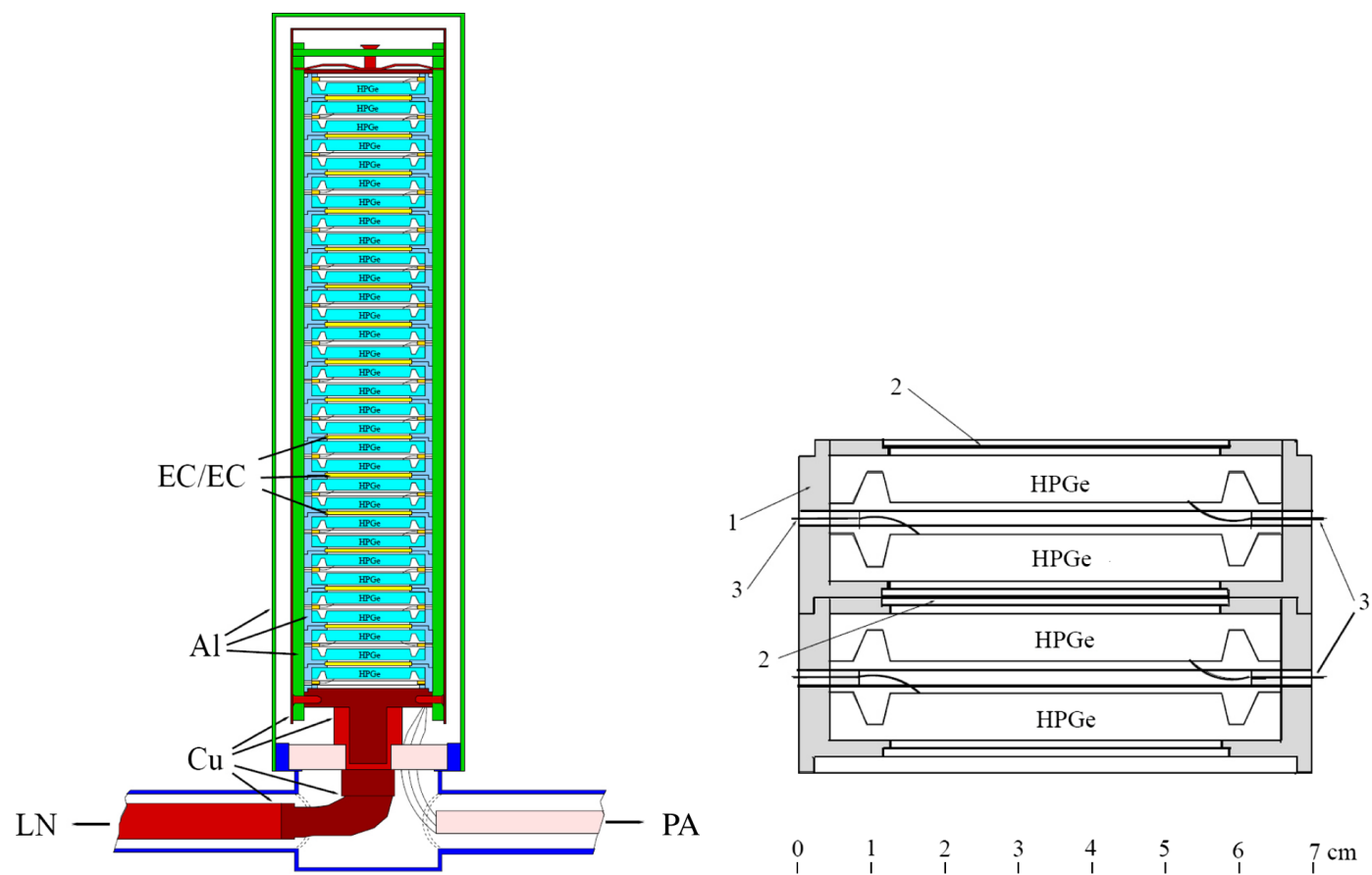

PA

$\begin{array}{llllllll}0 & 1 & 2 & 3 & 4 & 5 & 6 & 7 \\ 1 & 1 & 1 & 1 & 1 & 1 & 1 & 1\end{array}$

FIG. 11. Left panel: schematic view of the TGV-2 detector. HPGe, planar-type Ge detectors; EC/EC, enriched ${ }^{106} \mathrm{Cd}$ foils; $\mathrm{Al}$, construction details made from Al-Si alloy; $\mathrm{Cu}$, construction details made from copper; LN, liquid nitrogen; PA, preamplifiers. Right panel: section view of the stack of HPGe detectors. 1, cylindrical holders for the detectors; $2,{ }^{106} \mathrm{Cd}$ foils; 3 , electric contacts (bronze wires in Teflon insulators). From Rukhadze et al., 2006.
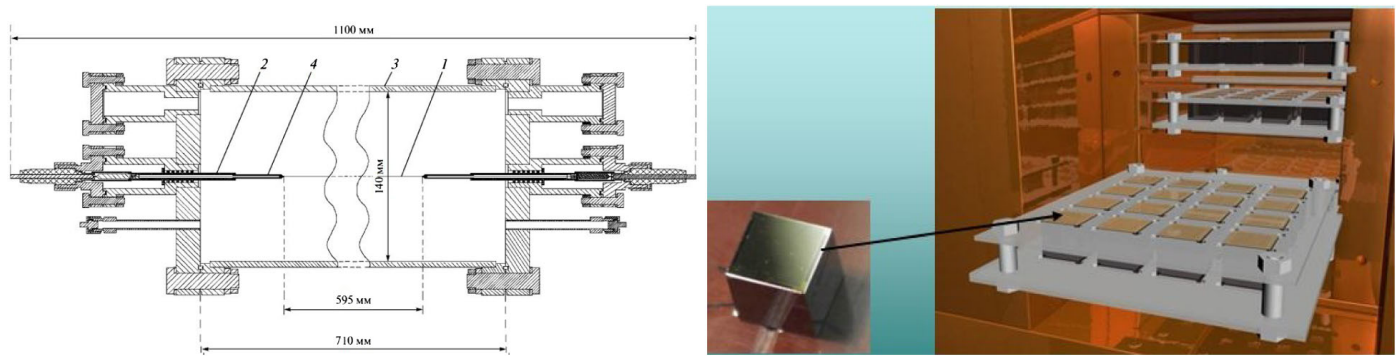

FIG. 12. Left panel: low counting proportional chamber filled with enriched ${ }^{78} \mathrm{Kr}$ used in the experiment by Gavrilyuk et al. (2013). 1, anode wire; 2, insulator; 3, cathode; 4, copper tubes. From Gavrilyuk et al., 2010. Right panel: concept of the COBRA CdZnTe detectors array. From K. Zuber.

daughter nucleus. Therefore, the expected energies of the quanta for the $0 \nu 2 \mathrm{EC}$ decay to the g.s.'s of the daughter nucleus are much higher than those in the $2 \nu 2 \mathrm{EC}$ decay; see Fig. 14 (bottom panel).
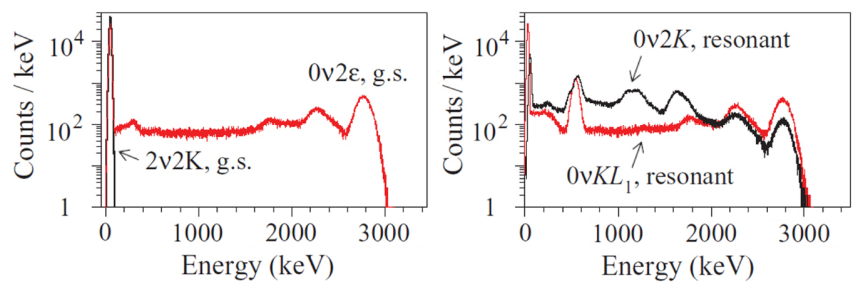

FIG. 13. Monte Carlo simulated energy spectra of $2 \nu K K$, $0 \nu 2 \mathrm{EC}$ decays of ${ }^{106} \mathrm{Cd}$ to the ground state of ${ }^{106} \mathrm{Pd}$, and neutrinoless resonant transitions of ${ }^{106} \mathrm{Cd}$ to excited levels in ${ }^{106}$ Pd. From Belli et al., 2012b.
There are several reasons for the lower sensitivity of the $2 \beta^{+}$experiments (in comparison to the $2 \beta^{-}$ones). First, the development of experimental techniques and the scale of the experiments are rather modest. For example, the amount of enriched isotopes utilized does not exceed tens of grams, while tens or even hundreds of kilograms of isotopically enriched materials have already been used in $2 \beta^{-}$experiments. Then one should take into account the much lower abundance of $2 \beta^{+}$active isotopes $(l)$ in the natural isotopic compositions of elements, which is usually lower than $1 \%$. There are only six nuclei, namely, ${ }^{40} \mathrm{Ca}(l=96.941 \%),{ }^{54} \mathrm{Fe}(5.845 \%),{ }^{58} \mathrm{Ni}$ $(68.0769 \%),{ }^{64} \mathrm{Zn}(49.17 \%),{ }^{92} \mathrm{Mo}(14.649 \%)$, and ${ }^{96} \mathrm{Ru}$ (5.54\%) from the full list of 34 double-beta-plus candidates with $l$ values greater than 5\% (Tretyak and Zdesenko, 2002; Meija et al., 2016). For comparison, from the full list of 35 potentially $2 \beta^{-}$decaying nuclides, only five candidates have $\imath$ below $5 \%$. We note that starting from ${ }^{74} \mathrm{Se}$ the list of 

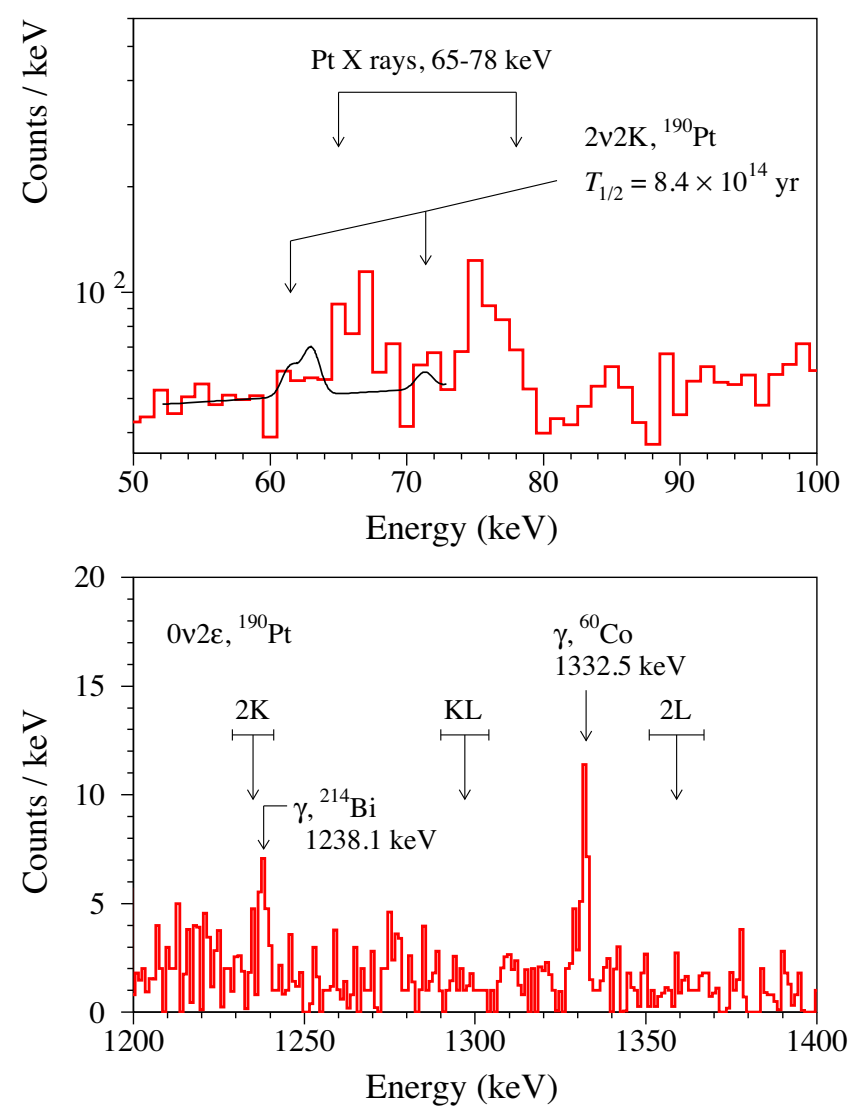

FIG. 14. Upper panel: low-energy part of the spectrum accumulated by a HPGe detector with a platinum sample over $1815 \mathrm{~h}$. The distribution expected for the $2 \nu K K$ decay of ${ }^{190} \mathrm{Pt}$ with a halflife of $8.4 \times 10^{14} \mathrm{yr}$ excluded at $90 \%$ C.L. is shown with a solid line. Lower panel: part of the energy spectrum accumulated with the platinum sample in the energy region where peaks from the $0 \nu 2 \mathrm{EC}$ processes in ${ }^{190} \mathrm{Pt}(K K, K L$, and $L L)$ to the ground state of ${ }^{190} \mathrm{Os}$ are expected. The error bars take into account the uncertainty of the $Q$ value of $2 \mathrm{EC}$ in ${ }^{190} \mathrm{Pt}$ available at the time when the experiment was realized $( \pm 6 \mathrm{keV})$ and different energies of the $L \mathrm{x}$ rays of osmium. From Belli et al., $2011 \mathrm{~b}$.

$2 \beta^{+} / \mathrm{EC} \beta^{+} / 2 \mathrm{EC}$ nuclei practically coincides with the list of the so-called bypassed (or $p$ ) nuclei (Frank-Kamenetsky and Domogatsky, 1986), which cannot be created in usual $r$ and $s$ processes of nucleosynthesis by successive neutron captures, and whose abundances, as a result, are suppressed in comparison to those of $r$ and $s$ nuclei.

Finally, the released energy in the $2 \beta^{+}$and $\mathrm{EC} \beta^{+}$decays is lower than that in the $2 \beta^{-}$decays, which results in a lower probability for such processes due to the small phase-space factors. For $0 \nu 2 \beta^{-}$-decay processes, because of the relation among half-life $T_{1 / 2}^{0 \nu}$, phase-space factor $G_{0 \nu}$, the NME $\left(M^{0 \nu}\right)$, and $m_{\beta \beta}: 1 / T_{1 / 2}^{0 \nu}=G_{0 \nu}\left|M^{0 \nu}\right|^{2}\left|m_{\beta \beta}\right|^{2}$, the lower value of $G_{0 \nu}$ for $2 \beta^{+} / \mathrm{EC} \beta^{+}$processes results in a weaker limit on the effective neutrino mass or other parameters, even if the same experimental $T_{1 / 2}$ limit is reached as for the $2 \beta^{-}$decay. To undergo $2 \beta^{+}$decay, the energy release should exceed four electron masses, and only six nuclei from the full list of 34 have enough energy for this: ${ }^{78} \mathrm{Kr}(Q=2848 \mathrm{keV}),{ }^{96} \mathrm{Ru}(2715 \mathrm{keV}),{ }^{106} \mathrm{Cd}$ $(2775 \mathrm{keV}),{ }^{124} \mathrm{Xe}(2864 \mathrm{keV}),{ }^{130} \mathrm{Ba}(2619 \mathrm{keV})$, and ${ }^{136} \mathrm{Ce}$
(2379 keV). All these nuclides, together with 16 additional nuclides ${ }^{50} \mathrm{Cr},{ }^{58} \mathrm{Ni},{ }^{64} \mathrm{Zn},{ }^{74} \mathrm{Se},{ }^{84} \mathrm{Sr},{ }^{92} \mathrm{Mo},{ }^{102} \mathrm{Pd},{ }^{112} \mathrm{Sn},{ }^{120} \mathrm{Te}$, ${ }^{144} \mathrm{Sm},{ }^{156} \mathrm{Dy},{ }^{162} \mathrm{Er},{ }^{168} \mathrm{Yb},{ }^{174} \mathrm{Hf},{ }^{184} \mathrm{Os}$, and ${ }^{190} \mathrm{Pt}$, undergo $\mathrm{EC} \beta^{+}$decays for which the released energy should exceed $2 m_{e}+\epsilon_{\alpha}^{*}$, where $\epsilon_{\alpha}^{*}$ is the binding energy of the captured electron. And all of these 22 nuclides, together with the 12 remaining ones ${ }^{36} \mathrm{Ar},{ }^{40} \mathrm{Ca},{ }^{54} \mathrm{Fe},{ }^{108} \mathrm{Cd},{ }^{126} \mathrm{Xe},{ }^{132} \mathrm{Ba},{ }^{138} \mathrm{Ce}$, ${ }^{152} \mathrm{Gd},{ }^{158} \mathrm{Dy},{ }^{164} \mathrm{Er},{ }^{180} \mathrm{~W}$, and ${ }^{196} \mathrm{Hg}$, undergo $2 \mathrm{EC}$ processes. The lower energy release also results in a lower experimental sensitivity, since the background counting rate typically decreases with energy. In the following we overview the current status of experimental searches of the $2 \beta^{+}, \mathrm{EC} \beta^{+}$, and 2EC processes.

${ }^{36} \mathrm{Ar}$.- The first result for $0 \nu K L$ capture in ${ }^{36} \mathrm{Ar}$ $\left(T_{1 / 2}^{0 \nu} \geq 1.9 \times 10^{18} \mathrm{yr}\right)$ was obtained (Chkvorets, 2008) in the course of the research and development $(\mathrm{R} \& \mathrm{D})$ investigations in the GERDA experiment in the search for $0 \nu 2 \beta^{-}$ decay of ${ }^{76} \mathrm{Ge}$ using naked $\mathrm{HP}^{76} \mathrm{Ge}$ detectors in liquid argon. It was improved by 3 orders of magnitude with the data from phase I of the GERDA experiment in underground conditions of the Gran Sasso underground laboratory [LNGS, depth of 3600 m. (w.e.)] where 89.2 tons of liquid argon were used as the coolant medium and shield, $T_{1 / 2}^{0 \nu}(K L)>3.6 \times 10^{21} \mathrm{yr}$ at 90\% C.L. (Agostini et al., 2016).

${ }^{40} \mathrm{Ca}$.- - Limits for 2EC decay in ${ }^{40} \mathrm{Ca}$ were set in 1999 with the help of two $370 \mathrm{~g}$ low radioactive $\mathrm{CaF}_{2}(\mathrm{Eu})$ crystal scintillators at the LNGS over $\simeq 104 \mathrm{~d}$ (Belli et al., 1999). The low-energy threshold of $4 \mathrm{keV}$ allowed Belli et al. to also set a limit on the $2 \nu$ process, in which an energy release in the detector of only $6.4 \mathrm{keV}$ was expected. The achieved $T_{1 / 2}$ limits (approximately $10^{21} \mathrm{yr}$ ) were recently slightly improved with $\mathrm{CaWO}_{4}$ scintillating bolometers (total exposure of $730 \mathrm{~kg} \mathrm{~d}$ ) (Angloher et al., 2016), which were used in the CRESST-II dark-matter experiment.

${ }^{50} \mathrm{Cr}$. - The first limit on $\mathrm{EC} \beta^{+}$decay of ${ }^{50} \mathrm{Cr}$ was set with photographic emulsions to record tracks of $\beta$ particles (Fremlin and Walters, 1952). The measurements underground at a depth of $570 \mathrm{~m}$ gave a half-life limit of approximately $10^{14} \mathrm{yr}$. It was improved in 1985 with two HPGe detectors of $110 \mathrm{~cm}^{3}$ volume each, searching in coincidence for two $511 \mathrm{keV} \gamma$ quanta after annihilation of the emitted positron; measurements of a $148 \mathrm{~g} \mathrm{Cr}$ sample for $163 \mathrm{~h}$ resulted in the limit $T_{1 / 2}^{0 \nu+2 \nu}>1.8 \times 10^{17} \mathrm{yr}$ (Norman, 1985). The best current EC $\beta^{+}$sensitivity $\left(T_{1 / 2}>1.3 \times 10^{18} \mathrm{yr}\right)$ was achieved by Bikit et al. (2003) with a HPGe detector that measured in coincidence with a $\mathrm{NaI}(\mathrm{Tl})$ a $209 \mathrm{~g} \mathrm{CrO}_{3}$ sample for $720 \mathrm{~h}$.

${ }^{54} \mathrm{Fe}$. - The only known limits on double-electron capture in ${ }^{54} \mathrm{Fe}$ were set in the measurements of Bikit et al. (1998) with the help of a HPGe detector placed in the center of an iron cube with an inner volume of $1 \mathrm{~m}^{3}$ and a wall thickness of $25 \mathrm{~cm}$. The search for $\gamma$ rays emitted in the 2EC decay for $\simeq 6700 \mathrm{~h}$ gave $T_{1 / 2}$ limits of approximately $(4.1-5.0) \times$ $10^{20} \mathrm{yr}$, depending on the decay channel $(K K, K L$, or $L L)$.

${ }^{58} \mathrm{Ni}$. - The first limit on $\mathrm{EC} \beta^{+}$decay of ${ }^{58} \mathrm{Ni}$ of $\simeq 10^{17} \mathrm{yr}$ was reported in an experiment with photoemulsion plates (Fremlin and Walters, 1952). A level of $\lim T_{1 / 2} \sim 10^{19} \mathrm{yr}$ was reached in an experiment with a $\mathrm{Ge}(\mathrm{Li})$ detector and a $2.1 \mathrm{~kg} \mathrm{Ni}$ sample for $187 \mathrm{~h}$ (Bellotti et al., 1982), and in 
measurements with a $1.6 \mathrm{~kg} \mathrm{Ni}$ sample using two $\mathrm{NaI}(\mathrm{Tl})$ scintillators in coincidence for $100 \mathrm{~h}$ (Norman and DeFaccio, 1984). The sensitivity was improved by 1 order of magnitude in underground measurements at the Baksan Neutrino Observatory of $660 \mathrm{~m}$. (w.e.) depth with two $\mathrm{NaI}(\mathrm{Tl})$ scintillators and a $1.9 \mathrm{~kg} \mathrm{Ni}$ sample (Vasil'ev et al., 1993). Further improvement of the sensitivity to the neutrinoless $2 \mathrm{EC}$ process of $T_{1 / 2}>2.1 \times 10^{21} \mathrm{yr}$ was achieved by using a $7.3 \mathrm{~kg} \mathrm{Ni}$ sample measured for $1400 \mathrm{~h}$ with a low-background HPGe detector at the Felsenkeller underground laboratory $[110 \mathrm{~m}$. (w.e.)] (Lehnert, Degering et al., 2016). The best sensitivity to date was reported by Rukhadze et al. (2020), who used a large volume $\left(\approx 600 \mathrm{~cm}^{3}\right)$ HPGe detector and a $21.7 \mathrm{~kg}$ Ni sample installed at the Modane Underground Laboratory [4800 m. (w.e.)] to search for $\gamma$ quanta expected in the $2 \mathrm{EC}$ and $\mathrm{EC} \beta^{+}$ processes in ${ }^{58} \mathrm{Ni}$. A preliminary limit on the $0 \nu 2 \mathrm{EC}$ g.s. to g.s. transition in ${ }^{58} \mathrm{Ni}$ is $T_{1 / 2}>4.1 \times 10^{22} \mathrm{yr}$.

${ }^{64} \mathrm{Zn}$.- - Searches for ${ }^{64} \mathrm{Zn} \mathrm{EC} \beta^{+}$activity started in 1952 when a Zn sample was measured with photoemulsions (Fremlin and Walters, 1952). However, no useful limits could be extracted from these data because the emitted positrons have a low energy of $\leq 73 \mathrm{keV}$. In 1953, the characteristic $\mathrm{x}$ rays resulting from $2 \mathrm{EC}$ decay were searched for with proportional counters. The determined limit was approximately $10^{16}$ yr (Berthelot et al., 1953). In 1985, EC $\beta^{+}$decay of ${ }^{64} \mathrm{Zn}$ was searched for with two HPGe detectors with volume $110 \mathrm{~cm}^{3}$ each looking for $511 \mathrm{keV} \gamma$ rays in coincidence from a $228 \mathrm{~g} Z$ n sample. A limit $T_{1 / 2}^{0 \nu+2 \nu}>2.3 \times$ $10^{18} \mathrm{yr}$ was set after $161 \mathrm{~h}$ of measurements (Norman, 1985).

In 1995, an indication on a positive $\mathrm{EC} \beta^{+}$effect was claimed in measurements (at Earth's surface) using a $350 \mathrm{~g} \mathrm{Zn}$ sample with HPGe and $\mathrm{NaI}(\mathrm{Tl})$ detectors in coincidence. After nearly $400 \mathrm{~h}$ of data taking an excess of counts in the $511 \mathrm{keV}$ annihilation peak was observed that corresponds to a half-life $T_{1 / 2}^{0 \nu+2 \nu}=(1.1 \pm 0.9) \times 10^{19} \mathrm{yr}$ (Bikit et al., 1995). This claim was disproved in two experiments:

(1) Search for double-beta-plus processes in ${ }^{64} \mathrm{Zn}$ with emission of $\gamma$ quanta by using a HPGe detector (with volume $456 \mathrm{~cm}^{3}$ ) and a $\mathrm{CsI}(\mathrm{Tl})$ scintillator $\left(\simeq 400 \mathrm{~cm}^{3}\right)$ in coincidence. The measurements of a $460 \mathrm{~g} \mathrm{Zn}$ sample for $375 \mathrm{~h}$ at the Cheong-Pyung underground laboratory $[1000 \mathrm{~m}$. (w.e.) $]$ gave a limit $T_{1 / 2}^{0 \nu+2 \nu}>1.3 \times 10^{20} \mathrm{yr}$ (Kim et al., 2007).

(2) A scintillation detector with a $117 \mathrm{~g} \mathrm{ZnWO}_{4}$ crystal scintillator was utilized in an experiment at the LNGS for $1902 \mathrm{~h}$. $\mathrm{EC} \beta^{+}$decay was not observed, the limits $T_{1 / 2}^{0 \nu}>2.2 \times 10^{20} \mathrm{yr}$ and $T_{1 / 2}^{2 \nu}>2.1 \times 10^{20} \mathrm{yr}$ were set (Belli et al., 2008a). The result was further improved with a larger $0.7 \mathrm{~kg}$ detector to $T_{1 / 2}^{0 \nu+2 \nu} \geq$ $9 \times 10^{20}$ (Belli, Bernabei et al., 2011b).

The $2 \mathrm{EC}$ processes in ${ }^{64} \mathrm{Zn}$ were searched for in the following experiments:

(1) Half-life limits on the $2 \mathrm{EC}$ decay of ${ }^{64} \mathrm{Zn}$ of approximately $10^{17} \mathrm{yr}$ were set with the help of a $\mathrm{Cd}_{0.9} \mathrm{Zn}_{0.1} \mathrm{Te}$ semiconductor detector with mass $2.89 \mathrm{~g}$ over $1117 \mathrm{~h}$ of data taking at the LNGS (Kiel, Münstermann, and Zuber, 2003). The sensitivity was moderately improved in the next stage of the experiment with four
$\mathrm{Cd}_{0.9} \mathrm{Zn}_{0.1}$ Te crystals of $6.5 \mathrm{~g}$ each (Bloxham et al., 2007). Advancements in experimental sensitivity are expected with the currently running setup of 64 $\mathrm{Cd}_{0.9} \mathrm{Zn}_{0.1}$ Te detectors (Ebert et al., 2016b).

(2) $\mathrm{ZnWO}_{4}$ crystal scintillator, mass of $4.5 \mathrm{~g}, 429 \mathrm{~h}$ of data collection in the Solotvina Underground Laboratory $[1000 \mathrm{~m}$. (w.e.)], half-life limits of approximately $10^{18}$ yr (Danevich et al., 2005).

(3) $\mathrm{ZnWO}_{4}$ scintillators with mass up to $0.7 \mathrm{~kg}$ at the LNGS (Belli, Bernabei et al., 2011b). The strongest limits to date on the $2 \mathrm{EC}$ decay of ${ }^{64} \mathrm{Zn}$ were set: $T_{1 / 2}^{2 \nu} \geq 1.1 \times 10^{19} \mathrm{yr}$ and $T_{1 / 2}^{0 \nu} \geq 3.2 \times 10^{20} \mathrm{yr}$.

${ }^{74} \mathrm{Se}$. - The first search for $\mathrm{EC} \beta^{+}$processes in ${ }^{74} \mathrm{Se}$ was performed in measurements of a $563 \mathrm{~g}$ Se sample for $437 \mathrm{~h}$ with a $400 \mathrm{~cm}^{3}$ HPGe detector and resulted in $T_{1 / 2}$ limits of approximately $10^{18}-10^{19} \mathrm{yr}$ (Barabash, Hubert, Nachab, and Umatov, 2007), depending on the decay mode $(2 \nu$ or $0 \nu)$ and excited level of the daughter nucleus. The next search for a potentially resonant $L L$ capture of ${ }^{74} \mathrm{Se}$ to the $1204 \mathrm{keV}$ level of ${ }^{74} \mathrm{Ge}$ was realized in an experiment (Frekers et al., 2011) using HPGe $\gamma$ spectrometry of a $3 \mathrm{~kg}$ Se sample with a limit $T_{1 / 2} \geq 4.3 \times 10^{19} \mathrm{yr}$. However, this value was corrected to $>1.4 \times 10^{18}$ yr by Ješkovský et al. (2015) and reevaluated as $>3.9 \times 10^{16}$ yr by Lehnert, Wester et al. (2016) because the method of setting the limit was not reliable enough. Lehnert, Wester et al. (2016) obtained the limit (Ješkovský et al., 2015) for the $0 \nu 2 \mathrm{EC}$ transition of ${ }^{74} \mathrm{Se}$ to the $1204 \mathrm{keV}$ excited level of ${ }^{74} \mathrm{Ge}, T_{1 / 2} \geq 1.5 \times 10^{19} \mathrm{yr}$ with the help of HPGe $\gamma$ spectrometry in coincidence with $\mathrm{NaI}(\mathrm{Tl})$ scintillation counter of the same selenium sample as in the experiment (Frekers et al., 2011). The limit was reevaluated as $T_{1 / 2} \geq$ $6.3 \times 10^{17} \mathrm{yr}$ for the same reason of a not quite correct interpretation of the experiment sensitivity as a half-life limit. Lehnert, Wester et al. (2016), after measurements of an $\approx 2.5 \mathrm{~kg}$ selenium sample by using the HPGe $\gamma$ detector located in the Felsenkeller underground laboratory, obtained $T_{1 / 2} \geq 7.0 \times 10^{18} \mathrm{yr}$ for the $0 \nu L L$ transition of ${ }^{74} \mathrm{Se}$ to the $1204.2 \mathrm{keV}$ excited state of ${ }^{74} \mathrm{Ge}$. This value was further improved recently with $600 \mathrm{~cm}^{3} \mathrm{HPGe}$ detector and $1.6 \mathrm{~kg} \mathrm{Se}$ source to $T_{1 / 2} \geq 1.1 \times 10^{19} \mathrm{yr}$, with $\simeq 10^{19} \mathrm{yr}$ limits also for other transitions (Barabash et al., 2020).

${ }^{78} \mathrm{Kr} .-{ }^{78} \mathrm{Kr}$ is one of the six nuclides whose $Q$ values allow all three channels of double-beta-plus decays: $2 \mathrm{EC}, \mathrm{EC} \beta^{+}$, and $2 \beta^{+}$. The first experiment to search for $\mathrm{EC} \beta^{+}$and $2 \beta^{+}$ decay of ${ }^{78} \mathrm{Kr}$ was performed in 1994 with a high pressure ionization chamber placed within an array of six large volume $\mathrm{NaI}(\mathrm{Tl})$ scintillators of $\simeq 3000 \mathrm{~cm}^{3}$ each. The ionization chamber contained $35 \mathrm{lt}$ of enriched to $94.08 \%{ }^{78} \mathrm{Kr}$. The data were collected in the Canfranc Underground Laboratory [675 m. (w.e.)] for $4435 \mathrm{~h}$; derived $T_{1 / 2}$ limits for $2 \beta^{+}$and $\mathrm{EC} \beta^{+}$processes remain among the best reached for doublebeta-plus nuclei $\left(\simeq 10^{21} \mathrm{yr}\right)$ (Saenz et al., 1994).

Experiments (Gavriljuk et al., 2000, 2011) at the Baksan Neutrino Observatory [4900 m. (w.e.)] with enriched ${ }^{78} \mathrm{Kr}$ led to limits of approximately $10^{20}-10^{21} \mathrm{yr}$. An indication of the $2 \nu K K$ capture with $T_{1 / 2}=9.2_{-2.9}^{+5.7} \times 10^{21} \mathrm{yr}$ was obtained with a large proportional counter (49 lt) filled by gas enriched in ${ }^{78} \mathrm{Kr}$ to $99.81 \%$ (Gavrilyuk et al., 2013) [recently updated to 
$1.9_{-0.8}^{+1.3} \times 10^{22} \mathrm{yr}$ (Ratkevich et al., 2017)]. Limits for other modes of decay were also set: in particular, a possible resonant $0 \nu K K$ capture to the $2^{+} 2838 \mathrm{keV}$ level of ${ }^{78} \mathrm{Se}$ was limited as $T_{1 / 2} \geq 5.4 \times 10^{21} \mathrm{yr}$.

${ }^{84} \mathrm{Sr}$.-The first limit $\left(\simeq 10^{13} \mathrm{yr}\right)$ on $\mathrm{EC} \beta^{+}$decay of ${ }^{84} \mathrm{Sr}$ was derived by Tretyak and Zdesenko (1995) through analysis of the photoemulsion experiment (Fremlin and Walters, 1952) with corrections on the decay energy and the natural abundance of the isotope. $2 \mathrm{EC}$ and $\mathrm{EC} \beta^{+}$processes in ${ }^{84} \mathrm{Sr}$ have been investigated at a level of $T_{1 / 2} \sim 10^{15}-10^{16} \mathrm{yr}$ with a small (6.6 g) sample of $\operatorname{SrI}_{2}(\mathrm{Eu})$ crystal scintillator measured over $706 \mathrm{~h}$ at the LNGS using a $468 \mathrm{~cm}^{3}$ HPGe detector (Belli et al., 2012a). Furthermore, $\mathrm{R} \& \mathrm{D}$ with a $54 \mathrm{~g} \mathrm{SrF}_{2}$ scintillating bolometer (Diaz, 2013) allowed limits to be set on a few decay modes to $T_{1 / 2} \sim 10^{15}-10^{16} \mathrm{yr}$.

${ }^{92} \mathrm{Mo}$.- A limit on EC $\beta^{+}$decay of ${ }^{92} \mathrm{Mo}$ can be extracted from a photoemulsion experiment (Fremlin and Walters, $1952)$ as $\simeq 10^{15}$ yr. In 1955 , double-positron tracks emitted from a Mo foil were searched for with a Wilson cloud chamber (Winter, 1955b). The determined limit of $4 \times 10^{18} \mathrm{yr}$ should be discarded because, as we now know, the energy released in the decay is not sufficient for emission of two positrons. The search for the 2EC transition of ${ }^{92} \mathrm{Mo}$ to excited levels of ${ }^{92} \mathrm{Zr}$ was realized in 1982 in measurements on Earth's surface using a $1.82 \mathrm{~kg}$ Mo sample with a $130 \mathrm{~cm}^{3} \mathrm{Ge}(\mathrm{Li})$ detector for $161 \mathrm{~h}$, with limits of approximately $10^{18} \mathrm{yr}$ (Bellotti et al., 1982). In 1985, a $324 \mathrm{~g}$ Mo sample was sandwiched between two HPGe detectors of $110 \mathrm{~cm}^{3}$ each looking for coincidence of $511 \mathrm{keV} \gamma$ quanta; $T_{1 / 2}$ limits of $\simeq 10^{17} \mathrm{yr}$ were set (Norman, 1985). In 1987, positrons emitted from thin Mo foil in $\mathrm{EC} \beta^{+}$ decay of ${ }^{92} \mathrm{Mo}$ were searched for with a time projection chamber; after $3.05 \mathrm{~h}$ of data taking, limits were set as $10^{17}-10^{18}$ yr (Elliott, Hann, and Moe, 1987). The best known limits on the double-beta decay of ${ }^{92} \mathrm{Mo}$ of approximately $10^{21} \mathrm{yr}$ were set in 1995-1997 in underground measurements of $2.5 \mathrm{~kg}$ monocrystal Mo rods with a $400 \mathrm{~cm}^{3}$ HPGe detector in the Modane Underground Laboratory for $1316 \mathrm{~h}$ (Aunola et al., 1995; Barabash et al., 1997).

The search for $\mathrm{EC} \beta^{+}$decay of ${ }^{92} \mathrm{Mo}$ was also pursued in the Yangyang Underground Laboratory at a depth of $700 \mathrm{~m}$ with a $277 \mathrm{~g} \mathrm{CaMoO}_{4}$ crystal scintillator surrounded by 14 large CsI scintillators that served as a passive and active shielding (Lee et al., 2011); the achieved limit of $T_{1 / 2} \geq 2.3 \times 10^{20} \mathrm{yr}$ is slightly better than the result from Barabash et al. (1997) $\left(T_{1 / 2} \geq 1.9 \times 10^{20} \mathrm{yr}\right)$. The sensitivity of the experiment by Kang et al. (2013), in which a $411 \mathrm{~g} \mathrm{CaMoO}_{4}$ crystal was used in coincidence with a HPGe detector, was approximately $10^{19}-10^{20} \mathrm{yr}$.

${ }^{96} \mathrm{Ru}$. - In fact, ${ }^{96} \mathrm{Ru}$ is the only nuclide from the list of six potential $2 \beta^{+}$decaying nuclides that has an appreciable natural isotopic abundance of $l=5.54 \%$. Notwithstanding these favorable features for experimental investigation, the first search was performed only in 1985, where a Ru sample of $50 \mathrm{~g}$ was measured with two HPGe detectors $\left(110 \mathrm{~cm}^{3}\right)$ in coincidence for $178 \mathrm{~h}$. Determined limits on $2 \beta^{+}$and EC $\beta^{+}$ decays to the ground state and excited levels of ${ }^{96} \mathrm{Mo}$ were about $10^{16} \mathrm{yr}$ (Norman, 1985). These limits were improved to approximately $\simeq 10^{19}$ yr by Belli et al. (2009b) and Andreotti et al. (2012). The best results of approximately $10^{20}-10^{21} \mathrm{yr}$ were obtained at the LNGS in measurements with a highly purified Ru sample (mass of $720 \mathrm{~g}$ ) and four HPGe detectors of $\simeq 225 \mathrm{~cm}^{3}$ each for $5479 \mathrm{~h}$ (Belli et al., 2013b).

${ }^{102} \mathrm{Pd}$.-First $T_{1 / 2}$ limits on $2 \mathrm{EC}$ processes in ${ }^{102} \mathrm{Pd}$ were obtained in the experiment at the Felsenkeller underground laboratory, where a $802 \mathrm{~g}$ Pd sample was measured with a HPGe detector; the results were on the order of $10^{18} \mathrm{yr}$ (Lehnert and Zuber, 2011). They were improved in measurements with two sandwiched HPGe detectors at the HADES underground laboratory [500 m. (w.e.)] (Lehnert et al., 2013). Further measurements were performed at the LNGS in a setup with four HPGe detectors ( $\simeq 225 \mathrm{~cm}^{3}$ each) for $2093 \mathrm{~h}$; joint analysis of all three measurements pushed the $T_{1 / 2}$ limits to approximately $10^{19} \mathrm{yr}$ (Lehnert, Andreotti et al., 2016).

${ }^{106} \mathrm{Cd}$. - The nuclide ${ }^{106} \mathrm{Cd}$ is among the most investigated $2 \beta^{+}$nuclides, with a long history of experimental studies. Limits on $2 \beta^{+}$and $\mathrm{EC} \beta^{+}$decays of approximately $10^{15} \mathrm{yr}$ can be extracted from the measurements using a $\mathrm{Cd}$ sample with photographic emulsions. Searches for positrons emitted in $2 \beta^{+}$decay were performed in 1955 with a Wilson cloud chamber in the magnetic field and a $\mathrm{Cd}$ foil with a mass of $30 \mathrm{~g}$; the experiment resulted in a limit of $\simeq 10^{16} \mathrm{yr}$ (Winter, 1955b). In 1984, in measurements of a $153 \mathrm{~g} \mathrm{Cd}$ sample for $72 \mathrm{~h}$ with two $\mathrm{NaI}(\mathrm{Tl})$ scintillators in coincidence, limits $\simeq 10^{17} \mathrm{yr}$ were determined for $2 \beta^{+}, \mathrm{EC} \beta^{+}$and $2 \mathrm{EC}$ processes (Norman and DeFaccio, 1984).

An experiment with a ${ }^{116} \mathrm{CdWO}_{4}$ crystal scintillator $\left(15 \mathrm{~cm}^{3}\right.$, enriched in ${ }^{116} \mathrm{Cd}$ to $83 \%$ ) in the Solotvina Underground Laboratory was aimed at the investigation of $2 \beta^{-}$decay of ${ }^{116} \mathrm{Cd}$. However, the data collected over $2982 \mathrm{~h}$ were also used to set limits for other nuclides, in particular, for ${ }^{106} \mathrm{Cd}$ of approximately $10^{17}-10^{19} \mathrm{yr}$, depending on the decay mode Georgadze et al. (1995). A "source = detector" approach and a low-energy threshold allowed a limit to be set on $2 \nu K K$ capture $\left(\simeq 10^{17} \mathrm{yr}\right)$ for the first time.

An external Cd foil $(331 \mathrm{~g})$ was measured at the Modane Underground Laboratory with a $120 \mathrm{~cm}^{3}$ HPGe detector for $1137 \mathrm{~h}$, aiming at the detection of $\gamma$ quanta from annihilation of positrons and from deexcitation of the daughter ${ }^{106} \mathrm{Pd}$ nucleus if the excited levels are populated (Barabash et al., 1996). Half-life limits of approximately $10^{18}-10^{19} \mathrm{yr}$ were set. In 1996, a large $(1.046 \mathrm{~kg}) \mathrm{CdWO}_{4}$ scintillator with natural Cd composition was measured at the LNGS for $6701 \mathrm{~h}$ (Danevich et al., 1996). Limits on the half-life for $2 \beta^{+}$and $\mathrm{EC} \beta^{+}$decays were about $\simeq 10^{19} \mathrm{yr}$ for $0 \nu$, and limits were approximately $\simeq 10^{17} \mathrm{yr}$ for $2 \nu$ processes. A small $(0.5 \mathrm{~g})$ CdTe bolometer operating at a temperature of $10 \mathrm{mK}$ was tested for $72 \mathrm{~h}$ by Ito et al. (1997). The derived limit on $0 \nu \mathrm{EC} \beta^{+}$decay of ${ }^{106} \mathrm{Cd}$ was $1.4 \times 10^{16} \mathrm{yr}$, which is lower than those reached with more traditional techniques at that time, particularly due to the small size of the CdTe bolometer.

$\mathrm{Cd}$ samples of natural composition with $1.25 \%$ of ${ }^{106} \mathrm{Cd}$ were used in all the previously mentioned experiments [in a ${ }^{116} \mathrm{CdWO}_{4}$ scintillator (Georgadze et al., 1995$){ }^{106} \mathrm{Cd}$ was even depleted]. On the contrary, a cadmium sample with mass of $154 \mathrm{~g}$ enriched by ${ }^{106} \mathrm{Cd}$ in $68 \%$ was used in experiment (Belli, Bernabei et al., 1999). Measurements at the LNGS with 
two low-background $\mathrm{NaI}(\mathrm{Tl})$ scintillators for 4321 h enabled a level of the half-life sensitivity of more than $10^{20} \mathrm{yr}$ to be reached for $2 \beta^{+}, \mathrm{EC} \beta^{+}$, and $2 \mathrm{EC}$ processes accompanied by emission of $\gamma$ quanta. In 2003, the long-term (14183 h) experiment in the Solotvina Underground Laboratory with three enriched ${ }^{116} \mathrm{CdWO}_{4}$ scintillators (total mass of $330 \mathrm{~g}$ ) was finished (Danevich et al., 2003). Together with measurements for $433 \mathrm{~h}$ with a nonenriched $\mathrm{CdWO}_{4}$ crystal of $454 \mathrm{~g}$, sensitivity for ${ }^{106} \mathrm{Cd}$ was improved by approximately 1 order of magnitude over the result of Georgadze et al. (1995).

A radiopure cadmium tungstate crystal scintillator with a mass of $216 \mathrm{~g}$ produced from cadmium enriched in ${ }^{106} \mathrm{Cd}$ to $66 \%\left({ }^{106} \mathrm{CdWO}_{4}\right)$ was grown by Belli et al. (2010). Measurements at the LNGS gave limits on $2 \beta$ processes of approximately $10^{20} \mathrm{yr}$ (Belli et al., 2012b). In the second stage, the ${ }^{106} \mathrm{CdWO}_{4}$ scintillator was installed between four HPGe detectors ( $\simeq 225 \mathrm{~cm}^{3}$ each) to improve the sensitivity to decay modes accompanied by $\gamma$ quanta; this resulted in limits of $T_{1 / 2} \geq 10^{20}-10^{21} \mathrm{yr}$ (Belli et al., 2016). In the third stage, the ${ }^{106} \mathrm{CdWO}_{4}$ detector was running in coincidence with two large $\mathrm{CdWO}_{4}$ crystal scintillators in a close geometry to increase the detection efficiency of $\gamma$ quanta emitted from the ${ }^{106} \mathrm{CdWO}_{4}$ crystal. The sensitivity of the experiment is approaching the theoretical predictions for the $2 \nu \mathrm{EC} \beta^{+}$decay of ${ }^{106} \mathrm{Cd}$ (that are in the interval $10^{21}-10^{22} \mathrm{yr}$ ). The resonant neutrinoless doubleelectron capture to the $2718 \mathrm{keV}$ excited state of ${ }^{106} \mathrm{Pd}$ is restricted as $T_{1 / 2} \geq 2.9 \times 10^{21} \mathrm{yr}$ (Belli et al., 2020).

At present, there are two other running experiments probing the double-beta decays of ${ }^{106} \mathrm{Cd}$ : COBRA and TGV-2. The COBRA experiment started at the LNGS with two small semiconductor detectors: $\mathrm{Cd}_{0.9} \mathrm{Zn}_{0.1} \mathrm{Te}$ (mass of $\simeq 3 \mathrm{~g}$ ) and CdTe (mass of $\simeq 6 \mathrm{~g}$ ) (Kiel, Münstermann, and Zuber, 2003). CdZnTe crystals are used in the current stage (Ebert et al., 2013, 2016a). Results for ${ }^{106} \mathrm{Cd} T_{1 / 2}$ limits are approximately $10^{18} \mathrm{yr}$. The main aim of the TGV-2 experiment (located at the Modane Underground Laboratory) is the search for $2 \nu K K$ decay of ${ }^{106} \mathrm{Cd}$ (this channel has the lowest expected half-life). In the experiment, 32 planar HPGe detectors are used with a total sensitive volume $\approx 400 \mathrm{~cm}^{3}$; see Fig. 11 . The Cd foils were enriched in ${ }^{106} \mathrm{Cd}$ to $60 \%-75 \%$ in the first stage of the experiment (Rukhadze et al., 2006, 2011a, 2011b); $23.2 \mathrm{~g}$ of Cd enriched to $99.57 \%$ of ${ }^{106} \mathrm{Cd}$ were used in Rukhadze et al. (2016). After $\simeq 8200 \mathrm{~h}$ of data taking, limits are of approximately $10^{20} \mathrm{yr}$.

${ }^{108} \mathrm{Cd}$. - The first limits $\left(10^{16}-10^{17} \mathrm{yr}\right)$ on $2 \mathrm{EC}$ decay of ${ }^{108} \mathrm{Cd}$ were determined in 1995 , with a ${ }^{116} \mathrm{CdWO}_{4}$ scintillator in the Solotvina Underground Laboratory (Georgadze et al., 1995). They were improved later to more than $10^{17} \mathrm{yr}$ with higher statistics collected $(14183 \mathrm{~h})$ and with data of the larger (454 g) $\mathrm{CdWO}_{4}$ scintillator with natural $\mathrm{Cd}$ composition (Danevich, Georgadze et al., 1996; Danevich et al., 2003). The best limits on the $2 \mathrm{EC}$ decay $\left(\simeq 10^{18} \mathrm{yr}\right)$ were obtained at the LNGS in the COBRA experiment (Kiel, Münstermann, and Zuber, 2003) and with a $\mathrm{CdWO}_{4}$ crystal scintillator of $434 \mathrm{~g}$ measured for $2758 \mathrm{~h}$ in the lowbackground DAMA/R\&D setup (Belli et al., 2008b).

${ }^{112} \mathrm{Sn}$. - The first limit on the $0 \nu \mathrm{EC} \beta^{+}$decay of ${ }^{112} \mathrm{Sn}$ of approximately $\simeq 10^{13} \mathrm{yr}$ was derived by Tretyak and
Zdesenko (1995) from the measurements of Sn samples with photographic emulsions (Fremlin and Walters, 1952). In 2007 bounds on $\mathrm{EC} \beta^{+}$processes in ${ }^{112} \mathrm{Sn}$ were improved to $\simeq 10^{18} \mathrm{yr}$ in an $\mathrm{R} \& \mathrm{D}$ to develop tin-loaded scintillators for $2 \beta$ experiments with ${ }^{112} \mathrm{Sn}$ and ${ }^{124} \mathrm{Sn}$. A 1 lt sample of tetrabutyltin $\left(\mathrm{C}_{4} \mathrm{H}_{9}\right)_{4} \mathrm{Sn}$ with a $\mathrm{Sn}$ concentration of $34 \%$ was measured for $140 \mathrm{~h}$ with a $456 \mathrm{~cm}^{3}$ HPGe detector in the Cheong-Pyung underground laboratory (Kim et al., 2007). In another experiment, a $72 \mathrm{~cm}^{3}$ Ge detector was used for measurements of a $1.24 \mathrm{~kg}$ Sn sample on Earth's surface for $831 \mathrm{~h}$ (Dawson, Ramaswamy et al., 2008). For 2EC processes with a population of the ground and excited states of ${ }^{112} \mathrm{Cd}$, limits were about $10^{18} \mathrm{yr}$.

In a series of measurements by Barabash et al. (2008, 2009), Dawson, Degering et al. (2008), and Kidd, Esterline, and Tornow (2008) performed mostly underground with HPGe detectors and external $\mathrm{Sn}$ sources (with natural composition and enriched in ${ }^{112} \mathrm{Sn}$ ), limits of approximately $10^{19}-10^{20} \mathrm{yr}$ were obtained. Limits in the range of $(0.1-1.6) \times 10^{21} \mathrm{yr}$ were achieved with a $100 \mathrm{~g} \mathrm{Sn}$ sample enriched in ${ }^{112} \mathrm{Sn}$ to $94.32 \%$ measured in the Modane Underground Laboratory with a $380 \mathrm{~cm}^{3}$ HPGe detector for $3175 \mathrm{~h}$ (Barabash et al., 2011).

${ }^{120} \mathrm{Te}$. - The first limit on the $0 \nu \mathrm{EC} \beta^{+}$decay of ${ }^{120} \mathrm{Te}$ $\left(\simeq 10^{12} \mathrm{yr}\right)$ was determined by Tretyak and Zdesenko (1995) on the basis of the measurements of Te samples with photoemulsions (Fremlin and Walters, 1952). It was improved to $\simeq 10^{16}$ yr by the COBRA Collaboration with small semiconductor detectors $\mathrm{Cd}_{0.9} \mathrm{Zn}_{0.1} \mathrm{Te}(\simeq 3 \mathrm{~g}, 1117 \mathrm{~h}$ of data collection) and $\mathrm{CdTe}(\simeq 6 \mathrm{~g}, 1645 \mathrm{~h})$ in the LNGS (Kiel, Münstermann, and Zuber, 2003). Limits on $K K$ captures to the ground state and the first excited level of ${ }^{120} \mathrm{Sn}$ were set at about $10^{16} \mathrm{yr}$ (Kiel, Münstermann, and Zuber, 2003). Somewhat improved results were attained by Bloxham et al. (2007) and Dawson et al. (2009a). The 2EC limits were further improved to $10^{17}-10^{18} \mathrm{yr}$ by measuring $1 \mathrm{~kg}$ of natural $\mathrm{TeO}_{2}$ powder with a $400 \mathrm{~cm}^{3} \mathrm{HPGe}$ detector for $475 \mathrm{~h}$ at the Modane Underground Laboratory (Barabash, Hubert, Hubert, and Umatov, 2007). The best results for the $\mathrm{EC} \beta^{+}$mode were obtained in the CUORICINO/CUORE-0 experiments at the LNGS with $\mathrm{TeO}_{2}$ bolometers: $T_{1 / 2}>2.7 \times 10^{21} \mathrm{yr}$ for $0 \nu \mathrm{EC} \beta^{+}$(Alduino et al., 2018b) and $T_{1 / 2}>7.6 \times 10^{19} \mathrm{yr}$ for $2 \nu \mathrm{EC} \beta^{+}$(Andreotti et al., 2011).

${ }^{124} \mathrm{Xe} .-{ }^{124} \mathrm{Xe}$ has the highest available energy that can be released in $2 \beta^{+}$processes $(2864 \mathrm{keV})$. In the first experiment with a gridded ionization chamber (volume of $3.66 \mathrm{lt}$ ), filled with $\mathrm{Xe}$ and installed at the Baksan Neutrino Observatory 850 m. (w.e.) underground, the Xe gas of natural composition and Xe sample enriched in ${ }^{136} \mathrm{Xe}$ to $93 \%$ were measured over 710 and $685 \mathrm{~h}$, respectively. Limits on the $2 \beta^{+}$and $\mathrm{EC} \beta^{+}$ decays of ${ }^{124} \mathrm{Xe}$ were set in the range of $10^{14}-10^{18} \mathrm{yr}$, depending on the decay mode (Barabash et al., 1989). A limit on $2 \nu K K$ decay was set by using a multiwire wall-less proportional counter (fiducial volume of $4.44 \mathrm{lt}$, working pressure of $4.8 \mathrm{~atm}$ ), also installed at the Baksan Neutrino Observatory but at a larger depth of $4700 \mathrm{~m}$. (w.e.). After the measurements for $\simeq 1600 \mathrm{~h}$ with $\mathrm{Xe}$ samples of different isotopic composition, a limit on $2 \nu K K$ decay of ${ }^{124} \mathrm{Xe}$ was set at $T_{1 / 2} \geq 1.1 \times 10^{17} \mathrm{yr}$ (Gavriljuk et al., 1998). 
Recent developments and the start of the operation of massive dark-matter detectors based on Xe allowed limits to be extracted on $2 \nu K K$ decay of ${ }^{124} \mathrm{Xe}: T_{1 / 2}>2.1 \times 10^{22} \mathrm{yr}$ from $832 \mathrm{~kg}$ of liquid $\mathrm{Xe}$ at the Kamioka Observatory in Japan [2700 m. (w.e.)] measured for $19200 \mathrm{~h}$ (Abe et al., 2018) and $T_{1 / 2}>6.5 \times 10^{20} \mathrm{yr}$ from the XENON100 TPC with $62 \mathrm{~kg}$ of liquid Xe measured at the LNGS for $5390 \mathrm{~h}$ (Aprile et al., 2017). In both these experiments natural Xe was used, with a ${ }^{124} \mathrm{Xe}$ abundance of $\iota=0.095 \%$. The result from the Baksan Neutrino Observatory in Russia [4900 m. (w.e.)] obtained with $52 \mathrm{~g}$ of $\mathrm{Xe}$ enriched in ${ }^{124} \mathrm{Xe}$ to $21 \%$ after $1800 \mathrm{~h}$ of measurement with a copper proportional counter is also known: $T_{1 / 2}>2.5 \times 10^{21} \mathrm{yr}$ (Gavrilyuk et al., 2015). The $2 \nu 2 \mathrm{EC}$ decay in ${ }^{124} \mathrm{Xe}$ with a half-life of $T_{1 / 2}=(1.8 \pm 0.5) \times$ $10^{22} \mathrm{yr}$ was finally observed in 2019 using the XENON1T dark-matter detector (XENON Collaboration, 2019).

${ }^{126} \mathrm{Xe}$.- The limit on the ${ }^{126} \mathrm{Xe} 2 \nu K K$ decay was derived in the XMASS experiment: $T_{1 / 2}>1.9 \times 10^{22} \mathrm{yr}$ (Abe et al., 2018).

${ }^{130} \mathrm{Ba}$.- The first limits were derived as approximately $10^{11} \mathrm{yr}$ for the $2 \beta^{+}$decay and $10^{12} \mathrm{yr}$ for the $\mathrm{EC} \beta^{+}$decay (Tretyak and Zdesenko, 1995) from the experiment with photographic emulsions (Fremlin and Walters, 1952). In 1996, by reanalyzing the old geochemical measurements (Srinivasan, 1976 ) with respect to the amount of the daughter nuclide ${ }^{130} \mathrm{Xe}$ accumulated in a $\mathrm{BaSO}_{4}$ sample during geological time, the limit of $T_{1 / 2}>4 \times 10^{21} \mathrm{yr}$ was set for all modes of ${ }^{130} \mathrm{Ba}$ decay (Barabash and Saakyan, 1996). We note that an indication of the effect with $T_{1 / 2}=2.1_{-0.8}^{+3.0} \times 10^{21} \mathrm{yr}$ was obtained for another sample (Barabash and Saakyan, 1996). A claim of a positive observation of the ${ }^{130} \mathrm{Ba}$ decay with the half-life $T_{1 / 2}=(2.16 \pm$ $0.52) \times 10^{21} \mathrm{yr}$ (any decay channel and mode) was reported by Meshik et al. (2001) through analysis of ${ }^{130} \mathrm{Xe}$ excess in $\mathrm{a} \mathrm{BaSO}_{4}$ sample. It should be stressed that the $T_{1 / 2}$ value is consistent with the theoretical estimates for the $2 \nu K K$ decay of ${ }^{130} \mathrm{Ba}$. The excess of ${ }^{130} \mathrm{Xe}$ was also found in another geochemical experiment (Pujol et al., 2009), but the obtained half-life was smaller: $T_{1 / 2}=(6.0 \pm 1.1) \times 10^{20} \mathrm{yr}$. Meshik and Pravdivtseva (2017) explained this disagreement as the contribution from cosmogenics, and the result of Meshik et al. (2001) was considered more reliable.

As for the direct experiments with ${ }^{130} \mathrm{Ba}$, only one experiment was performed in 2004. $\mathrm{A} \mathrm{BaF}_{2}$ crystal scintillator with a mass of $3615 \mathrm{~g}$ was measured in coincidence with two $\mathrm{NaI}(\mathrm{Tl})$ detectors for $4253 \mathrm{~h}$ at the LNGS (Cerulli et al., 2004). Derived limits on $2 \beta^{+}$and $\mathrm{EC} \beta^{+}$decays to the ground state and few excited levels of ${ }^{130} \mathrm{Xe}$ are approximately $10^{17} \mathrm{yr}$, far from what is needed to check the possible positive claims of Meshik et al. (2001) and Pujol et al. (2009).

${ }^{132} \mathrm{Ba}$.- The first limit for $2 \mathrm{EC}$ in ${ }^{132} \mathrm{Ba} T_{1 / 2}>3.0 \times 10^{20} \mathrm{yr}$ was set by Barabash and Saakyan (1996) from the reanalysis of the geochemical data on excess of ${ }^{132} \mathrm{Xe}$ in $\mathrm{BaSO}_{4}$ samples (Srinivasan, 1976). An excess of ${ }^{132} \mathrm{Xe}$ was observed in the most "promising" barite sample in the already mentioned geochemical measurements (Meshik et al., 2001) (in the other four samples the ${ }^{132} \mathrm{Xe}$ excess was too large), leading to the ${ }^{132} \mathrm{Ba}$ half-life of $T_{1 / 2}=(1.3 \pm 0.9) \times 10^{21} \mathrm{yr}$. However, for ${ }^{132} \mathrm{Ba}$ Meshik et al. preferred more cautiously to give a limit of $T_{1 / 2}>2.2 \times 10^{21} \mathrm{yr}$.
${ }^{136} \mathrm{Ce}$. - The first limits on $2 \beta^{+}$decay of ${ }^{136} \mathrm{Ce}$ were obtained with the help of two $\mathrm{CeF}_{3}$ crystal scintillators (masses of 75 and $345 \mathrm{~g}$ ) measured for 88 and $693 \mathrm{~h}$, respectively, in the low-background setup at the LNGS. In particular, a sensitivity to the $0 \nu 2 \beta^{+}$decay was approximately $T_{1 / 2}>10^{18}$ yr (Bernabei et al., 1997).

Limits for the $\mathrm{EC} \beta^{+}$and $2 \mathrm{EC}$ processes in ${ }^{136} \mathrm{Ce}$ were derived from the long-term (13949 h) measurements with a $635 \mathrm{~g} \mathrm{Gd}_{2} \mathrm{SiO}_{5}(\mathrm{Ce})$ crystal scintillator in the Solotvina Underground Laboratory; the results for $T_{1 / 2}$ limits were approximately $10^{13}-10^{16} \mathrm{yr}$, depending on the decay mode (Danevich et al., 2001). The limit on $2 \nu K K$ capture was later improved from $\simeq 10^{13}$ to $\simeq 10^{16}$ yr in measurements with a $\mathrm{CeF}_{3}$ scintillation detector $(49 \mathrm{~g}$ ) for $2142 \mathrm{~h}$ at the LNGS (Belli et al., 2003). A small (6.9 g) $\mathrm{CeCl}_{3}$ scintillator was measured at the LNGS for $1638 \mathrm{~h}$ also in the source $=$ detector approach, resulting in half-life limits of approximately $10^{16}-10^{17}$ yr (Belli, Bernabei et al., 2011a).

Measurements of Ce-containing materials as external targets with HPGe detectors at the LNGS $\left(\mathrm{CeCl}_{3} 6.9 \mathrm{~g}, \mathrm{HPGe}\right.$ $244 \mathrm{~cm}^{3}, 1280 \mathrm{~h}$ ) (Belli, Bernabei et al., 2009) and $\mathrm{CeO}_{2}$ [732 g, HPGe $465 \mathrm{~cm}^{3}, 1900 \mathrm{~h}$ (Belli et al., 2014)] led to limits of $\simeq 10^{17}-10^{18} \mathrm{yr}$ for modes accompanied by emission of $\gamma$ quanta. The last sample was additionally purified (the Th content in a $627 \mathrm{~g}$ sample was reduced by a factor of 60) and remeasured for $2299 \mathrm{~h}$ with the same detector. This led to an improvement of $T_{1 / 2}$ limits of approximately $>10^{18} \mathrm{yr}$ (Belli et al., 2017).

${ }^{138} \mathrm{Ce}$.- In the previously described experiments (Danevich et al., 2001; Belli et al., 2003, 2014; Belli, Bernabei et al., 2009, 2011a), the $2 \mathrm{EC}$ decays in ${ }^{138} \mathrm{Ce}$ were searched for too; the best limits to date on the neutrinoless $K K, K L$, and $L L$ decays are approximately $(4.0-5.5) \times 10^{17} \mathrm{yr}$ (Belli et al., 2017).

${ }^{144} \mathrm{Sm}$.- The first limit on the $2 \beta$ decay of ${ }^{144} \mathrm{Sm}$ $\left(\simeq 8 \times 10^{8}\right.$ yr $)$ was obtained by Nozzoli (2018) by analyzing the average abundances of parent-daughter nuclei in Earth's crust. The first counting experiment to search for double-beta processes in ${ }^{144} \mathrm{Sm}$ using low-background HPGe $\gamma$ spectrometry was performed at the LNGS by using a highly purified samarium oxide sample, with the limits on different channels and modes of $2 \mathrm{EC}$ and $\mathrm{EC} \beta^{+}$decays of approximately $T_{1 / 2} \geq$ $(0.13-1.3) \times 10^{20} \mathrm{yr}$ (Belli et al., 2019a).

${ }^{152} \mathrm{Gd}$. - The first limit on $2 \mathrm{EC}$ in ${ }^{152} \mathrm{Gd}$ was obtained similarly to that of ${ }^{144} \mathrm{Sm}$ as $T_{1 / 2}>6 \times 10^{8} \mathrm{yr}$ (Nozzoli, 2018).

${ }^{156} \mathrm{Dy}$.- The first searches for double-beta processes in ${ }^{156}$ Dy were realized at the LNGS through measurements of a $322 \mathrm{~g}$ sample of dysprosium oxide $\mathrm{Dy}_{2} \mathrm{O}_{3}$ of $99.98 \%$ purity grade with a HPGe detector $\left(244 \mathrm{~cm}^{3}\right)$ for $2512 \mathrm{~h}$ (Belli et al., 2011a). The limits obtained were about $\simeq 10^{14}-10^{16} \mathrm{yr}$, depending on the decay mode. Finch and Tornow (2015) measured two enriched $\mathrm{Dy}_{2} \mathrm{O}_{3}$ sources $(803 \mathrm{mg}$, enriched in ${ }^{156} \mathrm{Dy}$ to $21.58 \%$, and $344 \mathrm{mg}, 20.9 \%$ ) at the Kimballton Underground Research Facility in Virginia [1450 m. (w.e.)] with two HPGe detectors in coincidence. The $T_{1 / 2}$ limits were improved to approximately $\simeq 10^{17}-10^{18} \mathrm{yr}$.

${ }^{158} \mathrm{Dy}$. - The limits on the $2 \mathrm{EC}$ processes in ${ }^{158} \mathrm{Dy}$ of approximately $T_{1 / 2}>(0.35-1.0) \times 10^{15} \mathrm{yr}(2 \nu$ mode $)$ and 
$T_{1 / 2}>(0.026-4.2) \times 10^{16} \mathrm{yr}(0 \nu)$ were obtained at the LNGS in the previously described experiment by Belli et al. (2011a).

${ }^{162} \mathrm{Er}$. - The first limit for ${ }^{162} \mathrm{Er}\left(T_{1 / 2}>5.5 \times 10^{8} \mathrm{yr}\right)$ was obtained by Nozzoli (2018) similarly to the limit for ${ }^{144} \mathrm{Sm}$ (valid for all decay modes). Much better results close to $10^{18} \mathrm{yr}$ for specific transitions were obtained in measurements of a highly purified $\mathrm{Er}_{2} \mathrm{O}_{3}$ sample (326 g) with a HPGe detector $\left(465 \mathrm{~cm}^{3}\right)$ for $1934 \mathrm{~h}$ at the LNGS (Belli et al., 2018).

${ }^{164} \mathrm{Er}$.- The first limit of $T_{1 / 2}>1.0 \times 10^{9} \mathrm{yr}$ was obtained by Nozzoli (2018) (valid for all 2EC transitions).

${ }^{168} \mathrm{Yb}$. - The first limit of $T_{1 / 2}>5.7 \times 10^{8} \mathrm{yr}$ was obtained by Nozzoli (2018). A much higher sensitivity for different modes and channels of the decay $\left(\lim T_{1 / 2} \sim 10^{14}-10^{18} \mathrm{yr}\right.$ ) was reached in a low-background experiment with a sample of highly purified ytterbium oxide measured using a lowbackground HPGe detector (Belli et al., 2019b).

${ }^{174} \mathrm{Hf}$.- The first limit $\left(T_{1 / 2}>5.8 \times 10^{8} \mathrm{yr}\right)$ was obtained by Nozzoli (2018). The first counting experiment to search for the $2 \mathrm{EC}$ and $\mathrm{EC} \beta^{+}$decay of ${ }^{174} \mathrm{Hf}$ was realized using a highpurity $180 \mathrm{~g}$ sample of hafnium and the ultralow-background HPGe-detector system located $225 \mathrm{~m}$ underground at the HADES laboratory. After $75 \mathrm{~d}$ of data taking, limits were set at approximately $T_{1 / 2}>10^{16}-10^{18} \mathrm{yr}$ (Danevich et al., 2020).

${ }^{180} \mathrm{~W}$.- - The first experimental limits on the 2EC decays of ${ }^{180} \mathrm{~W}$ were derived in 1995 from measurements with an enriched ${ }^{116} \mathrm{CdWO}_{4}$ crystal scintillator $\left(15 \mathrm{~cm}^{3}\right)$ in the Solotvina Underground Laboratory for $2982 \mathrm{~h}$ (Georgadze et al., 1995). They were upgraded to $\simeq 10^{16} \mathrm{yr}$ with three ${ }^{116} \mathrm{CdWO}_{4}$ crystals (total mass of $330 \mathrm{~g}$ ) and higher statistics (total mass of $14183 \mathrm{~h}$ ), as well as with measurements for $433 \mathrm{~h}$ of a nonenriched $\mathrm{CdWO}_{4}$ crystal $(454 \mathrm{~g})$ (Danevich, Georgadze et al., 1996; Danevich et al., 2003). The limits were further improved in measurements at the LNGS with $\mathrm{ZnWO}_{4}$ scintillators to $\simeq 10^{18}$ yr (Belli et al., 2009a; Belli, Bernabei et al., 2011b), and with $\mathrm{CaWO}_{4}$ scintillating bolometers limits were improved to $\simeq 10^{19} \mathrm{yr}$ (Angloher et al., 2016).

${ }^{184} \mathrm{Os}$. - The first limit on the $\mathrm{EC} \beta^{+}$decay of ${ }^{184} \mathrm{Os}$ $\left(\simeq 10^{10} \mathrm{yr}\right)$ was derived by Tretyak and Zdesenko (1995) from data of the experiment with photoemulsions (Fremlin and Walters, 1952). Searches for the 2EC and $\mathrm{EC} \beta^{+}$decays of ${ }^{184} \mathrm{Os}$ (including possible resonant $0 \nu 2 \mathrm{EC}$ transitions) were performed by Belli et al. (2013a) in measurements at the LNGS with a $173 \mathrm{~g}$ ultrapure Os sample and a $468 \mathrm{~cm}^{3} \mathrm{HPGe}$ detector for $2741 \mathrm{~h}$; this gave limits of $T_{1 / 2} \simeq 10^{16}-10^{17} \mathrm{yr}$.

${ }^{190} \mathrm{Pt}$. - Once again, the first limit on the $\mathrm{EC} \beta^{+}$decay of ${ }^{190} \mathrm{Pt}$ was set from a reanalysis of the photoemulsion experiment (Fremlin and Walters, 1952) by Tretyak and Zdesenko (1995) with $T_{1 / 2} \geq 10^{11} \mathrm{yr}$. $2 \mathrm{EC}$ decays of ${ }^{190} \mathrm{Pt}$ with emission of $\gamma$ quanta were searched for at the LNGS with a $468 \mathrm{~cm}^{3}$ HPGe detector and a $42 \mathrm{~g}$ platinum sample (Belli et al., 2011b) for $1815 \mathrm{~h}$; half-life limits were approximately $10^{16} \mathrm{yr}$.

${ }^{196} \mathrm{Hg}$. - The first experimental limits $\left(\simeq 10^{17} \mathrm{yr}\right)$ on the 2EC in ${ }^{196} \mathrm{Hg}$ were obtained with a $\mathrm{Ge}(\mathrm{Li})$ detector $\left(35 \mathrm{~cm}^{3}\right)$ that was surrounded by an extremely large $(320 \mathrm{~kg}) \mathrm{Hg}$ shield (containing $480 \mathrm{~g}$ of ${ }^{196} \mathrm{Hg}$ ) in the Solotvina Underground Laboratory for $1478 \mathrm{~h}$ (Zdesenko and Kuts, 1986). The sensitivity was further improved by 1 order of magnitude in 1990 with the same $\mathrm{Hg}$ shielding but with a larger HPGe detector $\left(165 \mathrm{~cm}^{3}\right.$ ) over $1109 \mathrm{~h}$ of data taking (Bukhner et al., 1990).

\section{B. Prospects for possible future experiments}

There are currently no large-scale projects to search for $0 \nu 2 \mathrm{EC}$. However, further research (corrections of atomic mass values, discovery of new excited levels) may lead to the identification of nuclides with favorable conditions for the resonant process. In addition, detection of the $0 \nu 2 \mathrm{EC}$ process, along with observation of $0 \nu 2 \beta^{-}$decay in different nuclei [see the discussion by Giuliani, Danevich, and Tretyak (2018)], will be requested if $0 \nu 2 \beta^{-}$decay in one nuclide is registered. Furthermore, the light neutrino exchange mechanism is not unique. The investigations of $\mathrm{EC} \beta^{+}$and $2 \beta^{+}$decay might be one of the tools for identifying the mechanism of the decay, whether it is mediated by light neutrino mass or right-handed current admixture in a weak interaction (Hirsch et al., 1994). Here we examine whether high sensitivity $0 \nu 2 \mathrm{EC}$ experiments are possible in principle.

One can estimate a sensitivity of possible large-scale 2EC experiments assuming the performance of the most advanced $2 \beta^{-}$experiments. For instance, both the GERDA and Majorana ${ }^{76} \mathrm{Ge} 2 \beta$ experiments achieve a background level of $\sim 10^{-3}$ counts $/(\mathrm{yr} \mathrm{keV} \mathrm{kg}$ ) in the region of interest $\sim 2 \mathrm{MeV}$ (Elliott et al., 2017; Agostini et al., 2018). Assuming the use of $100 \mathrm{~kg}$ of a highly radiopure isotopically enriched material, HPGe detectors with a mass of $100 \mathrm{~kg}$, an energy resolution (full width at half maximum) of $3 \mathrm{keV}$, a detection efficiency of $\sim 5 \%$, and a measurement time of $5 \mathrm{yr}$, one can get an estimation of the experimental sensitivity of approximately $\lim T_{1 / 2} \sim(4-9) \times 10^{25} \mathrm{yr}$. Moreover, there are plans for a ton-scale experiment with HPGe detectors Abgrall et al. (2017) that could further improve the sensitivity.

The bolometric detectors, thanks to their excellent energy resolution and the possibility of realizing the calorimetric approach with detectors having different chemical formulas, seem to offer a promising approach in the search for resonant 2EC. Using the level of background estimated by the CUORE Collaboration $\sim 0.004$ counts $/(\mathrm{yr} \mathrm{keV} \mathrm{kg}$ ) (Alessandria et al., 2012), a mass of isotope of $100 \mathrm{~kg}$ [embedded in highly radiopure crystal scintillators (Danevich and Tretyak, 2018)] and a detection efficiency of around $10 \%$ (e.g., in a peak near $Q_{\beta \beta}$ of ${ }^{106} \mathrm{Cd}$ ), one can get an estimation of the experimental sensitivity of approximately $\lim T_{1 / 2} \sim 4 \times 10^{25} \mathrm{yr}$ for a $5 \mathrm{yr}$ experiment.

We emphasize one more advantage of possible large-scale $0 \nu 2 \mathrm{EC}$ experiments over $0 \nu 2 \beta^{-}$searches. In most of the $0 \nu 2 \mathrm{EC}$ processes closest to resonant ones (see Tables XI and XII), the energy of $\gamma$ quanta expected in the decays is many times larger than the energy of the X-ray quanta emitted in the allowed $2 \nu 2 \mathrm{EC}$. For instance, in the case of $0 \nu 2 \mathrm{EC}$ decay of ${ }^{156} \mathrm{Dy}$ to the $0^{+} 1988.5 \mathrm{keV}$ excited level of ${ }^{156} \mathrm{Gd} \gamma$ quanta with energy $1899.5 \mathrm{keV}$ are expected, while energy of the $\mathrm{x}$-ray quanta in the $2 \nu L L$ process in ${ }^{156} \mathrm{Dy}$ should be within 7.2-8.4 keV (the binding energies on the $L_{1}, L_{2}$, and $L_{3}$ shells of gadolinium atom). Thus, the background due to the $2 \nu 2 \mathrm{EC}$ 
mode in an experiment to search for $0 \nu 2 \mathrm{EC}$ decay to excited levels with energy much higher than the energy of x-ray quanta emitted in the $2 \nu 2 \mathrm{EC}$ decay will never play a role in practice in contrast to the $0 \nu 2 \beta^{-}$experiments where background caused by the $2 \nu$ mode becomes dominant due to poor energy resolution (scintillation and some gaseous detectors) (Tretyak and Zdesenko, 1995) or time resolution (lowtemperature bolometers) (Chernyak et al., 2012). However, for the cases of the g.s. to g.s. resonant transitions (e.g., in $\left.{ }^{152} \mathrm{Gd}\right)$, a small energy release $\left.\left[Q_{2 \mathrm{EC}}\left({ }^{152} \mathrm{Gd}\right)=55.7 \mathrm{keV}\right)\right]$ makes separation between the effect searched for and the allowed $2 \nu K L$ process rather problematic practically.

\section{Neutrinoless 2EC with radioactive nuclides}

Since there is no clearly identified "ideal nucleus" to find a resonant 2EC among stable (or long-lived) nuclei, it makes sense to consider experiments with radioactive nuclei. Using a few kilograms of a radioactive material looks challenging but may be possible by taking into account previous experience with the ${ }^{51} \mathrm{Cr}$ source for the GALLEX solar neutrino experiment (Cribier et al., 1996) or the already under way BEST sterile neutrino experiment (Barinov et al., 2019). Possibilities of large amounts of radioactive isotopes production for 2EC experiments are discussed in Sec. VII.C.

The most realistic approach to search for $0 \nu 2 \mathrm{EC}$ decay of radioactive isotopes is $\gamma$ spectrometry. For this purpose one could use a low-background HPGe-detector array and samples of radioactive material as a source. The isotope should be producible in an amount of tens of kilograms, should not cause $\gamma$ background in the region of interest, and the half-life of the isotope relative to the ordinary decay channel should be long enough to carry out several years of measurements and to avoid thermal destruction of the sample. In the most favorable case the main decay channel of the isotope is low-energy $\beta$ (EC) or $\alpha$ decay without $\gamma$-ray emission. The analysis performed by Tretyak et al. (2005) showed that, despite the high energy of the $2 \beta^{-}$decay of some radioactive nuclei (and therefore much faster $0 \nu 2 \beta^{-}$decay), realization of a high sensitivity experiment will be limited mainly by heat release in the detector. However, while the problem is important in the calorimetry approach requested by $2 \beta^{-}$experiments, it becomes less troublesome in the search for $0 \nu 2 \mathrm{EC}$ processes by $\gamma$ spectrometry. Nevertheless, taking into account current rather problematic possibilities for production of long-lived radionuclides in large amounts and experimental challenges in ultralow-background measurements with radioactive samples, this approach is still far from practically realized.

\section{Summary}

To conclude, the highest up-to-date sensitivity to the $0 \nu 2 \mathrm{EC}$ decay of approximately $\lim T_{1 / 2} \sim 10^{21}-10^{22} \mathrm{yr}$ is achieved using gaseous $\left({ }^{78} \mathrm{Kr}\right)$, scintillation $\left({ }^{106} \mathrm{Cd}\right)$, low-temperature bolometric $\left({ }^{40} \mathrm{Ca}\right)$ detectors, $\gamma$ spectrometry with HPGe detectors $\left({ }^{36} \mathrm{Ar},{ }^{58} \mathrm{Ni},{ }^{96} \mathrm{Ru},{ }^{112} \mathrm{Sn}\right)$, and geochemical methods $\left({ }^{130} \mathrm{Ba},{ }^{132} \mathrm{Ba}\right)$. Observation of the allowed two-neutrino mode of 2EC decay is claimed for only three nuclides: ${ }^{78} \mathrm{Kr},{ }^{124} \mathrm{Xe}$, and ${ }^{130} \mathrm{Ba}$. However, the $2 \nu 2 \mathrm{EC}$ processes in ${ }^{78} \mathrm{Kr}$ and ${ }^{124} \mathrm{Xe}$ were detected in a single experiment for each nuclide. Both claims should be confirmed in independent investigations. As for the geochemical result for ${ }^{130} \mathrm{Ba}$, it needs to be approved by detection in direct counting experiments. The sensitivity of the $0 \nu 2 \mathrm{EC}$ experiments is 3 to 4 orders of magnitude lower than that of the most sensitive $0 \nu 2 \beta^{-}$experiments. The main reasons are in general lower sensitivity of the $0 \nu 2 \mathrm{EC}$ experiments to the absolute neutrino mass (with a similar half-life sensitivity), in most cases extremely low isotopic concentration and limited capabilities of the isotopes of interest enrichment, a more complicated $0 \nu 2 \mathrm{EC}$ radiative effect signature (which results in a lower detection efficiency to the $0 \nu 2 \mathrm{EC}$ process), and a typically smaller energy release than those for $0 \nu 2 \beta^{-}$decays, while the smaller energy of the sought-out effect complicates suppression of the radioactive background.

However, the situation might be changed in the case of resonant enhancement of the $0 \nu 2 \mathrm{EC}$. In this case $\gamma$ spectrometry of external isotopically enriched source with the help of HPGe diodes, low-temperature bolometers look the most promising detection techniques for possible large-scale experiments to search for the resonant $0 \nu 2 \mathrm{EC}$ process. In addition to the stable nuclide candidates, searches for the $0 \nu 2 \mathrm{EC}$ process might be considered in radioactive nuclides with a reasonably long half-life and relevant decay mode that does not interfere with the $0 \nu 2 \mathrm{EC}$ effect. The most realistic approach in this case could be $\gamma$ spectrometry with HPGe detectors. However, realization of a high sensitivity experiment with a radioactive isotope is limited by practical difficulties of its production in large amounts.

\section{PRECISE DETERMINATION OF 2EC DECAY ENERGIES}

The probability of $0 \nu 2 \mathrm{EC}$ decay is in general substantially lower than that of $0 \nu 2 \beta$ decay. The only process that can increase the probability of $0 \nu 2 \mathrm{EC}$ decay and thus make it attractive for its experimental search is its resonant enhancement. Assuming that a resonantly enhanced $0 \nu 2 \mathrm{EC}$ transition is found, its use in experiments on the determination of the neutrino type can provide some advantages relative to the investigation of neutrinoless double $\beta^{-}$decay. First, there might be a variety of excited nuclear states with different low spin values and different parities in one nuclide to which the double-electron-capture transition can be resonantly enhanced, resulting in relatively short partial half-lives. Second, no essential reaction induced background from the two-neutrino mode is expected in the case of ground-state-toground-state transitions. For two nuclides connected by a transition between the nuclear ground states only, the neutrinoless mode dominates since the two-neutrino transition is strongly suppressed by phase space: no energy is left for the neutrinos to carry away. Thus, in the past ten years experimenters have focused on the search for nuclides in which such resonantly enhanced $0 \nu 2 \mathrm{EC}$ can take place.

\section{A. Basics of high-precision Penning-trap mass spectrometry}

To determine the degree of resonant enhancement of the $0 \nu 2 \mathrm{EC}$ transitions, their $Q$ values must be measured with an uncertainty of approximately $100 \mathrm{eV}$. The $Q$ value is the 


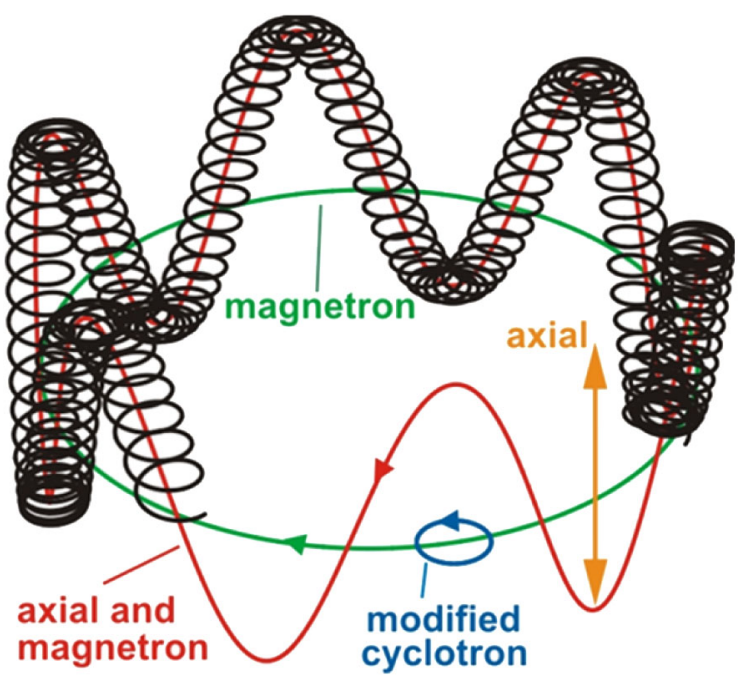

FIG. 15. Motion of an ion in a Penning trap. It can be considered a combination of three independent eigenmotions, cyclotron and magnetron motions in the plane orthogonal to the magnetic-field lines, and axial motion along the magnetic-field line.

mass difference between the initial and final states of the transition

$Q / c^{2}=M_{i}-M_{f}=M_{f}\left(M_{i} / M_{f}-1\right)=M_{f}(R-1)$,

where $M_{i}$ and $M_{f}$ are the atomic masses of the initial and final states, respectively, and $R=M_{i} / M_{f}$ is their ratio. An uncertainty $\delta Q$ of the $Q$-value determination is given by

$$
\delta Q=Q \sqrt{\left(\frac{\delta M_{f}}{M_{f}}\right)^{2}+\left(\frac{\delta R}{R-1}\right)^{2}} \cong Q \frac{\delta R}{R-1}, \quad R>1
$$

where $\delta M_{f}$ and $\delta R$ are the uncertainties of the mass of the final state and the mass ratio, respectively. Thus, a determination of the $Q$ value with an uncertainty of $100 \mathrm{eV}$ implies the measurement of the mass ratio $R$ with an uncertainty of approximately $10^{-9}$.

The only current technique capable of providing such a low uncertainty for a large variety of stable and radioactive nuclides is considered to be high-precision Penning-trap mass spectrometry (PTMS) (Blaum, Dilling, and Nörtershäuser, 2013; Myers, 2013). This technique is superior in achievable sensitivity, accuracy, and resolving power to all other massmeasurement methods due to the very idea that forms the basis of a Penning trap: one confines a single ion with mass $M$ and electrical charge $q$ to a minute volume by the superposition of an extremely stable and strong static homogeneous magnetic field $B$ and weak static quadrupole electric potential. In such a field configuration a charged particle performs a complex periodic motion that is considered to be a product of three circular motions with stable eigenfrequencies, namely, cyclotron, magnetron, and axial motions with the frequencies $\nu_{+}, \nu_{-}$, and $\nu_{z}$, respectively; see Fig. 15 (Brown and Gabrielse, 1986).

A certain combination of these eigenfrequencies yields the so-called free cyclotron frequency

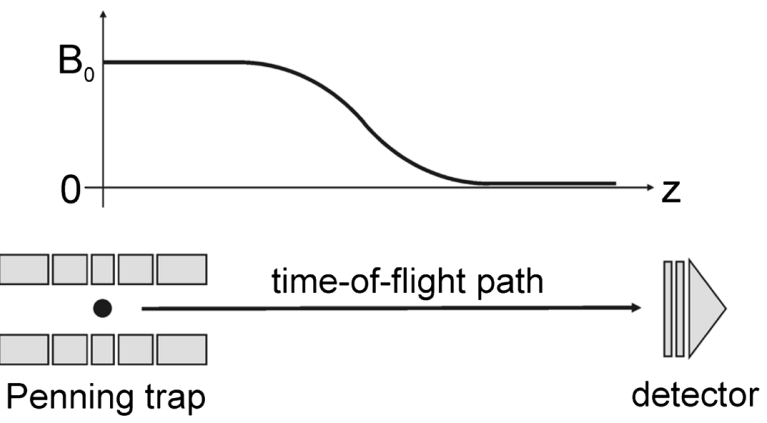

FIG. 16. Basic principle of the TOF-ICR technique. The ions are ejected from the trap and sent to the detector through a strong gradient of the magnetic field. The time of flight of the ions depends on their orbital magnetic moment in the trap and is a function of the ion's cyclotron frequency. For details see the text.

$$
\nu_{c}=\frac{1}{2 \pi} \frac{q}{M} B
$$

i.e., the frequency of an ion with charge-to-mass ratio $q / M$ in a homogeneous magnetic field $B$. A determination of the mass of a charged particle via a measurement of its free cyclotron frequency, the most precisely measurable quantity in physics, is a trick that sets the Penning trap beyond any other massmeasurement technique. The measurement of the mass ratio $R$ reduces to the measurement of the ratio $R=\nu_{c}^{f} / \nu_{c}^{i}$ of the cyclotron frequencies $\nu_{c}^{f}$ and $\nu_{c}^{i}$ of the final and initial states of the transition, respectively.

Most Penning-trap facilities are built for mass measurements on radioactive nuclides and employ for the measurement of the cyclotron frequency the so-called timeof-flight ion-cyclotron-resonance technique (TOF-ICR) (Gräff, Kalinowsky, and Traut, 1980; König et al., 1995). A key component of this method is a microchannel-plate (MCP) detector, which is placed on the axis of the Penning trap (direction $\vec{z}$; see Fig. 17) in a region with low magnetic field. The MCP detector serves as a counter for single ions. The cyclotron frequency is determined from the measurement of the TOF of the ion passing the strong gradient of the magnetic field between the Penning trap and the MCP detector (Fig. 16).

An ion in a magnetic-field gradient is subject to a force that acts in direction $\vec{z}$ and is given by

$$
\vec{F}=-\frac{E_{r}}{B_{0}} \cdot \frac{\partial B}{\partial z} \cdot \vec{z}
$$

where $E_{r}$ is the ion's radial kinetic energy and $B_{0}$ is the magnetic field in the trap. The ion's time of flight between the trap located at zero position and the detector at $z_{\text {det }}$ can thus be determined by

$$
T\left(E_{r}\right)=\int_{0}^{z_{\mathrm{det}}} d z \sqrt{\frac{M}{2\left[E_{0}-q V(z)-\mu\left(E_{r}\right) B(z)\right]}},
$$

where $E_{0}$ is the total initial energy of the ion, $V(z)$ and $B(z)$ are the electric and magnetic fields along the ion's path 


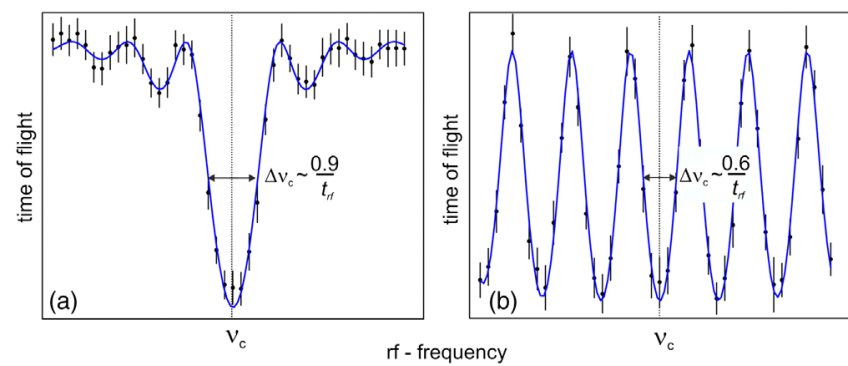

FIG. 17. The line shape of the time of flight vs the (a) one-pulse and (b) two-pulse (Ramsey) rf field of duration $T_{\text {rf }}$, respectively. For details see the text.

between the trap and the MCP detector, and $\mu\left(E_{r}\right)$ is the ion's orbital magnetic moment in the Penning trap.

The orbital magnetic moment of the ion in the trap and hence its time of flight can be manipulated by applying a quadrupolar radio-frequency (rf) field of certain temporal profile at a frequency near the ion's cyclotron frequency in the trap. By varying the frequency of the rf field of duration $T_{\mathrm{rf}}$, one obtains the time of flight versus frequency of the rf field as shown in Fig. 17(a) for a one-pulse rf field (König et al., 1995) and in Fig. 17(b) for a two-pulse rf field (George et al., 2007; George, Blaum et al., 2007; Kretzschmar, 2007).

The two-pulse method (two-pulse Ramsey) is usually the method of choice since it provides the highest precision for the determination of the cyclotron frequency $\nu_{c}$ due to a large number of pronounced periodic minima in the time-of-flight line shape. The cyclotron frequency of singly charged ions of mass $100 \mathrm{u}$ in a magnetic field of $7 \mathrm{~T}$ is approximately $10^{6} \mathrm{~Hz}$. A half-hour measurement of the cyclotron frequency with a rfpulse duration of $2 \mathrm{~s}$ and a two-pulse Ramsey configuration allows for a determination of the cyclotron frequency with a relative uncertainty of about $5 \times 10^{-9}$ to $10^{-8}$. The final achievable uncertainty of the frequency-ratio determination is usually defined by the instability of the magnetic field in time (Droese et al., 2011) and amounts to approximately $10^{-9}$ in experiments that employ the TOF-ICR detection technique.

The novel phase-image ion-cyclotron-resonance (PI-ICR) technique was invented (Eliseev et al., 2013, 2014) for on-line facilities like SHIPTRAP. With this method the measurement of the ion-cyclotron frequency is based on the projection of the ion position in the trap onto a position-sensitive detector. This allows one to monitor the time evolution of the ion motion and thus measure the trap-motion frequencies of the ion with subsequent determination of the ion free cyclotron frequency. Compared to the conventional TOF-ICR technique, the
PI-ICR method offers a gain in precision and resolving power of approximately 5 and 50, respectively. This has made it feasible to carry out measurements of mass ratios of long-lived nuclides with an uncertainty of just a few tens of $\mathrm{eV}$ at on-line Penning-trap facilities (Nesterenko et al., 2014; Eliseev et al., 2015; Karthein et al., 2019).

\section{B. Decay energies of $2 \mathrm{EC}$ transitions in virtually stable nuclides}

Since 12 out of the 19 most promising nuclide pairs from Tables VI and VII have been addressed with SHIPTRAP (Block et al., 2007) and three pairs have been investigated with the mass spectrometers JYFLTRAP (Kolhinen et al., 2004) and TRIGATRAP (Ketelaer et al., 2008) and the Florida State University Penning trap (Shi, Redshaw, and Myers, 2005), which are in many aspects similar to SHIPTRAP, the experiments on the determination of the $Q$ values are described here by the example of the SHIPTRAP mass spectrometer.

The SHIPTRAP facility has been built for experiments on transuranium nuclides produced in fusion-evaporation reactions at GSI, Darmstadt, Germany. A detailed description of the entire facility was given by Block et al. (2007). Here only off-line SHIPTRAP (the part relevant to the measurements of the $Q$ values) is described (Fig. 18).

The nuclides of interest are virtually stable and can be purchased in sufficient amounts in different chemical forms. For production of singly charged ions of these nuclides a laserablation ion source was used (Chaudhuri et al., 2007). For this, a few milligrams of the nuclide of interest were shaped into a $5 \times 5 \mathrm{~mm}^{2}$ solid target on a rotatable holder. These targets were then irradiated with short laser pulses. The frequency-doubled Nd:yttrium-aluminum-garnet laser (532 nm) has a pulse duration of 3-5 ns, a pulse energy of 4-12 mJ, and a diameter of the laser beam on the target of less than $1 \mathrm{~mm}$. The material is ionized by laser induced desorption, fragmentation, and ionization. A series of electrostatic electrodes transport the ions from the ion source toward the Penning-trap mass spectrometer.

The Penning-trap mass spectrometer has two cylindrical Penning traps, the preparation trap (PT) and measurement trap (MT), placed in a magnetic field of $7 \mathrm{~T}$ created by a superconducting magnet. The PT separates the ions of interest from unwanted ions by employing the mass-selective buffer gas cooling technique (Savard et al., 1991). From the PT only the ions of interest pass into the MT, where their cyclotron frequency is measured with the previously described TOF-ICR technique.

TABLE VI. Parameters of the three most promising 2EC transitions between nuclear ground states whose $Q$ values have been precisely measured with SHIPTRAP.

\begin{tabular}{lccc}
\hline \hline & ${ }^{152} \mathrm{Gd} \rightarrow{ }^{152} \mathrm{Sm}$ & ${ }^{164} \mathrm{Er} \rightarrow{ }^{164} \mathrm{Dy}$ & ${ }^{180} \mathrm{~W} \rightarrow{ }^{180} \mathrm{Hf}$ \\
2EC transition & (Eliseev et al., 2011b) & (Eliseev, Novikov, and Blaum, 2012) & (Droese et al., 2012) \\
\hline Electron orbitals & $K L_{1}$ & $L_{1} L_{1}$ & $144.4(45)$ \\
$Q_{2 \mathrm{EC}}($ old) $(\mathrm{keV})$ & $54.6(35)$ & $23.3(39)$ & $12.4(45)$ \\
$\Delta$ (old) (keV) & $0.27(350)$ & $5.05(390)$ & $143.20(27)$ \\
$Q_{2 \mathrm{EC}}($ new $)(\mathrm{keV})$ & $55.70(18)$ & $25.07(12)$ & $11.24(27)$ \\
$\Delta$ (new) $(\mathrm{keV})$ & $0.83(18)$ & $6.82(12)$ & 127 \\
\hline \hline
\end{tabular}


TABLE VII. $2 \mathrm{EC}$ transitions to nuclear excited states that are of interest in the search for the $0 \nu 2 \mathrm{EC}$ process and for which $Q_{2 \mathrm{EC}}$ values have been precisely measured with Penning traps.

\begin{tabular}{|c|c|c|c|c|c|c|}
\hline $0 \nu 2 \mathrm{EC}$ transition & $E^{*}(\mathrm{keV})$ & $J_{f}^{\pi}$ & $\begin{array}{c}\text { Electron } \\
\text { orbitals }\end{array}$ & $Q_{2 \mathrm{EC}}(\mathrm{keV})$ & $\Delta(\mathrm{keV})$ & Reference \\
\hline${ }^{74} \mathrm{Se} \rightarrow{ }^{74} \mathrm{Ge}$ & $1204.205(7)$ & $2^{+}$ & $L_{2} L_{3}$ & $\begin{array}{c}1209.169(49) \\
1209.240(7)\end{array}$ & $\begin{array}{l}2.50(5) \\
2.57(1)\end{array}$ & $\begin{array}{l}\text { Kolhinen et al. (2010) } \\
\text { Mount, Redshaw, and Myers } \\
\quad(2010)\end{array}$ \\
\hline${ }^{78} \mathrm{Kr} \rightarrow{ }^{78} \mathrm{Se}$ & $2838.49(7)$ & $2^{+}$ & $\begin{array}{l}L_{1} M_{4} \\
L_{3} M_{2}\end{array}$ & $2847.75(27)$ & $\begin{array}{l}7.55(28) \\
7.66(28)\end{array}$ & Bustabad et al. (2013) \\
\hline${ }^{96} \mathrm{Ru} \rightarrow{ }^{96} \mathrm{Mo}$ & $2700.21(6)$ & $2^{+}$ & $L_{2} L_{2}$ & 2714.51(13) & $9.05(14)$ & Eliseev, Nesterenko et al. (2011) \\
\hline${ }^{102} \mathrm{Pd} \rightarrow{ }^{102} \mathrm{Ru}$ & $1103.047(13)$ & $2^{+}$ & $K L_{3}$ & $1203.27(36)$ & $75.26(36)$ & Goncharov et al. (2011) \\
\hline${ }^{106} \mathrm{Cd} \rightarrow{ }^{106} \mathrm{Pd}$ & $2748.2(4)$ & $(2,3)^{-}$ & $K L_{3}$ & $2775.39(10)$ & $0.33(41)$ & Goncharov et al. (2011) \\
\hline${ }^{112} \mathrm{Sn} \rightarrow{ }^{112} \mathrm{Cd}$ & $1871.00(19)$ & $0^{+}$ & $K K$ & 1919.82(16) & $4.50(25)$ & Rahaman et al. (2009) \\
\hline${ }^{120} \mathrm{Te} \rightarrow{ }^{120} \mathrm{Sn}$ & & - & & $1714.81(125)$ & & Scielzo et al. (2009) \\
\hline${ }^{124} \mathrm{Xe} \rightarrow{ }^{124} \mathrm{Te}$ & 2790.41(9) & $0^{+}-4^{+}$ & $K K$ & 2856.82(13) & $1.96(16)$ & Nesterenko et al. (2012) \\
\hline${ }^{130} \mathrm{Ba} \rightarrow{ }^{130} \mathrm{Xe}$ & 2544.43(8) & $0^{+}$ & $K K$ & $2623.71(26)$ & $10.15(26)$ & Nesterenko et al. (2012) \\
\hline${ }^{136} \mathrm{Ce} \rightarrow{ }^{136} \mathrm{Ba}$ & $2315.32(7)$ & $0^{+}$ & $K K$ & $2378.53(27)$ & $11.67(28)$ & Kolhinen et al. (2011) \\
\hline${ }^{144} \mathrm{Sm} \rightarrow{ }^{144} \mathrm{Nd}$ & $1560.920(13)$ & $2^{+}$ & $K L_{3}$ & $1782.59(87)$ & $171.89(87)$ & Goncharov et al. (2011) \\
\hline${ }^{156} \mathrm{Dy} \rightarrow{ }^{156} \mathrm{Gd}$ & $\begin{array}{c}1946.375(6) \\
1952.385(7) \\
1988.5(2) \\
2003.749(5)\end{array}$ & $\begin{array}{l}1^{-} \\
0^{-} \\
0^{+} \\
2^{+}\end{array}$ & $\begin{array}{c}K L_{1} \\
K M_{1} \\
L_{1} L_{1} \\
M_{1} N_{3}\end{array}$ & $2005.95(10)$ & $\begin{array}{l}0.75(10) \\
1.37(10) \\
0.54(24) \\
0.04(10)\end{array}$ & Eliseev et al. (2011a) \\
\hline${ }^{162} \mathrm{Er} \rightarrow{ }^{162} \mathrm{Dy}$ & $1782.68(9)$ & $2^{+}$ & $K L_{3}$ & $1846.95(30)$ & $2.69(30)$ & Eliseev, Nesterenko et al. (2011) \\
\hline${ }^{168} \mathrm{Yb} \rightarrow{ }^{168} \mathrm{Er}$ & $1403.7357(23)$ & $2^{-}$ & $M_{2} M_{2}$ & $1409.27(25)$ & $1.52(25)$ & Eliseev, Nesterenko et al. (2011) \\
\hline${ }^{184} \mathrm{Os} \rightarrow{ }^{184} W$ & $1322.152(22)$ & $0^{+}$ & $K K$ & $1453.68(58)$ & $8.89(58)$ & Smorra et al. (2012) \\
\hline${ }^{190} \mathrm{Pt} \rightarrow{ }^{190} \mathrm{Os}$ & $1326.9(5)$ & 1,2 & $\begin{array}{l}K N_{1} \\
K N_{2} \\
K N_{3} \\
K N_{4}\end{array}$ & 1401.57(47) & $\begin{array}{l}0.14(69) \\
0.25(69) \\
0.34(69) \\
0.51(69)\end{array}$ & Eibach et al. (2016) \\
\hline
\end{tabular}

The ratio of the cyclotron frequencies of the initial and final nuclides of the transition is obtained through a measurement of the two cyclotron frequencies alternately, as schematically depicted in Fig. 19 (left panel).

In this case, the ratio that corresponds to measurement time $t_{k}$ [see Fig. 19 (left panel)] is made up of the frequency of the mother nuclide measured at time $t_{k}$ and the frequency of the daughter nuclide that is obtained by linear interpolation of the frequencies of the daughter nuclide measured at times $t_{k-1}$ and $t_{k+1}$. The measurement time of one ratio point usually does not exceed $1 \mathrm{~h}$ and is measured with an uncertainty of better than $10^{-8}$. Thus, a measurement campaign of a few days results in a determination of the frequency ratio with the required uncertainty of about $10^{-9}$, as shown in Fig. 19 (right panel) using the example of double-electron capture in ${ }^{156} \mathrm{Dy}$ (Eliseev et al., 2011a). The corresponding uncertainty of the $Q$ value is about $100 \mathrm{eV}$. The results obtained in this measurement campaign were summarized by Eliseev, Novikov, and Blaum

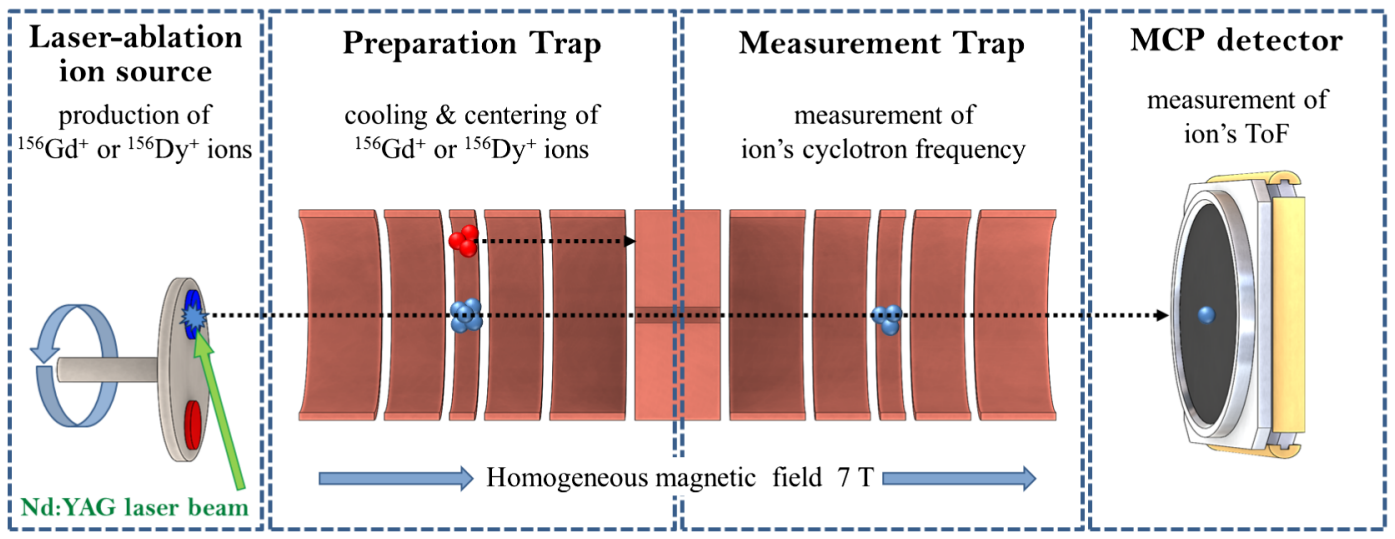

FIG. 18. Sketch of the off-line SHIPTRAP facility. The ions produced with a laser ablation are guided by ion transport optics toward the Penning traps. After the mass-selective cooling and centering of the ions in the preparation trap, they are sent into the measurement trap, where their cyclotron frequency is measured with the TOF-ICR technique. For details see the text. 

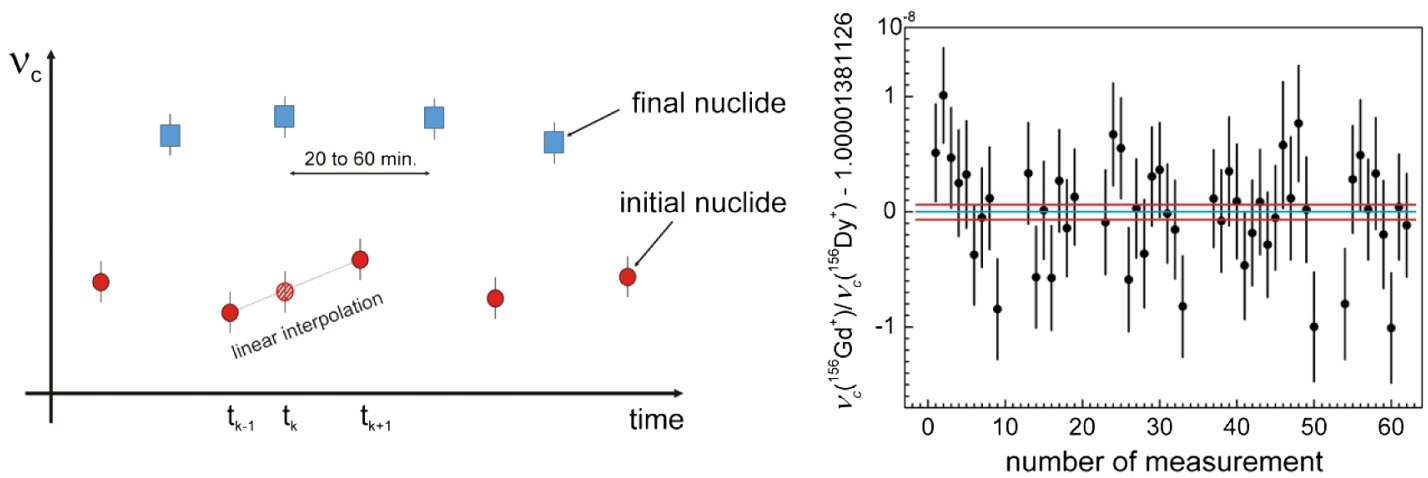

FIG. 19. Illustration of the principle of an alternate measurement of the cyclotron frequencies of two nuclides (left panel) (for details see text), which results in a determination of their ratio (right panel) (using the example of the frequency-ratio measurements of ${ }^{156} \mathrm{Gd}$ and ${ }^{156} \mathrm{Dy}$ ). Left panel: filled squares and filled circles represent the single measurements of the frequencies of the mother and daughter nuclides, respectively. The hatched circle is the value obtained from the linear interpolation to the time $t_{k}$ of the frequencies measured at times $t_{k-1}$ and $t_{k+1}$. Right panel: central and upper and lower lines represent the weighted mean frequency ratio and the error of the determination of the weighted mean frequency ratio, respectively.

(2012) and are included in Tables VI and VII. The choice of nuclide pairs for measurements was made from an analysis of data for all stable nuclides, which from the energy balance can undergo double-electron capture. This dataset is presented in Table V. Presented there, in addition to energy, are other parameters that allow one to judge the proximity of a particular level to the resonant state, leading to an increase in the probability of double capture. These parameters include the energy gap $\Delta$ and the width $\Gamma$ of the states of electrons undergoing capture, on which the resonance gain factor $R$ depends. The most promising cases were selected for the experiments, the results of which are given in Tables VI and VII.

\section{Prospects for measurements of decay energies in radioactive nuclides}

The use of radioactive nuclides in the search for 2EC transitions was first proposed by Berlovich and Novikov (1970). Of particular interest were nuclides that are situated far from the valley of beta stability. They can undergo doubleelectron capture with a much higher decay energy than the nuclides along the valley of beta stability. Since the probability of double-electron capture exhibits a strong dependence on the $Q_{2 \text { EC }}$ value, the half-lives of such nuclides with regard to double-electron capture can be short enough to be measured. As in the case of virtually stable nuclides, here one could search for resonantly enhanced $0 \nu 2 \mathrm{EC}$ by measuring the $Q_{2 \mathrm{EC}}$ values of promising $2 \mathrm{EC}$ transitions.

The main decay mode of such nuclides must not hamper the search for double-electron capture. In other words, it should be either alpha decay to a nuclear ground state or electron capture with a low $Q$ value. Furthermore, from a practical point of view, the half-lives of such nuclides should be at least a few years. The most promising near-resonant nuclides that fulfill these criteria are listed in Table VIII ${ }^{6}$; their specific $0 \nu 2 \mathrm{EC}$ data are given in Table XIII.

\footnotetext{
${ }^{6}$ Center for Nuclear Studies, Department of Physics, The George Washington University. Data Analysis Center: http://www.nndc.bnl .gov/.
}

Provided that a resonantly enhanced transition has been found in these nuclides by means of Penning-trap mass spectrometry, a suitable mechanism will have to be found for a production of macroquantities of these nuclides. Spalation or fragmentation reactions with high-energy particles and a fusion with heavy ions do not allow the production of a quantity that would be sufficient for a large-scale experiment in search of $0 \nu 2 \mathrm{EC}$; see Table IX. ${ }^{7}$ A more promising production mechanism is an irradiation of samples with neutrons in a reactor. Only relatively short-lived nuclides can be produced in this manner; see Table X. To calculate the production yields, the mass of the irradiated sample was taken equal to $1 \mathrm{~mol}$, and the neutron flux was $10^{15} \mathrm{n} /\left(\mathrm{cm}^{2} \mathrm{~s}\right)$. Thus, in principle a production of sufficient amount of ${ }^{169} \mathrm{Yb}$ and ${ }^{175} \mathrm{Hf}$ is feasible. However, since these nuclides are too short lived, their handling might be problematic.

\section{NORMALIZED HALF-LIVES OF NEAR-RESONANT NUCLIDES}

In this section, estimates of the $0 \nu 2 \mathrm{EC}$ half-lives of nuclei closest to the resonance condition are presented. Over the past ten years, great progress has been made due to accurate measurements of $Q_{2 \mathrm{EC}}$ values, which made it possible to clarify whether the resonance condition for the prospective nuclides is satisfied; see Sec. VII. The $Q_{2 \mathrm{EC}}$ values of the identified earlier prospective nuclides have now been measured.

\section{A. Decays of virtually stable nuclides}

New estimates of the half-lives, taking the recent $Q$-value measurements into account, are presented in Tables XI and XII. In view of the considerable variance of the NME values for various nuclides (Table IV) and to not mix distinct physical effects, the normalized half-lives $\tilde{T}_{1 / 2}$ with NME $=3$ are provided. In the following we focus mainly on the light Marjorana neutrino exchange mechanism of Fig. 3(a).

Astrophysical restrictions on the sum of the diagonal neutrino masses obtained by the Planck Collaboration from

\footnotetext{
${ }^{7}$ See http://isolde.web.cern.ch.
} 
TABLE VIII. Radioactive nuclides with half-lives $T_{1 / 2}>1 \mathrm{yr}$, which might be of interest for $0 \nu 2 \mathrm{EC}$. $Q_{\alpha}$ and $T_{1 / 2}^{\alpha}$ are the $Q$ value and half-life of the $\alpha$-decay mode, respectively. The $Q_{2 \mathrm{EC}}, Q_{\mathrm{EC}}$, and $Q_{\alpha}$ values and $T_{1 / 2}^{\mathrm{EC}}$ are from Wang et al. (2017), and $T_{1 / 2}$ are from the database of Brookhaven National Laboratory.

\begin{tabular}{lccccccc}
\hline \hline $\begin{array}{l}\text { Mother } \\
\text { nuclide }\end{array}$ & $\begin{array}{c}T_{1 / 2} \\
(\mathrm{yr})\end{array}$ & $\begin{array}{c}Q_{2 \mathrm{EC}} \\
(\mathrm{keV})\end{array}$ & $\begin{array}{c}T_{1 / 2}^{2 \mathrm{ECa}} \\
(\mathrm{yr})\end{array}$ & $\begin{array}{c}Q_{\mathrm{EC}} \\
(\mathrm{keV})\end{array}$ & $\begin{array}{c}T_{1 / 2}^{\mathrm{EC}} \\
(\mathrm{yr})\end{array}$ & $\begin{array}{c}Q_{\alpha} \\
(\mathrm{keV})\end{array}$ & $\begin{array}{c}T_{1 / 2}^{\alpha} \\
(\mathrm{yr})\end{array}$ \\
\hline${ }^{148} \mathrm{Gd}$ & $71.1 \pm 1.2$ & $3066.9(9)$ & $10^{25-33}$ & $30(10)$ & $10^{3}$ & 3271 & 70 \\
${ }^{150} \mathrm{Gd}$ & $(1.79 \pm 0.08) \times 10^{6}$ & $1287(6)$ & $10^{23-33}$ & $\ldots$ & $\ldots$ & 2807 & $(3.00 \pm 0.15) \times 10^{6}$ \\
${ }^{154} \mathrm{Dy}$ & $(3.0 \pm 1.5) \times 10^{6}$ & $3312(7)$ & $10^{23-30}$ & $\ldots$ & $\ldots$ & $3 \times 10^{6}$ \\
${ }^{194} \mathrm{Hg}$ & $444 \pm 77$ & $2576(3)$ & $10^{25-31}$ & $28(4)$ & $10^{3}$ & 2698 & $\sim 10^{20 \mathrm{a}}$ \\
${ }^{202} \mathrm{~Pb}$ & $(5.25 \pm 0.28) \times 10^{4}$ & $1405(4)$ & $10^{22-32}$ & $40(4)$ & $10^{2}$ & 2589 & $\sim 10^{23 \mathrm{~b}}$ \\
\hline \hline
\end{tabular}

${ }^{\mathrm{a}}$ According to Sec. VIII.

${ }^{b}$ Values for alpha decay estimated with the Geiger-Nuttal approach (Brown, 1992).

TABLE IX. Estimated yields for the production of the radioactive nuclides of interest for the search for neutrinoless $2 \mathrm{EC}$ in spalation or fragmentation reactions with high-energy particles and fusion with heavy ions.

\begin{tabular}{lcccc}
\hline \hline $\begin{array}{l}\text { Mother } \\
\text { nuclide }\end{array}$ & $\begin{array}{c}\text { ISOLDE/CERN } \\
\text { counts/ions } / \mu \mathrm{C}\end{array}$ & $\begin{array}{c}\text { yield/atoms } \\
\text { (spallation, } 3 \text { months) }\end{array}$ & $\begin{array}{c}\text { yield/atoms } \\
\left.\text { (fusion with }{ }^{48} \mathrm{Ca}, 3 \mathrm{months}\right)\end{array}$ & $\begin{array}{c}\text { Max. counts } \\
|\lambda Y|_{2 \mathrm{EC}} / \mathrm{yr}^{-1}\end{array}$ \\
\hline${ }^{148} \mathrm{Gd}$ & $6 \times 10^{8}$ & $\approx 10^{16}$ & Too small \\
${ }^{150} \mathrm{Gd}$ & $4 \times 10^{8}$ & $\approx 10^{16}$ & $\approx 10^{13}$ & $\approx 10^{-7}$ \\
${ }^{154} \mathrm{Dy}$ & $8 \times 10^{9}$ & $\approx 10^{17}$ & $\approx 10^{13}$ & $\approx 10^{-6}$ \\
${ }^{194} \mathrm{Hg}$ & $7 \times 10^{9}$ & $\approx 10^{17}$ & $\approx 10^{13}$ & Too small \\
${ }^{202} \mathrm{~Pb}$ & $10^{7}$ & $\approx 10^{14}$ & $\approx 10^{-9}$ \\
\hline \hline
\end{tabular}

TABLE X. Parameters of relatively short-lived nuclides with near-resonant transitions for the search for $0 \nu 2 \mathrm{EC}$, which can be produced in a reactor. The $Q_{\mathrm{EC}}$ and $Q_{2 \mathrm{EC}}$ values are in $\mathrm{keV}$ units (Wang et al., 2017), $E^{*}$ are in $\mathrm{keV}$ units, and $J_{i}^{\pi}$ are from the database of the Brookhaven National Laboratory.

\begin{tabular}{|c|c|c|c|c|c|c|}
\hline Nuclide & $J_{i}^{\pi}$ & $T_{1 / 2}^{\exp }$ & $Q_{\mathrm{EC}}$ & $Q_{2 \mathrm{EC}}$ & $\begin{array}{l}E^{*}, \text { possible relevant levels } \\
\text { in daughter nuclide }\end{array}$ & $\begin{array}{c}|\lambda Y|_{\mathrm{EC}} / \mathrm{y}^{-1} \\
(3 \text { months in reactor) }\end{array}$ \\
\hline$\overline{93} \mathrm{Mo}$ & $5 / 2^{+}$ & $(4.0 \pm 0.8) \times 10^{3} \mathrm{yr}$ & $405.8(15)$ & $315.0(5)$ & $266.8\left(3 / 2^{+}\right)$ & $10^{-3}$ \\
\hline${ }^{113} \mathrm{Sn}$ & $1 / 2^{+}$ & $115.09 \pm 0.03 \mathrm{~d}$ & $1039.0(16)$ & $715.2(16)$ & $680.52\left(3 / 2^{+}\right), 708.57\left(5 / 2^{+}\right)$ & $3 \times 10^{-3}$ \\
\hline${ }^{145} \mathrm{Sm}$ & $7 / 2^{-}$ & $340 \pm 3 d$ & $616.2(25)$ & $780.6(9)$ & $748.275\left(9 / 2^{-}\right)$ & $10^{-2}$ \\
\hline${ }^{169} \mathrm{Yb}$ & $7 / 2^{-}$ & $32.018 \pm 0.005 \mathrm{~d}$ & $897.6(11)$ & $545.5(3)$ & & 10 \\
\hline${ }^{175} \mathrm{Hf}$ & $5 / 2^{(-)}$ & $70 \pm 2 \mathrm{~d}$ & $683.9(20)$ & $213.9(23)$ & & 4 \\
\hline
\end{tabular}

TABLE XI. The $0 \nu 2 \mathrm{EC}$ processes closest to the resonant ones. The normalized half-lives $\tilde{T}_{1 / 2}$ take newly measured $Q_{2 \mathrm{EC}}$ values into account. The first column reports the natural isotopic abundance $(l)$ of the parent nuclides. The spin and parity of the daughter nuclides are given in column 3. If the spin or parity are unknown, their suggested or assumed values are given in round or square brackets, respectively. Column 4 reports the excitation energies of daughter nuclides together with the experimental errors. Column 5 lists the degeneracy parameter of the two atoms $\Delta=M_{A, Z-2}^{* *}-M_{A, Z}$, including the excitation energy of the electron shell; the errors indicate the experimental uncertainty in the $Q_{2 \mathrm{EC}}$ values. The quantum numbers of the electron vacancies $\alpha$ and $\beta$ are given in the next two columns, where $n$ is the principal quantum number, $j$ is the total angular momentum, and $l$ is the orbital angular momentum. Columns 8, 9, and 10 enumerate the energies of the vacancies $\epsilon_{\alpha}^{*}$ and $\epsilon_{\beta}^{*}$ and the energy shift $\Delta \epsilon_{\alpha \beta}^{*}$ due to the Coulomb interaction, relativistic, and collective electron shell effects. Column 11 presents the widths of the excited electron shells. The minimum and maximum normalized half-lives (in years) corresponding to the $99 \%$ C.L. interval determined by the uncertainty in the degeneracy parameter $\Delta$ are presented in the last two columns. The masses, energies, and widths are given in keV.

\begin{tabular}{|c|c|c|c|c|c|c|c|c|c|c|c|c|}
\hline$l$ & Tra & $J_{f}^{\pi}$ & $M_{A, Z-2}^{*}-M_{A, Z-2}$ & $M_{A, Z-2}^{* *}-M_{A, Z}$ & $(n 2 j l)_{\alpha}$ & $(n 2 j l)_{\beta}$ & $\epsilon_{\alpha}^{*}$ & $\epsilon_{\beta}^{*}$ & $\Delta \epsilon_{\alpha \beta}^{*}$ & $\Gamma_{\alpha \beta}$ & $\tilde{T}_{1 / 2}^{\min }$ & $\tilde{T}_{1 / 2}^{\max }$ \\
\hline $52 \%$ & ${ }_{44}^{96} \mathrm{Ru} \rightarrow{ }_{42}^{96} \mathrm{Mo}^{* *}$ & 0 & $2742 \pm 1$ & $28.1 \pm 0.13$ & 310 & 410 & 0.50 & 0.06 & 0.02 & $9.5 \times 10^{-3}$ & $1.0 \times 10^{37}$ & $1.3 \times 10^{37}$ \\
\hline $1.25 \%$ & ${ }_{48}^{106} \mathrm{Cd} \rightarrow{ }_{46}^{106} \mathrm{Pd}^{* *}$ & {$\left[0^{+}\right]$} & & $\begin{array}{r}11.0 \pm 0.10 \\
-10.3 \pm 0.10\end{array}$ & $\begin{array}{l}110 \\
110\end{array}$ & $\begin{array}{l}110 \\
210\end{array}$ & $\begin{array}{l}24.35 \\
24.35\end{array}$ & $\begin{array}{r}24.35 \\
3.60\end{array}$ & $\begin{array}{l}0.72 \\
0.18\end{array}$ & $\begin{array}{l}1.3 \times 10^{-2} \\
1.0 \times 10^{-2}\end{array}$ & $\begin{array}{l}8 \times 10^{31} \\
3 \times 10^{32}\end{array}$ & $\begin{array}{l}2 \times 10^{32} \\
8 \times 10^{32}\end{array}$ \\
\hline $0.095 \%$ & ${ }_{54}^{124} \mathrm{Xe} \rightarrow{ }_{52}^{124} \mathrm{Te}^{* *}$ & {$\left[0^{+}\right]$} & $2853.2 \pm 0.6$ & $\begin{array}{l}2.5 \pm 0.12 \\
1.6 \pm 0.12\end{array}$ & $\begin{array}{l}210 \\
210\end{array}$ & $\begin{array}{l}310 \\
410\end{array}$ & $\begin{array}{l}4.94 \\
4.94\end{array}$ & $\begin{array}{l}1.01 \\
0.17\end{array}$ & $\begin{array}{l}0.06 \\
0.02\end{array}$ & $\begin{array}{l}1.2 \times 10^{-2} \\
4.6 \times 10^{-3}\end{array}$ & $\begin{array}{l}5 \times 10^{31} \\
2 \times 10^{29}\end{array}$ & $\begin{array}{l}1 \times 10^{33} \\
3 \times 10^{35}\end{array}$ \\
\hline $185 \%$ & ${ }_{58}^{136} \mathrm{Ce} \rightarrow{ }_{56}^{136} \mathrm{Ba}^{* *}$ & $\begin{array}{c}0^{+} \\
{\left[0^{+}\right]}\end{array}$ & $\begin{array}{c}2315.32 \pm 0.07^{\mathrm{a}} \\
2349.5 \pm 0.5\end{array}$ & $\begin{array}{l}12.6 \pm 0.27 \\
46.7 \pm 0.27 \\
14.6 \pm 0.27\end{array}$ & $\begin{array}{l}110 \\
110 \\
110\end{array}$ & $\begin{array}{l}110 \\
110 \\
210\end{array}$ & $\begin{array}{l}37.44 \\
37.44 \\
37.44\end{array}$ & $\begin{array}{r}37.44 \\
37.44 \\
5.99\end{array}$ & $\begin{array}{l}0.93 \\
0.93 \\
0.23\end{array}$ & $\begin{array}{l}2.6 \times 10^{-2} \\
2.6 \times 10^{-2} \\
1.5 \times 10^{-2}\end{array}$ & $\begin{array}{l}2 \times 10^{31} \\
2 \times 10^{32} \\
1 \times 10^{32}\end{array}$ & $\begin{array}{l}2 \times 10^{31} \\
2 \times 10^{32} \\
2 \times 10^{32}\end{array}$ \\
\hline
\end{tabular}


TABLE XI. (Continued)

\begin{tabular}{|c|c|c|c|c|c|c|c|c|c|c|c|c|}
\hline$l$ & Tran & $J_{f}^{\pi}$ & $M_{A, Z-2}^{*}-M_{A, Z-2}$ & $M_{A, Z-2}^{* *}-M_{A, Z}$ & $(n 2 j l)_{\alpha}$ & $(n 2 j l)_{\beta}$ & $\epsilon_{\alpha}^{*}$ & $\epsilon_{\beta}^{*}$ & $\Delta \epsilon_{\alpha \beta}^{*}$ & $\Gamma_{\alpha \beta}$ & $\tilde{T}_{1 / 2}^{\min }$ & $\tilde{T}_{1 / 2}^{\max }$ \\
\hline & & & & $9.7 \pm 0.27$ & 110 & 310 & 37.44 & 1.29 & 0.08 & $2.4 \times 10^{-2}$ & $2 \times 10^{32}$ & $3 \times 10^{32}$ \\
\hline & & $\left(1^{+}, 2^{+}\right)$ & $2392.1 \pm 0.6$ & $20.9 \pm 0.27$ & 210 & 310 & 5.99 & 1.29 & 0.05 & $1.3 \times 10^{-2}$ & $3 \times 10^{33}$ & $5 \times 10^{33}$ \\
\hline & & $\left(1^{+}, 2^{+}\right)$ & $2399.87 \pm 0.05$ & $64.9 \pm 0.27$ & 110 & 210 & 37.44 & 5.99 & 0.19 & $1.5 \times 10^{-2}$ & $1 \times 10^{33}$ & $1 \times 10^{33}$ \\
\hline \multirow[t]{5}{*}{$0.20 \%$} & ${ }_{64}^{152} \mathrm{Gd} \rightarrow{ }_{62}^{152} \mathrm{Sm}^{* *}$ & $0^{+}$ & 0 & $39.0 \pm 0.18$ & 110 & 110 & 46.83 & 46.83 & 1.09 & $4.0 \times 10^{-2}$ & $5 \times 10^{31}$ & $5 \times 10^{31}$ \\
\hline & & & & $-0.9 \pm 0.18$ & 110 & 210 & 46.83 & 7.74 & 0.28 & $2.3 \times 10^{-2}$ & $3 \times 10^{28}$ & $3 \times 10^{29}$ \\
\hline & & & & $-1.3 \pm 0.18$ & 110 & 211 & 46.83 & 7.31 & 0.33 & $2.3 \times 10^{-2}$ & $1 \times 10^{31}$ & $5 \times 10^{31}$ \\
\hline & & & & $-7.1 \pm 0.18$ & 110 & 310 & 46.83 & 1.72 & 0.09 & $3.2 \times 10^{-2}$ & $3 \times 10^{31}$ & $3 \times 10^{31}$ \\
\hline & & & & $-8.5 \pm 0.18$ & 110 & 410 & 46.83 & 0.35 & 0.04 & $2.4 \times 10^{-2}$ & $2 \times 10^{32}$ & $3 \times 10^{32}$ \\
\hline \multirow[t]{17}{*}{$0.06 \%$} & ${ }_{66}^{156} \mathrm{Dy} \rightarrow{ }_{64}^{156} \mathrm{Gd}^{* *}$ & $1^{-}$ & $1946.375 \pm 0.006$ & $-1.1 \pm 0.10$ & 110 & 211 & 50.24 & 7.93 & 0.33 & $2.6 \times 10^{-2}$ & $5 \times 10^{29}$ & $2 \times 10^{30}$ \\
\hline & & & & $-7.6 \pm 0.10$ & 110 & 311 & 50.24 & 1.69 & 0.10 & $2.8 \times 10^{-2}$ & $2 \times 10^{32}$ & $2 \times 10^{32}$ \\
\hline & & $0^{-}$ & $1952.385 \pm 0.007$ & $5.3 \pm 0.10$ & 110 & 210 & 50.24 & 8.38 & 0.29 & $2.6 \times 10^{-2}$ & $8 \times 10^{31}$ & $1 \times 10^{32}$ \\
\hline & & & & $-1.4 \pm 0.10$ & 110 & 310 & 50.24 & 1.88 & 0.10 & $3.5 \times 10^{-2}$ & $1 \times 10^{31}$ & $3 \times 10^{31}$ \\
\hline & & $1^{-}$ & $1962.037 \pm 0.012$ & $14.5 \pm 0.10$ & 110 & 211 & 50.24 & 7.93 & 0.33 & $2.6 \times 10^{-2}$ & $2 \times 10^{32}$ & $2 \times 10^{32}$ \\
\hline & & & & $8.1 \pm$ & 110 & 311 & 50.24 & 1.69 & 0.10 & $2.8 \times 10^{-2}$ & $2 \times 10^{32}$ & $2 \times 10^{32}$ \\
\hline & & $1^{+}$ & $1965.950 \pm 0.004$ & $18.8 \pm 0.10$ & 110 & 210 & 50.24 & 8.38 & 0.23 & $2.6 \times 10^{-2}$ & $1 \times 10^{31}$ & $1 \times 10^{31}$ \\
\hline & & & & $12.2 \pm 0.10$ & 110 & 310 & 50.24 & 1.88 & 0.08 & $3.5 \times 10^{-2}$ & $2 \times 10^{31}$ & $2 \times 10^{31}$ \\
\hline & & & & $10.6 \pm 0.10$ & 110 & 410 & 50.24 & 0.38 & 0.03 & $2.7 \times 10^{-2}$ & $8 \times 10^{31}$ & $8 \times 10^{31}$ \\
\hline & & {$\left[0^{+}\right]$} & $1970.2 \pm 0.8$ & $65.8 \pm 0.10$ & 110 & 110 & 50.24 & 50.24 & 1.14 & $4.5 \times 10^{-2}$ & $8 \times 10^{31}$ & $8 \times 10^{31}$ \\
\hline & & & & $23.1 \pm 0.10$ & 110 & 210 & 50.24 & 8.38 & 0.29 & $2.6 \times 10^{-2}$ & $5 \times 10^{31}$ & $8 \times 10^{31}$ \\
\hline & & & & $16.4 \pm 0.10$ & 110 & 310 & 50.24 & 1.88 & 0.10 & $3.5 \times 10^{-2}$ & $8 \times 10^{31}$ & $1 \times 10^{32}$ \\
\hline & & $0^{+}$ & $1988.5 \pm 0.2^{\mathrm{a}}$ & $84.1 \pm 0.10$ & 110 & 110 & 50.24 & 50.24 & 1.14 & $4.5 \times 10^{-2}$ & $1 \times 10^{32}$ & $1 \times 10^{32}$ \\
\hline & & & & $41.4 \pm 0.10$ & 110 & 210 & 50.24 & 8.38 & 0.29 & $2.6 \times 10^{-2}$ & $2 \times 10^{32}$ & $2 \times 10^{32}$ \\
\hline & & & & $-0.6 \pm 0.10$ & 210 & 210 & 8.38 & 8.38 & 0.19 & $7.6 \times 10^{-3}$ & $8 \times 10^{25}$ & $8 \times 10^{30}$ \\
\hline & & & & $-1.1 \pm 0.10$ & 210 & 211 & 8.38 & 7.93 & 0.14 & $7.7 \times 10^{-3}$ & $8 \times 10^{31}$ & $8 \times 10^{32}$ \\
\hline & & $0^{+}$ & $2026.664 \pm 0.006$ & $79.5 \pm 0.10$ & 110 & 210 & 50.24 & 8.38 & 0.23 & $2.6 \times 10^{-2}$ & $2 \times 10^{32}$ & $2 \times 10^{32}$ \\
\hline \multirow[t]{4}{*}{$0.14 \%$} & ${ }_{70}^{168} \mathrm{Yb}-$ & $1^{-}$ & $1358.898 \pm 0.005$ & $16.7 \pm 0.25$ & 110 & 211 & 57.49 & 9.26 & 0.36 & $3.3 \times 10^{-2}$ & $8 \times 10^{31}$ & $1 \times 10^{32}$ \\
\hline & & & & $9.2 \pm 0.25$ & 110 & 311 & 57.49 & 2.01 & 0.11 & $3.5 \times 10^{-2}$ & $8 \times 10^{31}$ & $1 \times 10^{32}$ \\
\hline & & & & $7.5 \pm 0.25$ & 110 & 411 & 57.49 & 0.37 & 0.04 & $3.4 \times 10^{-2}$ & $2 \times 10^{32}$ & $3 \times 10^{32}$ \\
\hline & & $0^{+}$ & $1422.10 \pm 0.03$ & $129.0 \pm 0.25$ & 110 & 110 & 57.49 & 57.49 & 1.25 & $5.7 \times 10^{-2}$ & $1 \times 10^{32}$ & $2 \times 10^{32}$ \\
\hline \multirow[t]{2}{*}{$0.13 \%$} & ${ }_{74}^{180} \mathrm{~W} \rightarrow{ }_{72}^{180} \mathrm{Hf}^{*}$ & $0^{+}$ & 0 & $-11.2 \pm 0.27$ & 110 & 110 & 65.35 & 65.35 & 1.36 & $7.2 \times 10^{-2}$ & $5 \times 10^{29}$ & $5 \times 10^{29}$ \\
\hline & & & & $-66.2 \pm 0.27$ & 110 & 210 & 65.35 & 11.27 & 0.36 & $4.2 \times 10^{-2}$ & $1 \times 10^{32}$ & $1 \times 10^{32}$ \\
\hline \multirow[t]{2}{*}{$0.02 \%$} & ${ }_{76}^{184} \mathrm{Os} \rightarrow{ }_{74}^{184} \mathrm{~W}^{* *}$ & $(0)^{+}$ & $1322.152 \pm 0.022^{\mathrm{a}}$ & $8.8 \pm 0.58$ & 110 & 110 & 69.53 & 69.53 & 1.42 & $8.0 \times 10^{-2}$ & $2 \times 10^{29}$ & $3 \times 10^{29}$ \\
\hline & & & & $-49.6 \pm 0.58$ & 110 & 210 & 69.53 & 12.10 & 0.37 & $4.6 \times 10^{-2}$ & $3 \times 10^{31}$ & $5 \times 10^{31}$ \\
\hline \multirow[t]{4}{*}{$0.014 \%$} & ${ }_{78}^{190} \mathrm{Pt} \rightarrow{ }_{76}^{190} \mathrm{Os}^{* *}$ & $(0,1,2)^{+}$ & $1382.4 \pm 0.2^{\mathrm{a}}$ & $7.0 \pm 0.47$ & 210 & 210 & 12.97 & 12.97 & 0.24 & $1.4 \times 10^{-2}$ & $2 \times 10^{31}$ & $33 \times 10^{31}$ \\
\hline & & & & $-3.0 \pm 0.47$ & 210 & 310 & 12.97 & 3.05 & 0.11 & $2.2 \times 10^{-2}$ & $2 \times 10^{30}$ & $1 \times 10^{31}$ \\
\hline & & & & $-3.3 \pm 0.47$ & 210 & 311 & 12.97 & 2.79 & 0.09 & $1.6 \times 10^{-2}$ & $2 \times 10^{32}$ & $1 \times 10^{33}$ \\
\hline & & & & $-5.5 \pm 0.47$ & 210 & 410 & 12.97 & 0.65 & 0.05 & $1.5 \times 10^{-2}$ & $8 \times 10^{31}$ & $2 \times 10^{32}$ \\
\hline
\end{tabular}

${ }^{\mathrm{a}}$ Decay channels with the known NMEs listed in Table IV.

the study of cosmic microwave background anisotropies yield $\sum m_{\nu}<120 \mathrm{meV}$ (Aghanim et al., 2018). The best restrictions on the effective electron neutrino Majorana mass in double-beta-decay experiments were obtained by the KamLAND-Zen Collaboration (Gando et al., 2016): $\left|m_{\beta \beta}\right|<61-165 \mathrm{meV}$ for commonly used NMEs and the unquenched axial-vector coupling $g_{A}=1.27$. Exotic scalar interactions modify the mass of neutrinos in nuclear matter (Kovalenko, Krivoruchenko, and Šimkovic, 2014), so the effective electron neutrino Majorana mass in $0 \nu 2 \beta^{-}$decay and $0 \nu 2 \mathrm{EC}$ decay can differ from that derived from astrophysical data and tritium beta decay.

The decay half-life is determined by the decay width: $T_{1 / 2}^{0 \nu 2 \mathrm{EC}}=\ln 2 / \Gamma_{i}$, where $\Gamma_{i}$ is given by Eq. (3.6), with $M_{i}=$ $M_{A, Z}$ and $M_{f}=M_{A, Z-2}^{* *} ; \Gamma_{f} \equiv \Gamma_{\alpha \beta}$ is the decay width of the daughter atom with vacancies of electrons in the states $\alpha$ and $\beta$. The amplitude $V_{\alpha \beta}$ entering $\Gamma_{i}$ is defined by Eq. (2.14); for the light Majorana neutrino exchange mechanism, $V_{\alpha \beta}$ simplifies to Eq. (3.21).

Assuming that the light Majorana neutrino exchange mechanism is dominant, $T_{1 / 2}^{0 \nu 2 \mathrm{EC}}$ scales with powers of the overlap factor $K_{Z}$, the neutrino mass $m_{\beta \beta}$, the axial-vector coupling $g_{A}$, and the nuclear matrix element $M_{2 \mathrm{EC}}$. The decay half-life can be written as follows:

$T_{1 / 2}^{0 \nu 2 \mathrm{EC}}=K_{Z}^{-2}\left(\frac{1.27}{g_{A}}\right)^{4}\left(\frac{100 \mathrm{meV}}{\left|m_{\beta \beta}\right|}\right)^{2}\left(\frac{3}{\left|M_{2 \mathrm{EC}}\right|}\right)^{2} \tilde{T}_{1 / 2}$.

The normalized half-life $\tilde{T}_{1 / 2}$ does not depend on $K_{Z}, m_{\beta \beta}, g_{A}$, or $M^{2 \mathrm{EC}}$. Various schemes for calculating $K_{Z}$ were discussed 
TABLE XII. The normalized half-lives of $0 \nu 2 \mathrm{EC}$ processes not included in Table XI, but for which precise $Q_{2 \mathrm{EC}}$ values are known and/or experimental constraints on the $0 \nu 2 \mathrm{EC}$-decay half-lives exist. Transitions with $\tilde{T}_{1 / 2}^{\min }>10^{35} \mathrm{yr}$ are not shown. Other notations are the same as in Table XI

\begin{tabular}{|c|c|c|c|c|c|c|c|c|c|c|c|c|}
\hline$l$ & on & $J_{f}^{\pi}$ & $M_{A, Z-2}^{*}-M_{A, Z-2}$ & $M_{A, Z-2}^{* *}-M_{A, Z}$ & $(n 2 j l)_{\alpha}$ & $(n 2 j l)_{\beta}$ & $\epsilon_{\alpha}^{*}$ & $\epsilon_{\beta}^{*}$ & $\Delta \epsilon_{\alpha \beta}^{*}$ & $\Gamma_{\alpha \beta}$ & $\tilde{T}_{1 / 2}^{\min }$ & $\tilde{T}_{1 / 2}^{\max }$ \\
\hline \multirow[t]{2}{*}{$5.52 \%$} & ${ }_{44}^{96} \mathrm{Ru} \rightarrow{ }_{42}^{96} \mathrm{Mo}^{* *}$ & {$\left[0^{+}\right]$} & $2712.68 \pm 0.10^{\mathrm{a}}$ & $1.6 \pm 0.13$ & 210 & 310 & 2.87 & 0.50 & 0.05 & $1.0 \times 10^{-2}$ & $5 \times 10^{32}$ & $2 \times 10^{33}$ \\
\hline & & $0^{+}$ & $2742 \pm 1$ & $68.1 \pm 0.13$ & 110 & 110 & 20.00 & 20.00 & 0.64 & $9.0 \times 10^{-3}$ & $1 \times 10^{34}$ & $1 \times 10^{34}$ \\
\hline \multirow[t]{2}{*}{$1.25 \%$} & ${ }_{48}^{106} \mathrm{Cd} \rightarrow{ }_{46}^{106} \mathrm{Pd}^{* *}$ & {$\left[0^{+}\right]$} & $2717.59 \pm 0.21^{\mathrm{a}}$ & $-8.4 \pm 0.10$ & 110 & 110 & 24.35 & 24.35 & 0.72 & $1.3 \times 10^{-2}$ & $5 \times 10^{31}$ & $8 \times 10^{31}$ \\
\hline & & $2,3^{-}$ & $2748.2 \pm 0.4$ & $0.5=$ & 110 & 231 & 24.35 & 3.17 & 0.18 & $8.3 \times 10^{-3}$ & $2 \times 10^{31}$ & $2 \times 10^{36}$ \\
\hline $.97 \%$ & ${ }_{50}^{112} \mathrm{Sn} \rightarrow{ }_{48}^{112} \mathrm{Cd}^{* *}$ & $0^{+}$ & $1871.00 \pm 0.19$ & $5.4 \pm 0.16$ & 110 & 110 & 26.71 & 26.71 & 0.76 & $1.5 \times 10^{-2}$ & $1 \times 10^{31}$ & $2 \times 10^{31}$ \\
\hline \multirow[t]{4}{*}{$0.11 \%$} & ${ }_{56}^{130} \mathrm{Ba} \rightarrow{ }_{54}^{130} \mathrm{Xe}^{*}$ & $\left(1^{+}\right)$ & & 29 & 110 & 210 & 3 & 5.45 & 0.18 & $10^{-2}$ & $8 \times$ & $8 \times 10^{32}$ \\
\hline & & $\left(1^{+}\right)$ & $2544.43 \pm$ & $-39.1=$ & 110 & 210 & 6 & 5.45 & 0.18 & $1.4 \times 10^{-2}$ & $5 \times 10^{32}$ & $5 \times 10^{32}$ \\
\hline & & $\left(1^{+}\right)$ & $2628.360 \pm 0.001$ & $44.8 \pm$ & 110 & 210 & 34.56 & 5.45 & 0.18 & $1.4 \times 10^{-2}$ & $8 \times 10^{32}$ & $8 \times 10^{32}$ \\
\hline & & $\left(1^{+}\right)$ & $2637.50 \pm 0.05$ & 53.9 & 110 & 210 & 34.56 & 5.45 & 0.18 & $1.4 \times 10^{-2}$ & $1 \times 10^{33}$ & $1 \times 10^{33}$ \\
\hline \multirow[t]{2}{*}{$0.06 \%$} & ${ }_{66}^{156} \mathrm{Dy} \rightarrow \frac{1}{6}$ & {$\left[0^{+}\right]$} & $1804 \pm 7$ & $-100.4 \pm 0.10$ & 110 & 110 & 50.24 & 50.24 & 1.14 & $4.5 \times 10^{-2}$ & $1 \times 10^{32}$ & $3 \times 10^{32}$ \\
\hline & & $0^{+}$ & $1851.239 \pm 0.007^{\mathrm{a}}$ & $-53.1=$ & 110 & 110 & 50.24 & 50.24 & 1.14 & $4.5 \times 10^{-2}$ & $5 \times 10^{31}$ & $5 \times 10^{31}$ \\
\hline \multirow[t]{2}{*}{$0.14 \%$} & ${ }_{68}^{162} \mathrm{Er} \rightarrow{ }_{6}^{1}$ & $0^{+}$ & $1666.27 \pm 0.20$ & $-72.0 \pm 0.30$ & 110 & 110 & 53.79 & 53.79 & 1.19 & $5.1 \times 10^{-2}$ & $5 \times 10^{31}$ & $8 \times 10^{31}$ \\
\hline & & $1^{+}$ & $1745.716 \pm 0.007$ & $-38.2 \pm 0.30$ & 110 & 210 & 53.79 & 9.05 & 0.25 & $3.0 \times 10^{-2}$ & $3 \times 10^{31}$ & $3 \times 10^{31}$ \\
\hline $1.601 \%$ & ${ }_{68}^{164} \mathrm{Er} \rightarrow{ }_{66}^{164} \mathrm{Dy}^{*}$ & $0^{+}$ & 0 & $38.0 \pm 0.12$ & 110 & 210 & 53.79 & 9.05 & 0.31 & $3.0 \times 10^{-2}$ & $1 \times 10^{32}$ & $1 \times 10^{32}$ \\
\hline \multirow[t]{2}{*}{$0.014 \%$} & ${ }_{78}^{190} \mathrm{Pt} \rightarrow{ }_{76}^{190} \mathrm{Os}^{* *}$ & 1,2 & $1326.9 \pm 0.5^{\mathrm{a}}$ & $-0.1 \pm 0.47$ & 110 & 410 & 73.87 & 0.65 & 0.07 & $5.2 \times 10^{-2}$ & $3 \times 10^{25}$ & $2 \times 10^{29}$ \\
\hline & & & & -0.2 & 110 & 411 & 73.87 & 0.55 & 0.04 & $5.1 \times 10^{-2}$ & $5 \times 10^{26}$ & $3 \times 10^{30}$ \\
\hline
\end{tabular}

${ }^{\mathrm{a} C h a n n e l ~ w i t h ~ a ~ k n o w n ~ N M E ~ l i s t e d ~ i n ~ T a b l e ~ I V . ~}$

by Krivoruchenko and Tyrin (2020). The nucleon and nonnucleon spin-isospin correlations and the renormalization effects of the axial-vector coupling $g_{A}$ were discussed by Ejiri, Suhonen, and Zuber (2019).

The value $\Delta \epsilon_{\alpha \beta}^{*}$ shown in Tables XI and XII is a correction to the two-hole excitation energy (4.37). In the lowest approximation, $\Delta \epsilon_{\alpha \beta}^{*}$ is the Coulomb interaction energy of the holes. For the estimates reported here, the values of $\Delta \epsilon_{\alpha \beta}^{*}$ are determined empirically from Auger-electron spectroscopy, as described in Sec. IV.B. If no experimental data are available, $\Delta \epsilon_{\alpha \beta}^{*}$ are determined from calculations using the GRASP $2 \mathrm{~K}$ package. The calculations are performed for noble gas atoms with the simplest electron shell structure. For the remaining atoms with identical quantum numbers for the holes, the interaction energy is obtained via interpolation with a power function $\epsilon_{\alpha \beta}^{*} \sim a Z^{b}$. Tables XI and XII report the minimum and maximum normalized values of the half-lives. The confidence interval of the $99 \%$ probability is determined by the uncertainty in the degeneracy parameter of the parent and daughter atoms $M_{A, Z-2}^{* *}-M_{A, Z} \pm 2.6 \sigma$, where $\sigma^{2}=$ $(\Delta Q)^{2}+\left(\Delta E^{*}\right)^{2}$ and $\Delta Q$ and $\Delta E^{*}$ are the errors in the $Q_{2 \mathrm{EC}}$ value and the excitation energy $E^{*}=M_{A, Z-2}^{*}-M_{A, Z-2}$ of the daughter nuclide, respectively. If this interval includes zero, then at $99 \%$ C.L. the resonance is not excluded so that $\tilde{T}_{1 / 2}^{\min }$ gives the unitary limit.

In addition to the perspective nuclear pairs discussed by Krivoruchenko et al. (2011), the $Q_{2 \mathrm{EC}}$ values of other nuclide pairs were also measured. Experimental limits on the halflives of the $0 \nu 2 \mathrm{EC}$ decays of some other nuclei were previously known or established in recent years. These additional cases are analyzed on the same grounds; the normalized half-life estimates are presented in Table XII. Decays with a minimum normalized half-life of more than
$10^{34} \mathrm{yr}$ are not listed. Tables XI and XII thus report all interesting cases of the atoms for which $Q_{2 \mathrm{EC}}$ values were measured or the experimental limits on the $0 \nu 2 \mathrm{EC}$ halflives are available.

In the near-resonance region, one finds a group of excited levels of ${ }_{54}^{130} \mathrm{Xe}^{*}$ with unknown quantum numbers. We provide estimates for four excitation levels of ${ }_{54}^{130} \mathrm{Xe}^{*}$ with energies $2533.40,2544.43,2628.36$, and $2637.50 \mathrm{keV}$ of the assumed spin parity $1^{+}$. According to our estimates, these decays all appear to be nonresonant. The upper and lower limits of the normalized half-lives coincide with the accuracy under consideration because of small errors in the excitation energy of the daughter nucleus and the precise $Q_{2 \text { EC }}$ value of the pair. The same remark applies to the decays ${ }^{136} \mathrm{Ce} \rightarrow{ }^{136} \mathrm{Ba}^{* *}(2399.9),{ }^{156} \mathrm{Dy} \rightarrow{ }^{156} \mathrm{Gd}^{* *}(1851.24),{ }^{162} \mathrm{Er} \rightarrow$ ${ }^{162} \mathrm{Dy}^{* *}(1745.72), \quad{ }^{180} \mathrm{~W} \rightarrow{ }^{180} \mathrm{Hf}^{*}, \quad$ the decay ${ }^{156} \mathrm{Dy} \rightarrow$ ${ }^{156} \mathrm{Gd}^{* *}(1988.5)$ accompanied by the $K K$ or $K L_{1}$ capture, and some other decays.

The decay of ${ }^{152} \mathrm{Gd}$ is not resonant at $99 \%$ C.L., but its probability remains sufficiently high, with a normalized half-life of $3 \times 10^{28}-3 \times 10^{29} \mathrm{yr}$. The case of ${ }^{156} \mathrm{Dy}$, which decays into an excited state of gadolinium with energy $1988.5 \mathrm{keV}$, is noteworthy. The error in the $Q_{2 \mathrm{EC}}$ value of this pair is $100 \mathrm{eV}$; the resonance capture of electrons from the $L_{1} L_{1}$ state with a normalized half-life of $8 \times 10^{25} \mathrm{yr}$ cannot be excluded at $99 \%$ C.L. The NME for decay into the excited level $J^{\pi}=0^{+}$and the energy of $1851.239 \mathrm{keV}$ according to Kotila, Barea, and Iachello (2014) is equal to 0.35 (Table IV). Taking the same NME value for $1988.5 \mathrm{keV}$, one finds the half-life of $1.1 \times 10^{28} \mathrm{yr}$, which is even less than the half-life of ${ }^{152} \mathrm{Gd}$. For other cases considered in Tables XI and XII, the minimum values of the normalized half-life are above $10^{29} \mathrm{yr}$. 


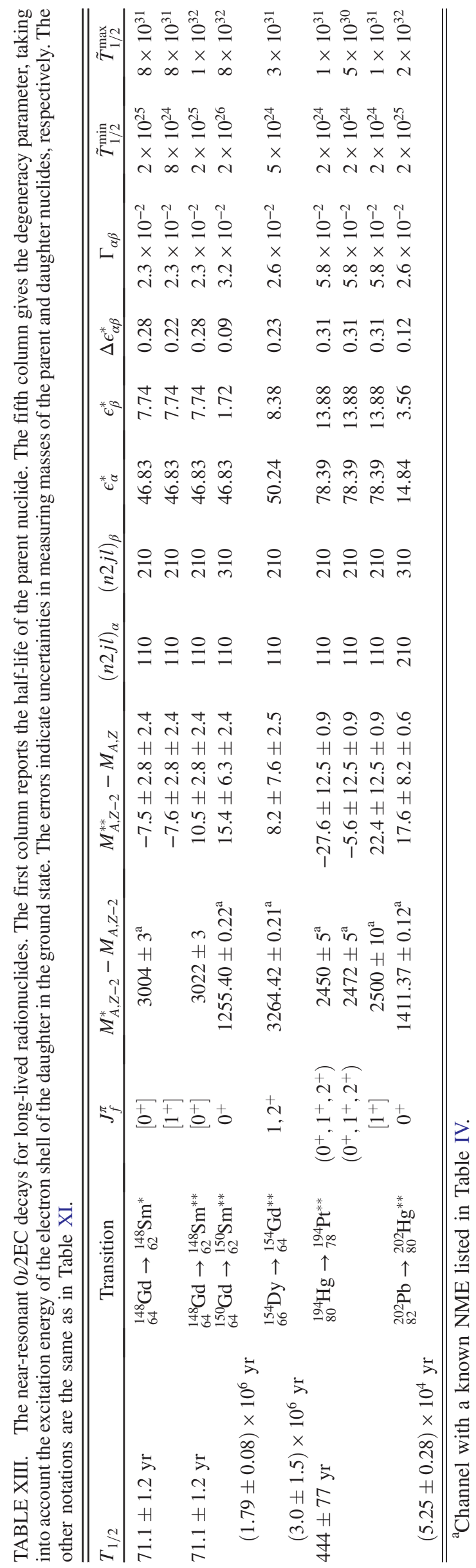

\section{B. Decays of long-lived radionuclides}

The radioactive elements are challenging to address experimentally; if one chooses the longest-living ones, then the experimental difficulties in working with such substances may be minimized. We performed an analysis of weakly radioactive elements with lifetimes of longer than a year using the Brookhaven National Laboratory database. From the point of view of resonant capture, two isotopes of gadolinium and isotopes of mercury and lead are of interest. The half-life estimates for these elements are presented in Table XIII. The $Q_{2 \mathrm{EC}}$ values of the pairs are not well known; thus, column 5 reports two errors in the masses of the parent and daughter nuclei. Column 4 shows the error in the excitation energy of the daughter nuclide. The first column gives the half-life of the radioactive parent nuclei with respect to the dominant decay. At the present level of knowledge of the parameters of the long-lived radionuclides, any of the channels listed in Table XIII can be exactly resonant.

\section{CONCLUSIONS}

Neutrino physics is a field of science characterized by a wealth of ideas, a multitude of unsolved problems, and mysteries that intrigue the imagination. Despite the more than half a century that has passed since the discovery of this particle, it remains the least understood among the fermions of the standard model. Great hope is placed on the study of $0 \nu 2 \beta^{-}$decay able to shed light on the type of neutrinos (Dirac or Majorana) and on the total lepton number conservation. However, numerous experimental attempts to observe $0 \nu 2 \beta^{-}$decay have been unsuccessful thus far.

In the steady stream of efforts devoted to the study of $2 \beta^{-}$decay, the reverse process of $2 \mathrm{EC}$ was in the shadows until recently. In this review, a comprehensive characterization of this process is presented, with a main focus on its neutrinoless mode, which can give the same information about the properties of the neutrino as the $0 \nu 2 \beta^{-}$decay. All aspects, both theoretical and experimental, revealing in detail unusual physical phenomena associated with the manifestations of the properties of mysterious neutrinos are considered.

To determine the possibility of observing the neutrinoless 2EC process in real experimental conditions, the lifetimes of nuclides in which the resonant conditions can occur were calculated. For realistic estimates it is necessary to describe properties of electron shells of atoms and 2EC nuclear matrix elements.

Atomic electron shells are involved in the $0 \nu 2 \mathrm{EC}$ process through the overlap of the electron wave functions with the nucleus and the excitation energy of electron shells. The short-distance electron wave functions are well defined in the framework of the multielectron DiracHartree-Fock schemes. Valence electrons are bound by several $\mathrm{eV}$, so the excitation energy of the shells is close to the double ionization potential. Experimental progress in atomic spectroscopy made it possible to study multiple ionization processes. Analysis of theoretical uncertainties and comparison with the Auger spectroscopic data leads to 
the conclusion that the excitation energies of $0 \nu 2 \mathrm{EC}$ can be determined with an accuracy of $60 \mathrm{eV}$ or better for heavy atoms, which is comparable to the accuracy in measuring the atomic masses in Penning traps and the deexcitation width of electron shells. An improvement of theoretical schemes or direct measurements of the excitation energy of electron shells with quantum numbers relevant for the $0 \nu 2 \mathrm{EC}$ process is of great interest.

The nuclear-theory frameworks used to evaluate the nuclear wave functions involved in the nuclear matrix elements of the resonant neutrinoless 2EC transitions cover theories based on the quasiparticle random-phase approximation (both spherical and deformed QRPAs), boson mapping (IBM-2), or modern EDFs. The last two base the matrixelement computations on the closure approximation, and only the QRPA-based theories avoid the use of closure. On the other hand, the IBM-2 and EDFs flexibly take into account the deformation degree of freedom, as also to a certain extent the deformed QRPA does.

The most straightforward are the matrix elements involved in the ground-state-to-ground-state captures in the deformed nuclei ${ }^{152} \mathrm{Gd},{ }^{156} \mathrm{Dy},{ }^{164} \mathrm{Er}$, and ${ }^{180} \mathrm{~W}$. For these nuclides the various theory frameworks (QRPA, IBM-2, EDF) give consistent results within a factor of 2 to 3 for the values of the nuclear matrix elements. Theoretical treatment of the excited nuclear resonant states, particularly those with high excitation energies, is a challenge. The problem is the identification of the theoretical state that corresponds to the resonant experimental state of a certain spin parity $J^{\pi}$, particularly when there are several experimental and theoretical states close to the resonant state. The intrinsic properties of these close-lying states can vary strongly from one state to the next, so selecting the proper state is essential for a reliable prediction of the 2EC nuclear matrix element. This effect is magnified in deformed nuclei, with possible coexisting structures at approximately similar excitation energies.

At the quark-lepton level, the underlying physical LNV mechanisms of the $0 \nu 2 \mathrm{EC}, 0 \nu \mathrm{EC} \beta^{+}, 0 \nu 2 \beta^{+}$, and $0 \nu 2 \beta^{-}$ processes are essentially the same. In the standard model represented by the sector of renormalized dimension-4 interactions the total number of leptons $L$ is conserved. The corresponding $\Delta L=2$ contributions can appear via nonrenormalizable effective operators of higher dimensions. We specified all the operators up to dimension 9 and discussed their possible high-scale origin from renormalizable theories. We presented in some detail three popular large-scale scenarios beyond the standard model. The conventional mechanism of Majorana neutrino exchange is highlighted by the fact that the corresponding operator has a minimal dimension $d=5$.

The effective electron neutrino Majorana mass $m_{\beta \beta}$ determines the amplitudes of $0 \nu 2 \beta^{-}$decay and the $0 \nu 2 \mathrm{EC}$ process. We reported on the lower and upper half-life limits of $0 \nu 2 \mathrm{EC}$ for near-resonant nuclides with the known nuclear matrix elements for the effective electron neutrino Majorana mass of $\left|m_{\beta \beta}\right|=100 \mathrm{meV}$.

The experimental sensitivity to the $0 \nu 2 \mathrm{EC}$ process is currently lower than that of the $0 \nu 2 \beta^{-}$decay. The strongest $0 \nu 2 \mathrm{EC}$ half-life limits are approximately $T_{1 / 2} \sim 10^{21}-10^{22} \mathrm{yr}$, while the $0 \nu 2 \beta^{-}$experiments have already achieved the sensitivity level of $\lim T_{1 / 2} \sim 10^{24}-10^{26} \mathrm{yr}$. The highest sensitivity to the $0 \nu 2 \mathrm{EC}$ process to date has been achieved using quite diverse experimental techniques: gaseous $\left({ }^{78} \mathrm{Kr}\right)$, scintillation $\left({ }^{106} \mathrm{Cd}\right)$ and cryogenic scintillating bolometric detectors $\left({ }^{40} \mathrm{Ca}\right)$, HPGe $\gamma$ spectrometry $\left({ }^{36} \mathrm{Ar},{ }^{58} \mathrm{Ni},{ }^{96} \mathrm{Ru}\right.$, $\left.{ }^{112} \mathrm{Sn}\right)$, and geochemical methods $\left({ }^{130} \mathrm{Ba},{ }^{132} \mathrm{Ba}\right)$.

The prospects for finding $0 \nu 2 \mathrm{EC}$ become more favorable if a resonance effect in double-electron capture occurs, a phenomenon that is peculiar to $0 \nu 2 \mathrm{EC}$. The resonant $0 \nu 2 \mathrm{EC}$ effect is expected to be clearly identified thanks to the high accuracy of the $\gamma$-quanta energies expected in the decay, while the background due to the neutrino accompanied decay ( $\mathrm{x}$ ray with energies up to several tens of $\mathrm{keV}$ ) never plays a role in practice (in contrast to the $0 \nu 2 \beta^{-}$experiments, where background caused by the $2 \nu$ mode becomes dominant due to poor energy or time resolution). The experimental sensitivity can be significantly improved by increasing the amount of isotopes of interest and utilization of enriched materials, by increasing the detection efficiency, reducing the background, and providing the highest possible energy resolution. HPGe detectors and low-temperature bolometers appear to be the most suitable detection techniques for $0 \nu 2 \mathrm{EC}$ experiments, with a sensitivity of $T_{1 / 2} \sim 10^{25}-10^{26} \mathrm{yr}$. Moreover, the complicated signature of the resonant effect can be a definite advantage, since the energies of the $\gamma$ quanta expected in most decays are tabulated and usually known with a high accuracy, which will ensure reliable identification of the effect.

The resonance in 2EC is associated with the degeneracy of mother and intermediate daughter atomic states that can in principle be fulfilled in 2EC only provided that the process is neutrinoless. These conditions cannot occur in the $2 \beta^{-}$decays. However, an insufficiently accurate knowledge of the atomic mass differences between the mother and daughter states has blocked the realization of this possibility thus far.

The development of PTMS has radically altered this situation. PTMS is superior to all other known methods of mass spectrometry. It has successfully been used for the determination of mass differences of nuclides with unprecedented low uncertainties down to the eV level. Mass differences for 19 such pairs connected via 2EC and listed in Tables VI and VII have been measured by PTMS, and new measurements are still planned for other pairs.

The refined analysis shows that at $90 \%$ C.L. none of the stable isotopes are exactly resonant when accompanied by electron capture from favorable states $n s_{1 / 2}$ and $n p_{1 / 2}$. Yet at 99\% C.L. it is impossible to exclude the exactly resonant character of the ${ }_{78}^{190} \mathrm{Pt} \rightarrow{ }_{73}^{190} \mathrm{Os}^{* *}$ decay to the excited state $1326.9 \pm 0.5 \mathrm{keV}$ of the daughter. For the vanishing degeneracy parameter and $J^{\pi}=1^{+}$, the half-life appears to be $3.3 \times 10^{26} \mathrm{yr}$. A more accurate knowledge of the $Q_{2 \mathrm{EC}}$ value, the excitation energy, and $J^{\pi}$ of ${ }_{76}^{190} \mathrm{Os}^{* *}$ would certainly be desirable. The ${ }_{66}^{156} \mathrm{Dy} \rightarrow{ }_{64}^{156} \mathrm{Gd}^{* *}$ decay to the excited state of $1988.5 \pm 0.2 \mathrm{keV}$ also demonstrates the proximity to the resonance. The decay half-life $1.1 \times 10^{28} \mathrm{yr}$ is within the $99 \%$ confidence interval. For the ground-state-to-ground-state transitions, the ${ }_{64}^{152} \mathrm{Gd} \rightarrow{ }_{62}^{152} \mathrm{Sm}^{*}$ decay with a lower halflife limit of $7 \times 10^{27} \mathrm{yr}$ is the most encouraging case. The 
long-lived radionuclides listed in Table XIII can be resonant at 90\% C.L.; however, production of these nuclides in significant amounts is technically complex.

The data suggest that with further refinement of the parameters the nuclides under discussion could become competitive with nuclides that decay through the $0 \nu 2 \beta^{-}$ channel, for which the lower half-life limit is already set at $\sim 10^{26} \mathrm{yr}$. This level of sensitivity is also achievable for $2 \mathrm{EC}$ processes. Moreover, since not all excited states of nuclides are known experimentally, other, not yet identified, nearresonant nuclides can also exist. Special demands are placed on the nuclear spectroscopy for the search of new relevant excited nuclear states and exact determination of the spin and parity values for them.

The search for nuclides satisfying the resonance condition in the $2 \mathrm{EC}$ process requires new experimental and theoretical efforts. The experimental side should focus on improving the accuracy of mass measurements by ion traps, the search for new excited states of nuclides in the resonance region, the determination of their excitation energies and quantum numbers, and the determination of the excitation energy of the electron shells using atomic spectroscopy methods. The theory should be aimed at refining theoretical schemes for calculating the $2 \mathrm{EC}$ halflife. Further progress requires the joint efforts of theorists in atomic, nuclear, and particle physics, as well as the development and implementation of advanced technologies that are already on the horizon. The double-electron capture process can prove to be an important player in the world beyond the standard model.

\section{LIST OF SYMBOLS AND ABBREVIATIONS}

$\begin{array}{ll}0 \nu 2 \beta^{-} & \text {neutrinoless double-beta decay } \\ 0 \nu 2 \mathrm{EC} & \text { neutrinoless double-electron capture } \\ 2 \mathrm{EC} & \text { double-electron capture } \\ \Gamma & \text { electromagnetic decay width } \\ \Delta & \text { degeneracy parameter }=Q \text { minus the } \\ & \text { total excitation energies of the nucleus } \\ & \text { and electron shells } \\ \epsilon^{*} & \text { excitation energy of electron shell } \\ \nu & \text { neutrino } \\ \nu_{i} & \text { ion frequencies in the trap } \\ A & \text { mass number } \\ \text { CC } & \text { charged current } \\ \text { C.L. } & \text { confidence level } \\ \text { DEIP } & \text { double-electron ionization potential } \\ E^{*} & \text { excitation energy of nucleus } \\ \text { EDF } & \text { energy-density functional } \\ \text { FWHM } & \text { full width at half maximum } \\ \text { GCM } & \text { Generating Coordinate Method } \\ \text { g.s. } & \text { ground state } \\ \text { GT } & \text { Gamow-Teller beta-decay type } \\ \text { HO } & \text { harmonic oscillator } \\ \text { HPGe } & \text { high purity germanium detector } \\ \text { IBFFM } & \text { interacting boson-fermion-fermion } \\ & \text { model }\end{array}$

\begin{tabular}{|c|c|}
\hline IBFFM-2 & proton-neutron IBFFM \\
\hline IBFM-2 & $\begin{array}{l}\text { microscopic interacting boson-fermion } \\
\text { model }\end{array}$ \\
\hline IBM & interacting boson model \\
\hline IBM-2 & microscopic IBM \\
\hline$K, L, M$, etc. & orbitals of atomic electrons \\
\hline LNV & lepton number violation \\
\hline LRSM & left-right symmetric model \\
\hline LQ & leptoquark \\
\hline$M$ & neutral atom mass value \\
\hline MCM & multiple-commutator model \\
\hline MCP & microchannel plate \\
\hline MT & measurement trap \\
\hline m. (w.e.) & meters of water equivalent \\
\hline NME & nuclear matrix element \\
\hline PI-ICR & phase-image ion-cyclotron resonance \\
\hline PMNS & $\begin{array}{l}\text { Pontecorvo-Maki-Nakagawa-Sakata } \\
\text { mixing matrix }\end{array}$ \\
\hline PT & preparation trap \\
\hline PTMS & Penning-trap mass spectrometry \\
\hline$Q$ & $\begin{array}{l}\text { value-mass difference between neutral } \\
\text { parent and daughter atoms }\end{array}$ \\
\hline QCD & quantum chromodynamics \\
\hline QED & quantum electrodynamics \\
\hline QRPA & $\begin{array}{l}\text { quasiparticle random-phase approxi- } \\
\text { mation }\end{array}$ \\
\hline RGE & renormalization group equation \\
\hline SEIP & single-electron ionization potential \\
\hline SM & standard model \\
\hline SSB & spontaneous symmetry breaking \\
\hline SUSY & supersymmetric model \\
\hline RPV & $R$-parity violating \\
\hline$T_{1 / 2}$ & radioactive decay half-life \\
\hline $\begin{array}{l}\text { TOF-ICR } \\
Z\end{array}$ & $\begin{array}{l}\text { time-of-flight ion-cyclotron resonance } \\
\text { atomic number }\end{array}$ \\
\hline
\end{tabular}

\section{ACKNOWLEDGMENTS}

We appreciate the valuable discussions with M. Block, T. Eronen, Amand Faessler, D. Frekers, V. Shabaev, F. Šimkovic, and I. Tupitsyn on topics related to 2EC physics. This work is supported in part by the Max Planck Society. K. B. and S. E. acknowledge the support of the European Research Council (ERC) under the European Union's Horizon 2020 research and innovation program under Grant Agreement No. 832848FunI. F. A. D. and V. I. T. were supported in part by National Research Foundation of Ukraine Grant No. 2020.02/0011. S. K. acknowledges the support of ANID Fondecyt (Chile) Grant No. 1190845 and CONICYT (Chile) Project No. PIA/ Basal FB0821. M. I. K. is supported by RFBR Grant No. 1802-00733 (Russia) and the Alexander von Humboldt Foundation (Germany). Yu. N. N. is supported by the Extreme Matter Institute EMMI (GSI Darmstadt). J. S. was partly supported by the Academy of Finland under Project 
No. 318043. F. A. D., M. I. K., Yu. N. N., and V. I. T. acknowledge the kind hospitality of Max-Planck-Institut für Kernphysik in Heidelberg, where part of this work was done.

\section{REFERENCES}

Abe, K., et al., 2015, "Search for $n-\bar{n}$ oscillation in SuperKamiokande," Phys. Rev. D 91, 072006.

Abe, K., et al., 2018, "Improved search for two-neutrino double electron capture on ${ }^{124} \mathrm{Xe}$ and ${ }^{126} \mathrm{Xe}$ using particle identification in XMASS-I,” Prog. Theor. Exp. Phys. 2018, 053 D03.

Abgrall, N., et al., 2017, "The large enriched germanium experiment for neutrinoless double beta decay (LEGEND)," AIP Conf. Proc. 1894, 020027.

Aghanim, N., et al., 2018, "Planck 2018 results. VI. Cosmological parameters," arXiv:1807.06209.

Agostini, M., et al., 2016, "Limit on the radiative neutrinoless double electron capture of ${ }^{36} \mathrm{Ar}$ from GERDA Phase I,” Eur. Phys. J. C 76, 652.

Agostini, M., et al., 2018, "Improved Limit on Neutrinoless Double- $\beta$ Decay of ${ }^{76} \mathrm{Ge}$ from GERDA Phase II," Phys. Rev. Lett. 120, 132503.

Albert, J. B., et al., 2018, "Search for Neutrinoless Double-Beta Decay with the Upgraded EXO-200 Detector," Phys. Rev. Lett. 120, 072701.

Alduino, C., et al., 2018a, "First Results from CUORE: A Search for Lepton Number Violation via $0 \nu 2 \beta$ Decay of ${ }^{130}$ Te," Phys. Rev. Lett. 120, 132501.

Alduino, C., et al., 2018b, "Search for neutrinoless $\beta^{+} \mathrm{EC}$ decay of ${ }^{120}$ Te with CUORE-0," Phys. Rev. C 97, 055502.

Alessandria, F., et al., 2012, "CUORE crystal validation runs: Results on radioactive contamination and extrapolation to CUORE background," Astropart. Phys. 35, 839-849.

Anastasio, M. R., L. S. Celenza, W. S. Pong, and C. M. Shakin, 1983, "Relativistic nuclear structure physics," Phys. Rep. 100, 327-392.

Andreotti, E., M. Hult, G. Marissens, R González de Orduña, and P. Vermaercke, 2012, "Study of the double beta decays of ${ }^{96} \mathrm{Ru}$ and ${ }^{104}$ Ru," Appl. Radiat. Isot. 70, 1985-1989.

Andreotti, E., et al., 2011, "Search for $\beta^{+} / \mathrm{EC}$ double beta decay of ${ }^{120}$ Te," Astropart. Phys. 34, 643-648.

Angloher, G., et al., 2016, "New limits on double electron capture of ${ }^{40} \mathrm{Ca}$ and ${ }^{180} \mathrm{~W}$," J. Phys. G 43, 095202.

Aprile, E., et al., 2017, "Search for two-neutrino double electron capture of ${ }^{124}$ Xe with XENON100," Phys. Rev. C 95, 024605.

Arbeláez, C., M. González, M. Hirsch, and S. G. Kovalenko, 2016, "QCD corrections and long-range mechanisms of neutrinoless double beta decay," Phys. Rev. D 94, 096014.

Arbeláez, C., M. González, S. G. Kovalenko, and M. Hirsch, 2017, "QCD-improved limits from neutrinoless double beta decay," Phys. Rev. D 96, 015010.

Arnold, R., et al., 2015, "Results of the search for neutrinoless double- $\beta$ decay in ${ }^{100}$ Mo with the NEMO-3 experiment," Phys. Rev. D 92, 072011.

Aunola, M., J. Suhonen, A. S. Barabash, V. I. Umatov, R. Gurrián, F. Hubert, and Ph. Hubert, 1995, "Double beta processes in ${ }^{92} \mathrm{Mo}$," JETP Lett. 62, 706-709, http://www.jetpletters.ac.ru/ps/1220/ article_18440.shtml.

Avignone III, F. T., S. R. Elliott, and J. Engel, 2008, "Double beta decay, Majorana neutrinos, and neutrino mass," Rev. Mod. Phys. 80, 481-516.

Ayala, C., and G. Cvetic, and L. Gonzalez, 2020, "Evaluation of neutrinoless double beta decay: QCD running to sub-GeV scales," Phys. Rev. D 101, 094003.
Azzolini, O., et al., 2018, "First Result on the Neutrinoless Double- $\beta$ Decay of ${ }^{82}$ Se with CUPID-0," Phys. Rev. Lett. 120, 232502.

Band, I. M., and M. B. Trzhaskovskaya, 1986, "Electron-wavefunction expansion amplitudes near the origin calculated in the Dirac-Fock-Slater and Dirac-Fock potentials," At. Data Nucl. Data Tables 35, 1-13.

Barabash, A. S., 2018, "Main features of detectors and isotopes to investigate double beta decay with increased sensitivity," Int. J. Mod. Phys. A 33, 1843001.

Barabash, A. S., R. Gurriarán, E. Hubert, Ph. Hubert, and V. L. Umatov, 1997, "Improved limits on double beta processes in ${ }^{92} \mathrm{Mo}$," Z. Phys. A 357, 351-352.

Barabash, A. S., F. Hubert, Ph. Hubert, and V. Umatov, 2007, "New limits on the $\beta^{+}$EC and ECEC processes in ${ }^{120}$ Te," J. Phys. G 34, 1721-1728.

Barabash, A. S., Ph. Hubert, Ch. Marquet, A. Nachab, S. I. Konovalov, F. Perrot, F. Piquemal, and V. Umatov, 2011, "Improved limits on $\beta^{+} \mathrm{EC}$ and ECEC processes in ${ }^{112} \mathrm{Sn}$," Phys. Rev. C 83, 045503.

Barabash, A. S., Ph. Hubert, A. Nachab, S. I. Konovalov, and V. Umatov, 2009, "Search for $\beta^{+}$EC and ECEC processes in ${ }^{112} \mathrm{Sn}$," Phys. Rev. C 80, 035501.

Barabash, A. S., and Ph. Hubert, A. Nachab, S. I. Konovalov, I. A. Vanyushin, and V. Umatov, 2008, "Search for $\beta^{+}$EC and ECEC processes in ${ }^{112} \mathrm{Sn}$ and $\beta^{-} \beta^{-}$decay of ${ }^{124} \mathrm{Sn}$ to the excited states of ${ }^{124}$ Te," Nucl. Phys. A807, 269-281.

Barabash, A. S., Ph. Hubert, A. Nachab, and V. Umatov, 2007, "Search for $\beta^{+}$EC and ECEC processes in ${ }^{74}$ Se," Nucl. Phys. A785, 371-380.

Barabash, A.S., and V. V. Kuzminov, V. M. Lobashev, V. M. Novikov, B. M. Ovchinnikov, and A. A. Pomanskyl, 1989, "Results of the experiment on the search for double beta decay of ${ }^{136} \mathrm{Xe}$, ${ }^{134} \mathrm{Xe}$ and ${ }^{124} \mathrm{Xe}$," Phys. Lett. B 223, 273-276.

Barabash, A. S., and R. R. Saakyan, 1996, "Experimental limits on $2 \beta^{+}, K \beta^{+}$, and $2 K$ processes for ${ }^{130} \mathrm{Ba}$ and on $2 K$ capture for ${ }^{132}$ Ba," Phys. At. Nucl. 59, 179-184.

Barabash, A. S., V. I. Umatov, R. Gurriarán, F. Hubert, Ph. Hubert, M. Aunola, and J. Suhonen, 1996, "Theoretical and experimental investigation of the double beta processes in ${ }^{106} \mathrm{Cd}$," Nucl. Phys. A604, 115-128.

Barabash, A. S., et al., 2020, "Improved limits on $\beta^{+}$EC and ECEC processes in ${ }^{74}$ Se," Nucl. Phys. A996, 121697.

Barinov, V., V. Gavrin, V. Gorbachev, D. Gorbunov, and T. Ibragimova, 2019, "BEST potential in testing the eV-scale sterile neutrino explanation of reactor antineutrino anomalies," Phys. Rev. D 99, 111702(R).

Belli, P., R. Bernabei, F. Cappella, R. Cerulli, F. A. Danevich, A. d'Angelo, A. Di Marco, A. Incicchitti, F. Nozzoli, and V. I. Tretyak, 2011a, "Search for $2 \beta$ decay of cerium isotopes with $\mathrm{CeCl}_{3}$ scintillator," J. Phys. G 38, 015103.

Belli, P., R. Bernabei, F. Cappella, R. Cerulli, F. A. Danevich, S. d'Angelo, A. Incicchitti, V. V. Kobychev, D. V. Poda, and V. I. Tretyak, 2011b, "Final results of an experiment to search for $2 \beta$ processes in zinc and tungsten with the help of radiopure $\mathrm{ZnWO}_{4}$ crystal scintillators," J. Phys. G 38, 115107.

Belli, P., R. Bernabei, S. d'Angelo, F. Cappella, R. Cerulli, A. Incicchitti, M. Laubenstein, D. Prosperi, and V. I. Tretyak, 2009, "First limits on neutrinoless resonant $2 \varepsilon$ captures in ${ }^{136} \mathrm{Ce}$ and new limits for other $2 \beta$ processes in ${ }^{136} \mathrm{Ce}$ and ${ }^{138} \mathrm{Ce}$ isotopes," Nucl. Phys. A824, 101-114.

Belli, P., R. Bernabei, A. Incicchitti, C. Arpesella, V. V. Kobychev, O. A. Ponkratenko, V. I. Tretyak, and Yu. G. Zdesenko, 1999, "New limits on $2 \beta^{+}$decay processes in ${ }^{106} \mathrm{Cd}$," Astropart. Phys. 10, 115-120. 
Belli, P., et al., 1999, "New limits on spin-dependent coupled WIMPs and on $2 \beta$ processes in ${ }^{40} \mathrm{Ca}$ and ${ }^{46} \mathrm{Ca}$ by using low radioactive $\mathrm{CaF}_{2}(\mathrm{Eu})$ crystal scintillators," Nucl. Phys. B563, 97-106.

Belli, P., et al., 2003, "Performances of a $\mathrm{CeF}_{3}$ crystal scintillator and its application to the search for rare processes," Nucl. Instrum. Methods Phys. Res., Sect. A 498, 352-361.

Belli, P., et al., 2008a, "Search for $2 \beta$ processes in ${ }^{64} \mathrm{Zn}$ with the help of $\mathrm{ZnWO}_{4}$ crystal scintillator," Phys. Lett. B 658, 193-197.

Belli, P., et al., 2008b, "Search for double- $\beta$ decay processes in ${ }^{108} \mathrm{Cd}$ and ${ }^{114} \mathrm{Cd}$ with the help of the low-background $\mathrm{CdWO}_{4}$ crystal scintillator," Eur. Phys. J. A 36, 167-170.

Belli, P., et al., 2009a, "Search for double beta decay of zinc and tungsten with low background $\mathrm{ZnWO}_{4}$ crystal scintillators," Nucl. Phys. A826, 256-273.

Belli, P., et al., 2009b, "Search for double- $\beta$ decays of ${ }^{96} \mathrm{Ru}$ and ${ }^{104}$ Ru by ultra-low background HPGe $\gamma$ spectrometry," Eur. Phys. J. A 42, 171.

Belli, P., et al., 2010, "Development of enriched ${ }^{106} \mathrm{CdWO}_{4}$ crystal scintillators to search for double $\beta$ decay processes in ${ }^{106} \mathrm{Cd}$," Nucl. Instrum. Methods Phys. Res., Sect. A 615, 301-306.

Belli, P., et al., 2011a, "First search for double $\beta$ decay of dysprosium," Nucl. Phys. A859, 126-139.

Belli, P., et al., 2011b, "First search for double- $\beta$ decay of platinum by ultra-low background HPGe $\gamma$ spectrometry," Eur. Phys. J. A 47, 91.

Belli, P., et al., 2012a, "Radioactive contamination of $\operatorname{SrI}_{2}(\mathrm{Eu})$ crystal scintillator," Nucl. Instrum. Methods Phys. Res., Sect. A 670, 10-17.

Belli, P., et al., 2012b, "Search for double- $\beta$ decay processes in ${ }^{106} \mathrm{Cd}$ with the help of a ${ }^{106} \mathrm{CdWO}_{4}$ crystal scintillator," Phys. Rev. C 85, 044610.

Belli, P., et al., 2013a, "First search for double- $\beta$ decay of ${ }^{184}$ Os and ${ }^{192}$ Os," Eur. Phys. J. A 49, 24.

Belli, P., et al., 2013b, "Search for $2 \beta$ decays of ${ }^{96} \mathrm{Ru}$ and ${ }^{104} \mathrm{Ru}$ by ultralow-background HPGe $\gamma$ spectrometry at LNGS: Final results," Phys. Rev. C 87, 034607.

Belli, P., et al., 2014, "Search for double beta decay of ${ }^{136} \mathrm{Ce}$ and ${ }^{138}$ Ce with HPGe gamma detector," Nucl. Phys. A930, 195-208.

Belli, P., et al., 2016, "Search for double- $\beta$ decay in ${ }^{106} \mathrm{Cd}$ with an enriched ${ }^{106} \mathrm{CdWO}_{4}$ crystal scintillator in coincidence with four HPGe detectors," Phys. Rev. C 93, 045502.

Belli, P., et al., 2017, "New limits on $2 \varepsilon, \epsilon \beta^{+}$and $2 \beta^{+}$decay of ${ }^{136} \mathrm{Ce}$ and ${ }^{138} \mathrm{Ce}$ with deeply purified cerium sample," Eur. Phys. J. A 53, 172.

Belli, P., et al., 2018, "First search for $2 \varepsilon$ and $\epsilon \beta^{+}$decay of ${ }^{162} \mathrm{Er}$ and new limit on $2 \beta^{-}$decay of ${ }^{170} \mathrm{Er}$ to the first excited level of ${ }^{170}$ Yb," J. Phys. G 45, 095101.

Belli, P., et al., 2019a, "First direct search for $2 \varepsilon$ and $\varepsilon \beta^{+}$decay of ${ }^{144} \mathrm{Sm}$ and $2 \beta^{-}$decay of ${ }^{154} \mathrm{Sm}$," Eur. Phys. J. A 55, 201.

Belli, P., et al., 2019b, "First search for $2 \varepsilon$ and $\varepsilon \beta^{+}$processes in ${ }^{168}$ yb," Nucl. Phys. A990, 64-78.

Belli, P., et al., 2020, "Search for double beta decay of ${ }^{106} \mathrm{Cd}$ with an enriched ${ }^{106} \mathrm{CdWO}_{4}$ crystal scintillator in coincidence with $\mathrm{CdWO}_{4}$ scintillation counters," Universe 6, 182.

Bellotti, E., E. Fiorini, C. Liguori, A. Pullia, A. Sarracino, and L. Zanotti, 1982, "An experimental investigation on lepton number conservation in double-beta processes," Lett. Nuovo Cimento 33, 273.

Berestetsky, V. B., E. M. Lifshitz, and L. P. Pitaevsky, 1982, Quantum Electrodynamics (Pergamon, Oxford).

Berger, J. F., M. Girod, and D. Gogny, 1984, "Microscopic analysis of collective dynamics in low energy fission," Nucl. Phys. A428, 23-36.
Berlovich, E. E., and Yu. N. Novikov, 1970, "Probability of double beta decay of nuclei in regions far removed from the beta-stable band," Sov. Phys. Dokl. 14, 986-988.

Bernabei, R., P. Belli, A. Incicchitti, D. Prosperi, C. Bacci, F. De Notaristefani, G. J. Davies, and C. J. Dai, 1997, "Feasibility of $\beta \beta$ decay searches with $\mathrm{Ce}$ isotopes using $\mathrm{CeF}_{3}$ scintillators," Nuovo Cimento Soc. Ital. Fis. 110A, 189-195.

Bernabeu, J., A. De Rujula, and C. Jarlskog, 1983, "Neutrinoless double electron capture as a tool to measure the electron neutrino mass," Nucl. Phys. B223, 15-28.

Bernabéu, J., and A. Segarra, 2018, "Stimulated transitions in resonant atom Majorana mixing," J. High Energy Phys. 02, 017.

Berthelot, A., R. Chaminade, C. Levi, and L. Papineau, 1953, "Recherche d'une double capture K dans le zinc (64)," C.R. Phys. 236, 1769.

Bialynicki-Birula, I., 1970, "Renormalization, diagrams, and gauge invariance," Phys. Rev. D 2, 2877-2886.

Bikit, I., M. Krmar, J. Slivka, I. Aničin, M. Veskovic, and Lj. Čonkič, 1995, "Electron-positron conversion decay of ${ }^{64} \mathrm{Zn}$," Appl. Radiat. Isot. 46, 455-456.

Bikit, I., M. Krmar, J. Slivka, M. Vesković, Lj. Čonkić, and I. Aničinl, 1998, "New results on the double $\beta$ decay of iron," Phys. Rev. C 58, 2566.

Bikit, I., N. Zikić-Todorović, J. Slivka, M. Vesković, M. Krmar, Lj. Ćonkić, J. Puzović, and I. V. Aničin, 2003, "Double $\beta$ decay of ${ }^{50}$ Cr," Phys. Rev. C 67, 065801.

Bilenky, S., 2018, Introduction to the Physics of Massive and Mixed Neutrinos, 2nd ed., Lecture Notes in Physics Vol. 947 (Springer-Verlag, Berlin).

Bilenky, S. M., and C. Giunti, 2015, "Neutrinoless double-beta decay: A probe of physics beyond the standard model," Int. J. Mod. Phys. A 30, 1530001.

Bilenky, S. M., and S. T. Petcov, 1987, "Massive neutrinos and neutrino oscillations," Rev. Mod. Phys. 59, 671-754.

Bjorken, J. D., and S. D. Drell, 1965, Relativistic Quantum Fields (McGraw-Hill, New York).

Blaum, K., 2006, "High-accuracy mass spectrometry with stored ions," Phys. Rep. 425, 1-78.

Blaum, K., J. Dilling, and W. Nörtershäuser, 2013, "Precision atomic physics techniques for nuclear physics with radioactive beams," Phys. Scr. T152, 014017.

Block, M., et al., 2007, "Towards direct mass measurements of nobelium at SHIPTRAP," Eur. Phys. J. D 45, 39-45.

Bloxham, T., et al., 2007, "First results on double $\beta$-decay modes of $\mathrm{Cd}, \mathrm{Te}$, and Zn isotopes," Phys. Rev. C 76, 025501.

Bogoliubov, N. N., and D. V. Shirkov, 1980, Introduction to the Theory of Quantized Field, 3rd ed. (John Wiley \& Sons, New York).

Bohr, A., and B. R. Mottelson, 1969, Nuclear Structure, Vol. I (Benjamin, New York).

Bollen, G., et al., 1987, "First absolute mass measurements of shortlived isotopes," Hyperfine Interact. 38, 793-802.

Bonnet, F., M. Hirsch, T. Ota, and W. Winter, 2013, "Systematic decomposition of the neutrinoless double beta decay operator," J. High Energy Phys. 03, 55.

Brant, S., and V. Paar, 1988, "IBFFM yrast states in odd-odd nuclei associated with $\mathrm{O}(6)$ and $\mathrm{SU}(3)$ limits," Z. Phys. A 329, 151-159.

Breit, G., 1929, "The effect of retardation on the interaction of two electrons," Phys. Rev. 34, 553-573.

Brockmann, R., and R. Machleidt, 1984, "Nuclear saturation in a relativistic Brueckner-Hartree-Fock approach,” Phys. Lett. B 149, 283-287. 
Brown, B. A., 1992, "Simple relation for alpha decay half-lives," Phys. Rev. C 46, 811-814.

Brown, L. S., and G. Gabrielse, 1986, "Geonium theory: Physics of a single electron or ion in a Penning trap," Rev. Mod. Phys. 58, 233-311.

Broyles, A. A., 1988, "Relativistic equation for the multielectron atom," Phys. Rev. A 38, 1137-1148.

Buchmüller, W., R. Rückl, and D. Wyler, 1987, "Leptoquarks in lepton-quark collisions," Phys. Lett. B 191, 442-448.

Bukhner, E., et al., 1990, "Rare decays of mercury nuclei," Sov. J. Nucl. Phys. 52, 193-197.

Bunge, C. F., J. A. Barrientos, and A. V. Bunge, 1993, "RoothaanHartree-Fock ground-state atomic wave functions: Slater-type orbital expansions and expectation values for $Z=2-54$," At. Data Nucl. Data Tables 53, 113-162.

Bustabad, S., G. Bollen, M. Brodeur, D. L. Lincoln, S. J. Novario, M. Redshaw, R. Ringle, and S. Schwarz, 2013, "Examination of the possible enhancement of neutrinoless double-electron capture in ${ }^{78} \mathrm{Kr}$," Phys. Rev. C 88, 035502.

Campbell, J. L., and T. Papp, 2001, "Width of the atomic $K-N 7$ levels," At. Data Nucl. Data Tables 77, 1-56.

Cerulli, R., P. Belli, R. Bernabei, F. Cappella, F. Nozzoli, F. Montecchi, A. d'Angelo, A. Incicchitti, D. Prosperi, and C. J. Dai, 2004, "Performances of a $\mathrm{BaF}_{2}$ detector and its application to the search for $\beta \beta$ decay modes in ${ }^{130} \mathrm{Ba}$," Nucl. Instrum. Methods Phys. Res., Sect. A 525, 535-543.

Chaudhuri, A., et al., 2007, "Carbon-cluster mass calibration at SHIPTRAP,” Eur. Phys. J. D 45, 47-53.

Chernyak, D. M., F. A. Danevich, A. Giuliani, E. Olivieri, M. Tenconi, and V. I. Tretyak, 2012, "Random coincidence of $2 \nu 2 \beta$ decay events as a background source in bolometric $0 \nu 2 \beta$ decay experiments," Eur. Phys. J. C 72, 1989.

Chkvorets, O., 2008, "Search for double beta decay with HPGe detectors at the Gran Sasso underground laboratory," Ph.D. thesis (University of Heidelberg), arXiv:0812.1206.

Cirigliano, V., W. Dekens, J. de Vries, M. L Graesser, and E. Mereghetti, 2017a, "Neutrinoless double beta decay in chiral effective field theory: Lepton number violation at dimension seven," J. High Energy Phys. 12, 082.

Cirigliano, V., W. Dekens, J. de Vries, M.L Graesser, and E. Mereghetti, 2018a, "A neutrinoless double beta decay master formula from effective field theory," J. High Energy Phys. 12, 097.

Cirigliano, V., W. Dekens, J. De Vries, M. L. Graesser, E. Mereghetti, S. Pastore, M. Piarulli, U. Van Kolck, and R. B. Wiringa, 2019, "Renormalized approach to neutrinoless double- $\beta$ decay," Phys. Rev. C 100, 055504.

Cirigliano, V., W. Dekens, J. De Vries, M. L. Graesser, E. Mereghetti, S. Pastore, and U. Van Kolck, 2017b, "Neutrinoless double beta decay and chiral SU(3)," Phys. Lett. B 769, 460.

Cirigliano, V., W. Dekens, J. De Vries, M. L. Graesser, E. Mereghetti, S. Pastore, and U. Van Kolck, 2018b, "New Leading Contribution to Neutrinoless Double- $\beta$ Decay," Phys. Rev. Lett. 120, 202001.

Cirigliano, V., W. Detmold, A. Nicholson, and Ph. Shanahan, 2020, "Lattice QCD inputs for nuclear double beta decay," arXiv:2003 .08493 .

Clementi, E., and C. Roetti, 1974, "Roothaan-Hartree-Fock atomic wavefunctions: Basis functions and their coefficients for ground and certain excited states of neutral and ionized atoms, $Z \leq 54$," At. Data Nucl. Data Tables 14, 177-478.

Cremonesi, O., and M. Pavan, 2014, "Challenges in double beta decay,” Adv. High Energy Phys. 2014, 951432.
Cribier, M., et al., 1996, "Production of a $62 \mathrm{PBq}{ }^{51} \mathrm{Cr}$ low energy neutrino source for GALLEX," Nucl. Instrum. Methods Phys. Res., Sect. A 378, 233-250.

Danevich, F. A., M. Hult, D. V. Kasperovych, G. P. Kovtun, K. V. Kovtun, G. Lutter, G. Marissens, O. G. Polischuk, S. P. Stetsenko, and V. I. Tretyak, 2020, "First search for $2 \varepsilon$ and $\varepsilon \beta^{+}$decay of ${ }^{174} \mathrm{Hf}$ " (to be published).

Danevich, F. A., V. V. Kobychev, S. S. Nagorny, D. V. Poda, V. I. Tretyak, S. S. Yurchenko, and Yu. G. Zdesenko, 2005, "ZnWO crystals as detectors for $2 \beta$ decay and dark matter experiments," Nucl. Instrum. Methods Phys. Res., Sect. A 544, 553-564.

Danevich, F. A., V. V. Kobychev, O. A. Ponkratenko, V. I. Tretyak, and Yu. G. Zdesenko, 2001, "Quest for double beta decay of ${ }^{160} \mathrm{Gd}$ and Ce isotopes," Nucl. Phys. A694, 375-391.

Danevich, F. A., A. Sh. Georgadze, V. V. Kobychev, B. N. Kropivyansky, V. N. Kuts, A. S. Nikolayko, O. A. Ponkratenko, V. I. Tretyak, and Yu. G. Zdesenko, 1996, "Beta decay of ${ }^{113} \mathrm{Cd}$," Phys. At. Nucl. 59, 1-5.

Danevich, F. A., and V. I. Tretyak, 2018, "Radioactive contamination of scintillators," Int. J. Mod. Phys. A 33, 1843007.

Danevich, F. A., et al., 1996, "Investigation of $\beta^{+} \beta^{+}$and $\beta^{+} / \mathrm{EC}$ decay of ${ }^{106}$ Cd," Z. Phys. A 355, 433-437.

Danevich, F. A., et al., 2003, "Search for $2 \beta$ decay of cadmium and tungsten isotopes: Final results of the Solotvina experiment," Phys. Rev. C 68, 035501.

Darwin, C. G., 1920, "The dynamical motions of charged particles," London Edinburgh Dublin Philos. Mag. J. Sci. 39, 537-551.

Dawson, J., D. Degering, M. Köhler, R. Ramaswamy, C. Reeve, J. R. Wilson, and K. Zuber, 2008, "Search for double- $\beta$ decays of tin isotopes with enhanced sensitivity," Phys. Rev. C 78, 035503.

Dawson, J., R. Ramaswamy, C. Reeve, J. R. Wilson, and K. Zuber, 2008, "A search for various double beta decay modes of tin isotopes," Nucl. Phys. A799, 167-180.

Dawson, J. V., et al., 2009a, "Experimental study of double- $\beta$ decay modes using a CdZnTe detector array," Phys. Rev. C 80, 025502.

Dawson, J. V., et al., 2009b, "Experimental study of double- $\beta$ decay modes using a CdZnTe detector array," Phys. Rev. C 80, 025502.

Delion, D. S., and J. Suhonen, 2017, “Two-neutrino $\beta \beta$ decays and low-lying Gamow-Teller $\beta^{-}$strength functions in the mass range $A=70-176$," Phys. Rev. C 95, 034330.

Dell'Oro, S., S. Marcocci, M. Viel, and F. Vissani, 2016, "Neutrinoless double beta decay: 2015 review," Adv. High Energy Phys. 2016, 2162659.

Desclaux, J. P., 1973, "Relativistic Dirac-Fock expectation values for atoms with $Z=1$ to $Z=120$," At. Data Nucl. Data Tables 12, 311-406.

Diaz, C. G., 2013, "Characterization of scintillating bolometers for particle detection and installation of a bolometric test facility in the University of Zaragoza," Ph.D. thesis (University of Zaragoza).

Doi, M., T. Kotani, and E. Takasugi, 1985, "Double beta decay and Majorana neutrino," Prog. Theor. Phys. Suppl. 83, 1-175.

Dolinski, M. J., A. W. P. Poon, and W. Rodejohann, 2019, "Neutrinoless double-beta decay: Status and prospects," Annu. Rev. Nucl. Part. Sci. 69, 219-251.

Dover, C. B., A. Gal, and J. M. Richard, 1983, "Neutron-antineutron oscillations in nuclei," Phys. Rev. D 27, 1090-1100.

Dover, C. B., A. Gal, and J.M. Richard, 1985, "Limits on the neutron-antineutron oscillation time from the stability of nuclei," Phys. Rev. C 31, 1423-1429.

Droese, C., M. Block, M. Dworschak, S. Eliseev, E. Minaya Ramirez, D. Nesterenko, and L. Schweikhard, 2011, "Investigation of the magnetic field fluctuation and implementation of a temperature and 
pressure stabilization at SHIPTRAP," Nucl. Instrum. Methods Phys. Res., Sect. A 632, 157-163.

Droese, C., et al., 2012, "Probing the nuclide ${ }^{180} \mathrm{~W}$ for neutrinoless double-electron capture exploration," Nucl. Phys. A875, 1-7.

Dyall, K. G., I. P. Grant, C. T. Johnson, F. A. Parpia, and E. P. Blummer, 1989, "GRASP: A general-purpose relativistic atomic structure program," Comput. Phys. Commun. 55, 425-456.

Ebert, J., et al., 2013, "Current status and future perspectives of the COBRA experiment," Adv. High Energy Phys. 2013, 703572.

Ebert, J., et al., 2016a, "Results of a search for neutrinoless double$\beta$ decay using the COBRA demonstrator," Phys. Rev. C 94, 024603.

Ebert, J., et al., 2016b, "The COBRA demonstrator at the LNGS underground laboratory,' Nucl. Instrum. Methods Phys. Res., Sect. A 807, 114-120.

Eibach, M., G. Bollen, K. Gulyuz, C. Izzo, M. Redshaw, R. Ringle, R. Sandler, and A. A. Valverde, 2016, "Double resonant enhancement in the neutrinoless double-electron capture of ${ }^{190} \mathrm{Pt}$," Phys. Rev. C 94, 015502.

Ejiri, H., J. Suhonen, and K. Zuber, 2019, "Neutrino-nuclear responses for astro-neutrinos, single beta decays and double beta decays," Phys. Rep. 797, 1-102.

Eliseev, S., K. Blaum, M. Block, C. Droese, M. Goncharov, E. Minaya Ramirez, D. A. Nesterenko, Yu. N. Novikov, and L. Schweikhard, 2013, "Phase-Imaging Ion-Cyclotron-Resonance Measurements for Short-Lived Nuclides," Phys. Rev. Lett. 110, 082501.

Eliseev, S., M. Block, A. Chaudhuri, F. Herfurth, H. J. Kluge, A. Martin, C. Rauth, and G. Vorobjev, 2007, "Octupolar excitation of ions stored in a Penning trap mass spectrometer-A study performed at SHIPTRAP," Int. J. Mass Spectrom. 262, 45-50.

Eliseev, S., D. Nesterenko, K. Blaum, M. Block, C. Droese, F. Herfurth, E. Minaya Ramirez, Yu. N. Novikov, L. Schweikhard, and K. Zuber, 2011, " $Q$ values for neutrinoless double-electron capture in ${ }^{96} \mathrm{Ru},{ }^{162} \mathrm{Er}$, and ${ }^{168} \mathrm{Yb}$," Phys. Rev. C 83, 038501.

Eliseev, S., Yu. N. Novikov, and K. Blaum, 2012, "Search for resonant enhancement of neutrinoless double-electron capture by high-precision Penning-trap mass spectrometry," J. Phys. G 39, 124003.

Eliseev, S., et al., 2011a, "Multiple-resonance phenomenon in neutrinoless double-electron capture," Phys. Rev. C 84, 012501(R).

Eliseev, S., et al., 2011b, "Resonant Enhancement of Neutrinoless Double-Electron Capture in ${ }^{152} \mathrm{Gd}$," Phys. Rev. Lett. 106, 052504.

Eliseev, S., et al., 2014, "A phase-imaging technique for cyclotronfrequency measurements," Appl. Phys. B 114, 107-128.

Eliseev, S., et al., 2015, "Direct Measurement of the Mass Difference of ${ }^{163} \mathrm{Ho}$ and ${ }^{163} \mathrm{Dy}$ Solves the $Q$-Value Puzzle for the Neutrino Mass Determination," Phys. Rev. Lett. 115, 062501.

Elliott, S. R., 2012, "Recent progress in double beta decay," Mod. Phys. Lett. A 27, 1230009.

Elliott, S. R., and M. Franz, 2015, "Colloquium: Majorana fermions in nuclear, particle, and solid-state physics," Rev. Mod. Phys. 87, 137-163.

Elliott, S. R., A. A. Hann, and M. K. Moe, 1987, "Search for double beta decay in ${ }^{100}$ Mo and ${ }^{92}$ Mo," Phys. Rev. C 36, 2129.

Elliott, S. R., et al., 2017, "Initial results from the Majorana Demonstrator," J. Phys. Conf. Ser. 888, 012035.

Engel, J., and J. Menxéndez, 2017, "Status and future of nuclear matrix elements for neutrinoless double-beta decay: A review," Rep. Prog. Phys. 80, 046301.

Faessler, A., T. Gutsche, S. Kovalenko, and F. Šimkovic, 2008, "Pion dominance in $R$-parity violating supersymmetry induced neutrinoless double beta decay," Phys. Rev. D 77, 113012.
Faessler, A., S. Kovalenko, and F. Šimkovic, 1998, "Pions in nuclei and manifestations of supersymmetry in neutrinoless double beta decay," Phys. Rev. D 58, 115004.

Fang, D. L., K. Blaum, S. Eliseev, A. Faessler, M. I. Krivoruchenko, V. Rodin, and F. Šimkovic, 2012, "Evaluation of the resonance enhancement effect in neutrinoless double-electron capture in ${ }^{152} \mathrm{Gd},{ }^{164} \mathrm{Er}$ and ${ }^{180} \mathrm{~W}$ atoms," Phys. Rev. C 85, 035503.

Fang, D. L., A. Faessler, V. Rodin, and F. Šimkovic, 2011, "Neutrinoless double- $\beta$ decay of deformed nuclei within quasiparticle random-phase approximation with a realistic interaction," Phys. Rev. C 83, 034320.

Finch, S. W., and W. Tornow, 2015, "Search for neutrinoless doubleelectron capture of ${ }^{156}$ Dy," Phys. Rev. C 92, 065503.

Fradkin, E. S., 1955, "Concerning some general relations of quantum electrodynamics, Zh. Eksp. Teor. Fiz. 29, 258-261, http://www.jetp .ac.ru/cgi-bin/dn/e_002_02_0361.pdf.

Frank-Kamenetsky, D. A., and G. V. Domogatsky, 1986, in Physics of Cosmos: Small Encyclopedia, edited by R. A. Syunyaev (Soviet Encyclopedia, Moscow).

Frekers, D., P. Puppe, J. H. Thies, P. P. Povinec, F. Šimkovic, J. Staniček, and I. Sýkora, 2011, "Double-electron capture of ${ }^{74}$ Se and the search for neutrinoless decay," Nucl. Phys. A860, 1-7.

Fremlin, J.H., and M.C. Walters, 1952, "An experimental investigation of the stability of nuclei against double beta-disintegration," Proc. Phys. Soc. London Sect. A 65, 911-915.

Fukuda, Y., et al., 1998, "Evidence for Oscillation of Atmospheric Neutrinos,” Phys. Rev. Lett. 81, 1562-1567.

Furry, W. H., 1939, "On transition probabilities in double betadisintegration," Phys. Rev. 56, 1184-1193.

Gal, A., 2000, "Limits on $n \bar{n}$ oscillations from nuclear stability," Phys. Rev. C 61, 028201.

Gando, A., et al., 2016, "Search for Majorana Neutrinos near the Inverted Mass Hierarchy Region with KamLAND-Zen," Phys. Rev. Lett. 117, 082503

Gaunt, J. A., 1929, "The triplets of helium," Phil. Trans. R. Soc. A 228, 151-196.

Gavriljuk, Ju M., V. V. Kuzminov, N. Ya. Osetrova, and S. S. Ratkevich, 2000 , "New limit on the half-life of ${ }^{78} \mathrm{Kr}$ with respect to the $2 K(2 \nu)$ capture decay mode," Phys. At. Nucl. 63, 2201-2204.

Gavriljuk, Ju. M., V. V. Kuzminov, N. Yu. Osetrova, G. V. Volchenko, and S.S. Ratkevich, 1998, "Searches for $2 K(2 \nu)$-capture mode of ${ }^{78} \mathrm{Kr}$ and ${ }^{124} \mathrm{Xe}$ decays with wall-less proportional counters," Phys. At. Nucl. 61, 1287-1292.

Gavrilyuk, Yu. M, A. M. Gangapshev, V. V. Kazalov, V. V. Kuzminov, S. I. Panasenko, S. S. Ratkevich, and S. P. Yakimenko, 2011, "Investigating $2 \mathrm{~K}$ capture in ${ }^{78} \mathrm{Kr}$ with a large-volume copper proportional counter,” Bull. Russ. Acad. Sci. Phys. 75, 526.

Gavrilyuk, Yu. M., A. M. Gangapshev, V. V. Kazalov, V. V. Kuzminov, S. I. Panasenko, and S. S. Ratkevich, 2013, "Indications of $2 \nu 2 K$ capture in ${ }^{78} \mathrm{Kr}$," Phys. Rev. C 87, 035501.

Gavrilyuk, Yu. M., A. M. Gangapshev, V. V. Kazalov, V. V. Kuzminov, S. I. Panasenko, S. S. Ratkevich, D. A. Tekueva, and S. P. Yakimenko, 2015, "A technique for searching for the $2 K$ capture in ${ }^{124} \mathrm{Xe}$ with a copper proportional counter," Phys. At. Nucl. 78, 1563-1566.

Gavrilyuk, Yu. M., A. M. Gangapshev, V. V. Kazalov, V. V. Kuzminov, S. I. Panasenko, S. S. Ratkevich, and S. P. Yakimenko, 2010, "Pulse shape analysis and identification of multipoint events in a large volume proportional counter in an experimental search for $2 K$ capture of ${ }^{78} \mathrm{Kr}$," Instrum. Exp. Tech. 53, 57-69.

Georgadze, A. S., F. A. Danevich, Yu. G. Zdesenko, V. V. Kobychev, B. N. Kropivyanskii, V. N. Kuts, A. S. Nikolaiko, and V. I. Tretyak, 1995, "Study of ${ }^{116} \mathrm{Cd}$ double beta decay with ${ }^{116} \mathrm{CdWO}_{4}$ scintillators," Phys. At. Nucl. 58, 1093-1102. 
George, S., K. Blaum, F. Herfurth, A. Herlert, M. Kretzschmar, S. Nagy, S. Schwarz, L. Schweikhard, and C. Yazidjian, 2007, "The Ramsey method in high-precision mass spectrometry with Penning traps: Experimental results," Int. J. Mass Spectrom. 264, 110-121.

George, S., et al., 2007, "Ramsey Method of Separated Oscillatory Fields for High-Precision Penning Trap Mass Spectrometry," Phys. Rev. Lett. 98, 162501.

Georgi, H. M., S. L. Glashow, and S. Nussinov, 1981, "Unconventional model of neutrino masses," Nucl. Phys. B193, 297-316.

Giuliani, A., F. A. Danevich, and V. I. Tretyak, 2018, "A multiisotope $0 \nu 2 \beta$ bolometric experiment," Eur. Phys. J. C 78, 272.

Giuliani, A., and A. Poves, 2012, "Neutrinoless double-beta decay," Adv. High Energy Phys. 2012, 857016.

Gómez-Cadenas, J. J., and J. Martín-Albo, 2015, "Phenomenology of neutrinoless double beta decay,” Proc. Sci. GSSI14 229, 004.

Goncharov, M., K. Blaum, M. Block, C. Droese, S. Eliseev, F. Herfurth, E. Minaya Ramirez, Yu. N. Novikov, L. Schweikhard, and K. Zuber, 2011, "Probing the nuclides ${ }^{102} \mathrm{Pd},{ }^{106} \mathrm{Cd}$, and ${ }^{144} \mathrm{Sm}$ for resonant neutrinoless double-electron capture," Phys. Rev. C 84, 028501.

González, M., M. Hirsch, and S. G. Kovalenko, 2016, "QCD running in neutrinoless double beta decay: Short-range mechanisms," Phys. Rev. D 93, 013017; 97, 099907(E) (2018).

Goriely, S., S. Hilaire, M. Girod, and S. Péru, 2009, "First GognyHartree-Fock-Bogoliubov Nuclear Mass Model," Phys. Rev. Lett. 102, 242501.

Graesser, M. L., 2017, "An electroweak basis for neutrinoless double $\beta$ decay,” J. High Energy Phys. 08, 99.

Graf, Lukas, Frank F. Deppisch, Francesco Iachello, and Jenni Kotila, 2018, "Short-range neutrinoless double beta decay mechanisms," Phys. Rev. D 98, 095023.

Gräff, G., H. Kalinowsky, and J. Traut, 1980, “A direct determination of the proton electron mass ratio," Z. Phys. A 297, 35-39.

Grant, I. P., 2007, Relativistic Quantum Theory of Atoms and Molecules: Theory and Computation (Springer Science+Business Media, New York).

Green, H. S., 1953, "A pre-renormalized quantum electrodynamics," Proc. Phys. Soc. London Sect. A 66, 873-880.

Hayashi, C., and Y. Munakata, 1952, "On a relativistic integral equation for bound states," Prog. Theor. Phys. 7, 481-516.

Helo, J., M. Hirsch, and T. Ota, 2016, "Long-range contributions to double beta decay revisited," J. High Energy Phys. 06, 006.

Hirsch, M., S. Kovalenko, and I. Schmidt, 2006, "Extended black box theorem for lepton number and flavor violating processes," Phys. Lett. B 642, 106-110.

Hirsch, M., K. Muto, T. Oda, and H. V. Klapdor-Kleingrothaus, 1994, "Nuclear structure calculation of $\beta^{+} \beta^{+}, \beta^{+} / \mathrm{EC}$ and EC/EC decay matrix elements," Z. Phys. A 347, 151-160.

't Hooft, Gerard, 1976, "Symmetry Breaking through Bell-Jackiw Anomalies," Phys. Rev. Lett. 37, 8-11.

Huang, K. N., M. Aoyagi, M. H. Chen, B. Crasemann, and H. Mark, 1976, "Neutral-atom electron binding energies from relaxed-orbital relativistic Hartree-Fock-Slater calculations $2 \leq Z \leq 106$," At. Data Nucl. Data Tables 18, 243.

Hyvärinen, J., and J. Suhonen, 2015, "Nuclear matrix elements for $0 \nu \beta \beta$ decays with light or heavy Majorana-neutrino exchange," Phys. Rev. C 91, 024613.

Iachello, F., and P. Van Isacker, 1991, The Interacting Boson-Fermion Model (Cambridge University Press, Cambridge, England).

Ito, Y., M. Minowa, W. Ootani, K. Nishigaki, Y. Kishimoto, T. Watanabe, and Y. Ootuka, 1997, "Development of the bolometer for the $\beta^{+} \beta^{+}$decay experiment," Nucl. Instrum. Methods Phys. Res., Sect. A 386, 439-442.
Itzykson, C., and J.-M. Zuber, 1980, Quantum Field Theory (McGraw-Hill, New York).

Ješkovský, M., D. Frekers, A. Kováčik, P. P. Povinec, P. Puppe, J. Staníček, I. Sýkora, F. Šimkovic, and J. H. Thies, 2015, “A search for double-electron capture of ${ }^{74} \mathrm{Se}$ to excited levels using coincidence/anticoincidence gamma-ray spectrometry," Nucl. Instrum. Methods Phys. Res., Sect. A 795, 268-275.

Johnson, K., and B. Zumino, 1959, "Gauge Dependence of the Wave-Function Renormalization Constant in Quantum Electrodynamics," Phys. Rev. Lett. 3, 351-352.

Kang, W. G., et al., 2013, "Ultra-low gamma-ray measurement system for neutrinoless double beta decay," Appl. Radiat. Isot. 81, 290-293.

Karpeshin, F. F., 2008, "On neutrinoless double $e$ capture problem," Phys. Part. Nucl. Lett. 5, 379-382.

Karthein, J., et al., 2019, "Direct decay-energy measurement as a route to the neutrino mass," Hyperfine Interact. 240, 61.

Ketelaer, J., et al., 2008, "TRIGA-SPEC: A setup for mass spectrometry and laser spectroscopy at the research reactor TRIGA Mainz, Nucl. Instrum. Methods Phys. Res., Sect. A 594, 162-177.

Kidd, M. F., J.H. Esterline, and W. Tornow, 2008, "Doubleelectron capture on ${ }^{112} \mathrm{Sn}$ to the excited $1871 \mathrm{keV}$ state in ${ }^{112} \mathrm{Cd}$ : A possible alternative to double- $\beta$ decay," Phys. Rev. C 78, 035504.

Kiel, H., D. Münstermann, and K. Zuber, 2003, “A search for various double beta decay modes of $\mathrm{Cd}, \mathrm{Te}$, and $\mathrm{Zn}$ isotopes," Nucl. Phys. A723, 499-514.

Kim, H. J., et al., 2007, "Searches for the $\beta^{+} / \mathrm{EC}$ decays of ${ }^{64} \mathrm{Zn}$ and ${ }^{112} \mathrm{Sn}$, and the $\beta \beta$ decay transitions of ${ }^{124} \mathrm{Sn}$ to the excited states of ${ }^{124}$ Te," Nucl. Phys. A793, 171-177.

Kluge, H. J., 2013, "Penning trap mass spectrometry of radionuclides," Int. J. Mass Spectrom. 349-350, 26-37.

Kolhinen, V. S., V. V. Elomaa, T. Eronen, J. Hakala, A. Jokinen, M. Kortelainen, J. Suhonen, and J. Äystö, 2010, "Accurate $Q$ value for the ${ }^{74}$ Se double-electron-capture decay," Phys. Lett. B 684, 17-21.

Kolhinen, V. S., T. Eronen, D. Gorelov, J. Hakala, A. Jokinen, A. Kankainen, J. Rissanen, J. Suhonen, and J. Äystö, 2011, "On the resonant neutrinoless double-electron-capture decay of ${ }^{136} \mathrm{Ce}$," Phys. Lett. B 697, 116-120.

Kolhinen, V. S., et al., 2004, "JYFLTRAP: A cylindrical Penning trap for isobaric beam purification at IGISOL," Nucl. Instrum. Methods Phys. Res., Sect. A 528, 776-787.

König, M., and G. Bollen, H. J. Kluge, T. Otto, and J. Szerypo, 1995, "Quadrupole excitation of stored ion motion at the true cyclotron frequency," Int. J. Mass Spectrom. Ion Process. 142, 95-116.

Kopeliovich, V., and I. Potashnikova, 2010, "Critical examination of the 'field-theoretical approach' to the neutron-antineutron oscillations in nuclei," Eur. Phys. J. C 69, 591-597.

Kortelainen, M., O. Civitarese, J. Suhonen, and J. Toivanen, 2007, "Short-range correlations and neutrinoless double beta decay," Phys. Lett. B 647, 128-132.

Kotila, J., J. Barea, and F. Iachello, 2014, "Neutrinoless doubleelectron capture," Phys. Rev. C 89, 064319.

Kovalenko, S., M. I. Krivoruchenko, and F. Šimkovic, 2014, "Neutrino Propagation in Nuclear Medium and Neutrinoless Double- $\beta$ Decay,” Phys. Rev. Lett. 112, 142503.

Kozlov, M. G., S. G. Porsev, M. S. Safronova, and I. I. Tupitsyn, 2015, "CI-MBPT: A package of programs for relativistic atomic calculations based on a method combining configuration interaction and many-body perturbation theory," Comput. Phys. Commun. 195, 199-213. 
Kretzschmar, M., 2007, "The Ramsey method in high-precision mass spectrometry with Penning traps: Theoretical foundations," Int. J. Mass Spectrom. 264, 122-145.

Kretzschmar, M., 2013, "Theoretical investigations of different excitation modes for Penning trap mass spectrometry," Int. J. Mass Spectrom. 349-350, 227-239.

Krivoruchenko, M. I., 1996a, "Constraint on the parity-conserving parameter of neutron-antineutron oscillations," Sov. J. Nucl. Phys. 59, 1244-1246.

Krivoruchenko, M. I., 1996b, "Suppression of neutron-antineutron oscillations in nuclei," Sov. J. Nucl. Phys. 59, 1972-1978.

Krivoruchenko, M. I., F. Šimkovic, D. Frekers, and A. Faessler, 2011, "Resonance enhancement of neutrinoless double electron capture," Nucl. Phys. A859, 140-171.

Krivoruchenko, M. I., and K.S. Tyrin, 2020, "Overlap of electron shells in $\beta$ and double- $\beta$ decays," Eur. Phys. J. A 56, 16.

Larkins, F. P., 1977, "Semiempirical Auger-electron energies for elements $10 \leq Z \leq 100$," At. Data Nucl. Data Tables 20, 311-387.

Lee, J. I., et al., 2011, "Experimental study on neutrinoless double beta decay of ${ }^{92}$ Mo," Nucl. Instrum. Methods Phys. Res., Sect. A 654, 157-161.

Lehman, L., 2014, "Extending the standard model effective field theory with the complete set of dimension-7 operators," Phys. Rev. D 90, 125023.

Lehnert, B., E. Andreotti, D. Degering, M. Hult, M. Laubenstein, T. Wester, and K. Zuber, 2016, "Double beta decays into excited states in ${ }^{110} \mathrm{Pd}$ and ${ }^{102} \mathrm{Pd}$," J. Phys. G 43, 115201.

Lehnert, B., D. Degering, A. Frotscher, T. Michel, and K. Zuber, 2016, "A search for the radiative neutrinoless double-electron capture of ${ }^{58} \mathrm{Ni}$," J. Phys. G 43, 065201.

Lehnert, B., T. Wester, D. Degering, D. Sommer, L. Wagner, and K. Zuber, 2016, "Double electron capture searches in ${ }^{74}$ Se," J. Phys. G 43, 085201.

Lehnert, B., and K. Zuber, 2011, "A first search of excited states double beta and double electron capture decays of ${ }^{110} \mathrm{Pd}$ and ${ }^{102} \mathrm{Pd}$," Phys. Lett. B 705, 47-51.

Lehnert, B., K. Zuber, E. Andreotti, and M. Hult, 2013, "New halflife limits on double- $\beta$ decays of ${ }^{110} \mathrm{Pd}$ and ${ }^{102} \mathrm{Pd}$ into excited states," Phys. Rev. C 87, 034312.

Liao, Yi, Xiao-Dong Ma, and Hao-Lin Wang, 2020, "Effective field theory approach to lepton number violating decays $K^{ \pm} \rightarrow \pi^{\mp} l^{ \pm} l^{ \pm}$: Short-distance contribution," J. High Energy Phys. 01, 127.

Lu, C. C., T. A. Carlson, F. B. Malik, T. C. Tucker, and C. W. Nestor, Jr., 1971, "Relativistic Hartree-Fock-Slater eigenvalues, radial expectation values, and potentials for atoms, $2 \leq Z \leq 126$," At. Data Nucl. Data Tables 3, 1.

Maalampi, J., and J. Suhonen, 2013, "Neutrinoless double $\beta^{+} / \mathrm{EC}$ decays," Adv. High Energy Phys. 2013, 505874.

Majorana, E., 1937, "Symmetrical theory of electrons and positrons," Nuovo Cimento 14, 171-184.

McLean, A. D., and R. S. McLean, 1981, "Roothaan-Hartree-Fock atomic wave functions Slater basis-set expansions for $Z=55-92$," At. Data Nucl. Data Tables 26, 197-381.

Mei, D.-M., I. Marshall, W.-Z. Wei, and C. Zhang, 2014, "Measuring double-electron capture with liquid xenon experiments," Phys. Rev. C 89, 014608.

Meija, J., et al., 2016, "Isotopic compositions of the elements 2013 (IUPAC technical report)," Pure Appl. Chem. 88, 293.

Meshik, A., and O. Pravdivtseva, 2017, "Weak decay of tellurium and barium isotopes in geological samples: Current status," J. Phys. Soc. Jpn. Conf. Proc. 14, 020702.
Meshik, A. P., C. M. Hohenberg, O. V. Pravdivtseva, and Ya. S. Kapusta, 2001, "Weak decay of ${ }^{130} \mathrm{Ba}$ and ${ }^{132} \mathrm{Ba}$ : Geochemical measurements," Phys. Rev. C 64, 035205.

Moshinsky, M., 1959, "Transformation brackets for harmonic oscillator functions," Nucl. Phys. 13, 104-116.

Mount, B. J., M. Redshaw, and E. G. Myers, 2010, "Double- $\beta$-decay $Q$ values of ${ }^{74} \mathrm{Se}$ and ${ }^{76} \mathrm{Ge}$," Phys. Rev. C 81, 032501(R).

Mukherjee, M., et al., 2008, "Mass measurements and evaluation around $A=22$," Eur. Phys. J. A 35, 31-37.

Myers, Ed. G., 2013, "The most precise atomic mass measurements in Penning traps," Int. J. Mass Spectrom. 349-350, 107-122.

Nakanishi, N., 1969, "A general survey of the theory of the BetheSalpeter equation,” Prog. Theor. Phys. Suppl. 43, 1-81.

Nesterenko, D. A., et al., 2012, "Double- $\beta$ transformations in isobaric triplets with mass numbers $A=124,130$, and 136," Phys. Rev. C 86, 044313.

Nesterenko, D. A., et al., 2014, "Direct determination of the atomic mass difference of ${ }^{187} \operatorname{Re}$ and ${ }^{187} \mathrm{Os}$ for neutrino physics and cosmochronology," Phys. Rev. C 90, 042501(R).

Niskanen, J., P. Norman, H. Aksela, and H. Agren, 2011, "Relativistic contributions to single and double core electron ionization energies of noble gases," J. Chem. Phys. 135, 054310.

Norman, E. B., 1985, "Improved limits on the double beta decay half-lives of ${ }^{50} \mathrm{Cr},{ }^{64} \mathrm{Zn},{ }^{92} \mathrm{Mo}$, and ${ }^{96} \mathrm{Ru}$," Phys. Rev. C 31, 1937.

Norman, E. B., and M. A. DeFaccio, 1984, "Searches for double $\beta^{+}$, $\beta^{+} /$EC and double electron-capture decays," Phys. Lett. 148B, 31.

Nozzoli, F., 2018, " ${ }^{146} \mathrm{Nd},{ }^{144} \mathrm{Sm}$ and other unexplored $2 \beta$-decay isotopes," Phys. Rev. C 97, 015501.

Otsuka, T., 1996, "Microscopic basis of the interacting boson model," Prog. Theor. Phys. Suppl. 125, 5-48.

Otsuka, T., A. Arima, and F. Iachello, 1978, "Nuclear shell model and interacting bosons," Nucl. Phys. A309, 1-33.

Päs, H., M. Hirsch, H. V. Klapdor-Kleingrothaus, and S. G. Kovalenko, 1999, "Towards a superformula for neutrinoless double beta decay," Phys. Lett. B 453, 194-198.

Päs, H., M. Hirsch, H. V. Klapdor-Kleingrothaus, and S. G. Kovalenko, 2001, "A superformula for neutrinoless double beta decay II: The short range part,” Phys. Lett. B 498, 35-39.

Päs, H., and W. Rodejohann, 2015, "Neutrinoless double beta decay," New J. Phys. 17, 115010.

Phillips II, D. G., et al., 2016, "Neutron-antineutron oscillations: Theoretical status and experimental prospects," Phys. Rep. 612, $1-45$.

Prezeau, G., M. Ramsey-Musolf, and P. Vogel, 2003, "Neutrinoless double beta decay and effective field theory," Phys. Rev. D 68 , 034016.

Pujol, M., B. Marty, P. Burnard, and P. Philippot, 2009, "Xenon in Archean barite: Weak decay of ${ }^{130} \mathrm{Ba}$, mass-dependent isotopic fractionation and implication for barite formation," Geochim. Cosmochim. Acta 73, 6834-6846.

Raduta, A. A., 2015, Nuclear Structure with Coherent States (Springer International Publishing, Cham, Switzerland).

Raduta, A. A., A. Faessler, and S. Stoica, 1991, "The $2 \nu \beta \beta$ decay rate within a boson expansion formalism," Nucl. Phys. A534, 149-172.

Raduta, A. A., A. Faessler, S. Stoica, and W. A. Kaminski, 1991, "The $2 \nu \beta \beta$ decay rate within a higher RPA approach," Phys. Lett. B 254, 7-12.

Raduta, A. A., and J. Suhonen, 1996, "Description of $\beta$ decay to excited quadrupole phonon states within a boson-expansion formalism," Phys. Rev. C 53, 176-187. 
Rahaman, S., V. V. Elomaa, T. Eronen, J. Hakala, A. Jokinen, A. Kankainen, J. Rissanen, J. Suhonen, C. Weber, and J. Äystö, 2009, "Accurate $Q$ Value for the ${ }^{112}$ Sn Double- $\beta$ Decay and Its Implication for the Search of the Neutrino Mass," Phys. Rev. Lett. 103, 042501.

Ratkevich, S. S., A. M. Gangapshev, Yu. M. Gavrilyuk, F. F. Karpeshin, V. V. Kazalov, V. V. Kuzminov, S. I. Panasenko, M. B. Trzhaskovskaya, and S. P. Yakimenko, 2017, "Comparative study of the double- $K$-shell-vacancy production in single- and doubleelectron-capture decay," Phys. Rev. C 96, 065502.

Reines, F., and C. L. Cowan, 1956, “The neutrino,” Nature (London) 178, 446-449.

Reines, F., and C.L. Cowan, 1959, "Free antineutrino absorption cross section. I. Measurement of the free antineutrino absorption cross section by protons," Phys. Rev. 113, 273-279.

Ring, P., and P. Schuck, 1980, The Nuclear Many Body Problem (Springer-Verlag, Berlin).

Rodríguez, T. R., and G. Martínez-Pinedo, 2010, "Energy Density Functional Study of Nuclear Matrix Elements for Neutrinoless $\beta \beta$ Decay," Phys. Rev. Lett. 105, 252503.

Rodríguez, T.R., and G. Martínez-Pinedo, 2012, "Calculation of nuclear matrix elements in neutrinoless double electron capture," Phys. Rev. C 85, 044310.

Rosen, S. P., and H. Primakoff, 1965, in Alpha-Beta-Gamma Ray Spectroscopy, edited by K. Siegbahn (North-Holland, Amsterdam).

Rukhadze, E., V. B. Brudanin, A. A. Klimenko, F. Piquemal, N. I. Rukhadze, Yu. A. Shitov, I. Štekl, and G. Warot, 2020, "Investigation of double beta decay of ${ }^{58} \mathrm{Ni}$ at the Modane Underground Laboratory," J. Phys. Conf. Ser. 1342, 012041.

Rukhadze, N. I., V. B. Brudanin, V. G. Egorov, A. A. Klimenko, A. Kovalik, P. Kouba, F. Piquemal, S. V. Rozov, E. Rukhadze, and A. V. Salamatin, 2016, "Search for double beta decay of ${ }^{106} \mathrm{Cd}$ in the TGV-2 experiment," J. Phys. Conf. Ser. 718, 062049.

Rukhadze, N. I., et al., 2006, "Search for double electron capture of ${ }^{106}$ Cd," Phys. At. Nucl. 69, 2117-2123.

Rukhadze, N. I., et al., 2011a, "New limits on double beta decay of ${ }^{106}$ Cd," Nucl. Phys. A852, 197-206.

Rukhadze, N. I., et al., 2011b, "Search for double beta decay of ${ }^{106}$ Cd," Bull. Russ. Acad. Sci. Phys. 75, 879.

Saakyan, R., 2013, "Two-neutrino double-beta decay,” Annu. Rev. Nucl. Part. Sci. 63, 503-529.

Saenz, C., et al., 1994, "Results of a search for double positron decay and electron-positron conversion of ${ }^{78} \mathrm{Kr}$," Phys. Rev. C 50, 1170.

Salpeter, E. E., 1952, "Mass corrections to the fine structure of hydrogen-like atoms," Phys. Rev. 87, 328-343.

Salpeter, E. E., and H. A. Bethe, 1951, "A relativistic equation for bound-state problems," Phys. Rev. 84, 1232-1242.

Sarazin, X., 2015, "Review of double beta experiments," J. Phys. Conf. Ser. 593, 012006.

Savard, G., St. Becker, G. Bollen, H. J. Kluge, R. B Moore, Th. Otto, L. Schweikhard, H. Stolzenberg, and U. Wiess, 1991, "A new cooling technique for heavy ions in a Penning trap," Phys. Lett. A 158, 247-252.

Schechter, J., and J. W. F. Valle, 1982, "Neutrinoless double- $\beta$ decay in $\mathrm{SU}(2) \times \mathrm{U}(1)$ theories," Phys. Rev. D 25, 2951.

Scielzo, N. D., et al., 2009, "Double- $\beta$-decay $Q$ values of ${ }^{130} \mathrm{Te}$, ${ }^{128} \mathrm{Te}$, and ${ }^{120} \mathrm{Te}, ”$ Phys. Rev. C 80, 025501.

Shi, W., M. Redshaw, and E. G. Myers, 2005, "Atomic masses of ${ }^{32,33} \mathrm{~S},{ }^{84,86} \mathrm{Kr}$, and ${ }^{129,132} \mathrm{Xe}$ with uncertainties $\leq 0.1 \mathrm{ppb}$," Phys. Rev. A 72, 022510.

Šimkovic, F., and M. I. Krivoruchenko, 2009, "Mixing of neutral atoms and lepton number oscillations," Phys. Part. Nucl. Lett. 6, 298-303.
Šimkovic, F., M. I. Krivoruchenko, and A. Faessler, 2011, "Neutrinoless double-beta decay and double-electron capture," Prog. Part. Nucl. Phys. 66, 446-451.

Šimkovic, F., L. Pacearescu, and A. Faessler, 2004, "Two-neutrino double beta decay of ${ }^{76} \mathrm{Ge}$ within deformed QRPA," Nucl. Phys. A733, 321-350.

Šimkovic, F., G. Pantis, J. D. Vergados, and A. Faessler, 1999, "Additional nucleon current contributions to neutrinoless double $\beta$ decay," Phys. Rev. C 60, 055502.

Smorra, C., et al., 2012, " $Q$ value and half-life of double-electron capture in ${ }^{184}$ Os," Phys. Rev. C 86, 044604.

Snijders, J. G., P. Vernooijs, and E. J. Baerends, 1981, "RoothaanHartree-Fock-Slater atomic wave functions: Single-zeta, doublezeta, and extended Slater-type basis sets for ${ }_{87} \mathrm{Fr}-{ }_{103} \mathrm{Lr}$," At. Data Nucl. Data Tables 26, 483-509.

Srinivasan, B., 1976, "Barites: Anomalous xenon from spallation and neutron-induced reactions," Earth Planet. Sci. Lett. 31, 129-141.

Sucher, J., 1980, "Foundations of the relativistic theory of manyelectron atoms," Phys. Rev. A 22, 348-362.

Suhonen, J., 1993, "Calculation of allowed and first-forbidden beta-decay transitions of odd-odd nuclei," Nucl. Phys. A563, 205-224.

Suhonen, J., 2007, From Nucleons to Nucleus: Concepts of Microscopic Nuclear Theory (Springer, Berlin).

Suhonen, J., 2011, "Neutrinoless double beta decays of ${ }^{106} \mathrm{Cd}$ revisited," Phys. Lett. B 701, 490-495.

Suhonen, J., 2012a, "Nuclear matrix elements for the resonant neutrinoless double electron capture," Eur. Phys. J. A 48, 51.

Suhonen, J., 2012b, "Theoretical investigation of the double- $\beta$ processes in ${ }^{96} \mathrm{Ru}, "$ Phys. Rev. C 86, 024301.

Suhonen, J., 2013, "Double beta decays of ${ }^{124}$ Xe investigated in the QRPA framework," J. Phys. G 40, 075102.

Suhonen, J., and O. Civitarese, 1998, "Weak-interaction and nuclearstructure aspects of nuclear double beta decay," Phys. Rep. 300, 123-214.

Sujkowski, Z., and S. Wycech, 2004, "Neutrinoless double electron capture: A tool to search for Majorana neutrinos," Phys. Rev. C 70, 052501(R).

Takahashi, Y., 1957, "On the generalized Ward identity," Nuovo Cimento 6, 371-375.

Tanabashi, M., et al. (Particle Data Group), 2018, "Review of particle physics," Phys. Rev. D 98, 030001.

ter Haar, B., and R. Malfliet, 1987, "Nucleons, mesons and deltas in nuclear matter: A relativistic Dirac-Brueckner approach," Phys. Rep. 149, 207-286.

Tretyak, V. I., F. A. Danevich, S. S. Nagorny, and Yu. G. Zdesenko, 2005 , "On the possibility to search for $2 \beta$ decay of initially unstable ( $\alpha / \beta$ radioactive) nuclei," Europhys. Lett. 69, 41-47.

Tretyak, V. I., and Yu G. Zdesenko, 2002, "Tables of double beta decay data-An update," At. Data Nucl. Data Tables 80, 83-116.

Tretyak, V. I., and Yu. G. Zdesenko, 1995, "Tables of double beta decay data," At. Data Nucl. Data Tables 61, 43-90.

Varshalovich, D. A., A. N. Moskalev, and V. K. Khersonskii, 1988, Quantum Theory of Angular Momentum (World Scientific Publishing, Singapore).

Vasil'ev, S. I., A. A Klimenko, S. B Osetrov, and A. A Pomanskii, 1993, "Experimental search for the decay of ${ }^{58} \mathrm{Ni}$ nuclei by the $e^{-} e^{+}$-conversion channel," JETP Lett. 57, 631-633, http://www .jetpletters.ac.ru/ps/1183/article_17849.shtml.

Vergados, J. D., 2011, “Transition operators entering neutrinoless double electron capture to excited nuclear states," Phys. Rev. C 84, 044328. 
Vergados, J. D., H. Ejiri, and F. Šimkovic, 2012, "Theory of neutrinoless double-beta decay," Rep. Prog. Phys. 75, 106301.

Vergados, J. D., H. Ejiri, and F. Šimkovic, 2016, "Neutrinoless double beta decay and neutrino mass," Int. J. Mod. Phys. E 25, 1630007.

Voloshin, M. B., G. V. Mitsel'makher, and R. A. Éramzhyan, 1982, "Conversion of an atomic electron into a positron and double $\beta^{+}$ decay," JETP Lett. 35, 656-659.

Wang, M., G. Audi, F. G. Kondev, W. J. Huang, S. Naimi, and Xing Xu, 2017, "The AME2016 atomic mass evaluation," Chin. Phys. C 41, 030003.

Ward, J.C., 1950, "An identity in quantum electrodynamics," Phys. Rev. 78, 182.

Weinberg, S., 1979, "Baryon- and Lepton-Nonconserving Processes," Phys. Rev. Lett. 43, 1566.

Weisskopf, V., and E. Wigner, 1930, "Over the natural line width in the radiation of the harmonius oscillator," Z. Phys. 65, 18-29.
White, G. A., 2016, A Pedagogical Introduction to Electroweak Baryogenesis (Morgan \& Claypool Publishers, San Rafael, CA).

Winter, R. G., 1955a, "Double $K$ capture and single $K$ capture with positron emission," Phys. Rev. 100, 142-144.

Winter, R. G., 1955b, "Search for double beta decay in cadmium and molybdenum," Phys. Rev. 99, 88.

XENON Collaboration, 2019, "Observation of two-neutrino double electron capture in ${ }^{124} \mathrm{Xe}$ with XENON1T," Nature (London) $\mathbf{5 6 8}$, 532-535.

Yousef, M. S., V. Rodin, A. Faessler, and F. Šimkovic, 2009, “Twoneutrino double $\beta$ decay of deformed nuclei within the quasiparticle random-phase approximation with a realistic interaction," Phys. Rev. C 79, 014314.

Zdesenko, Yu. G., and V. N. Kuts, 1986, "Experimental limits on the branching ratio of double electron capture in ${ }^{196} \mathrm{Hg}$," JETP Lett. 43, 591-595, http://www.jetpletters.ac.ru/ps/1408/article_21386.shtml. 